Florida International University FIU Digital Commons

6-24-2016

\title{
The Beam-Helicity Asymmetry for Two Pseudoscalar Mesons in Photoproduction and a Partial Wave Analysis for Excited Hyperons
}

Rafael A. Badui

Florida International University, rbadu001@fiu.edu

DOI: $10.25148 /$ etd.FIDC000736

Follow this and additional works at: https://digitalcommons.fiu.edu/etd

Part of the Nuclear Commons

\section{Recommended Citation}

Badui, Rafael A., "The Beam-Helicity Asymmetry for Two Pseudoscalar Mesons in Photoproduction and a Partial Wave Analysis for Excited Hyperons" (2016). FIU Electronic Theses and Dissertations. 2585.

https://digitalcommons.fiu.edu/etd/2585 


\section{FLORIDA INTERNATIONAL UNIVERSITY}

Miami, Florida

\section{THE BEAM-HELICITY ASYMMETRY FOR $\gamma p \rightarrow p \pi^{+} \pi^{-}$AND $\gamma p \rightarrow p K^{+} K^{-}$ AND A PARTIAL WAVE ANALYSIS FOR EXCITED HYPERONS}

A dissertation submitted in partial fulfillment of the requirements of the degree of DOCTOR OF PHILOSOPHY

in PHYSICS

by

Rafael A. Badui-Cruz 
To: Dean Michael R. Heithaus

College of Arts, Sciences and Education

This dissertation, written by Rafael A. Badui-Cruz, and entitled The Beam-Helicity Asymmetry for $\gamma p \rightarrow p \pi^{+} \pi^{-}$and $\gamma p \rightarrow p K^{+} K^{-}$and a Partial Wave Analysis for Excited Hyperons, having been approved in respect to style and intellectual content, is referred to you for judgment.

We have read this dissertation and recommend that it be approved.

Tedi Draghici

Mirroslav Yotov

Oren V. Maxwell

Brian A. Raue, Co-Major Professor

Lei Guo, Co-Major Professor

Date of Defense: June 24, 2016

The dissertation of Rafael A. Badui-Cruz is approved.

Dean Michael R. Heithaus

College of Arts, Sciences and Education

Andrés G. Gil

Vice President for Research and Economic Development

and Dean of the University Graduate School

Florida International University, 2016 


\section{DEDICATION}

This dissertation is dedicated to my parents and brother. 


\section{ACKNOWLEDGMENTS}

It is almost ten years since I first walked into FIU as a student and this journey is reaching its end. Among my professors during my freshman year were Drs. Tedi Draghici, Mirroslav Yotov, and Oren V. Maxwell, all of whom have witnessed me fail and succeed in different areas in my academic career. Each of them have had significant influence in my thought process and have always been open to discussing many topics in either mathematics or physics, for recreation or for work. I am extremely grateful to have been their student and for them to take the time to be in my dissertation committee.

My deepest gratitudes go to my advisors Drs. Brian A. Raue and Lei Guo. While research has many frustrating moments, they were always able to keep things light-hearted and fun. Their approach as my advisors were jovial and mostly hands-off, but always honest. The work environment that they developed have helped maximize the productivity of many students, including myself. I appreciate the trust that they placed in me and it has been a pleasure to be a part of their team.

I would like to thank the members of the g12 group, especially to Johann Goetz, Michael Kunkel, Carlos Salgado, and Diane Schott for their vast expertise in all g12 related aspects and the patience they had assisting me.

To my friends and colleagues Jason Bono, Adam Freese, Marianna Gabrielyan, Mahmoud Kamel, Hari Khanal, Puneet Khetarpal, Casey Neville, William Phelps, Eric Pooser, and Dipak Rimal: it has been an incredible journey. You have been amazing to work and share office spaces with. I thank you for your support, friendship, and the brazen, loud noises. Special thanks goes to Jessica Bartley for her endless support, patience, and kindness, especially throughout the writing of this document.

A mi família: muchísimas gracias por la apoya que me han dado. Yo se que aunque no pido ayuda, ustedes me ayudarían cuando sea. Lo agradezco mucho. Este documento está dedicado a ustedes. 
ABSTRACT OF THE DISSERTATION

THE BEAM-HELICITY ASYMMETRY FOR $\gamma p \rightarrow p \pi^{+} \pi^{-}$AND $\gamma p \rightarrow p K^{+} K^{-}$ AND A PARTIAL WAVE ANALYSIS FOR EXCITED HYPERONS

by

\author{
Rafael A. Badui-Cruz \\ Florida International University, 2016 \\ Miami, Florida \\ Professor Lei Guo, Co-Major Professor \\ Professor Brian A. Raue, Co-Major Professor
}

The first-time measurement of the angular dependence of the beam-helicity asymmetry for $\gamma p \rightarrow p K^{+} K^{-}$is shown and compared to $\gamma p \rightarrow p \pi^{+} \pi^{-}$. The data obtained were from the CLAS g12 experiment at Jefferson Lab. The experiment utilized a beam of circularly polarized photons with energies between 1.1 and $5.4 \mathrm{GeV}$ incident on an unpolarized liquid hydrogen target, which produced an unprecedented number of strange hadrons in photoproduction. The production mechanism for strange hadrons is not well understood. The beam-helicity asymmetry is a polarization observable that provides information on interfering production mechanisms in the reaction. It is shown that the asymmetry is sensitive to several kinematic variables that are key in modeling the reaction dynamics. Furthermore, the comparison of the beam-helicity asymmetry between the kaon and pion channels serves as a platform for the investigation of flavor dependence. A partial wave analysis on the $p K^{-}$system is also performed in a search for missing hyperon excitations. 


\section{TABLE OF CONTENTS}

CHAPTER

PAGE

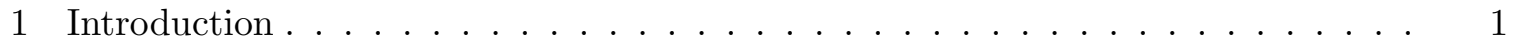

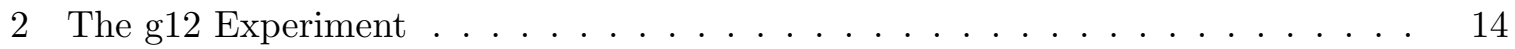

2.1 CEBAF $\ldots \ldots \ldots \ldots \ldots \ldots$

2.2 The Tagger $\ldots \ldots \ldots \ldots \ldots \ldots$

2.3 The CLAS Detector $\ldots \ldots \ldots \ldots \ldots$. . . . . . . . . . . . . . . . 17

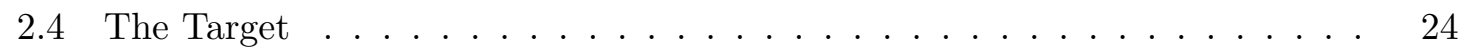

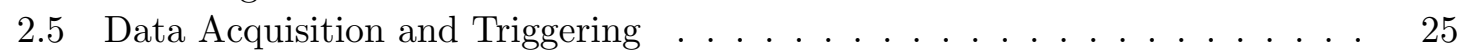

2.6 Event Reconstruction $\ldots \ldots \ldots \ldots \ldots$. . . . . . . . . . . . . . . . 27

3 Event Selection and Corrections . . . . . . . . . . . . . . . . . . . . . . . . . . . . . . .

3.1 Vertex Position and Timing . . . . . . . . . . . . . . . . . . . . . 30

3.2 Multiple Photons . . . . . . . . . . . . . . . . . . . . . . . . . . . . 34

3.3 Fiducial Region . . . . . . . . . . . . . . . . . . . . . . . . . . . . . 35

3.4 TOF Knockouts . . . . . . . . . . . . . . . . . . . . . . . . . . . . . 35

$3.5 \quad$ Energy $\operatorname{Loss} \ldots \ldots \ldots \ldots \ldots \ldots \ldots$

3.6 Beam Energy Correction . . . . . . . . . . . . . . . . . . . . . . . . . . . 38

3.7 Momentum Corrections . . . . . . . . . . . . . . . . . . . . . . . . 40

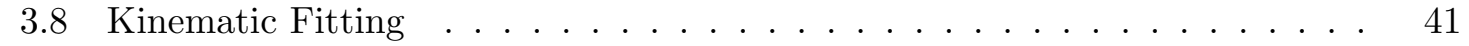

3.9 Summary . . . . . . . . . . . . . . . . . . . . . . . . . . 43

4 Beam-Helicity Asymmetry . . . . . . . . . . . . . . . . . . . . . . . . 47

4.1 Preliminaries . . . . . . . . . . . . . . . . . . . . . . 50

4.2 Beam Charge Asymmetry . . . . . . . . . . . . . . . . . . . . . . 55

4.3 Meson-Meson Plane Configuration . . . . . . . . . . . . . . . . . . 55

4.4 Neutral Baryon Configuration . . . . . . . . . . . . . . . . . . . . . 59

4.5 Positive Baryon Configuration $\ldots \ldots \ldots \ldots$

4.6 Statistical Uncertainties $\ldots \ldots \ldots \ldots$

$4.7 \quad$ Systematic Uncertainties . . . . . . . . . . . . . . . . . . . . . . . . . . . . . . . . . . . . . . . . . . . . . . . .

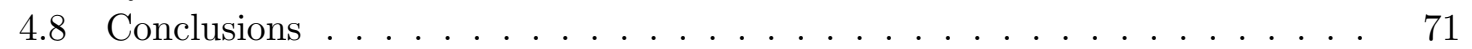

5 Partial Wave Analysis $\ldots \ldots \ldots \ldots \ldots \ldots$. . . . . . . . . . . . . . . . . . . . . . . . . .

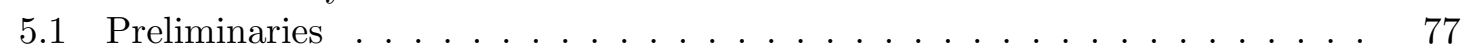

5.2 The Model $\ldots \ldots \ldots \ldots$. . . . . . . . . . . . . . . . . 80

5.3 Extended Likelihood Function $\ldots \ldots \ldots$. . . . . . . . . . . . . . 86

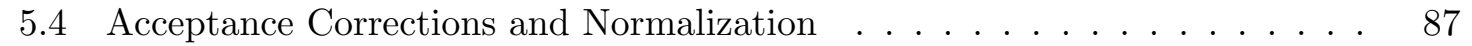

5.5 Performing the Fit . . . . . . . . . . . . . . . . . . . . . . 89

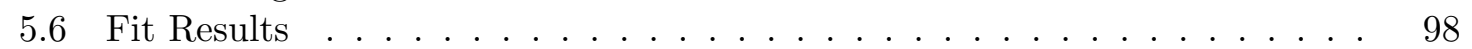

5.7 Fit Quality . . . . . . . . . . . . . . . . . . . . . . . . . . . . . . . . . . . . . . . . . . . . . . . . . . . . . . . .

5.8 Conclusions $\ldots \ldots \ldots \ldots \ldots$

6 Conclusion $\ldots \ldots \ldots \ldots \ldots \ldots \ldots$ 


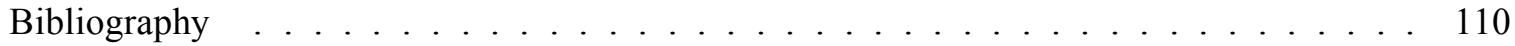

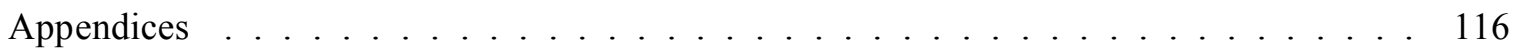

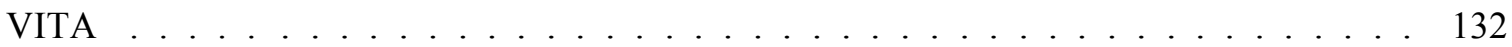




\section{LIST OF TABLES}

TABLE

PAGE

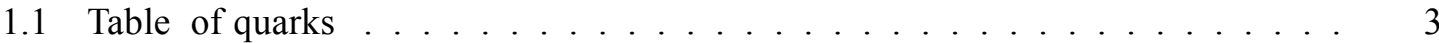

1.2 List of strange baryon states with masses greater than $2.0 \mathrm{GeV}$ as predicted in [10]. Table 1.3 shows the observed states . . . . . . . . . 7

1.3 Experimentally observed baryon resonances with $S=-1$ and masses greater than $2.0 \mathrm{GeV}$. Three- or four-star states are in bold. Table 1.2 shows the predicted $S=-1$ states from CQM ............. 8

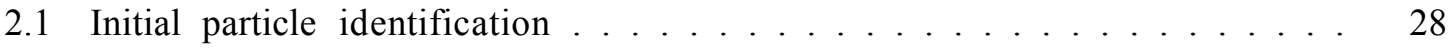

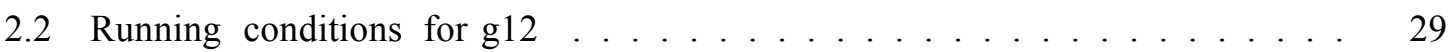

3.1 Recommended list of paddles to knockout . . . . . . . . . . . 36

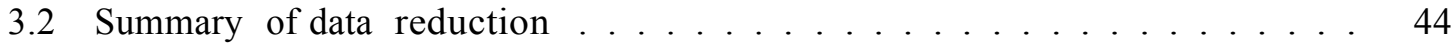

4.1 The degree of longitudinal electron polarization $\left(P_{e}\right)$ for each Møller run. The uncertainties shown are statistical uncertainties. The systematic uncertainty is estimated to be a relative $5 \% \ldots \ldots \ldots \ldots 4 \ldots \ldots \ldots$

4.2 Systematic uncertainties for pion $I^{8}$ in the meson-meson configuration . . 70

4.3 Systematic uncertainties for kaon $I^{8}$ in the meson-meson configuration . . . 70

4.4 Systematic uncertainties for kaon $I^{8}$ in the neutral baryon configuration . 71

4.5 Systematic uncertainties for kaon $I^{8}$ in the positive baryon configuration . 71

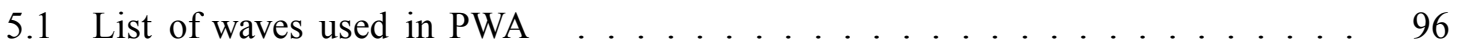

A1 Pion Data Points for Fig. $4.4 \ldots \ldots \ldots \ldots \ldots \ldots$

A2 Kaon Data Points for Fig. $4.4 \ldots \ldots \ldots \ldots \ldots$

A3 Data Points for Fig. 4.5a .................... 120

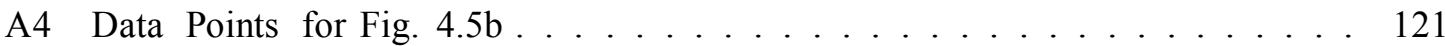


A5 Data Points for Fig. $4.6 \mathrm{a} \ldots \ldots \ldots \ldots \ldots \ldots \ldots \ldots$

A6 $\quad$ Data Points for Fig. $4.6 \mathrm{~b} . \ldots \ldots \ldots \ldots \ldots$

A7 $\quad$ Data Points for Fig. $4.6 \mathrm{c} \ldots \ldots \ldots \ldots \ldots \ldots \ldots \ldots$

A8 $\quad$ Data Points for Fig. $4.7 \mathrm{a} . \ldots \ldots \ldots \ldots$. . . . . . . . . . . . . 123

A9 Data Points for Fig. $4.7 \mathrm{~b} . \ldots \ldots \ldots \ldots$. . . . . . . . . . . . 123

A10 Data Points for Fig. $4.8 \ldots \ldots \ldots \ldots$. . . . . . . . . . . . . 124

A11 Data Points for Fig. 4.9 . . . . . . . . . . . . . . . . . . . . . . . 124

A12 Data Points for Fig. $4.11 \mathrm{a} \ldots \ldots \ldots \ldots \ldots$. . . . . . . . . . . 125

A13 Data Points for Fig. $4.11 \mathrm{~b} \ldots \ldots \ldots \ldots \ldots \ldots$

A14 Data Points for Fig. $4.11 \mathrm{c} \ldots \ldots \ldots \ldots$

A15 Data Points for Fig. $4.10 \mathrm{a} \ldots \ldots \ldots \ldots$. . . . . . . . . . . 127

A16 Data Points for Fig. $4.10 \mathrm{~b} \ldots \ldots \ldots \ldots$. . . . . . . . . . . 127

A17 Data Points for Fig. $4.12 \ldots \ldots \ldots \ldots \ldots$

A18 Data Points for Fig. 4.13 . . . . . . . . . . . . . . . . . . . . 128

A19 Data Points for Fig. $4.15 \mathrm{a} \ldots \ldots \ldots \ldots$

A20 Data Points for Fig. $4.15 \mathrm{~b}$. . . . . . . . . . . . . . . . . 129

A21 Data Points for Fig. $4.15 \mathrm{c} \ldots \ldots \ldots \ldots \ldots$

A22 Data Points for Fig. $4.14 \mathrm{a} \ldots \ldots \ldots \ldots \ldots$. . . . . . . . . . 130

A23 Data Points for Fig. $4.14 \mathrm{~b} \ldots \ldots \ldots \ldots \ldots$. . . . . . . . . . . 131 


\section{LIST OF FIGURES}

FIGURE

PAGE

1.1 Light Baryon Octet and Decuplet. The strangeness quantum number is shown on the right $\ldots \ldots \ldots \ldots \ldots$

1.2 CQM predictions for $S=0$ states matched with experimentally observed resonances. Left hand side: $N$-states. Right hand side: $\Delta$-states. Columns labeled "exp" are experimentally observed masses. Three- and four-star states are indicated by full lines, two-star states by dashed lines, one-star states by dotted lines. Columns labeled "QM" are CQM predictions. Dashed lines are unobserved states. The lines in between the columns are the states' assignment to their observed and predicted masses. The dashed lines under the "QM" columns are unobserved states. Note: assignment to CQM values are tentative. . . . . . . . . . . . . . . . . . .

1.3 Comparison of resonance widths and masses for $S=0$ (red \& magenta), $S=-1$ (blue \& cyan), and $S=-2$ (black) states with at least three-star

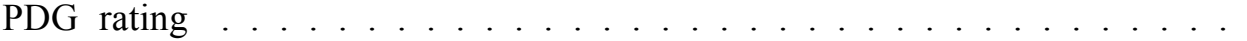

1.4 Schematic representation of the relation between experimental observables,

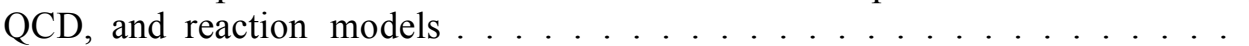

1.5 LQCD $S=-1$ predictions. Pion mass was taken to be $391 \mathrm{MeV}$. Different colors indicate different flavor representations $\left(\mathrm{SU}(3)_{F}\right)$. Blue is the flavor octet, yellow is the flavor singlet, beige is the flavor decuplet. $m_{\Omega}=1672$ $\mathrm{MeV}$ is the mass of the ground state $\Omega$. The height of the box indicates the

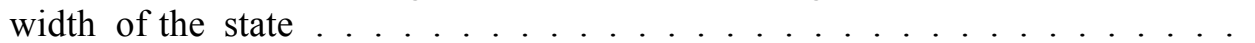

2.1 Aerial view of CEBAF prior to the construction of Hall D. Red-dashed lines

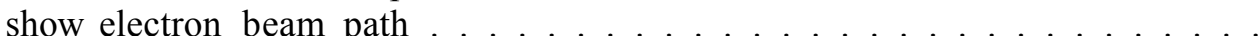

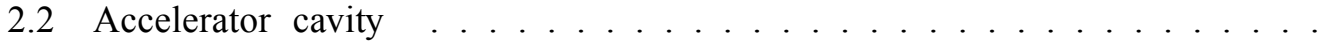


2.3 The Hall B photon tagger . . . . . . . . . . . . . . . 17

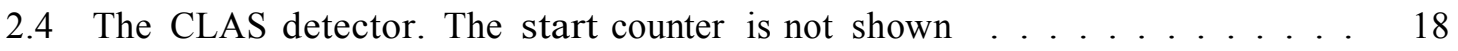

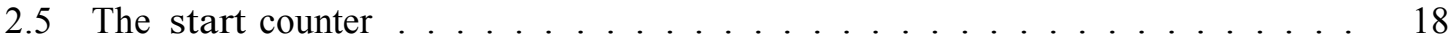

2.6 The superconducting coils . . . . . . . . . . . . . . . 19

2.7 The drift chambers system . . . . . . . . . . . . . . . 21

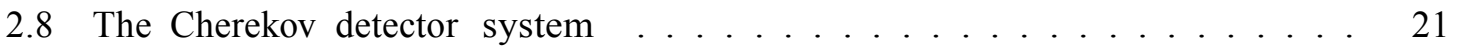

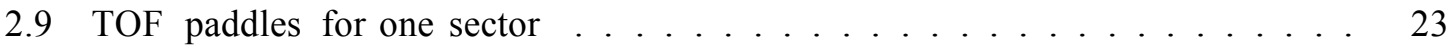

2.10 Exploded view of one of the six EC modules . . . . . . . . . . . . . 24

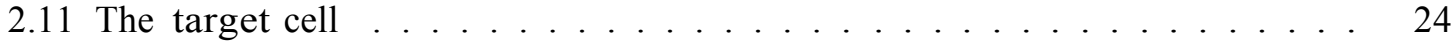

2.12 Trigger logic for one CLAS sector. The ST $\times$ TOF signal is a coincidence between any of the four start counter TDC signals with any of the 57 TOF TDC signals. ECP and ECE are the photon and electron EC thresholds, respectively. For electrons, energy must be deposited in the $\mathrm{EC}$ with coincidence with the $\mathrm{CC}(\mathrm{EC} \times \mathrm{CC})$. For photons, only within the EC $(\mathrm{ECP}) \ldots 27$

2.13 Charged particle track in DC. Particle's momentum can be determined given the chord length and sagitta . . . . . . . . . . . 28

3.1 Reconstructed vertex distributions . . . . . . . . . . . . . 31

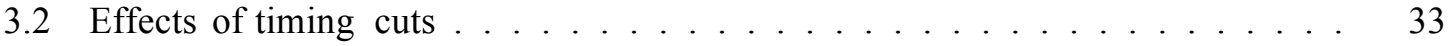

3.3 Distribution of number of tagged photons within the $2.004 \mathrm{~ns}$ window ... 34

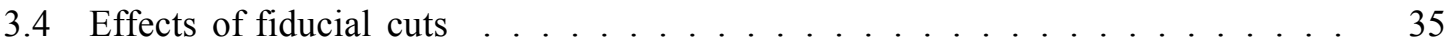

3.5 Relative occupancy of all paddles. Paddles with relative occupancy less than the dash line shown were removed from the analysis . . . . . . . . . 37

3.6 Examples of bad and good TOF paddle resolution stability . . . . . . 37

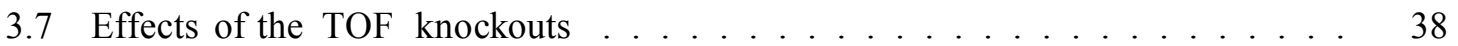

3.8 Energy loss corrections . . . . . . . . . . . . . . . . . . . . . . 39

3.9 Effects of all corrections applied on the missing mass squared . . . . . . . 40

3.10 Effects of all corrections applied on the missing momentum . . . . . . . 41

3.11 Confidence level distributions . . . . . . . . . . . . . . . . . 43 
3.12 TOF $\beta$ versus momentum after all cuts and corrections applied . . . . . 44

3.13 Missing mass consistency plots after all cuts and corrections applied ... 45

3.14 Invariant mass plots after all cuts and corrections applied . . . . . . . 46

4.1 Comparison of number of predicted and observed states as a function of resonance mass. Dotted line is the number of predicted states. Solid line is the number of observed states ................. 50

4.2 Maximon-Olsen equation. $P_{e}$ is the polarization of the incident electron beam, $P_{\gamma}$ is the polarization of the outgoing photon beam, $E_{e}$ is the energy of the incident electron beam, and $E_{\gamma}$ is the energy of the outgoing photon

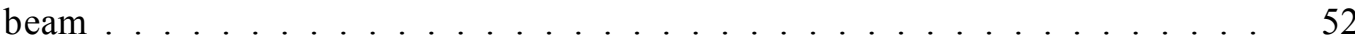

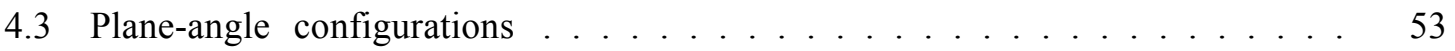

4.4 The angular dependence in the meson-meson configuration of the beamhelicity asymmetry for double-charged-pion and kaon photoproduction summed over $E_{\gamma}>1.1 \mathrm{GeV}$, momentum transfers, and invariant masses . . . . . 56

4.5 Fourier coefficients as function of center-of-mass energy in the meson-meson configuration, summed over invariant masses and momentum transfers . . .

4.6 Fourier coefficients as function of invariant masses, summed over $E_{\gamma}>1.1 \mathrm{GeV}$

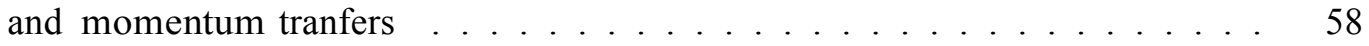

4.7 Fourier coefficients as function of momentum transfer $\ldots \ldots \ldots$

4.8 The angular dependence in the neutral baryon configuration of the beamhelicity asymmetry for double-charged-kaon photoproduction summed over $E_{\gamma}>1.1 \mathrm{GeV}, \cos \left(\theta_{\mathrm{cm}}\right)$, and invariant masses . . . . . . . . . . 60

4.9 Fourier coefficients as function of center-of-mass energy $\ldots \ldots \ldots$

4.10 Fourier coefficients as function of momentum transfer $\ldots \ldots \ldots 6$

4.11 Fourier coefficients as function of invariant masses $\ldots \ldots \ldots 62$

4.12 The angular dependence in the neutral baryon configuration of the beamhelicity asymmetry for double-charged-kaon photoproduction summed over

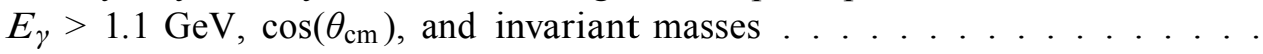


4.13 Fourier coefficients as function of center-of-mass energy . . . . . . . 63

4.14 Fourier coefficients as function of momentum transfer . . . . . . . . . 64

4.15 Fourier coefficients as function of invariant masses f . . . . . . . 65

4.16 Comparison of $c_{1}$ for the three different configurations . . . . . . . . 74

4.17 Comparison of $c_{2}$ for the three different configurations $\ldots \ldots \ldots 75$

4.18 Comparison of $c_{3}$ for the three different configurations $\ldots \ldots \ldots$

5.1 Schematic representation of the transition operator . . . . . . . . 82

5.2 Example of a gamp-formatted event. The first number at the top is the number of particles in the event. The subsequent rows contain the particle ID followed by the four-vector information of that particle . . . . . . . .

5.3 Example of a keyfile. The wave shown is for a $J=M=3 / 2 P$-wave with reflectivity $E=+1$. Note the angular momentum quantum numbers are doubled in the keyfile. . . . . . . . . . . . . . . 90

5.4 Invariant mass of $K^{+} K^{-} \ldots \ldots \ldots \ldots \ldots \ldots$

5.5 $t^{l}$ distribution with its fit. The shaded region shows which events survive the

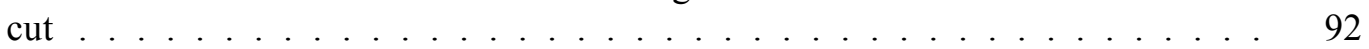

5.6 Van Hove plot for $\gamma p \rightarrow p K^{+} K^{-} \ldots \ldots \ldots \ldots$

5.7 Effects of Van Hove sector cut on $M\left(p K^{-}\right)$and $M\left(K^{+} K^{-}\right)$. Plots are normalized so that the sum over all bins is $1 \ldots \ldots 94$

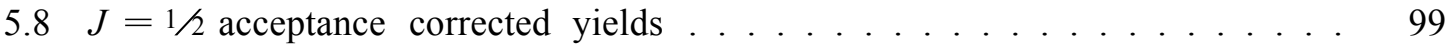

$5.9 J=3 / 2 P$ waves acceptance corrected yields . . . . . . . . . . 100

$5.10 \mathrm{~J}=3 / 2 \mathrm{D}$ waves acceptance corrected yields . . . . . . . . . . 101

$5.11 J=5 / 2 D$ waves acceptance corrected yields . . . . . . . . . . . 102

$5.12 N$ acceptance corrected yield . . . . . . . . . . . . . 103

5.13 Background wave acceptance corrected yield . . . . . . . . . . . 103

5.14 Comparison between PWA predicted distributions and data in the $1.47<M$ $\left(p K^{-}\right)<1.6 \mathrm{GeV}$ mass region . . . . . . . . . . . . . . 105 


\section{CHAPTER 1}

\section{Introduction}

It has become common knowledge that atoms are the basic building blocks of matter. Atoms are composed of a positively charged nucleus with electrons orbiting it. The nucleus itself is made up of protons and neutrons, which are then used to classify atoms by element and isotope. An element is distinguished by the number of protons inside the nucleus and an isotope by the number of neutrons. The hydrogen atom is the simplest element consisting of one proton in its nucleus. If the electromagnetic force were the only force present within the nucleus and since two like charges repel, it would be impossible for a nucleus consisting of two or more protons to exist. Consequently, elements beyond hydrogen would not be able to exist. However, the most abundant helium atom contains two protons in its stable nucleus along with two neutrons. It can be deduced that there is another force interacting within the nucleus that keeps the nucleus together and involves the neutrons. These interactions between the protons and neutrons must overcome the electric repulsion at short distances and, as this force is not observed at larger distances, its strength must weaken at large distances. This force is known as the strong force.

Shortly after the discovery of the neutron, Heisenberg provided remarkable insight into the strong force by postulating that since protons and neutrons have almost identical masses, they are really two flavors of the same particle called the nucleon, and that there must be some process that transforms one into the other 11. Mathematically, protons and neutrons are the basis states for the two-dimensional irreducible representation of $\mathrm{SU}(2)$ known as isospin $1 / 2$. The process that transforms one into the other was discovered to be the exchange of pions. The picture that Heisenberg provided, however, was incomplete.

Several particles, more massive than protons and neutrons, but always decaying into either a proton or neutron, were later discovered. Some of the particles decayed in $\sim 10^{-23}$ seconds and were considered to be excited nucleon states. In contrast, a massive particle that decayed into a nucleon and a pion in $\sim 10^{-10}$ seconds was also discovered. This particle was called the $\Lambda$. In addition to its peculiar lifetime, the $\Lambda$ was produced 
copiously and always in association with a kaon, a particle resembling a more massive pion. This observation led to Pais's theory of associated production [2]. Pais explained that particles that are produced in pairs (associated production) are produced through a different process than their decays. In the case of the $\Lambda$, it was produced through the strong interaction and decayed through the weak interaction. This phenomenon was considered to be strange, which led to the eventual name of the quantum number and quark flavor. While Heisenberg's theory was incomplete, it established an important role of symmetry and representation theory in particle physics.

Following Heisenberg and Pais, Gell-Mann and Zweig postulated the existence of quarks coming in three flavor:17, with exchanges in color being mediated by gluons [3]. An important observation (or lack of) is that individual quarks have not been observed. This led to an important property of the interactions between quarks known as confinement. Confinement means a lone quark will not be observed as the force between two quarks will get stronger as they get further apart. When the two quarks are pulled sufficiently far apart, the large binding energy will be sufficient for a quark-antiquark pair to form from the vacuum and bind with the original two in a process called hadronization. With the discovery of the $\Delta^{++}$, a particle composed of three identical up-flavored quarks, the color charge of quarks was discovered. The color charge of a quark is necessary to reconcile the fact that three identical fermions are in a completely symmetric state, which is forbidden by Fermi statistics. Along with color, confinement implies that all observed states must be invariant under a $S U(3)$ action. Ceding to the typical analogy in the theory of visual colors, labeling the three color charges red, green, blue, their anticolors cyan (antired), magenta (antigreen), yellow (antiblue), confinement amounts to a bound state being colorless (white or black). Particles composed of quarks and gluons in a bound state are known as hadrons. Baryons are a subcategory of hadrons that are composed of three quarks in a red-bluegreen color combination (white states) while mesons are hadrons composed of a quark and an antiquark pair in a color-anticolor combination (black states). The theory consequently developed is known as quantum chromodynamics (QCD).

\footnotetext{
${ }^{1}$ Currently, six flavors of quarks are known and are split into three generations. $c f$. Table 1.1
} 
The three flavors of quarks generalized the concept of isospin, which is carried by the up- and down-flavored quarks. With the third quark, Gell-Mann and Zweig, along with Nakano and Nishijima [4], postulated the existence of a new quantum number, known as strangeness (denoted by $S$ ), and its relationship with the charge, isospin, and baryon number. The $\Lambda$ was classified as a baryon with strangeness $S=-1$. Using this model, Gell-Mann was able to categorize the known particles of his time, consolidate competing theories, and predict the $\Omega^{-}$baryon, which was observed several years after his prediction. Table 1.1 shows a table of the known quarks. The up, down, and strange quarks are considered to be light quarks while the other three are heavy. Figure 1.1 shows the light baryon octet and decuplet organized using Gell-Mann's formalism. Quantum chromodynamics is the fundamental theory of the strong interactions. Along with confinement it exhibits another unique property known as asymptotic freedom. Contrary to confinement, asymptotic freedom means that at high energies, quarks and gluons are weakly interacting. Asymptotic freedom was theorized by Wilczek, Gross, and Politzer [5, 6]. Unlike asymptotic freedom, it is currently unknown how to prove confinement within the framework of QCD, which makes the connection between QCD and nuclear physics a murky area, yet abundant with discovery opportunities.

\begin{tabular}{|c|c|c|c|c|c|}
\hline Quark & Charge & Strangeness & Charmness & Bottomness & Topness \\
\hline up & $2 / 3$ & 0 & 0 & 0 & 0 \\
down & $-1 / 3$ & 0 & 0 & 0 & 0 \\
\hline charm & $2 / 3$ & 0 & 1 & 0 & 0 \\
strange & $-1 / 3$ & -1 & 0 & 0 & 0 \\
\hline top & $2 / 3$ & 0 & 0 & 0 & 1 \\
bottom & $-1 / 3$ & 0 & 0 & -1 & 0 \\
\hline
\end{tabular}

Table 1.1: Table of quarks. Source: 7] 


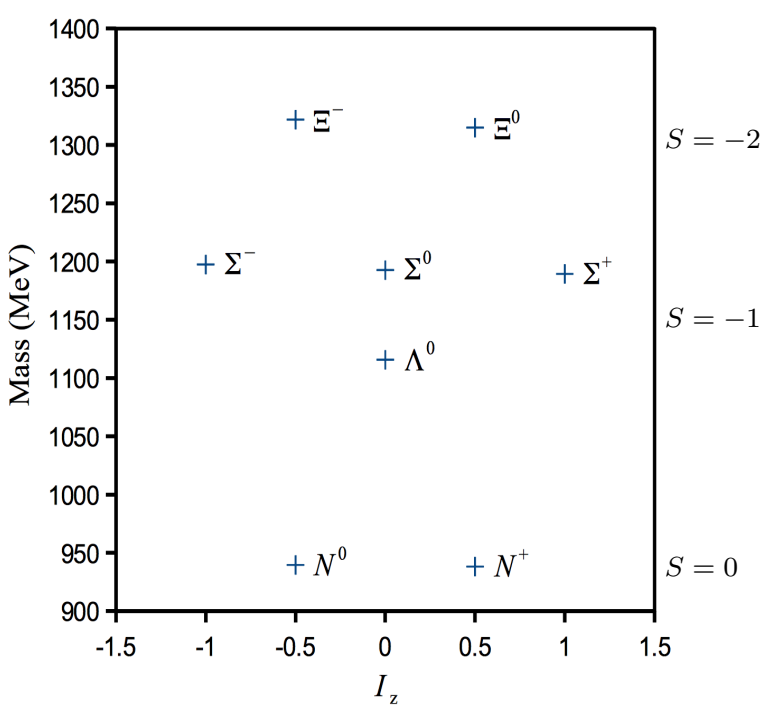

(a) Light Baryon Octet

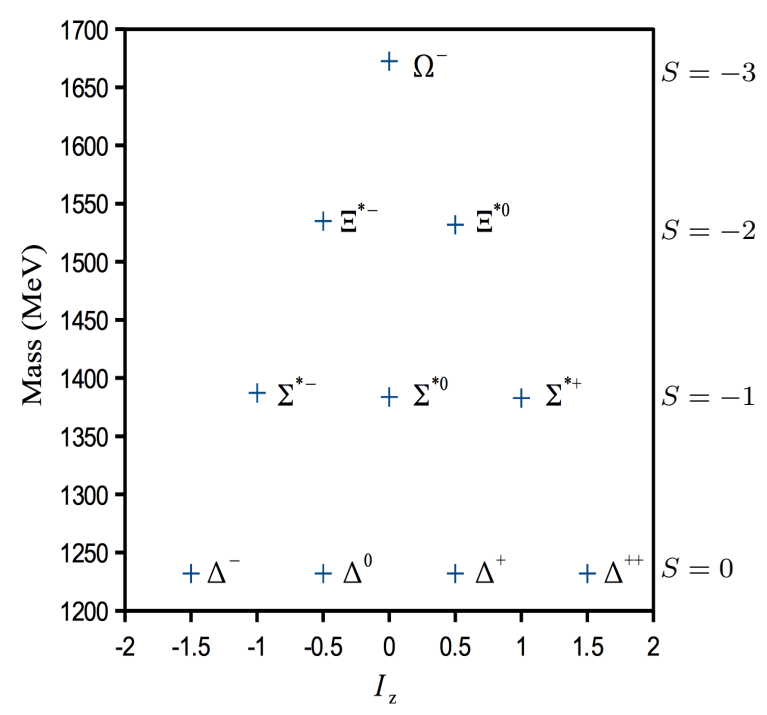

(b) Light Baryon Decuplet

Figure 1.1: Light Baryon Octet and Decuplet. The strangeness quantum number is shown on the right. Source: 8

One of the main difficulties of QCD is that it is a non-abelian gauge theory, with symmetry group $\mathrm{SU}(3)$, which makes the theory difficult to solve analytically. Consequently, many approximation schemes have been developed to aid our understanding at different energy regimes. Perturbative QCD takes advantage of the asymptotic freedom of quarks at high energies and expands on the small coupling constant in this regime. While this approach has been highly successful at high energies where the quarks and gluons can be taken to be approximately free, it is of no use at lower energies where confinement causes quarks to hadronize.

Another way of studying QCD is through effective field theories. Effective field theories consider only the main contributions for a given energy range while suppressing higher and lower energy effects, which makes calculations at the relevant energies easier. One of the most successful models is the constituent quark model (CQM). The CQM is an effective field theory that considers only the valence quark degrees of freedom inside a hadron. These quarks attain an effective mass and nonpointlike structure. Although the effective masses 
of the quarks may differ greatly from their true values, the CQM makes specific predictions on hadron excitations and their masses as shown in Fig. 1.2 and Table 1.2

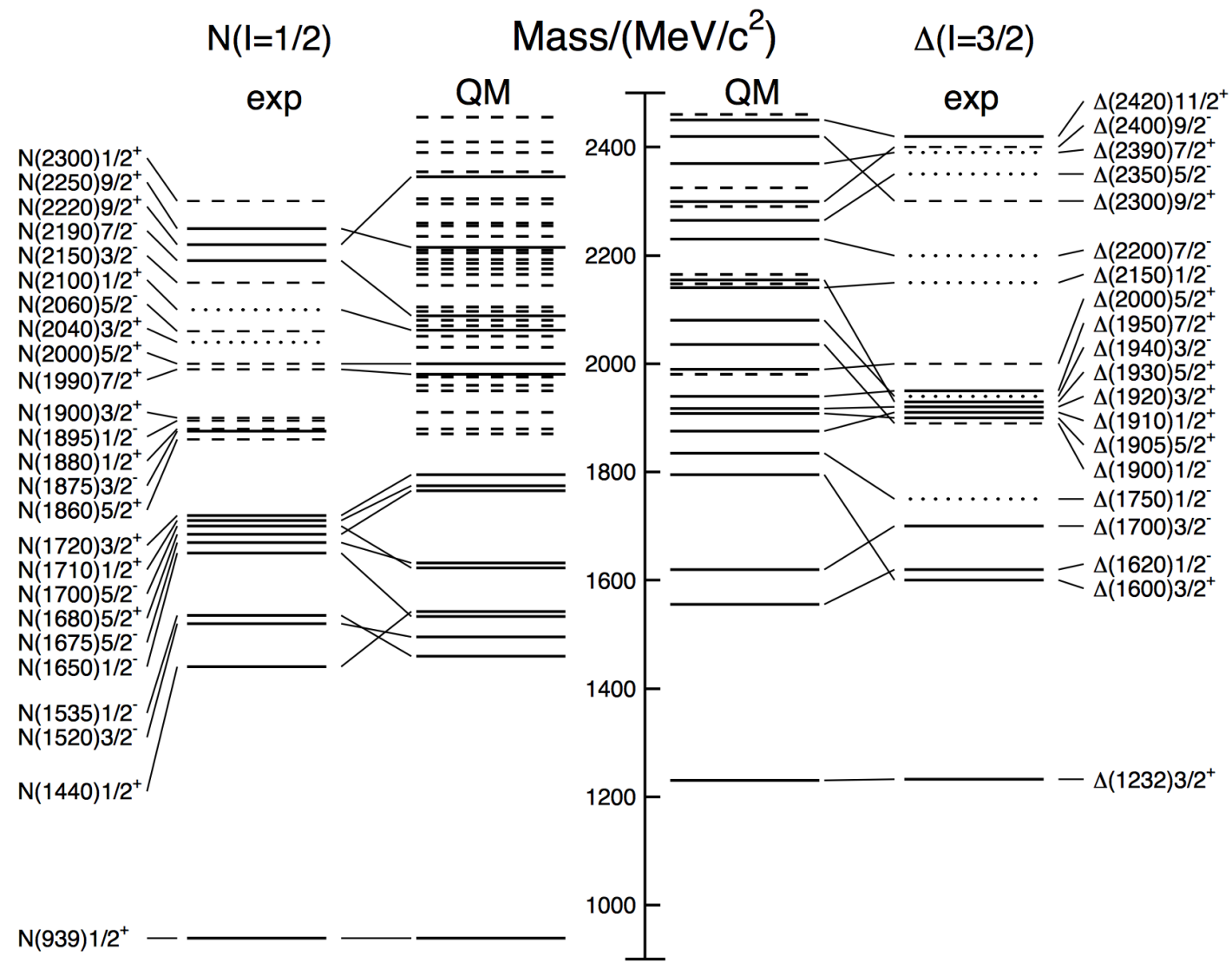

Figure 1.2: CQM predictions for $S=0$ states matched with experimentally observed resonances. Left hand side: $N$-states. Right hand side: $\Delta$-states. Columns labeled "exp" are experimentally observed masses. Three- and four-star states are indicated by full lines, two-star states by dashed lines, one-star states by dotted lines. Columns labeled "QM" are CQM predictions. Dashed lines are unobserved states. The lines in between the columns are the states' assignment to their observed and predicted masses. The dashed lines under the "QM" columns are unobserved states. Note: assignment to CQM values are tentative. Source: 7

As demonstrated by Fig. 1.2 , there are a number of nucleon states that are predicted by the CQM that have not been observed, a problem commonly known as the missing baryon

\footnotetext{
${ }^{2}$ The Particle Data Group (PDG) is an international collaboration that compiles and evaluates measurements related to particle physics and related areas. The PDG classifies baryon resonances according to a star rating based on existing evidence. The ratings range from one star to four stars. Four stars: existence is certain. Three stars: existence is likely to certain, but further confirmation is desirable. Two stars: evidence of existence is only fair. One star: evidence of existence is poor 7 .
} 
problem. Reference [9] proposes that discovering unobserved $S=0$ baryons by analyzing the $S=-1$ sector is possible as some excited nucleons have strong decay modes into $\Lambda$ s and $\Sigma$ s. Comparing Tables 1.2 and 1.3 , there are many unobserved but predicted baryons with $S=-1$ and masses greater than $2.0 \mathrm{GeV}$ as well. As shown in Fig. 1.3, $S=-1$ and $S=-2$ states are narrower than $S=0$ states. This potentially makes the experimental discovery of these states easier. As producing baryons is more difficult the more strangeness it contains and widths get larger as the mass increases, searching for $S=-1$ states is a good compromise for experiments: $S=-1$ states are narrower than the $S=0$ ones and yield more statistics than the $S=-2$ states.

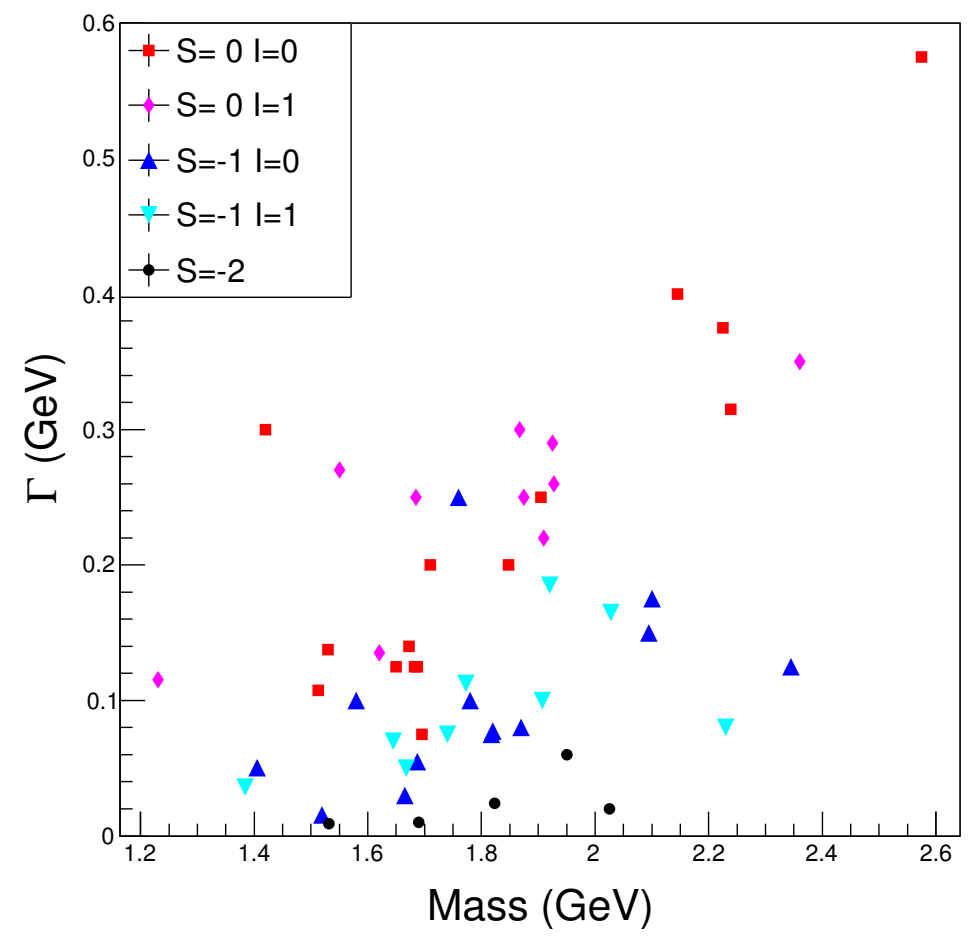

Figure 1.3: Comparison of resonance widths and masses for $S=0$ (red \& magenta), $S=-1$ (blue \& cyan), and $S=-2$ (black) states with at least three-star PDG rating.

These resonances could be missing because either they have not yet been discovered or do not exist. A possible reason for the excess states according to the CQM is that there are too many degrees of freedom. An alternative model to the CQM with fewer degrees of 


\begin{tabular}{|c|c|c|}
\hline State & $J^{P}$ & Predicted Mass $(\mathrm{MeV})$ \\
\hline$\Lambda$ & $\frac{1}{2}^{-}$ & $2015,2095,2160,2195,2235,2280$ \\
\hline$\Sigma$ & $\frac{1}{2}^{-}$ & $2110,2155,2165,2205,2260,2275$ \\
\hline$\Lambda$ & $\frac{3}{2}^{-}$ & $2030,2110,2185,2230,2290$ \\
\hline$\Sigma$ & $\frac{3}{2}^{-}$ & $2120,2185,2200,2215,2265,2290$ \\
\hline$\Lambda$ & $\frac{5}{2}^{-}$ & $2180,2225,2240,2295$ \\
\hline$\Sigma$ & $\frac{5}{2}^{-}$ & $2205,2250,2270,2280$ \\
\hline$\Lambda$ & $\frac{7}{2}^{-}$ & 2150,2230 \\
\hline$\Sigma$ & $\frac{7}{2}^{-}$ & 2245 \\
\hline$\Lambda$ & $\frac{1}{2}^{+}$ & $2010,2105,2120,2195,2270$ \\
\hline$\Sigma$ & $\frac{1}{2}^{+}$ & $2005,2030,2105,2240$ \\
\hline$\Lambda$ & $\frac{3}{2}^{+}$ & $2050,2080,2120,2160$ \\
\hline$\Sigma$ & $\frac{3}{2}^{+}$ & $2010,2030,2045,2085,2115,2155$ \\
\hline$\Lambda$ & $\frac{5}{2}^{+}$ & $2035,2115,2180$ \\
\hline$\Sigma$ & $\frac{5}{2}^{+}$ & $2030,2095,2110,2130$ \\
\hline$\Lambda$ & $\frac{7}{2}^{+}$ & 2120 \\
\hline$\Sigma$ & $\frac{7}{2}^{+}$ & 2060,2125 \\
\hline
\end{tabular}

Table 1.2: List of strange baryon states with masses greater than $2.0 \mathrm{GeV}$ as predicted in [10]. Table 1.3 shows the observed states. 


\begin{tabular}{|c|c|c|c|c|c|}
\hline$\Lambda$ States & $J^{P}$ & Star Rating & $\Sigma$ States & $J^{P}$ & Star Rating \\
\hline$\Lambda(2000)$ & & $*$ & $\Sigma(2000)$ & $1 / 2^{-}$ & $*$ \\
\hline$\Lambda(2020)$ & $7 / 2^{+}$ & $*$ & $\Sigma(2030)$ & $7 / 2^{+}$ & $* * * *$ \\
\hline$\Lambda(2050)$ & $3 / 2^{-}$ & $*$ & $\Sigma(2070)$ & $5 / 2^{+}$ & $*$ \\
\hline$\Lambda(2100)$ & $7 / 2^{-}$ & $* * * *$ & $\Sigma(2080)$ & $3 / 2^{+}$ & $* *$ \\
\hline$\Lambda(2110)$ & $5 / 2^{+}$ & $* * *$ & $\Sigma(2100)$ & $7 / 2^{-}$ & $*$ \\
\hline$\Lambda(2325)$ & $3 / 2^{-}$ & $*$ & $\Sigma(2250)$ & & $* * *$ \\
\hline$\Lambda(2350)$ & $9 / 2^{+}$ & $* * *$ & $\Sigma(2455)$ & & $* *$ \\
\hline \multirow[t]{3}{*}{$\Lambda(2585)$} & & $* *$ & $\Sigma(2620)$ & & $* *$ \\
\hline & & & $\Sigma(3000)$ & & $*$ \\
\hline & & & $\Sigma(3170)$ & & $*$ \\
\hline
\end{tabular}

Table 1.3: Experimentally observed baryon resonances with $S=-1$ and masses greater than $2.0 \mathrm{GeV}$. Three- or four-star states are in bold. Table 1.2 shows the predicted $S=-1$ states from CQM. Source: 7 
freedom is the diquark model [11]. Because of the reduced degrees of freedom, fewer states are allowed. Like the CQM, the diquark model also treats a baryon as having three valence quarks, but only two of them are strongly coupled. References 12 14 make predictions of the excitation spectra for $S=0$ and $S=-1$ states with masses less than $2.0 \mathrm{GeV}$. Compared to experimental values, their predictions are accurate but, like the CQM, also have missing states, albeit fewer.

While effective field theories have been highly successful at explaining hadronic phenomena, they have an inherent difficulty as demonstrated by the CQM and diquark models: the identification of the relevant and irrelevant degrees of freedom as the number of excited states predicted follow from the number of effective degrees of freedom. As such, the comparison of the experimentally determined excitation spectrum to model predictions provides constraints on what the relevant degrees of freedom are, which is crucial to the understanding of QCD 11]. Figure 1.4 contains a schematic showing the cooperation between theory models and experimental observables.

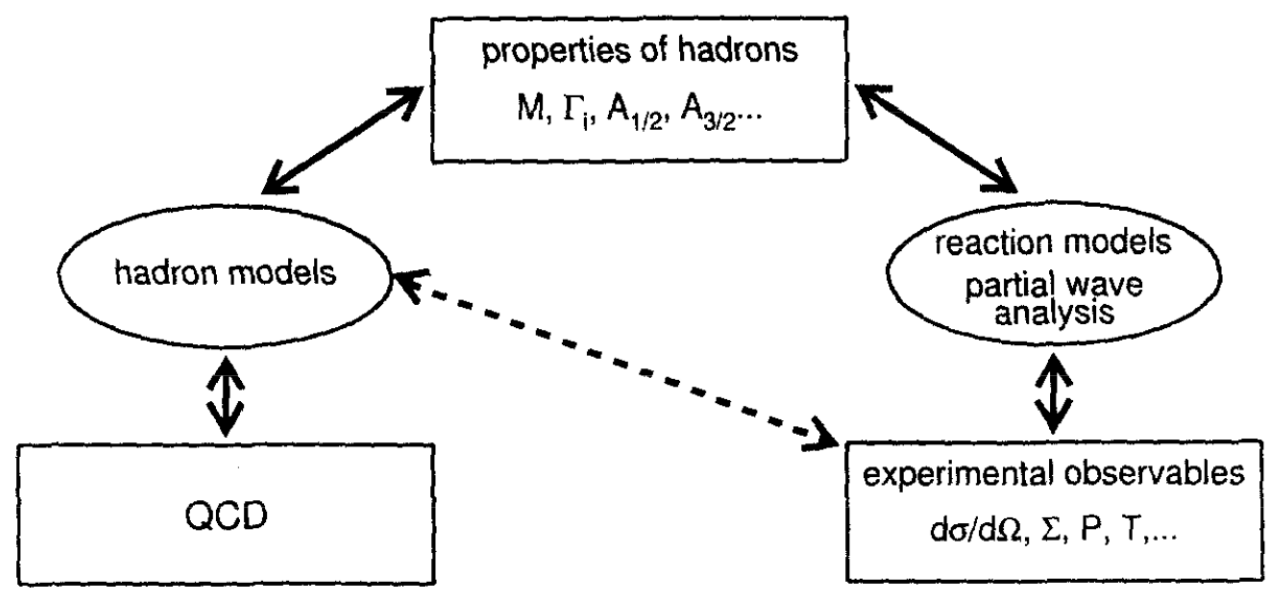

Figure 1.4: Schematic representation of the relation between experimental observables, QCD, and reaction models. Source: 11 .

Another way of approaching QCD is through Lattice QCD (LQCD). Lattice QCD is a nonperturbative, first-principles approach that approximates space-time as a lattice. The continuum limit is then observed as the lattice spacing goes to zero; it is a way of 
regularizing QCD. Lattice QCD also makes baryon excitation calculations, shown in Fig. 1.5 [15. Even with this alternative approach, the missing baryon problem still prevails as there are an excess number of predicted states. Exacerbating the problem, Fig. 1.5 shows overlap between the states, which makes it difficult to isolate them. Furthermore, by approximating physics on a lattice, Poincaré symmetry $\mathrm{b}^{3}$ is lost and cannot be recovered in a straightforward way, even in the continuum limit. Conservation of angular momentum is lost as a result. Even with these drawbacks, LQCD remains as a leading theoretical tool to study QCD from first principles at energies where confinement dominates.

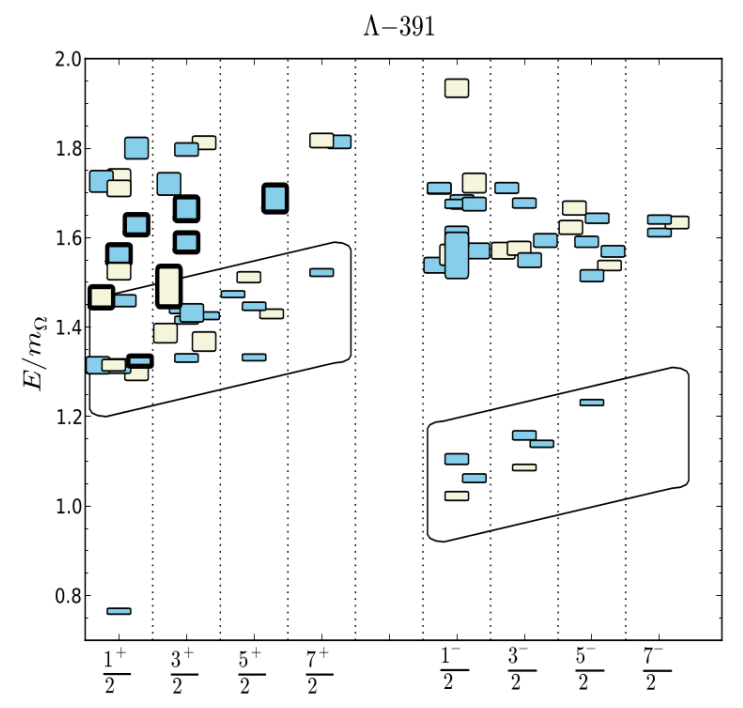

(a) LQCD $\Lambda$ predictions

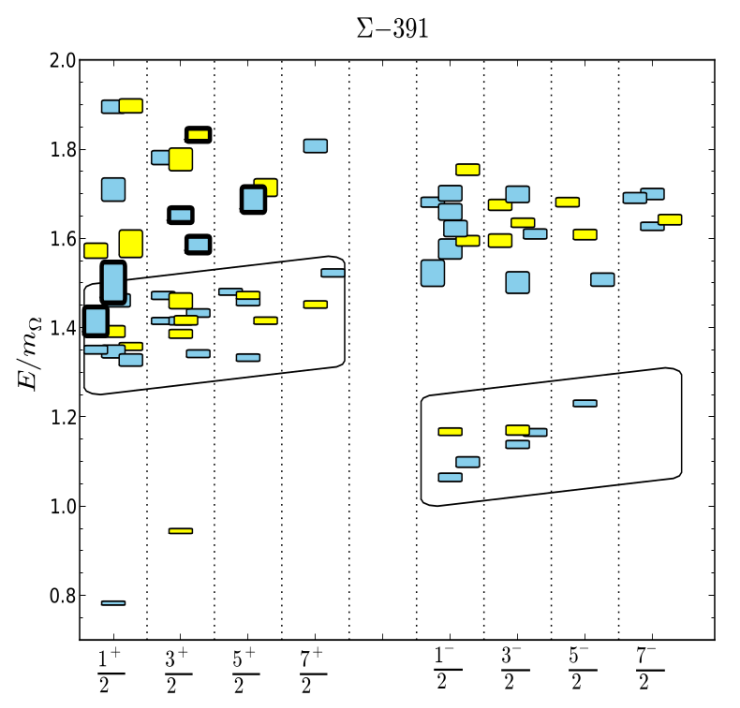

(b) LQCD $\Sigma$ predictions

Figure 1.5: LQCD $S=-1$ predictions. Pion mass was taken to be $391 \mathrm{MeV}$. Different colors indicate different flavor representations $\left(\mathrm{SU}(3)_{F}\right)$. Blue is the flavor octet, yellow is the flavor singlet, beige is the flavor decuplet. $m_{\Omega}=1672 \mathrm{MeV}$ is the mass of the ground state $\Omega$. The height of the box indicates the width of the state. Source: 15]

The primary experimental technique used to identify states is through spectroscopy. Spectroscopy is the study of the interactions between matter and radiation. Originally, spectroscopy was used to determine the structure of atoms. In an atomic spectroscopy experiment, the electrons of an atom are excited to higher energy states by absorption of a photon. When the electron transitions to a lower energy state, it emits a photon with energy

\footnotetext{
${ }^{3}$ The group of Minkowski isometries, $\mathbb{R}^{1,3} \rtimes \mathrm{SO}(1,3)$, composed of translations, rotations, and boosts.
} 
equal to the amount the electron lost. The photon's energy is uniquely determined from its wavelength. The wavelength is recorded and the experiment is repeated. The results of atomic spectroscopy led to the development of quantum electrodynamics (QED) [16], one of the most successful theories made. With the advances in engineering and low-temperature physics came the ability to probe deeper within the atom, into the nucleus, and eventually into nucleons by utilizing higher energy probes. With higher energies, particles are more abundant and diverse, usually leading to surprising phenomena not observed previously, e.g. the $\Lambda$ hyperon discovery. Similar to atomic spectroscopy, hadron spectroscopy excites the nucleon to understand its structure. The radiation emitted from these excited particles is typically the emission of mesons.

While high energy probes can be obtained naturally by cosmic rays, they are uncontrollable. Instead, they are produced at accelerator facilities, which allow for control of the energies produced and at a higher rate of production. The data analyzed in this work were obtained from the g12 experiment using the CEBAF Large Acceptance Spectrometer (CLAS) at the Thomas Jefferson National Accelerator Facility (Jefferson Lab). A notable feature of the CLAS detector is its large acceptance optimized for the simultaneous detection of multiple particles. It is roughly spherical, surrounding the target, and can measure the momenta and angles of the particles produced with almost $4 \pi$ sr coverage. The g12 experiment utilized a circularly polarized photon beam incident on a liquid hydrogen target (Chapter 2) and produced an unprecedented number of strange-particle final states, which makes it favorable for the study of hyperon resonances. Excited hyperon states typically decay to a proton and a negative kaon and are produced in association with a positive kaon. The reaction studied to investigate these states was $\gamma p \rightarrow p K^{+} K^{-}$following the reaction chain of producing an excited hyperon $Y^{*}$ through $\gamma p \rightarrow Y^{*} K^{+} \rightarrow p K^{+} K^{-}$.

The lifetimes of these excited states are brief $\left(\sim 10^{-24} \mathrm{~s}\right)$, yielding widths of several hundred $\mathrm{MeV}$, which overlap one another in the mass spectrum. Isolating them is a difficult task by simply "bump hunting" or using cross section measurements alone. Extracting properties, such as their spin and parity quantum numbers, is even more difficult

\footnotetext{
${ }^{4}$ Continuous Electron Beam Accelerator Facility
} 
given cross sections and angular distributions alone. Furthermore, the background process, $\gamma p \rightarrow p X \rightarrow p K^{+} K^{-}$, producing an intermediate meson yields the same final state and contaminates the hyperon signal. Polarization observables, however, are sensitive to the interference of competing hadronic processes and will aid in probing the production mechanisms of these resonances. The modeling of the photoproduction of two pseudoscalar mesons involves eight independent complex amplitudes, meaning there are 16 independent quantities. Cross section measurements only constrain the sum of the squares of these amplitudes 17]. Polarization observables are necessary to constrain the other variables.

The polarization observable measured in this work is the beam-helicity asymmetry, $I^{\odot}$. This observable, emerging from the beam's circular polarization, can serve as one of the independent quantities in the reaction model. Its sensitivity to key kinematic variables is also investigated (Chapter 4 ). $I^{\odot}$ is also measured for $\gamma p \rightarrow p \pi^{+} \pi^{-}$to investigate its flavor dependence. One of the key differences between $\gamma p \rightarrow p K^{+} K^{-}$and $\gamma p \rightarrow p \pi^{+} \pi^{-}$is the well-established $\Delta^{++}$resonance coming from $p \pi^{+}$. The strange counterpart, $p K^{+}$, would be an exotic baryon made of five valence quarks. Searches for this exotic state have concluded that it does not exist [18]. Because of this, it is expected that $I^{\odot}$ be different for both reactions and its modeling easier for $\gamma p \rightarrow p K^{+} K^{-}$.

While polarization observables are essential to a complete reaction model, ultimately the goal of interest is in extracting unobserved resonances, if any. To identify these resonances, their spin, mass, and width must be measured. Due to the overlapping nature of the resonances, a partial wave analysis (PWA) can be applied following the formalism in references 19 21. Concisely, a PWA models the reactions with linear combinations of orthogonal states, each with a certain quantum number that yield an expected angular intensity. That model is fit to the data and the relative contribution for each wave given in the model is obtained.

In a scattering experiment, the operator that relates the collision between the reaction inducers (denoted $\mid$ in $\rangle$ ) and the asymptotic outgoing states (denoted $\mid$ out $\rangle$ ) is the $S$-matrix. The $S$-matrix models the interactions of the incoming states that produce the out states. The elements of the $S$-matrix are called the scattering amplitudes. The physical constraints 
of causality, crossing symmetry, and unitarity translate to constraints on the $S$-matrix elements. Briefly, causality requires that the scattering amplitudes be analytic functions of the center-of-mass energy $(W)$ and momentum transfers $\left(t_{\gamma \rightarrow p_{i}}\right.$ where $p$ is a final-state product of the reaction, $i$ ranges from 1 to $N-1$, and $N$ is the number of final state particles). Poles in the amplitudes as a function of the energy signify the existence of a resonance. A cut along the real part of $W$ corresponds to the energies for which the process is allowed. References 22,23 contain detailed information on properties of the $S$-matrix and their consequences. Given a reaction model, the amplitudes can be obtained from specifying the angular distributions of the outgoing states. In this work, a PWA is applied to the $p K^{-}$mass spectrum for the reaction $\gamma p \rightarrow p K^{+} K^{-}$. For this particular case, the angular distribution of the final state proton in the rest frame of the $p K^{-}$system is measured and the scattering amplitudes are obtained by fitting to the reaction model described in Chapter 5 . 


\section{CHAPTER 2}

\section{The g12 Experiment}

The experiment from which this work obtained its data is known as g12 and was conducted at Jefferson Lab, located in Newport News, VA. Jefferson Lab currently houses four experimental halls (labeled A, B, C, and D) and the Continuous Electron Beam Accelerator Facility (CEBAF) (Section 2.1). The g12 experiment was a photoproduction experiment utilizing the CEBAF Large Acceptance Spectrometer (CLAS), which was housed in Hall B. The CLAS detector was a large acceptance spectrometer optimized for the detection of multi-particle final states (Section 2.3). The g12 experiment collected approximately 126 TB of photoproduction data in 44 days of beam time in 2008. The center-of-mass energy for the experiment ranged from $1.77 \mathrm{GeV}<W<3.33 \mathrm{GeV}$ with a luminosity of $68 \mathrm{pb}^{-1}$.

\subsection{CEBAF}

CEBAF is composed of two linear accelerators (LINACs) connected by two semicircular arcs in which a magnetic field guides the electrons through. It utilizes a gallium arsenide photocathode laser to produce a highly polarized electron beam [24 that creates pulses of electron bunches that get injected into a LINAC. Each LINAC is composed of superconducting radiofrequency (SRF) resonant cavities (Fig. 2.2) in which a RF standing wave is established in each cavity that accelerates the electron bunch through the LINAC. The electron bunches take up to five laps around the accelerator attaining an energy of $5.714 \mathrm{GeV}$. The beam is then delivered to the experimental halls every $2.004 \mathrm{~ns}$ with an

energy spread of $\frac{\Delta E_{e}}{E_{e}} \leq 10^{-4} 25$. Upon entering the experimental halls, its polarization can be measured through a Møller polarimeter. The current delivered into Hall B for the g12 experiment ranged between 5 and $90 \mathrm{nA}$ for quality control with production runs at $65 \mathrm{nA}$. 


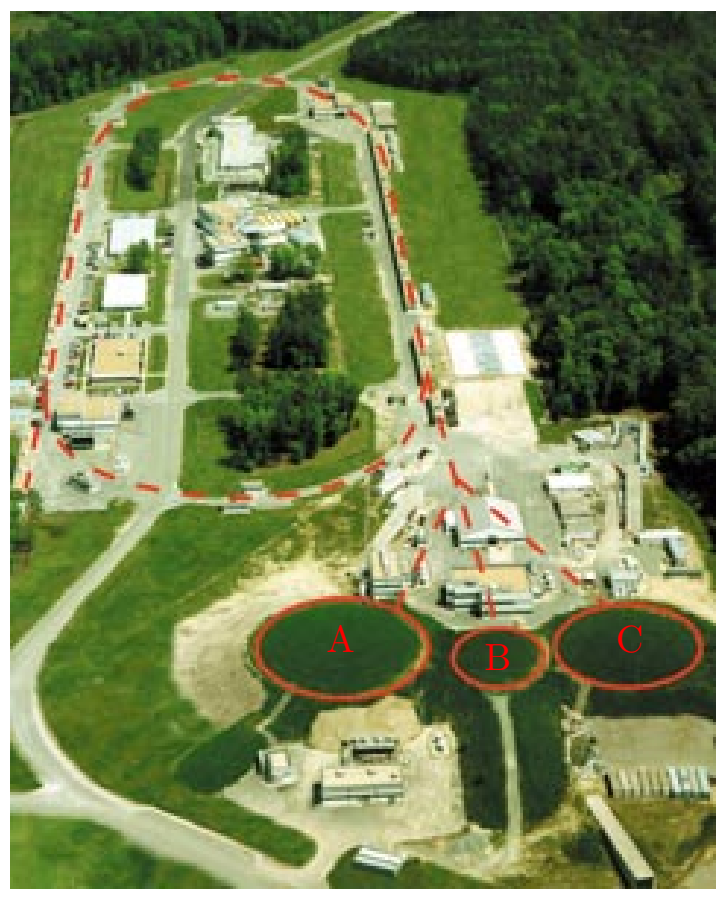

Figure 2.1: Aerial view of CEBAF prior to the construction of Hall D. Red-dashed lines show electron beam path. Source: 24

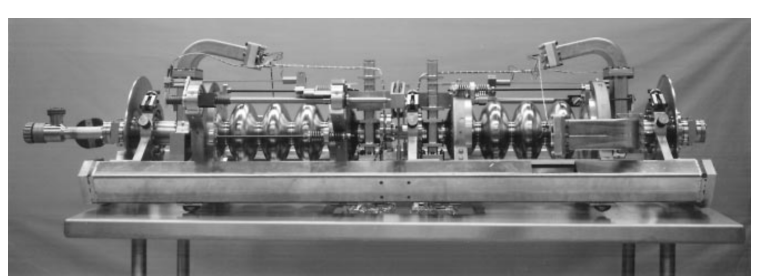

(a) CEBAF cavity pair. Source: 24

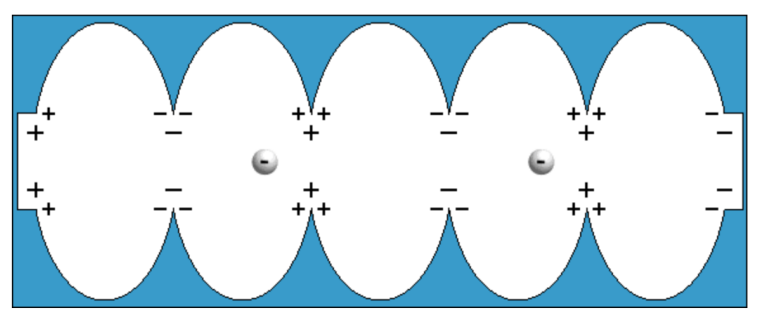

(b) CEBAF cavity diagram. Source: 26

Figure 2.2: Accelerator cavity. 


\subsection{The Tagger}

Hall B experiments can utilize either an electron beam or photon beam. The g12 experiment, specifically, utilized a photon beam. The electron beam from CEBAF is used to produce a photon beam by passing the electron beam through a radiator. As high energy electrons pass through the radiator, it interacts with nuclei and decelerates. This interaction, known as bremsstrahlung, causes the electron to lose energy, emitting a photon with energy equal to its loss. The g12 experiment used a gold foil as its radiator with a thickness of $10^{-4}$ radiation lengths ${ }^{1}$ [27]. In addition, the electrons transfer their polarization to the photons following

$$
P_{\gamma}=\frac{E_{\gamma}\left(E_{e}+\frac{E_{e}-E_{\gamma}}{3}\right)}{E_{e}^{2}+\left(E_{e}-E_{\gamma}\right)^{2}-\frac{2}{3} E_{e}\left(E_{e}-E_{\gamma}\right)} P_{e} .
$$

Equation 2.1 relates the measured polarization of the electron beam to the transferred polarization of the photon 28]. After the electrons pass through the radiator, they are bent away from the beam line by a magnet and into an array of scintillators as shown in Fig. 2.3 This magnet-scintillator system is called the tagger. As the photons are electrically neutral, they continue along the beam line towards the target. As a result, the beam is composed of only photons (Fig. $2.3 \mathrm{~b}$ ).

The tagger is composed of two layers of scintillators known as the E- and T-planes. The E-plane measures the momentum of the recoiled electrons based on the location of where the electrons were detected. The energy of the outgoing photons is then determined by conservation of energy,

$$
E_{\gamma}=E_{e \mathrm{CEBAF}}-E_{e \text { tagged }}
$$

The tagger system tagged photons of energies between $20 \%$ and $95 \%$ of the incident electron energy corresponding to photon energies between 1.142 and $5.428 \mathrm{GeV}$ with a resolution $\frac{\Delta E_{\gamma}}{E_{\gamma}} \leq 10^{-3} 25$. The T-planes provided timing measurements of the recoiling electrons with a resolution of 110 ps. These timing measurements provide a way of deducing a coincidence between the tagged photon and the electron bunch that caused it (Section 3.1).

\footnotetext{
${ }^{1}$ Mean distance over which a high-energy electron loses all but $1 / e$ of its energy due to bremsstrahlung. It is a characteristic of the material the electron is interacting with.
} 


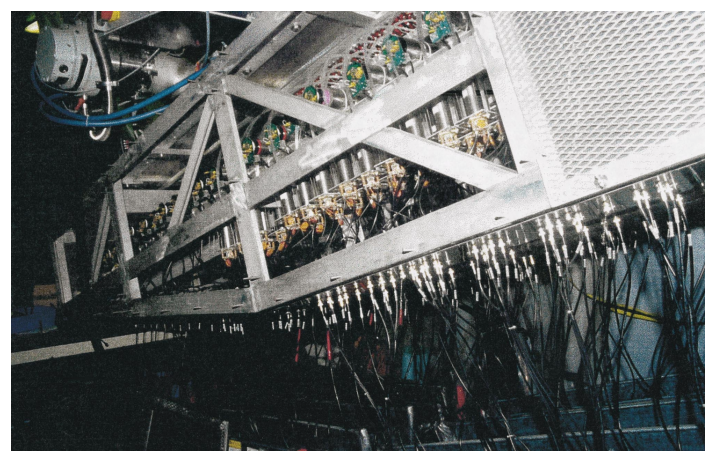

(a) The tagger with opened service panel

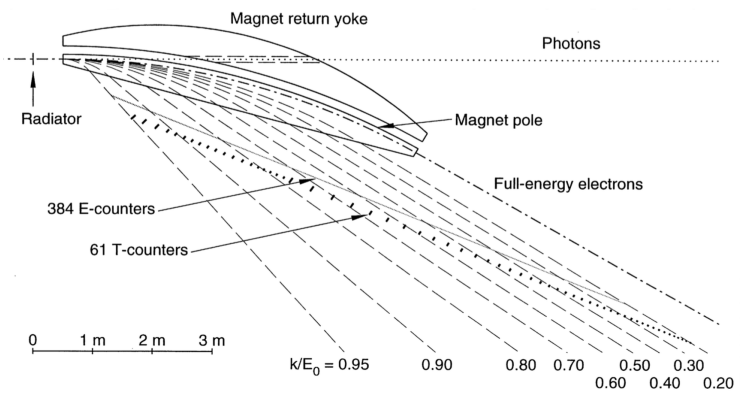

(b) Geometry of tagging system

Figure 2.3: The Hall B photon tagger. Source: 29

\subsection{The CLAS Detector}

The CLAS detector [30] is an onion-shaped detector centered around the beam line comprised of many subsystems (Fig. 2.4). A key feature of this detector, as stated in its name, is its large acceptance, which allows detection of multiple final-state particles. It is divided, azimuthally about the beam line, into six sectors by superconducting coils that produce a toroidal magnetic field. The geometry of CLAS was designed for optimal use at beam energies up to $4 \mathrm{GeV}$. The location of the center of the target that optimizes the performance of CLAS at these energies is called the nominal CLAS center. As the g12 experiment utilized higher energies, charged tracks follow straighter paths through the detector. This makes it more likely for particles to traverse through the forward hole of the detector reserved for the beam's outlet. Consequently, the target was moved upstream $90 \mathrm{~cm}$ to decrease the number of particles that would otherwise miss the detector (Section 2.4.

\subsubsection{The Start Counter}

The inner-most detector of CLAS is the start counter (ST). The ST [25] is divided into six sectors with each sector composed of four scintillator paddles. The ST obtains timing information for each track that it detects. The timing information can then be used to associate hits on the ST with the TAGR. Armed with this information, the ST can be used to select the appropriate RF time, which has the best timing resolution at approximately 


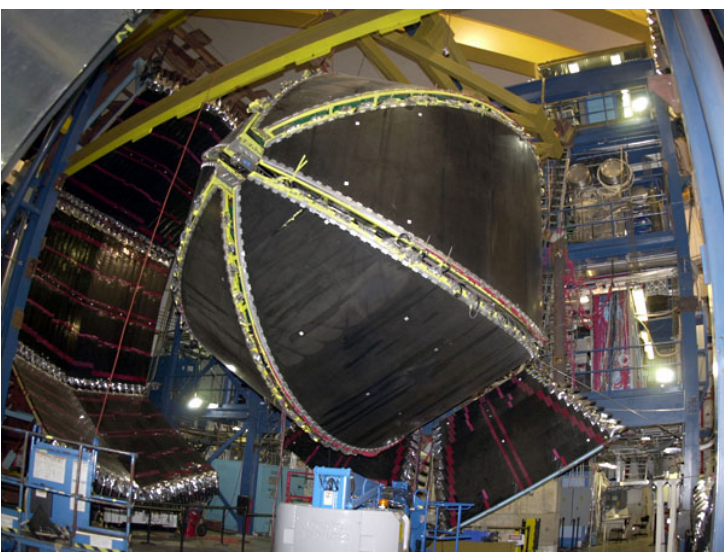

(a) The CLAS detector

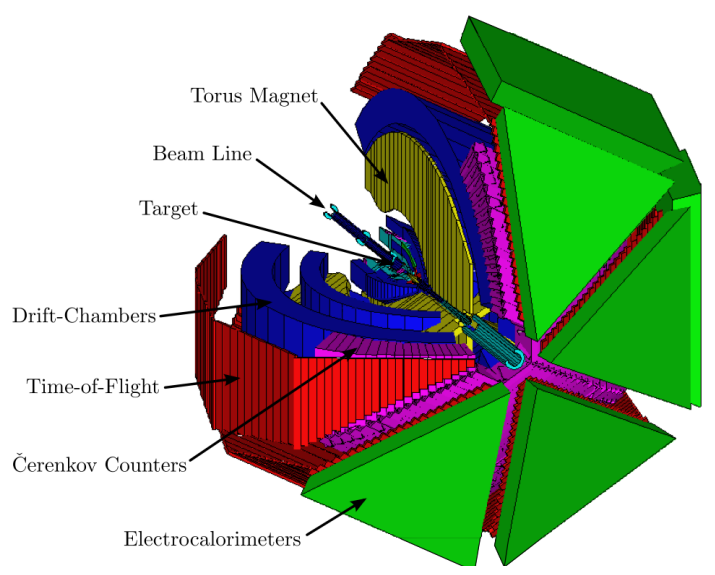

(b) Schematic of the CLAS detector

Figure 2.4: The CLAS detector. The start counter is not shown. Source: 31

15 ps. Correspondingly, the ST is used to determine an accurate measurement of a physics event's start time. In addition, the ST can be used in a variety of trigger configurations because of its segmentation (Section 2.5). The timing resolution of the start counter is approximately 350 ps.

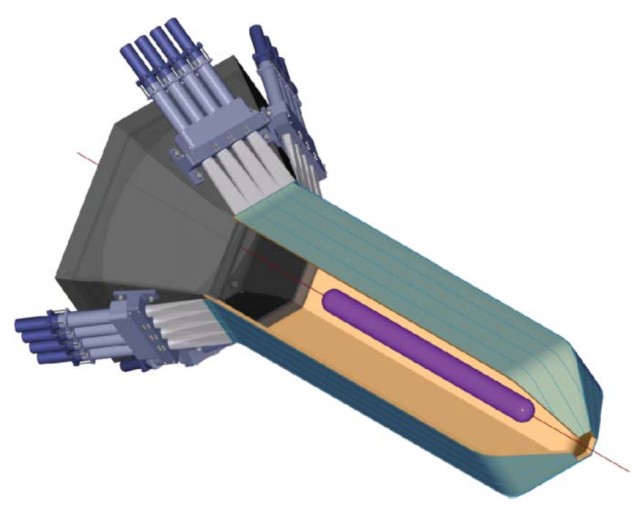

(a) The start counter

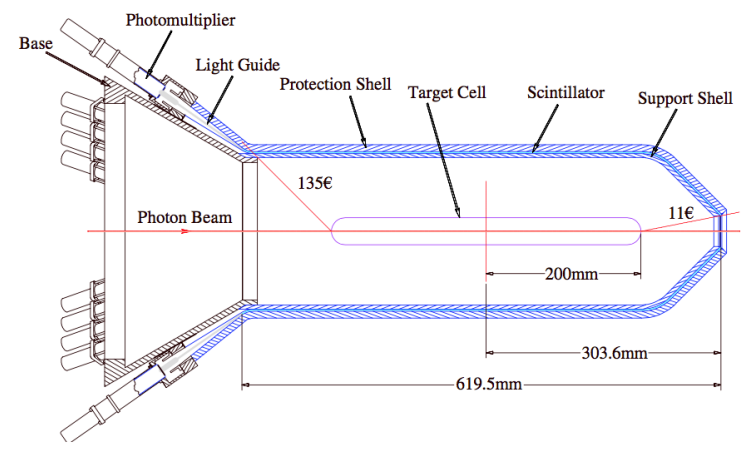

(b) Cross section of the start counter

Figure 2.5: The start counter. Source: 25

\subsubsection{The Torus}

The toroidal magnetic field is generated by six nonferrous superconducting coils (Fig. 2.6), separating CLAS into its six sectors. The coils are located between the re- 
gion 1 and region 3 drift chambers (Section 2.3.3). At the maximum design current of $3860 \mathrm{~A}$ [30], the maximum magnetic field strength the coils can provide is $25 \mathrm{kG}$. The g12 experiment utilized a magnetic field strength of $20 \mathrm{kG}$. The magnetic field direction was primarily in the azimuthal direction so that charged particles are only bent in the polar angle with respect to the beam. The field was oriented so that positively charged particles were bent away from the beam line (outbenders) while negatively charged particles were bent towards it (inbenders). This reduces the detector acceptance of negatively charged particles due to CLAS's forward hole reserved for the beam's outlet. The momentum of a charged particle can be determined by its trajectory's curvature and the magnetic field strength from

$$
p_{\perp}=q r B
$$

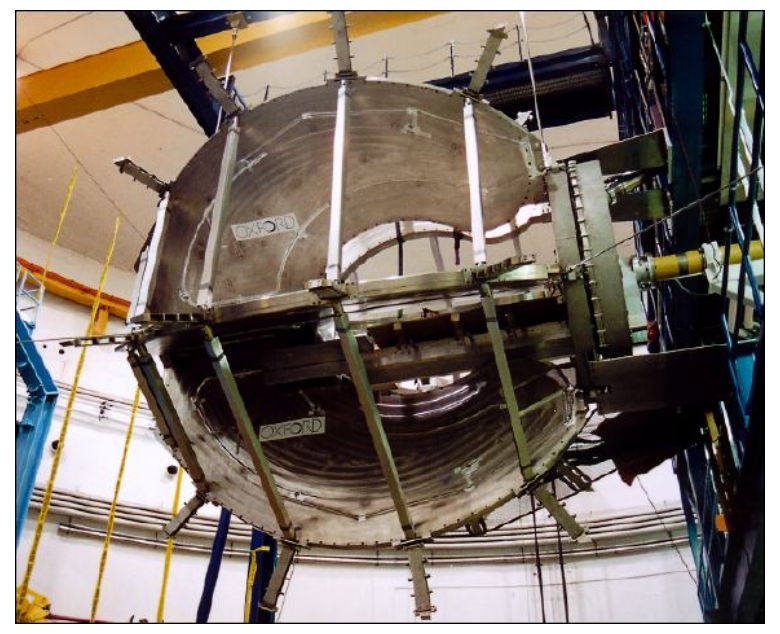

Figure 2.6: The superconducting coils. Source: 32

\subsubsection{Drift Chambers}

Following the CLAS detector's design, the drift chambers (DC) [30, 33 35] are divided into six sectors surrounding the target (Section 2.3.3). It is further separated radially into three different regions referred to as regions one, two, and three. All regions of the drift chambers contain two layers of wires known as superlayers as shown in Fig. 2.7b. One layer is axial to the magnetic field and the other is tilted by $6^{\circ}$ with respect to the first. This 
setup provides the azimuthal angle information of a track. The region two DC is situated between the superconducting coils (Section 2.3.2). As a result, it is exposed to a strong magnetic field and most of a charged particle's curvature occurs here. Region one and three DC are outside of the coils and are exposed to a weak magnetic field. A schematic showing the magnetic field strength within the DC is shown in Fig. $2.7 \mathrm{c}$.

A drift chamber cell consists of six field wires forming the vertices of a hexagon and one sense wire at the center of the hexagon (Fig. 2.7d). The cells are immersed in a $90 \%$ argon, $10 \%$ carbon-dioxide gas mixture. The field wires are run at high negative voltage while the sense wires are run at a moderate positive voltage. When a charged particle passes through the gas, the gas is ionized. Due to the potential difference of the wires, the resulting ionized electrons are accelerated toward nearest sense wires, creating a signal that is recorded. The trail of ionized electrons left behind by the charged particle allows for the reconstruction of its path and measurement of its radius of curvature in the magnetic field (Section 2.3.2). Combined with knowledge of the magnetic field, the momentum of the charged particle is then determined by Eq. 2.3.

\subsubsection{Cherenkov Counter}

The Cherenkov counter (CC) 38] is located outside of the region 3 drift chambers (Section 2.3.3) and covers angles between $8^{\circ}-45^{\circ}$ with respect to the beam line and nominal CLAS center. The detector was filled with a $\mathrm{C}_{4} \mathrm{~F}_{10}$ gas with an index of refraction of 1.00153 [30]. Cherenkov radiation occurs when a charged particle moves faster than the speed of light in a medium given by $v=\frac{c}{n}$ where $n$ is the index of refraction. Electrons and positrons, being several orders of magnitude lighter than pions, propagate faster than light in the gas with this index of refraction while pions up to $2.5 \mathrm{GeV} / c$ do not. Consequently, the Cherenkov radiation can be used to distinguish the leptons from pions. The photons emitted as Cherenkov radiation are reflected into an array of photomultiplier tubes by carefully designed mirrors as shown in Fig. 2.8. The CC was not used in this analysis. 


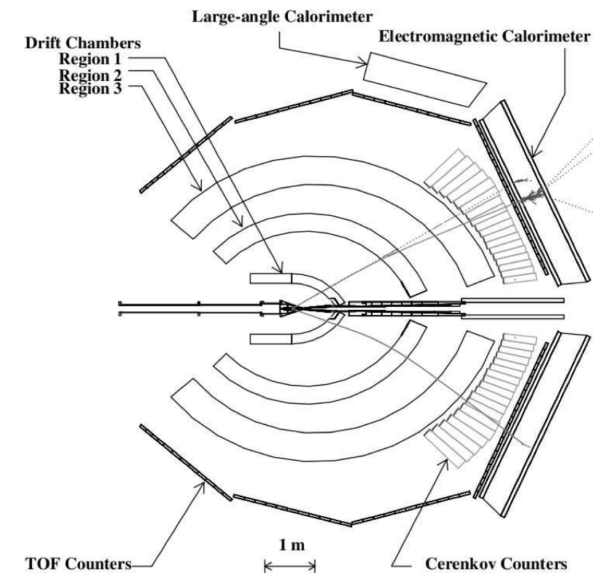

(a) Cross sectional view of CLAS along beam line. Source: 30

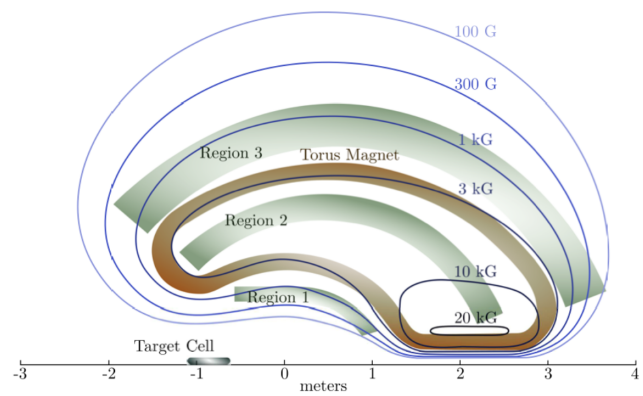

(c) Magnetic field strength within the drift chambers. Source: 37

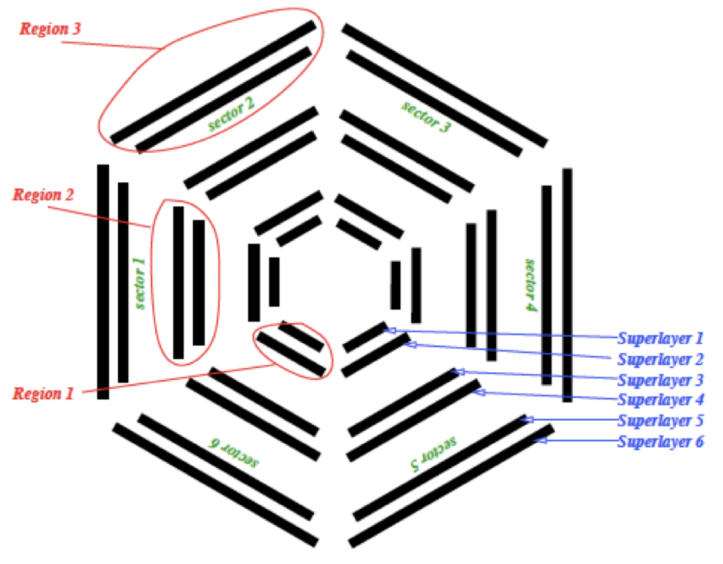

(b) Location of drift chambers relative to the the beam line. Source: 36

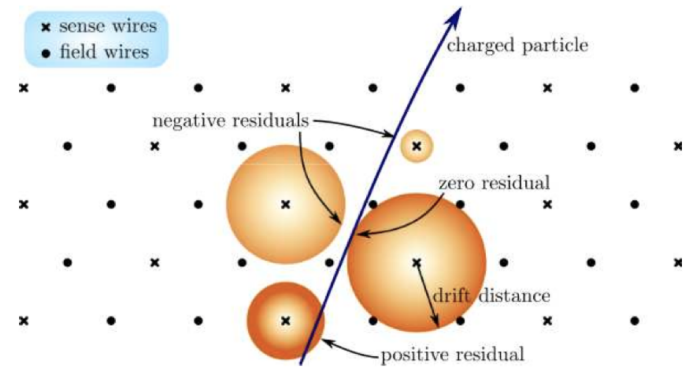

(d) Schematic of particle moving through drift chambers. Source: 37

Figure 2.7: The drift chambers system.

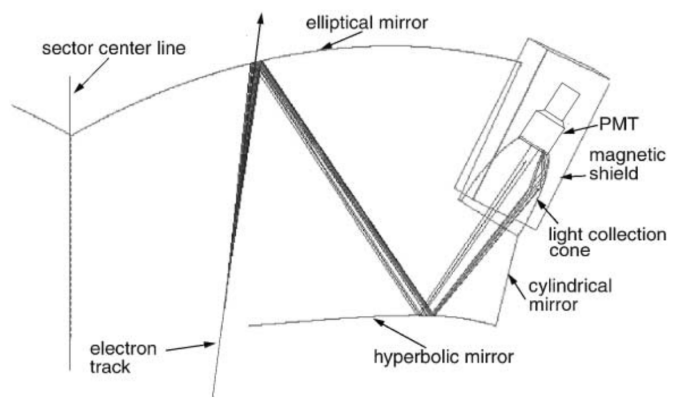

(a) Schematic of Cherenkov counter mirrors

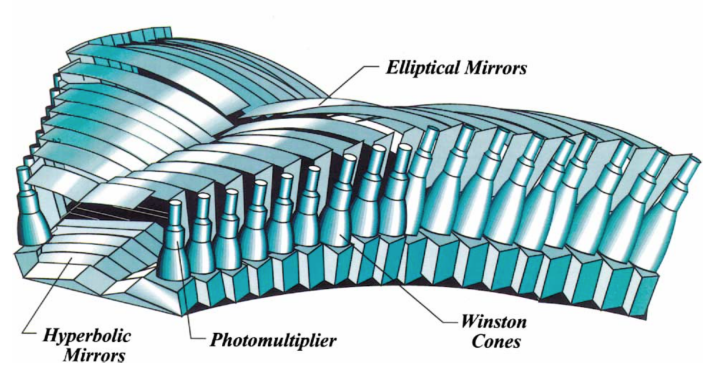

(b) Schematic of Cherenkov detector

Figure 2.8: The Cherenkov detector system. Source: 38 


\subsubsection{Time-of-Flight System}

The time-of-flight (TOF) system [39] covers angles between $8^{\circ}$ and $142^{\circ}$ with respect to the beam line and nominal CLAS center. They are positioned outside the Cherenkov counters but before the calorimeters (Section 2.3.6) as shown in Section 2.3.3. As stated in its name, the time-of-flight system is used to measure the time charged particles take to traverse the CLAS detector. It is used to determine particle velocities, which when combined with the momentum information from the drift chambers, can be used to determine particle masses following

$$
\begin{aligned}
v & =\frac{\text { Path length }}{\text { TOF }}, \\
\beta_{\mathrm{TOF}} & =\frac{v}{c}, \\
m_{\mathrm{TOF}} & =p \frac{\sqrt{1-\beta_{\mathrm{TOF}}^{2}}}{\beta_{\mathrm{TOF}}} .
\end{aligned}
$$

The timing resolution for the TOF was measured to be $150-200 \mathrm{ps}$. As the typical flight time for particles is $\sim 30 \mathrm{~ns}$, the TOF system can provide precise timing information for a given track.

Each sector of the TOF system contains 57 scintillating paddles, which are further divided into four panels. Scintillator paddles labeled $1-23$ made up panel 1 and were the most forward with respect to the beam. They were positioned $8^{\circ}-45^{\circ}$ with respect to the beam line and nominal CLAS center. Paddles $24-34$ made panel 2, paddles 35 - 45 made

panel 3 , and paddles $46-57$ made panel 4 . Several of the scintillator paddles were either inefficient, had bad timing resolutions, or were not calibrated properly. The identification of these paddles is discussed in Section 3.4 .

\subsubsection{Electromagnetic Calorimeter}

The most forward subsystem of the CLAS detector is the electromagnetic calorimeter (EC) 40, 41]. The EC has an angular coverage of $8^{\circ}-45^{\circ}$ with respect to the beam line and nominal CLAS center (Section 2.3.3). The EC consists of alternating layers of lead and 


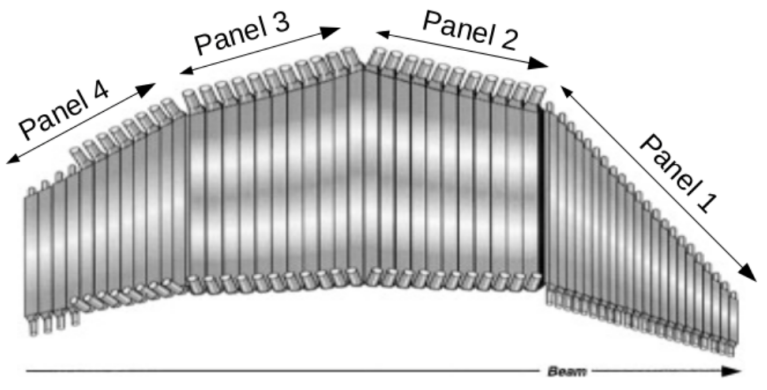

Figure 2.9: TOF paddles for one sector. Source: 39

scintillators and is divided into an inner (closest to the target) and outer stack, where the energy deposited in each stack is recorded independently. Every successive layer of scintillators is rotated $120^{\circ}$ with respect to the previous forming what are called the $U, V$, and $W$ planes. This geometric configuration makes it suitable for position measurements.

The main functions of the EC are detection and energy measurement of electrons, photons, and neutrons. The detection of electrons allows the study of key leptonic modes, such as $\omega \rightarrow e^{+} e^{-}$, while the detection of photons allows the study of reactions involving $\pi^{0}$ or $\eta$. Electrons/positrons and pions are distinguished in the EC by the characteristic of the energy deposited in the calorimeter. Electrons and positrons deposit most of their energy in the inner stack while pions deposit their energy almost uniformly throughout due to differences in their hadronic cross sections. Final state particles were identified as photons by the EC if no charged tracks were associated with an energy deposited in the EC and the velocity was greater than $0.9 c$. Likewise, particles with a velocity of less than $0.9 c$ were considered to be neutrons. The EC was not used in this analysis. 


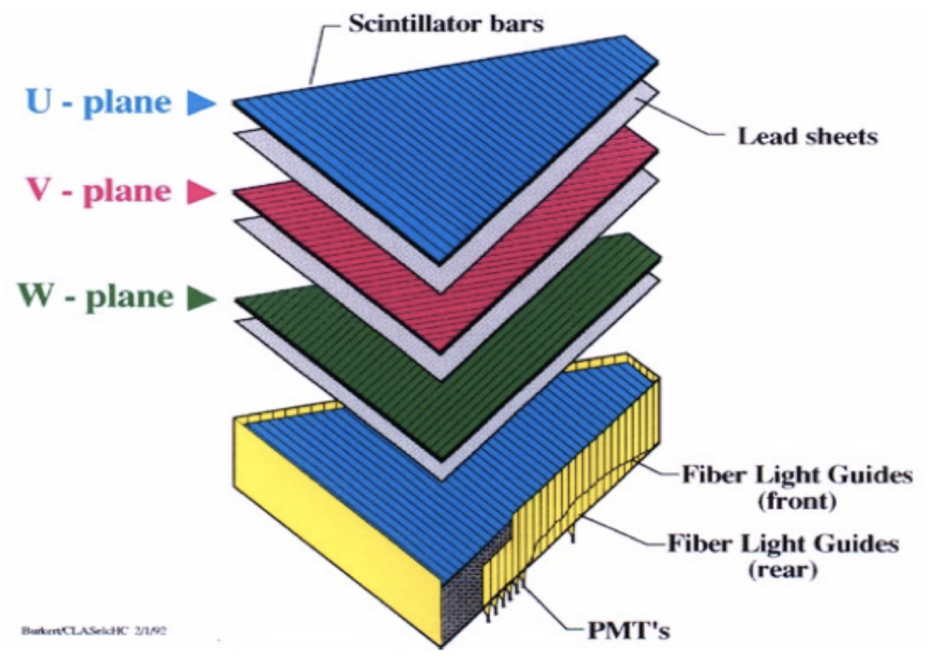

Figure 2.10: Exploded view of one of the six EC modules. Source: 40

\subsection{The Target}

The g12 experiment used an unpolarized liquid hydrogen target that was roughly cylindrically shaped with a radius of $2 \mathrm{~cm}$ and $40 \mathrm{~cm}$ in length. The target cell, shown in Fig. 2.11, was designed to hold several other materials such as deuterium and helium. As mentioned in Section 2.3, the target was located $90 \mathrm{~cm}$ upstream from nominal CLAS center. This increased the detector acceptance for small-angle tracks with respect to the beam line while decreasing it for large-angle tracks.

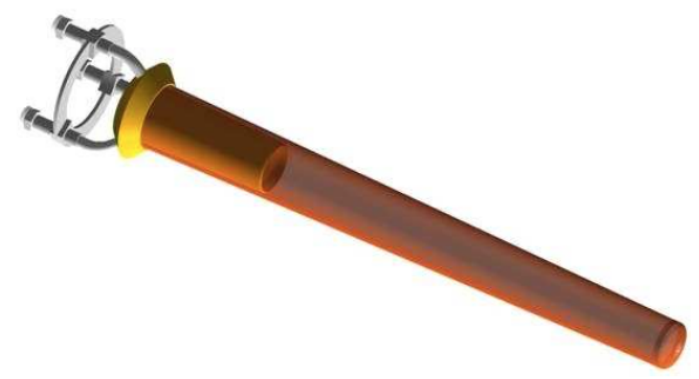

Figure 2.11: The target cell. Source: 31 


\subsection{Data Acquisition and Triggering}

Each subsystem of the CLAS detector transmits a signal to a discriminator that determines which subsystem it came from. The signals are then digitized by analog-todigital converters (ADC) and time-to-digital converters (TDC). ADC values report the voltage of the signal while TDC values report the time at which the signal arrives. In order to filter out unwanted noise, certain combinations of signals from the different subsystems in coincidence of within $100 \mathrm{~ns}$ [37] are required in order for it to be labeled a physics event. The trigger system collects the signals from all subsystems and determines which ones should be recorded based on a trigger configuration. When a signal passes the trigger configuration, the event is processed and written to magnetic tape. The g12 experiment used a field-programmable gate array (FPGA) as the trigger supervisor. This allowed for several different trigger configurations to be used and adjusted throughout the experiment.

The g12 experiment was divided into runs, which were categorized as production, diagnostic, calibration, or single-prong. The runs for g12 were labeled by numbers from 56363 - 57317. A full list of successfully reconstructed runs with the current used can be found in references 27,37 . Of these runs, the diagnostic runs mostly tested the data acquisition system and were not recorded. In addition, runs in which there were hardware failures, had less than 1 million events, or corrupt data were not recorded. The calibration runs consisted of normalization, zero-field, and empty target data. The normalization runs were used to calibrate the tagger for the measurement of the total photon flux and to check for consistency between the left and right TDC's of the tagger. The zero-field runs had the torus magnet turned off so all tracks traveled in straight lines. This was to make reconstruction (Section 2.6) of the tracks through the drift chambers easier. The empty target runs were used to determine the effects of the target walls on the production data. Production and single-prong runs were the main physics runs and consisted of $97 \%$ of the data. The differences between the production and single-prong runs were the current used (Table 2.2) and the trigger configuration. Production runs utilized a $65 \mathrm{nA}$ current while single-prong runs utilized $24 \mathrm{nA}$. 
The g12 triggering system consisted of two levels. The level-1 (L1) trigger system used signals from TAGR (Section 2.2), ST (Section 2.3.1), CC (Section 2.3.4), TOF (Section 2.3.5), and EC (Section 2.3.6). Figure 2.12 shows the L1 trigger logic for one CLAS sector. A hit in any of the four paddles in the ST and a hit in any of the 57 TOF paddles of the same sector constituted a $\mathrm{ST} \times \mathrm{TOF}$ hit. A $\mathrm{ST} \times \mathrm{TOF}$ hit is called a prong and is a track of a particle in a possible physics event. Analyses involving photons in the final state required signals from the $\mathrm{EC}$ and analyses involving leptons required both a $\mathrm{CC}$ and an $\mathrm{EC}$ hit. A hit in the tagger corresponded to a Master-Or (MOR) hit. There were two MOR triggers, MORA and MORB corresponding to different tagged photon energies. During the production runs, MORA was triggered for photon energies between 4.4 and $5.4 \mathrm{GeV}$ while MORB was triggered with photon energies between 3.6 and $4.4 \mathrm{GeV}$. During the singlesector runs, only the MORA trigger was used and was triggered at photon energies between 3.0 and $5.4 \mathrm{GeV}$. For this work, in order for a physics event to be considered, there must have been a coincidence of a MOR trigger along with two prongs in two different sectors for production runs. For single-sector runs, only a MOR trigger with a single prong was required. After the L1 trigger is satisfied, the L2 trigger was typically employed using measurements from the DC (Section 2.3.3) to make coarse tracking reconstruction and to verify the L1 trigger. A more detailed explanation on the trigger configurations and efficiencies can be found in references [27, 37]. After the trigger is completely satisfied, the event is recorded and saved to magnetic tape. 


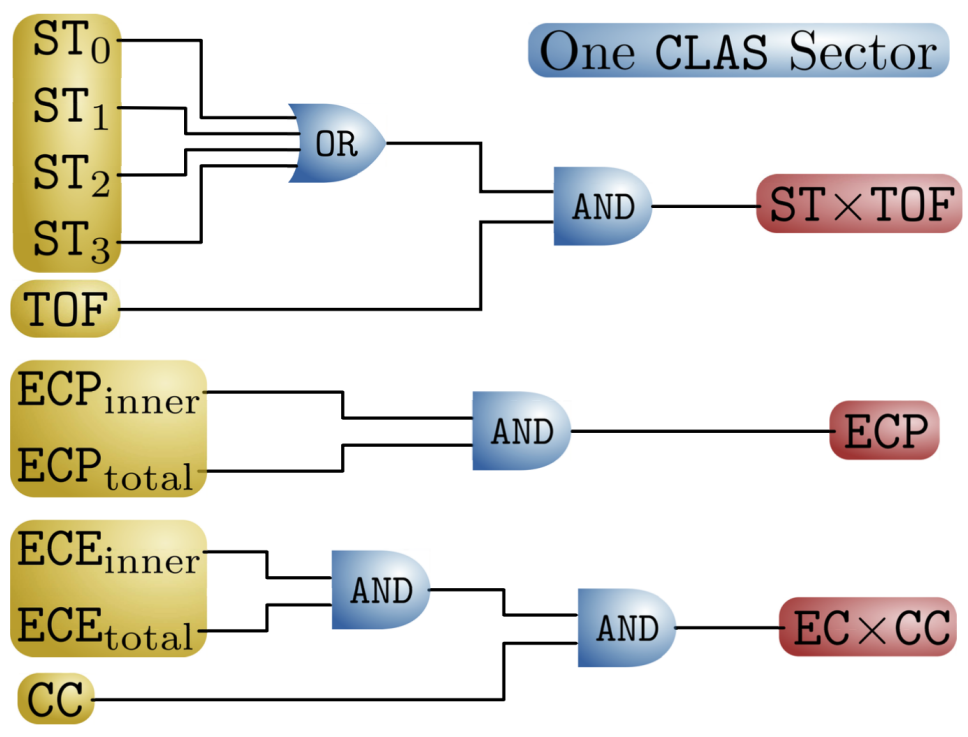

Figure 2.12: Trigger logic for one CLAS sector. The ST $\times$ TOF signal is a coincidence between any of the four start counter TDC signals with any of the 57 TOF TDC signals. ECP and ECE are the photon and electron EC thresholds, respectively. For electrons, energy must be deposited in the EC with coincidence with the $\mathrm{CC}(\mathrm{EC} \times \mathrm{CC})$. For photons, only within the EC (ECP). Source: 37

\subsection{Event Reconstruction}

The process of converting the raw data from ADC and TDC values of the detector subsystems into a suitable format for physics analyses is known as cooking and is documented in reference [37]. The toroidal magnetic field allows the event reconstruction to be done by each sector independently. The reconstruction process utilized hit-based and timebased tracking algorithms. The first step in the reconstruction is the hit-based tracking algorithm, which identifies the activated sense wires in the DC in each superlayer. It then creates track segments for each region of the DC. Tracks that aligned to physically allowable curves through each of the superlayers of the DC were selected as track candidates. The next step in the reconstruction is the time-based tracking algorithm. The time-based tracking algorithm uses the timing information of the TOF to correct for drift times inside the DC, which are then converted to drift distances. The track segments through each of the superlayers is corrected for and a new track is formed, improving the spatial resolution 
the path. Given a DC track, the radius of curvature of the track can be determined from the length of the chord and sagitta by

$$
r=\frac{s}{2}+\frac{l^{2}}{2 s}
$$

and is demonstrated in Figs. 2.7d and 2.13. From Eq. 2.3, the momentum of the particle can be determined from the radius of curvature the track makes. The mass of the particle creating the track is determined by Eqs. 2.4 to 2.6. The particle is preliminarily identified based on its measured mass as shown by Table 2.1. Refined particle identification is discussed in Chapter 3 . The run conditions for g12 are summarized in Table 2.2 .

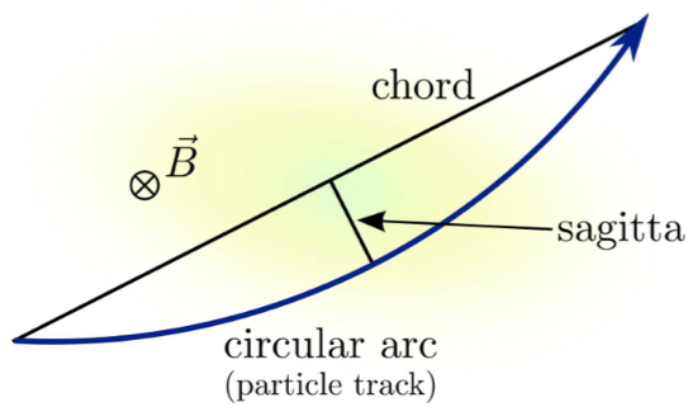

Figure 2.13: Charged particle track in DC. Particle's momentum can be determined given the chord length and sagitta. Source: 37

\begin{tabular}{l|l} 
Particle & Condition \\
\hline$\pi^{ \pm}$ & $q= \pm e$ and $m_{\mathrm{TOF}}<0.3 \mathrm{GeV}$ \\
$K^{ \pm}$ & $q= \pm e$ and $0.35<m_{\mathrm{TOF}}<0.65 \mathrm{GeV}$ \\
$p$ & $q=+e$ and $0.8<m_{\mathrm{TOF}}<1.2 \mathrm{GeV}$ \\
$d$ & $q=0$ and $1.5<m_{\mathrm{TOF}}<2.2 \mathrm{GeV}$
\end{tabular}

Table 2.1: Initial particle identification. 


\begin{tabular}{l|l}
\hline Electron Beam Energy & $5.714 \mathrm{GeV}$ \\
Electron Beam Current & $60-65 \mathrm{nA}$ (production) \& $24 \mathrm{nA}$ (single-prong) \\
Photon Beam Polarization & Circular \\
Radiator Material & $\mathrm{Au}$ \\
Radiator Thickness & $10^{-4} \mathrm{RL}$ \\
Tagged Photon Beam Energy & $1.142-5.425 \mathrm{GeV}$ \\
Target Material & $\ell \mathrm{H}_{2}$ \\
Target Length & $40 \mathrm{~cm}$ \\
Target Diameter & $4 \mathrm{~cm}$ \\
Target Position & $-90 \mathrm{~cm}$ from CLAS center \\
Target Polarization & None
\end{tabular}

Table 2.2: Running conditions for g12. 


\section{CHAPTER 3}

\section{Event Selection and Corrections}

A dominant decay mode of many excited hyperons is $Y^{*} \rightarrow p K^{-}$. The reaction chain studied in this work that contains the production of an excited hyperon is $\gamma p \rightarrow Y^{*} K^{+} \rightarrow$ $p K^{-} K^{+}$. The nonstrange reaction, $\gamma p \rightarrow p \pi^{+} \pi^{-}$, was also of interest for comparison purposes and to investigate flavor dependence of the beam-helicity asymmetry (Chapter 4). The g12 experiment collected $\approx 26$ billion events consisting of $\approx 126 \mathrm{~TB}$ of data. Much of the data recorded by the g12 experiment were from background, noise, or reactions that were not of interest for this work. From the 26 billion events, the events consisting of a $p, K^{+}$, and $K^{-}$needed to be extracted for the analysis of the $\gamma p \rightarrow p K^{+} K^{-}$reaction. Similarly, events consisting of a $p, \pi^{+}$, and $\pi^{-}$also needed to be identified for $\gamma p \rightarrow p \pi^{+} \pi^{-}$. For both reactions, all three final-state particles were required to be detected, and for there to be no missing energy or momentum. The particles in the final state of the reactions were initially identified according to Table 2.1 and events that falsely satisfied Table 2.1 were minimized following the procedures described in this chapter. Finally, the data were corrected to mitigate effects from the imperfect detectors and other effects not measured by the detectors.

\subsection{Vertex Position and Timing}

For a given event, the reconstructed vertex position is the best estimate for the location where the reaction was initialized. It is defined by the distance of closest approach to the beam line. For the aforementioned reactions, the reconstructed vertex position of the reaction was required to lie completely inside the cylinder containing the target. The target cylinder has a radius of $2 \mathrm{~cm}$ and length of $40 \mathrm{~cm}$ (Section 2.4).

The location of the reconstructed target center was first investigated. This was done by analyzing the measured $x, y$, and $z$ components of the events' vertices. The event vertex position was estimated by the point of closest approach between the three final-state tracks and the beam. It was assumed that any interaction among the final-state particles was brief 
enough to have a common vertex, i.e. no detached vertices. The $x$ and $y$ vertex components were fitted to a Gaussian around their means to estimate the central position. The central $(x, y)$ position of the reconstructed vertex position was measured to be $(-1.8,-0.9) \mathrm{mm}$ from the $z$ axis. The adjusted vertex position of the events was then given by

$$
\begin{aligned}
& x_{\text {adjusted }}=x_{\text {measured }}-(-0.18) \mathrm{cm} \\
& y_{\text {adjusted }}=y_{\text {measured }}-(-0.09) \mathrm{cm} .
\end{aligned}
$$

Events whose reconstructed vertex position was a distance greater than $2 \mathrm{~cm}$ from the line $L=\left\{(x, y, z) \subset \mathbb{R}^{3} \mid x=-0.18 \mathrm{~cm}, y=-0.09 \mathrm{~cm}\right\}$, were removed from the analysis. The $z$ component of the vertex was as expected in the range $-110--70 \mathrm{~cm}$. Figure 3.1 shows the vertex distribution and the selected region.

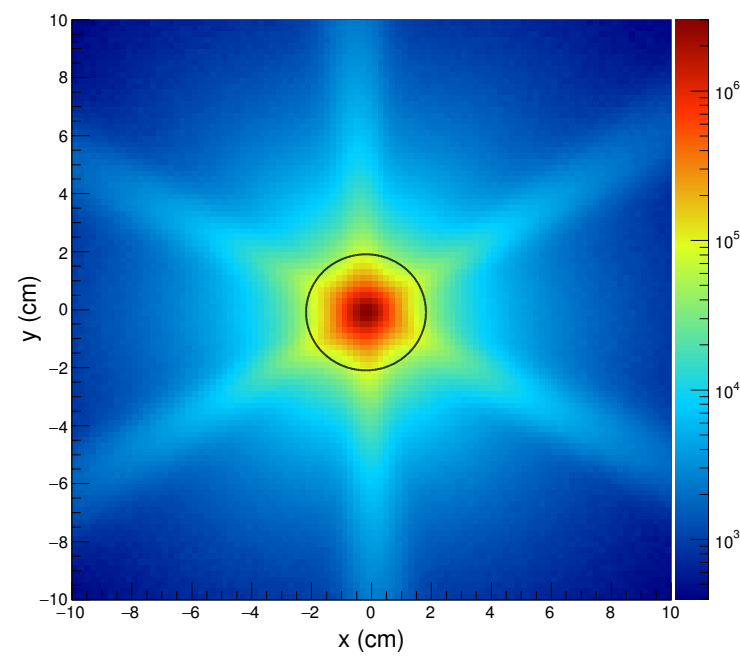

(a) $y_{\text {adjusted }}$ versus $x_{\text {adjusted }}$ component of the vertex position

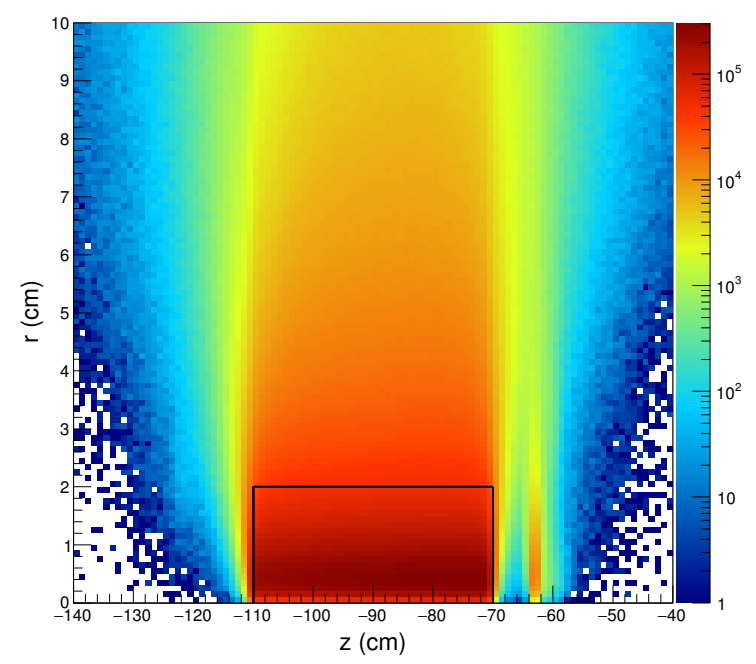

(b) $r=\left(x_{\text {adjusted }}^{2}+y_{\text {adjusted }}^{2}\right)^{1 / 2}$ versus $z$ component of the vertex position

Figure 3.1: Reconstructed vertex distributions

Second, the vertex times were investigated. As a photon is incident on the target every 2.004 ns, every event must be consistent with the incident photon that produced the reaction. The vertex time is the timing measurement corresponding to when the reaction was initialized. As the start counter (Section 2.3.1) is the closest detector to the target and,

\footnotetext{
${ }^{1}$ Recall that the target was moved $90 \mathrm{~cm}$ upstream from nominal center (Section 2.4
} 
as part of the trigger configuration (Section 2.5) was required to have a coincidence with the tagger (Section 2.2), it was used to select the CEBAF radio-frequency signal (RF) time that initiated the reaction. The closest RF time to the coincidence of the start counter and tagger was selected. The RF time serves as the most precise timing measurement available with a resolution of approximately $15 \mathrm{ps}$. The RF-corrected tagger time is given by the RF time plus the it takes a photon to reach the center of the target. The vertex time as given by the RF-tagger-ST combination is

$$
t_{\mathrm{vtx}(\mathrm{RF})}=t_{\mathrm{pho}}+t_{\mathrm{prop}}
$$

where $t_{\text {pho }}$ is the RF-corrected tagger time and $t_{\text {prop }}$ the propagation time from the event vertex to the center of the target. After a reconstructed track is given a preliminary particle type following Table 2.1, its $\beta$ is adjusted to match its assumed PDG mass [7] given by

$$
\beta_{\mathrm{adj}}=\frac{p}{\sqrt{p^{2}+m_{\mathrm{PDG}}^{2}}},
$$

where $p$ is the momentum as measured by the drift chambers (Section 2.3 .3 and Eqs. 2.3 and 2.7). The vertex time can also be measured with the TOF system (Section 2.3.5) using $\beta_{\text {adj }}$. The vertex time as measured by the TOF is given by

$$
t_{\mathrm{vtx}(\mathrm{TOF})}=t_{\mathrm{TOF}}-\frac{l_{\mathrm{TOF}}}{c \beta_{\mathrm{adj}}}
$$

where $t_{\text {TOF }}$ is the time the particle took to reach the TOF system, $l_{\text {TOF }}$ is the length of its track as determined by the time-based tracking (Section 2.6), and $c \beta_{\text {adj }}$ is its adjusted velocity. The term $\frac{l_{\text {TOF }}}{c \beta_{\text {adj }}}$ is the expected TOF of the particle assuming the identification in Table 2.1 is correct. The vertex times as measured by the RF and TOF were required to be within 1 ns of each other. Figures $3.2 \mathrm{a}$ and $3.2 \mathrm{a}$ show $\beta_{\text {TOF }}$ versus $p$ before and after applying the timing cuts. The extraneous, out-of-time bands in Fig. 3.2a are removed as a result. Figures $3.2 \mathrm{c}$ and $3.2 \mathrm{~d}$ show the effects of the timing cuts on the difference between 
the measured TOF and expected TOF,

$$
\mathrm{TOF}_{\text {measured }}-\mathrm{TOF}_{\text {expected }}=\frac{l_{\mathrm{TOF}}}{c}\left(\frac{1}{\beta_{\text {measured }}}-\frac{1}{\beta_{\text {adj }}}\right) .
$$

The extraneous bands are also removed from Fig. $3.2 \mathrm{c}$

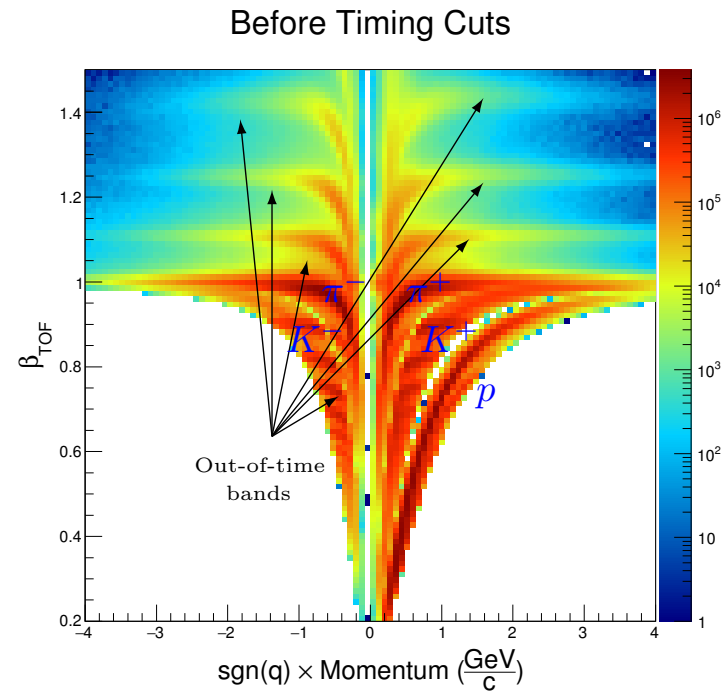

(a) $\beta_{\text {TOF }}$ versus momenta before timing cuts. Arrows are pointing to several out-of-time bands.

\section{Before Timing Cuts}

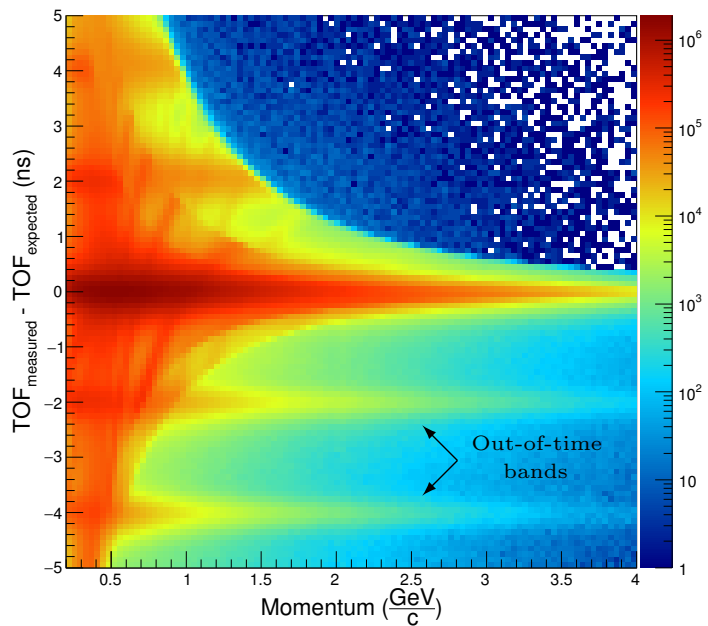

(c) $\Delta$ TOF versus momenta before timing cuts

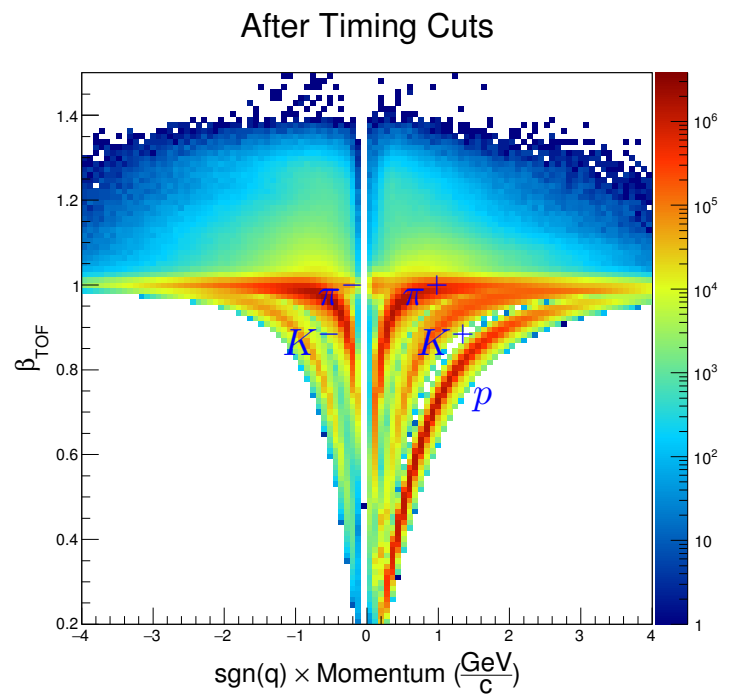

(b) $\beta_{\text {TOF }}$ versus momenta after timing cuts. Particle bands are labeled. Out-of-time bands are removed.

After Timing Cuts

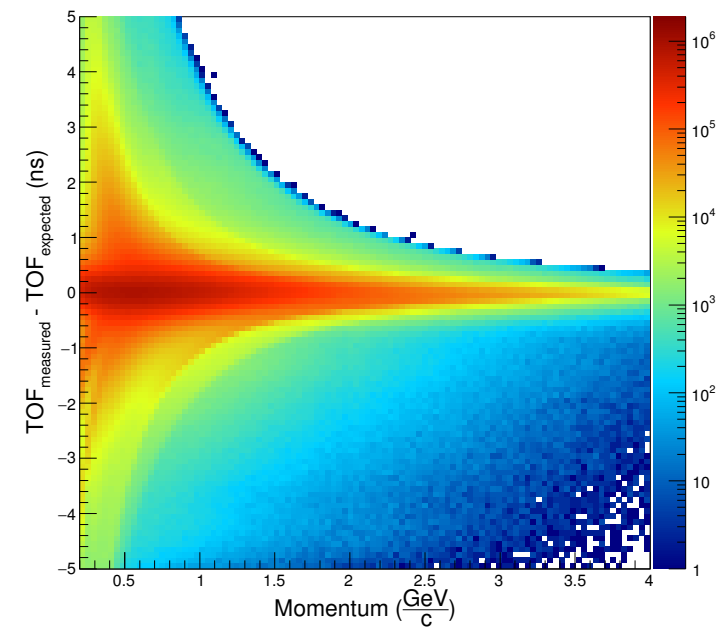

(d) $\Delta$ TOF versus momenta after timing cuts

Figure 3.2: Effects of timing cuts. 


\subsection{Multiple Photons}

As mentioned in Section 2.1, CEBAF delivers electron bunches into Hall B every $2.004 \mathrm{~ns}$, which generates tagger hits at the same interval. The production current of $65 \mathrm{nA}$ can result in multiple photons being read by the tagger for the same event. As the corresponding photons coincide within 2.004 ns from each other, they cannot be differentiated using the timing information alone. In the case where multiple photons were tagged for an event, several algorithms to select the correct photon for the event were considered: choose a photon at random, choose the more energetic photon, or eliminate events with multiple tagged photons. For this analysis, events with multiple tagged photons were removed. Figure 3.3 shows the distribution of number of tagged photons within the 2.004 ns window for all events.

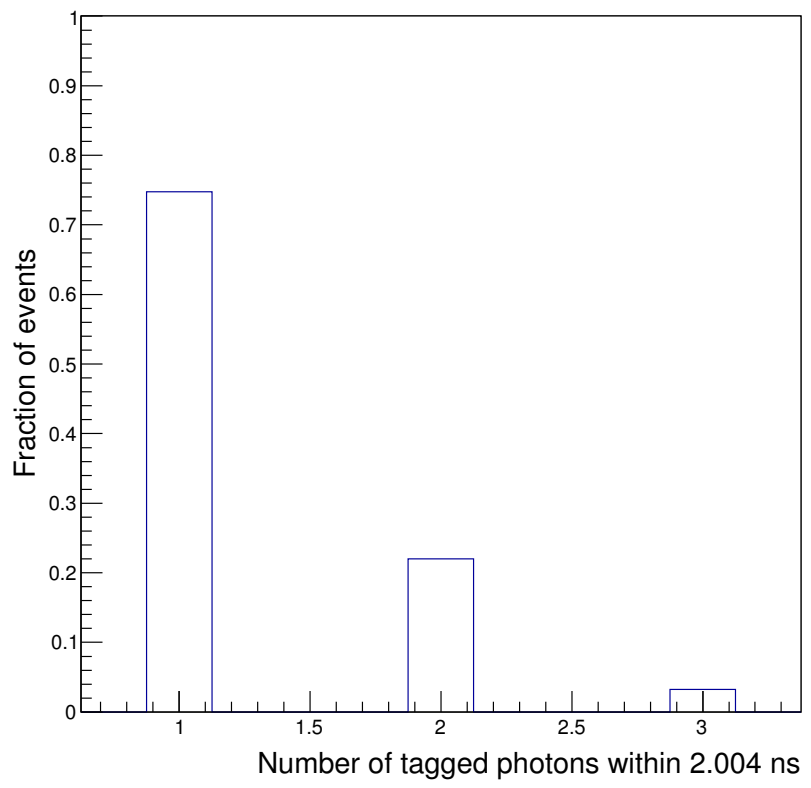

Figure 3.3: Distribution of number of tagged photons within the 2.004 ns window. 


\subsection{Fiducial Region}

Geometric fiducial cuts are used to exclude events in regions where detector acceptance changes rapidly and difficult to model. In particular, the fiducial cuts remove events whose tracks pass through the boundaries of each sector where the coils of the torus magnet are located, as shown in Fig. 3.4. The fiducial cuts for g12 were derived in reference [42]. g12's loose fiducial cuts were applied to $\gamma p \rightarrow p K^{+} K^{-}$for the partial wave analysis (Chapter 5). They were not used for the beam-helicity asymmetry analysis as acceptance effects were considered to be negligible for that analysis. Fiducial cuts allow for more accurate measurements of acceptance effects and corrections, which are applied in Section 5.4 .

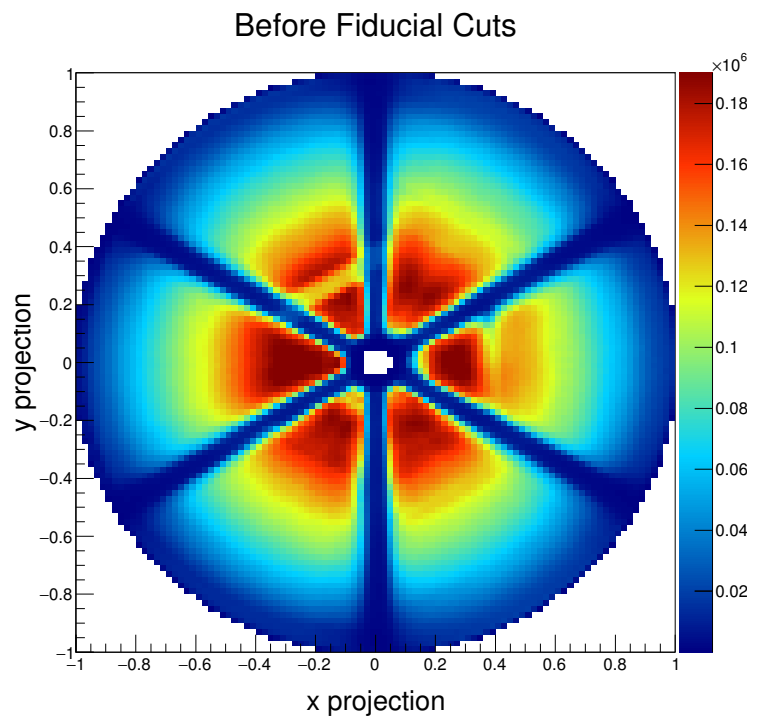

(a) Before Fiducial Cuts

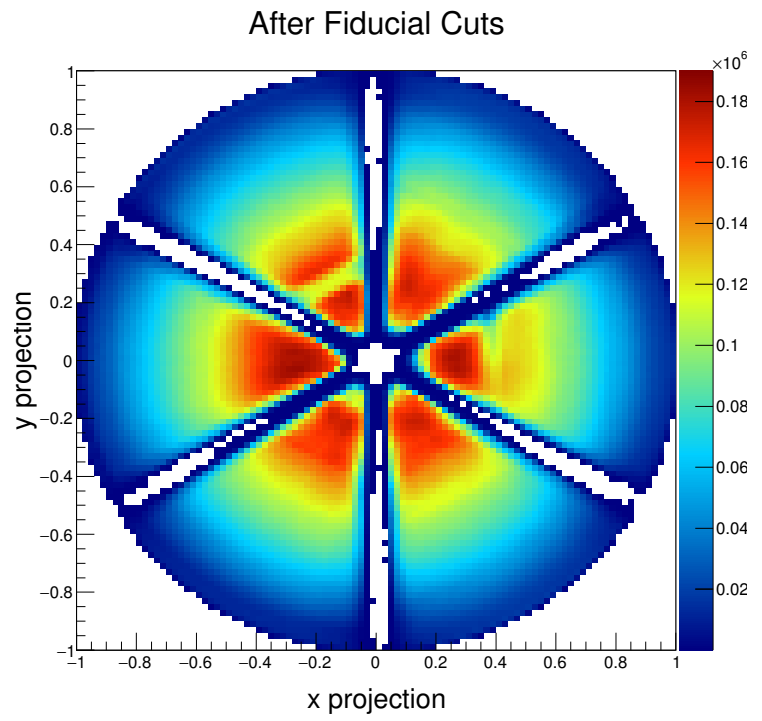

(b) After Fiducial Cuts

Figure 3.4: Effects of fiducial cuts.

\subsection{TOF Knockouts}

TOF paddles were removed from the analysis if they were considered to be inefficient. A paddle's efficiency was estimated through its relative occupancy with respect to its counterparts in the other sectors using the raw data; for paddle with ID $=x$ in sector $=y$, its occupancy was compared to the other five paddles with ID $=x$. Out of the five, the 


\begin{tabular}{lc}
\hline \hline Sector 1: & $6,25,26,35,40,41,50,56$ \\
Sector 2: & $2,8,18,25,27,34,35,41,44,50,54,56$ \\
Sector 3: & $1,11,18,32,35,40,41,56$ \\
Sector 4: & $48,19,41,48$ \\
Sector 5: & $4,5,24,33,56$ \\
Sector 6: & \\
\hline \hline
\end{tabular}

Table 3.1: Recommended list of paddles to knockout.

paddles with largest and smallest occupancies were removed. The average occupancy of the remaining three was recorded. The efficiency of paddle $x$ in sector $y$ was defined to be its occupancy divided by the average of the remaining three, as given by

$$
\text { Eff(Paddle } x \text {, Sector } y)=100 \% \times \frac{\text { Number of hits in paddle } x \text { of sector } y}{\text { Average hits of remaining three paddles }}
$$

A paddle was deemed inefficient if its efficiency was below three standard deviations from the mean efficiency. Figure 3.5 shows the relative occupancy of all paddles.

The timing resolution of each paddle was also studied as a function of run number to determine stability throughout the experiment. The data analyzed were for the $\gamma p \rightarrow$ $p \pi^{+} \pi^{-}$reaction. For the pions in this reaction, the difference between the measured TOF and expected TOF was measured for a given run and paddle and fit to a Gaussian. The resolution of that paddle for that run was estimated by the standard deviation of the Gaussian fit. This procedure was conducted for all paddles and runs. Figure 3.6 shows an example of a good and bad paddle resolution. The paddles removed due to low occupancy remove $3.06 \%$ of events per track while the paddles removed due to resolution remove $5.75 \%$ of events per track. Figure 3.7 shows the effects of the TOF knockouts. Table 3.1 shows the list of TOF paddles that were removed. Like the fiducial cuts, TOF knockouts were only applied to the $\gamma p \rightarrow p K^{+} K^{-}$reaction for the partial wave analysis. 


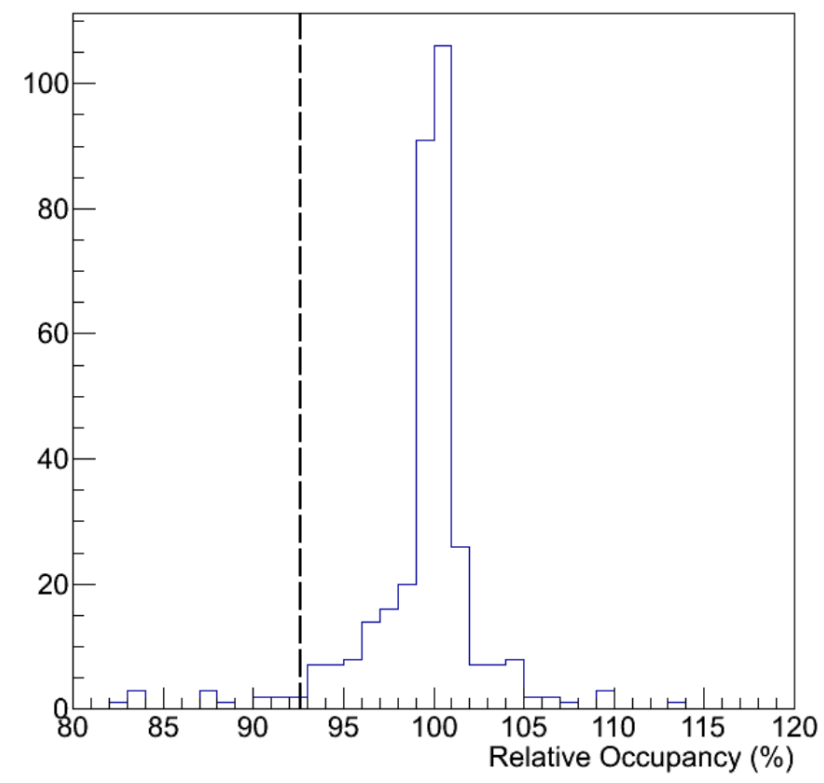

Figure 3.5: Relative occupancy of all paddles. Paddles with relative occupancy less than the dash line shown were removed from the analysis.

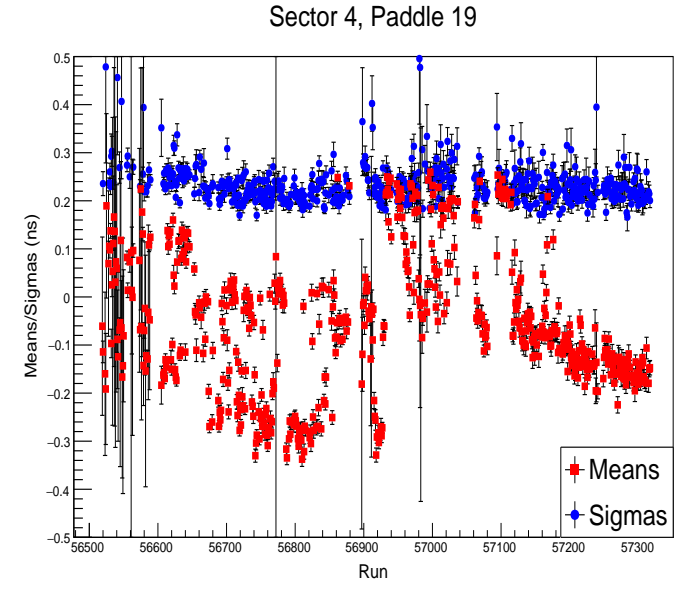

(a) Example of an unstable TOF paddle resolution.

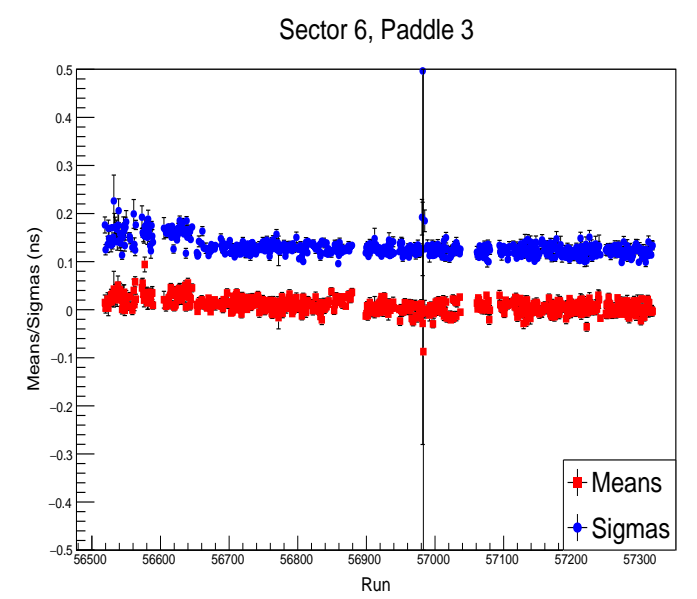

(b) Example of a stable TOF paddle resolution.

Figure 3.6: Examples of bad and good TOF paddle resolution stability. 


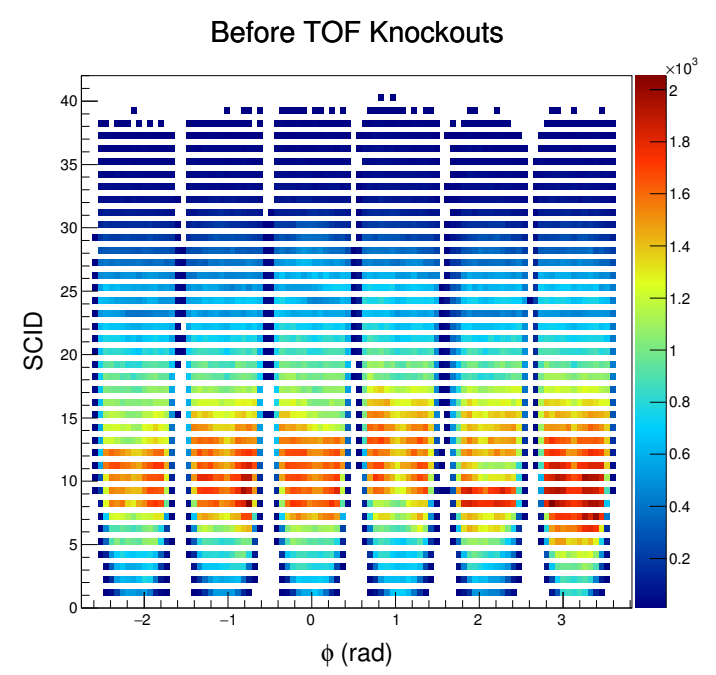

(a) Before TOFKO

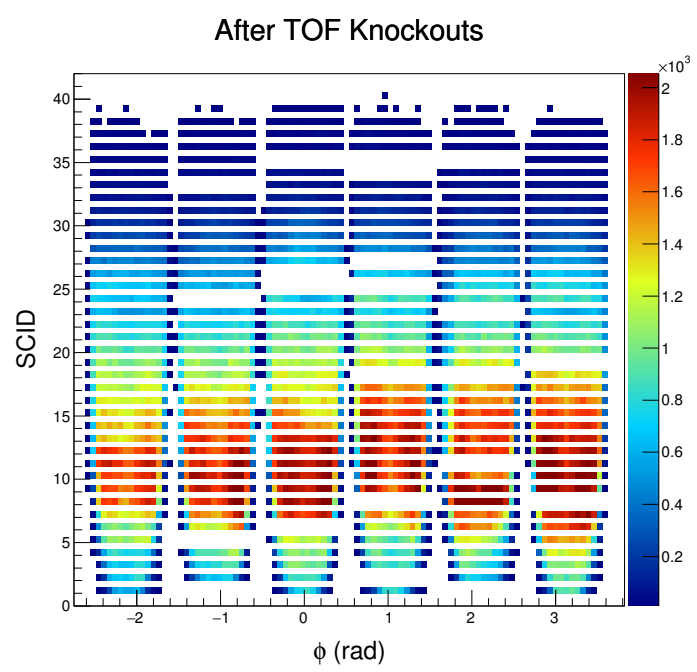

(b) After TOFKO

Figure 3.7: Effects of the TOF knockouts.

\subsection{Energy Loss}

As a particle propagates through the CLAS detector, it deposits energy through the target material and walls, beam pipe, etc. The energy that is lost is corrected for using the CLAS ELOSS software [43. The relative size of this correction is $\frac{\Delta E}{E} \sim 10^{-3}$. Figure 3.8 shows the energy loss corrections as a function of particle momentum.

\subsection{Beam Energy Correction}

It was noticed that missing masses were systematically smaller than expected for g12 and depended on the run number [27]. It was concluded in the study that the energy loss corrections were not causing nor correcting the discrepancies. Instead, it was concluded that magnetic hysteresis from the tagger magnet was responsible for the effect. Magnetic hysteresis is the phenomenon that several distinct magnetic field strengths are possible for a given current. Hysteresis occurs in ferromagnetic material, in which the relationship between the magnetic induction $\vec{B}$ and the magnetic field $\vec{H}$ is nonlinear 44 . The effect on the incident electron influences the tagged photon in turn. The correction for this effect was derived in reference 27] and its relative size is $\frac{\Delta E_{\gamma}}{E_{\gamma}} \sim 10^{-3}$. 


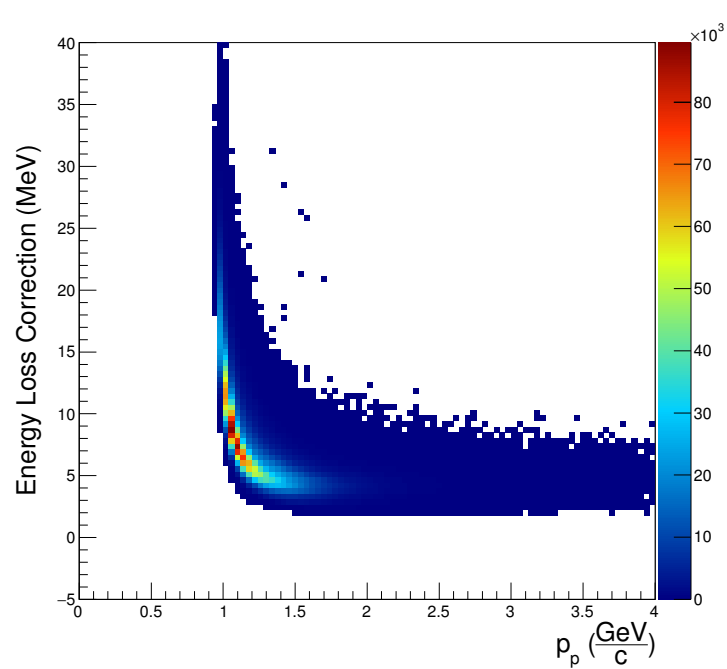

(a) Energy loss correction for proton tracks in $\gamma p \rightarrow p \pi^{+} \pi^{-}$

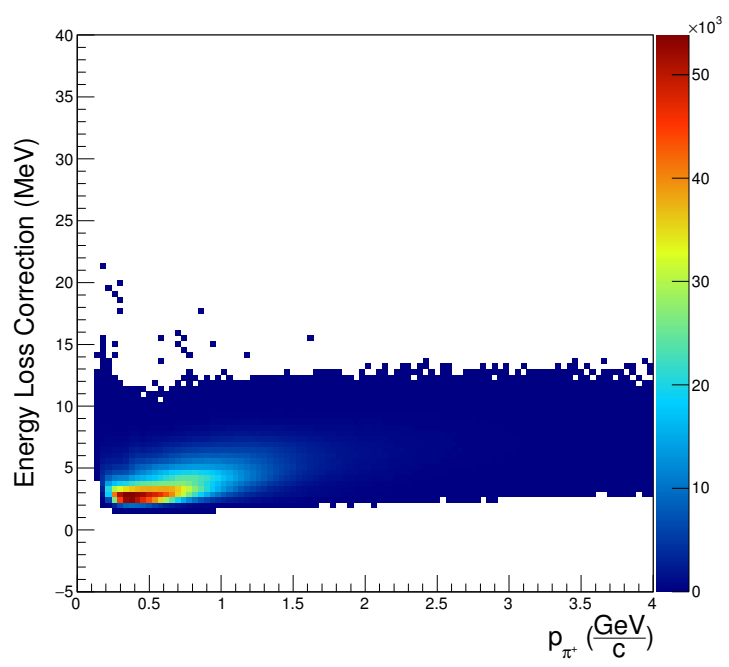

(c) Energy loss correction for positive pion tracks in $\gamma p \rightarrow p \pi^{+} \pi^{-}$

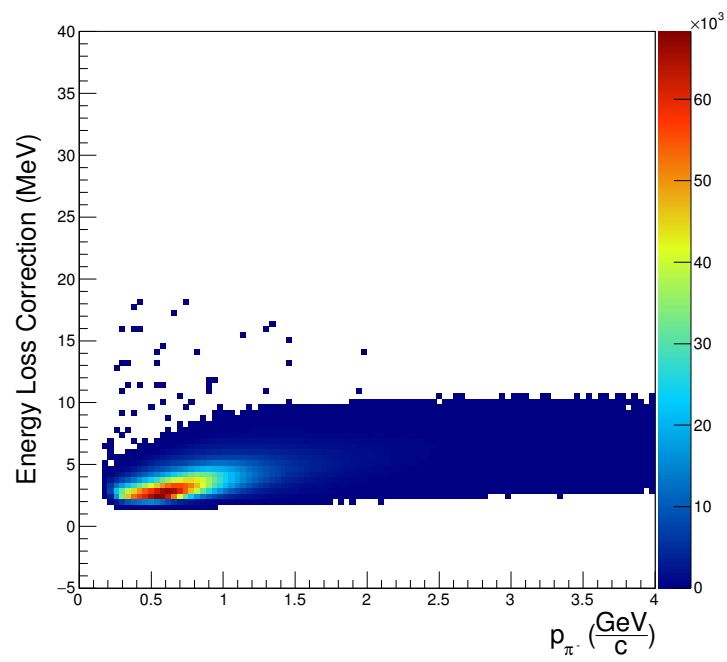

(e) Energy loss correction for negative pion tracks in $\gamma p \rightarrow p \pi^{+} \pi^{-}$

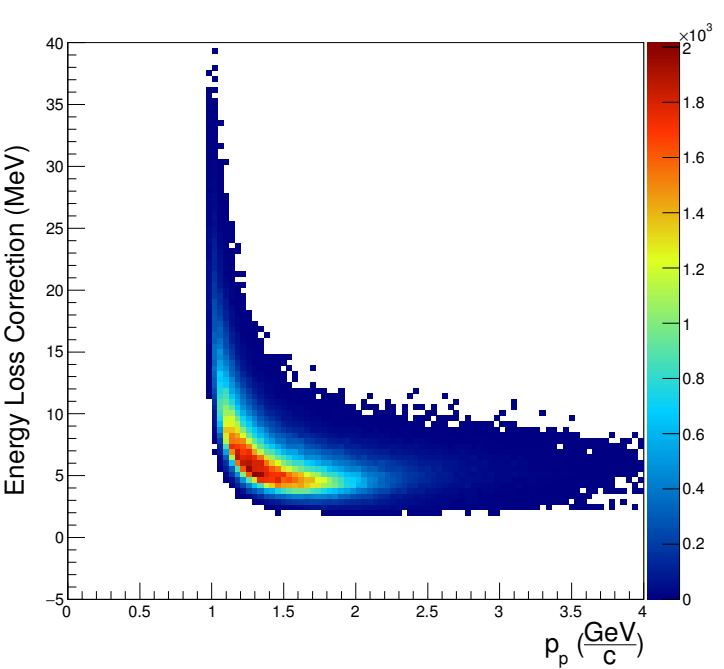

(b) Energy loss correction for proton tracks in $\gamma p \rightarrow p K^{+} K^{-}$

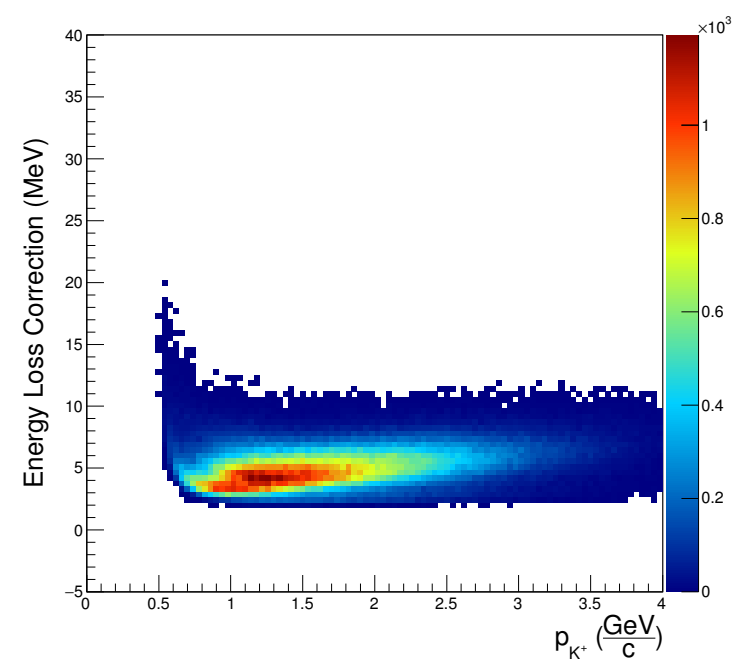

(d) Energy loss correction for positive kaon tracks in $\gamma p \rightarrow p K^{+} K^{-}$

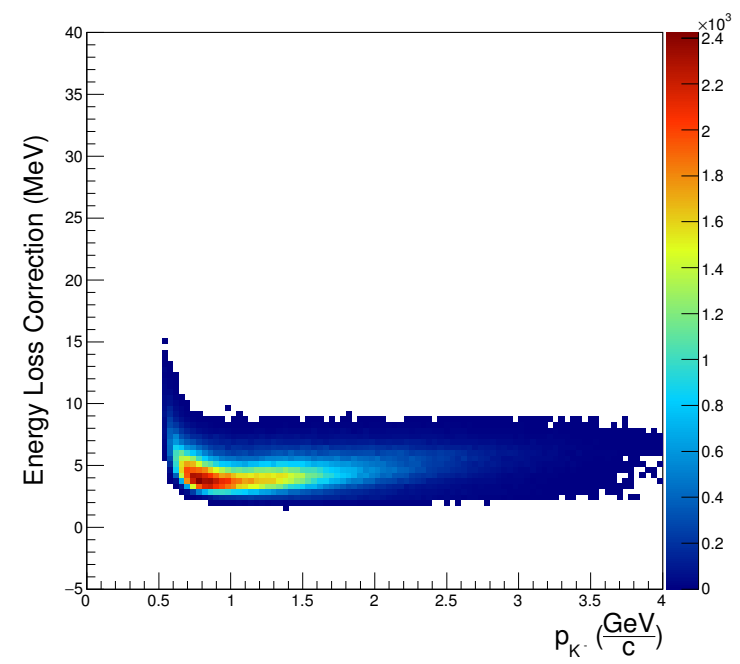

(f) Energy loss correction for negative kaon tracks in $\gamma p \rightarrow p K^{+} K^{-}$

Figure 3.8: Energy loss corrections. 


\subsection{Momentum Corrections}

The magnetic field map was calculated based upon several approximations for the areas within the CLAS detector. Consequently, the exact field map is not known and may have discrepancies with the actual magnetic field. This leads to inaccuracies in the reconstructed momenta of the particles which are corrected following the procedure in reference [45]. The relative size of this correction is $\frac{\Delta p}{p} \sim 10^{-3}$. Figures 3.9 and 3.10 shows the missing mass plots before and after all corrections are applied. The corrections yield a narrower distribution around zero missing mass and momentum.

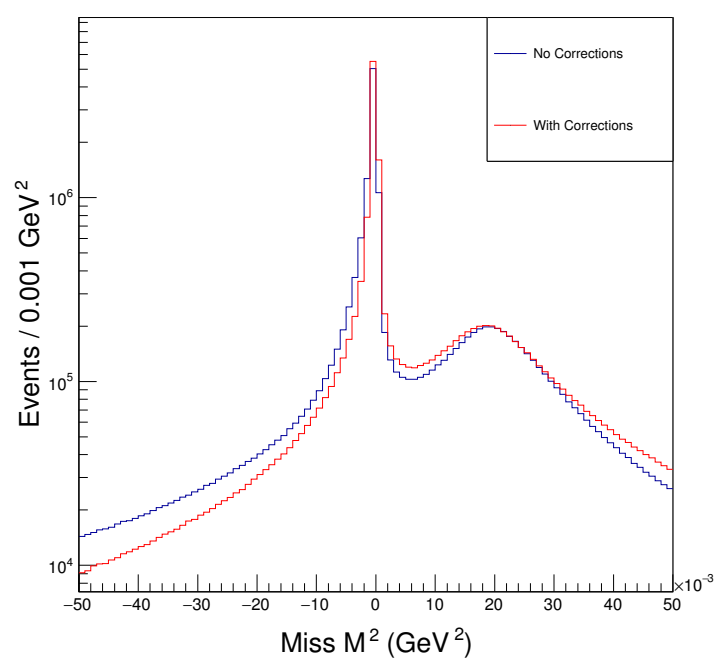

(a) Missing mass squared for $\gamma p \rightarrow p \pi^{+} \pi^{-}$

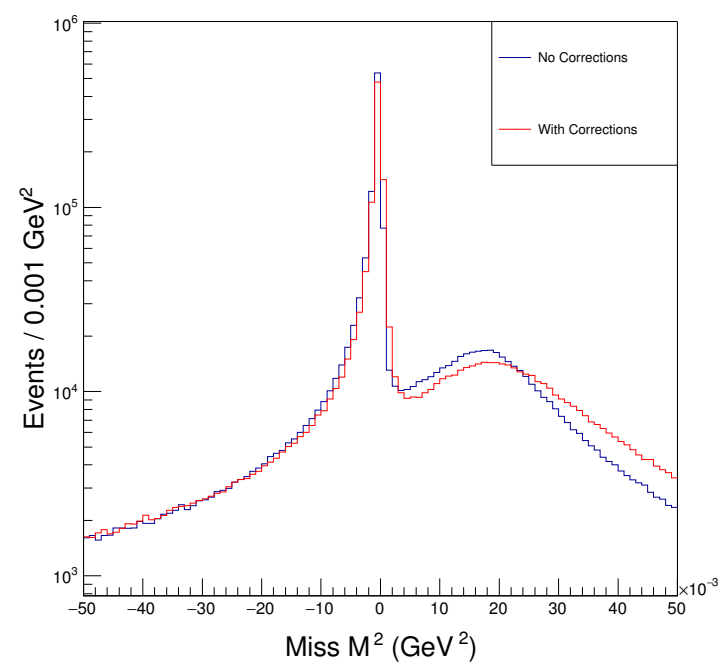

(b) Missing mass squared for $\gamma p \rightarrow p K^{+} K^{-}$

Figure 3.9: Effects of all corrections applied on the missing mass squared. 


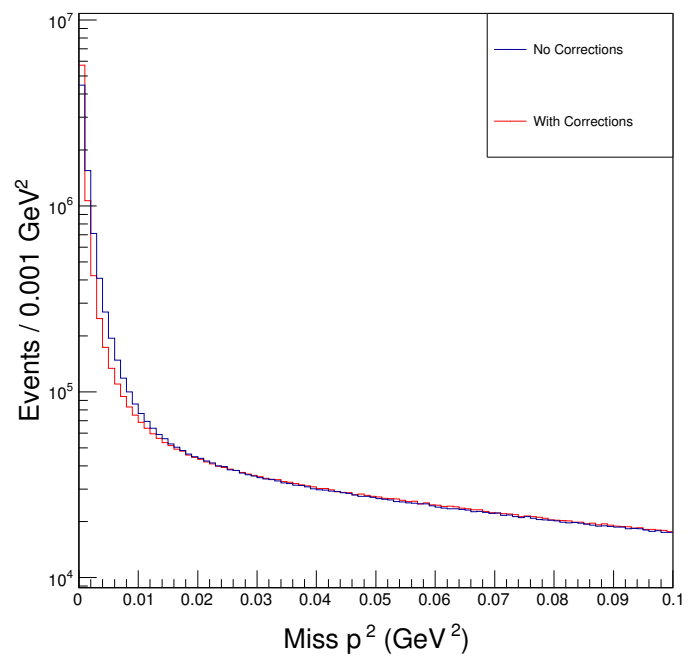

(a) Missing momentum for $\gamma p \rightarrow p \pi^{+} \pi^{-}$

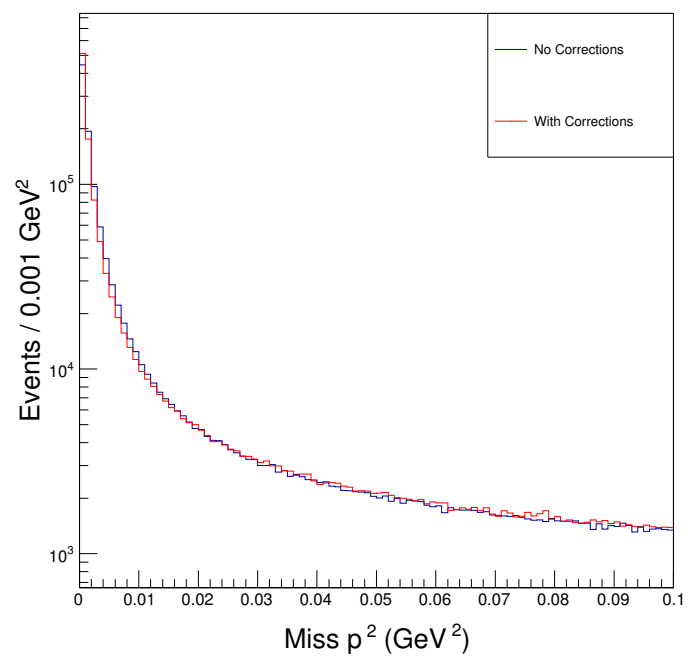

(b) Missing momentum for $\gamma p \rightarrow p K^{+} K^{-}$

Figure 3.10: Effects of all corrections applied on the missing momentum.

\subsection{Kinematic Fitting}

The final tool employed in the data cleaning process is kinematic fitting. Kinematic fitting takes as input the momentum resolution of each track in a given event and a hypothesis of what particle is responsible for each track. In addition, the constraints of energy and momentum conservation were imposed. Define $y_{0}$ as the measured energy (or equivalently, the momentum) of the photon beam and $y_{i}(i>0)$ as the measured four-vector momentum

of the $i^{\text {th }}$ particle in the final state. The fitted photon energy and four-vector momenta of the $i^{\text {th }}$ final-state particle are encoded similarly and denoted as $\eta_{0}$ and $\eta_{i}$, respectively. Let

$$
\begin{aligned}
& y=\left(y_{0}\left|y_{1}\right| \ldots \mid y_{N}\right) \\
& \eta=\left(\eta_{0}\left|\eta_{1}\right| \ldots \mid \eta_{N}\right),
\end{aligned}
$$

where $(v \mid w)$ means to augment the vector $v$ with the vector $w$, i.e., create a new, larger vector with components of $v$ followed by the components of $w$. Since $y_{0}$ has only one component and $y_{i}$ has four for each final-state particle, $y$ has $4 N+1$ components where $N$ is the number of particles in the final state. The notation $y^{i}$ will refer to the $i^{\text {th }}$ component 
of $y$ after augmentation while $y_{i}$ will refer to the four-vector momentum of the $i^{\text {th }}$ particle in the final state. This notation is used similarly for $\eta$. Given these quantities, the fitter minimizes $\chi^{2}$ given by

$$
\chi^{2}(\eta ; y)=(y-\eta)^{T} V^{-1}(y-\eta)
$$

subject to constraints

$$
\begin{aligned}
\left(0,0, \eta^{0}, \eta^{0}\right)+\left(0,0,0, m_{p}\right) & =\sum_{i=1}^{N} \eta_{i}(\text { Energy-momentum conservation }) \\
\left\|\eta_{i}\right\|_{\text {Mink. }} & \left.=m_{\text {hyp }} \text { for } i=1, \ldots, N \text { (Particle hypotheses }\right) .
\end{aligned}
$$

Here, $V$ is the covariance error matrix obtained from the track information and the superscript $T$ denotes the transpose. The fitter returns the minimized $\chi^{2}$ value and confidence level for each event, where the confidence level is given by

$$
\mathrm{CL}=\int_{\chi^{2}}^{\infty} f(x ; n) \mathrm{d} x .
$$

Here, $f(x ; n)$ is the probability density function for the $\chi^{2}$ distribution with $n$ degrees of freedom and $n=3(N-1)$. Under the conditions that the fit hypotheses are true and the uncertainties were estimated correctly, the confidence level distribution will follow a uniform distribution. For this analysis, the events were required to have a confidence level greater than 5\%, where its distribution approaches uniform as shown in Fig. 3.11. The output fourvectors are the best estimates to what the measured four-vectors should be given perfect detectors. The formalism for kinematic fitting is further elaborated in references 4648 . 


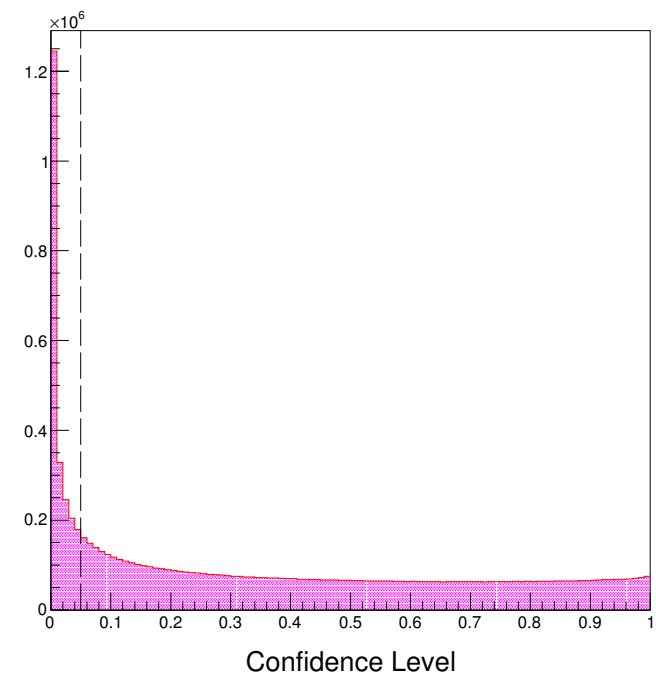

(a) $\gamma p \rightarrow p \pi^{+} \pi^{-}$confidence level distribution

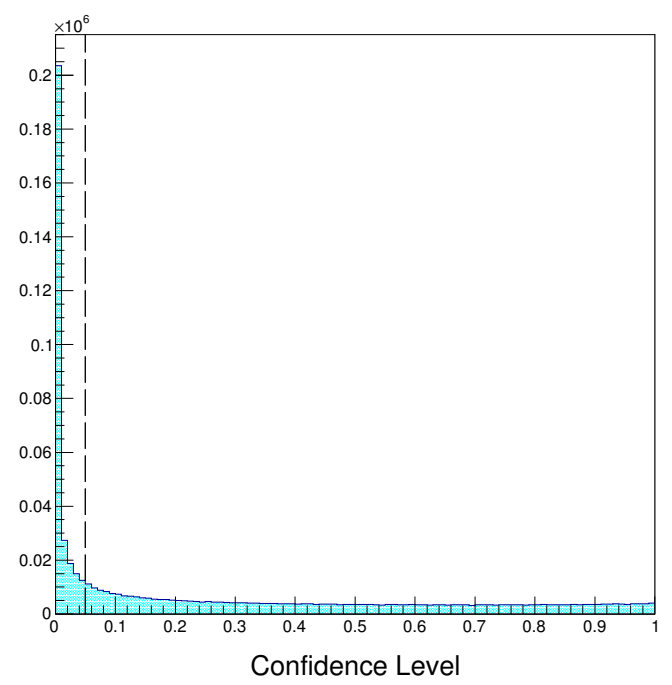

(b) $\gamma p \rightarrow p K^{+} K^{-}$confidence level distribution

Figure 3.11: Confidence level distributions.

\subsection{Summary}

The event selection and corrections applied result in extraneous events being removed. The relative sizes of the corrections applied were in the order $\sim 10^{-3}$. In addition, the background is significantly suppressed. Figure 3.12 shows the effects of all corrections and exclusivity cuts on the TOF $\beta$ versus momentum. The solid curves and dashed curves represent the theoretical value given a correct identification following Table 2.1. Figure 3.13 show consistency: the missing mass off two particles approximate the mass of the third detected particle. Figure 3.14 show the invariant mass plots after all cuts and corrections are applied. Table 3.2 summarizes the number of events remaining after each cut. 


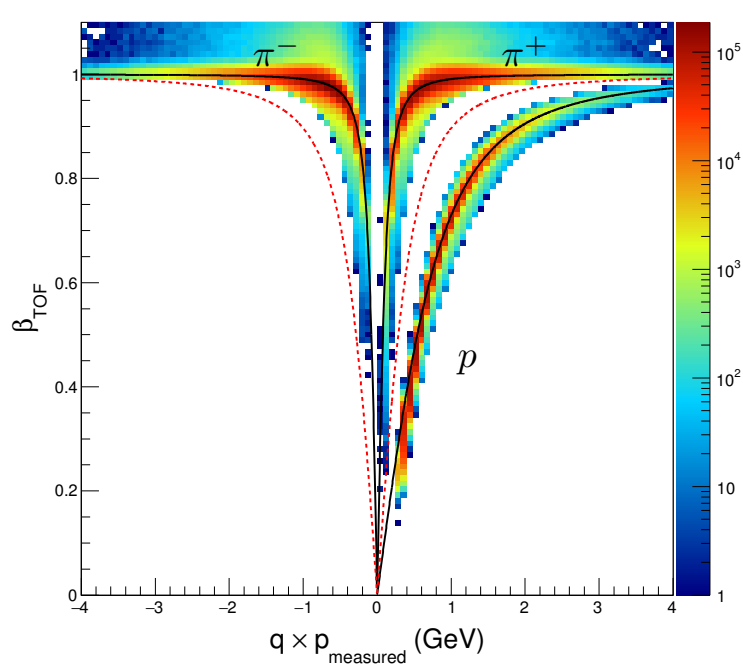

(a) TOF $\beta$ versus momentum for particles detected in pion channel. Solid, black lines represent theoretical curve for pions and protons. Dashed, red lines represent theoretical curve for kaons.

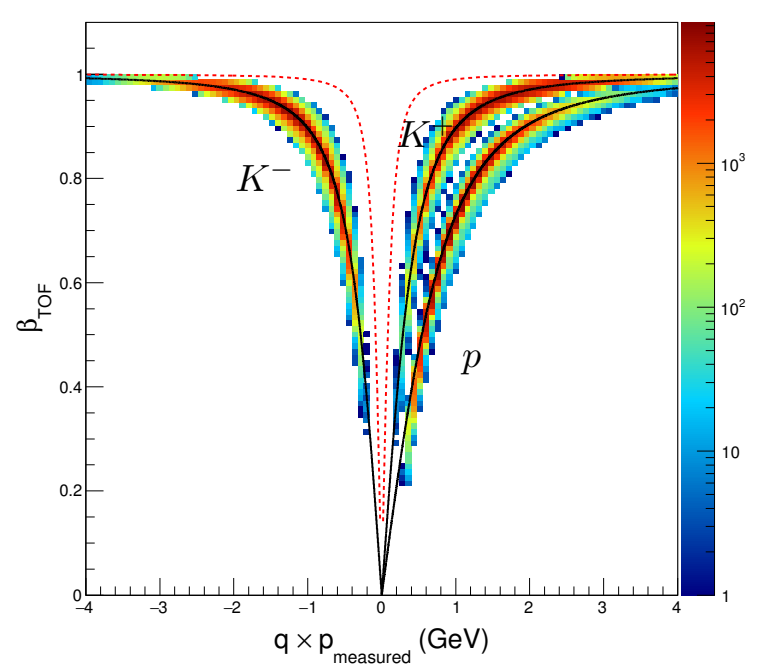

(b) TOF $\beta$ versus momentum for particles detected in kaon channel. Solid, black lines represent theoretical curve for kaons and protons. Dashed, red lines represent theoretical curve for pions.

Figure 3.12: TOF $\beta$ versus momentum after all cuts and corrections applied.

\begin{tabular}{|l|r||r|}
\hline \multicolumn{1}{|c|}{ Cut } & \multicolumn{2}{|c|}{ Events After Cut } \\
\hline & Kaon Channel & Pion Channel $^{1}$ \\
\hline Initial Skim & 28815866 & 123809904 \\
Vertex Position & 12640961 & 51431587 \\
Vertex Timing & 2807313 & 45160908 \\
Multiple Photons & 2091078 & 38795528 \\
Missing Momentum & 677773 & 9377275 \\
Confidence Level & 400728 & 7175157 \\
\hline
\end{tabular}

Table 3.2: Summary of data reduction.

$1 \approx 15 \%$ of dataset 


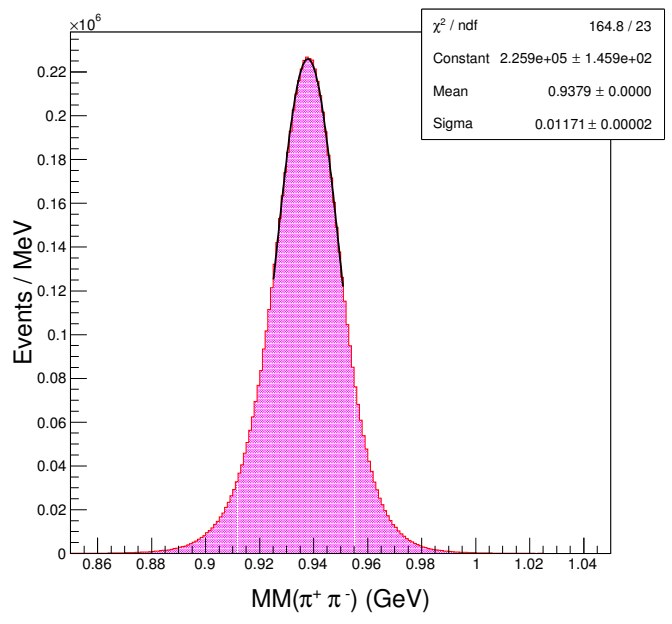

(a) Missing mass off $\pi^{+} \pi^{-}$

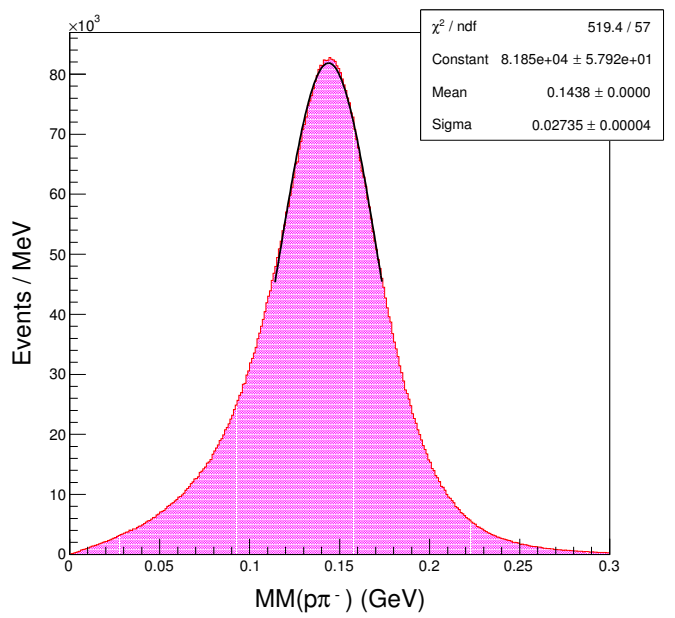

(c) Missing mass off $p \pi^{-}$

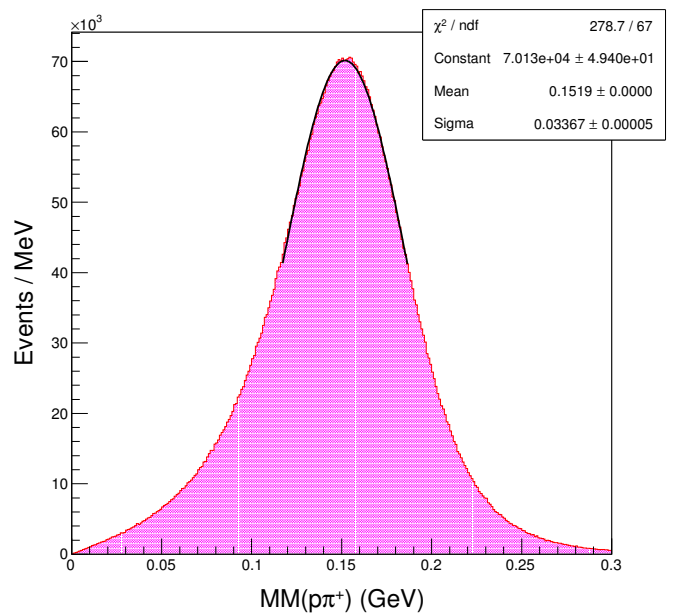

(e) Missing mass off $p \pi^{+}$

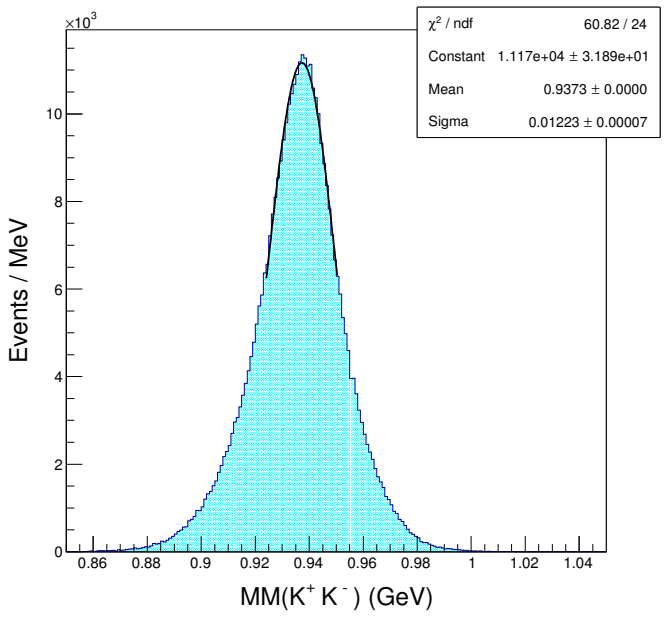

(b) Missing mass off $K^{+} K^{-}$

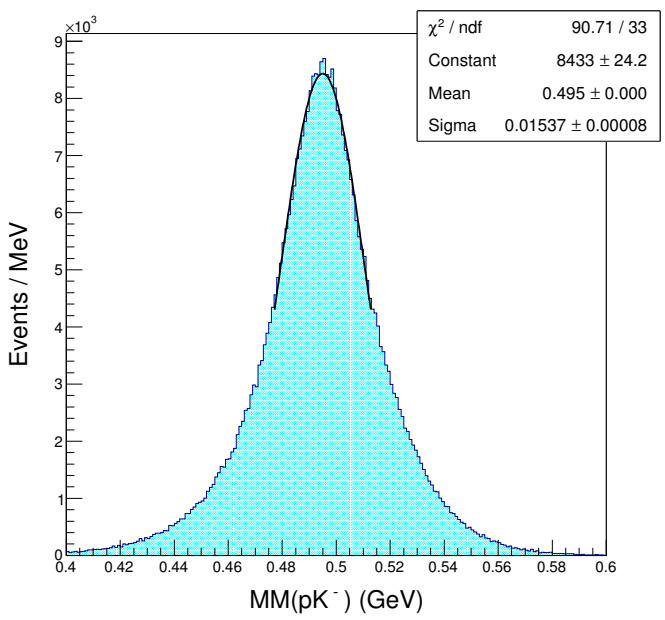

(d) Missing mass off $p K^{-}$

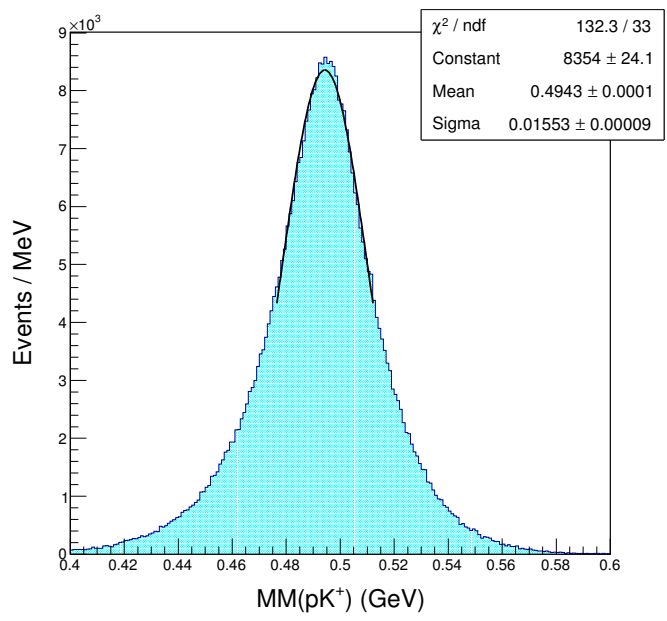

(f) Missing mass off $p K^{+}$

Figure 3.13: Missing mass consistency plots after all cuts and corrections applied. 


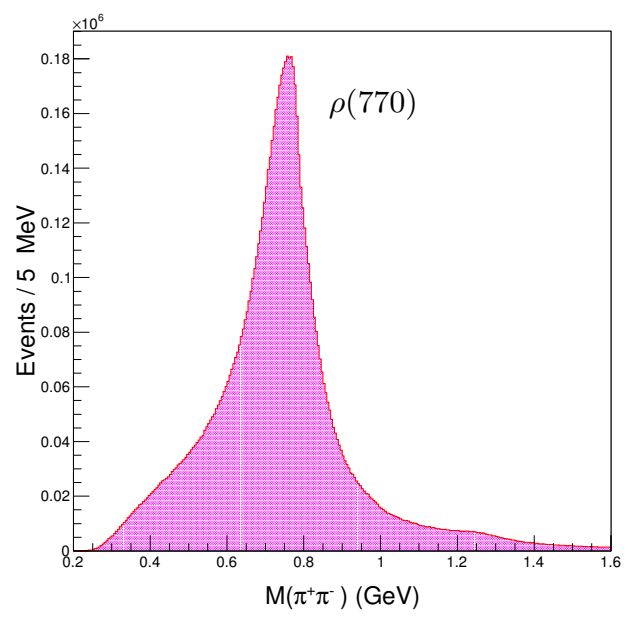

(a) Invariant mass of $\pi^{+} \pi^{-}$

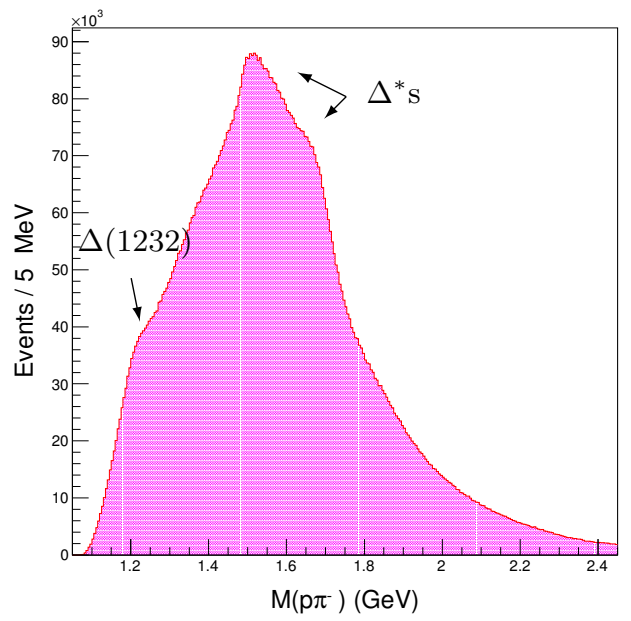

(c) Invariant mass of $p \pi^{-}$

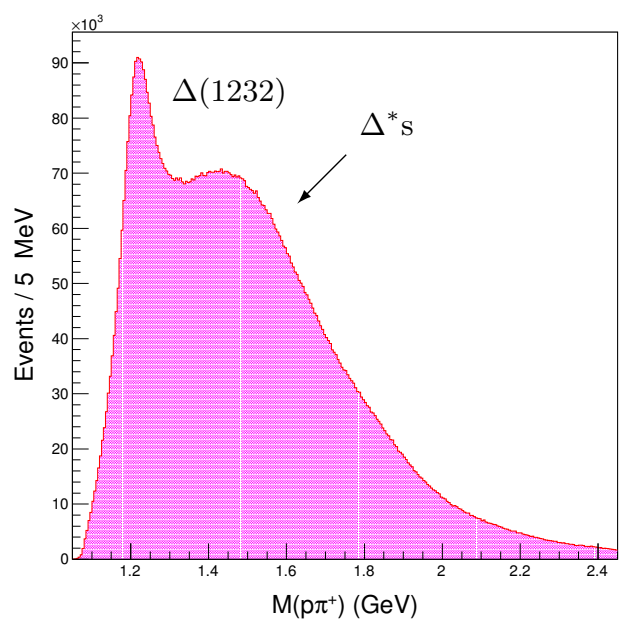

(e) Invariant mass of $p \pi^{+}$

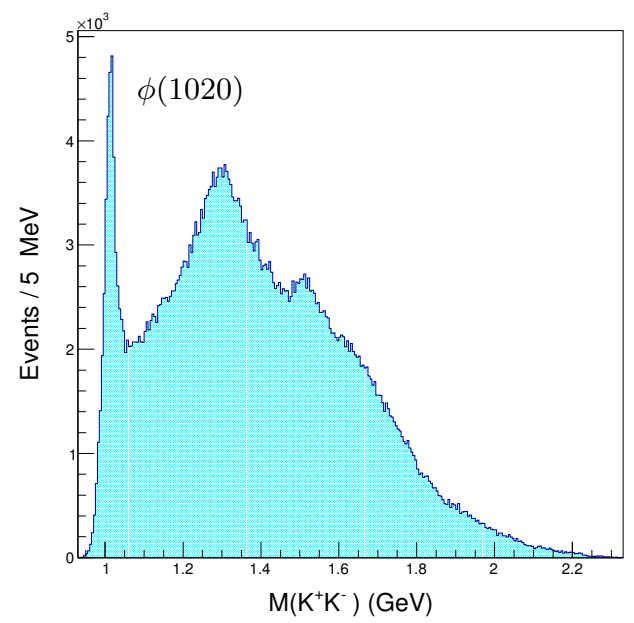

(b) Invariant mass of $K^{+} K^{-}$

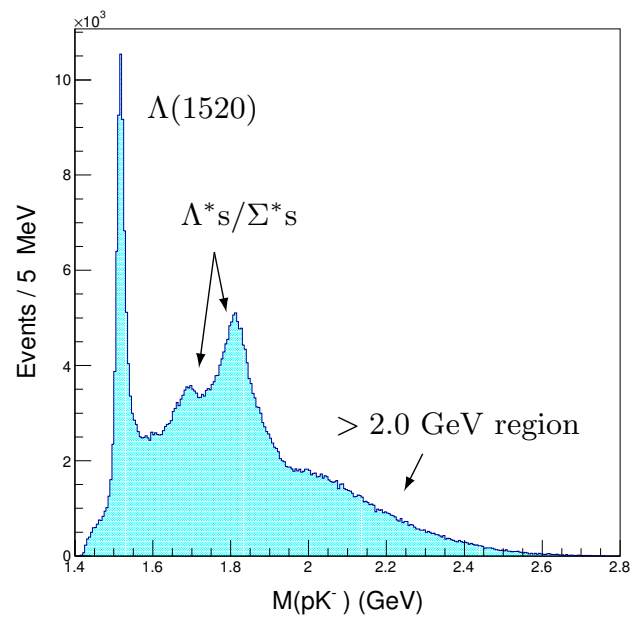

(d) Invariant mass of $p K^{-}$

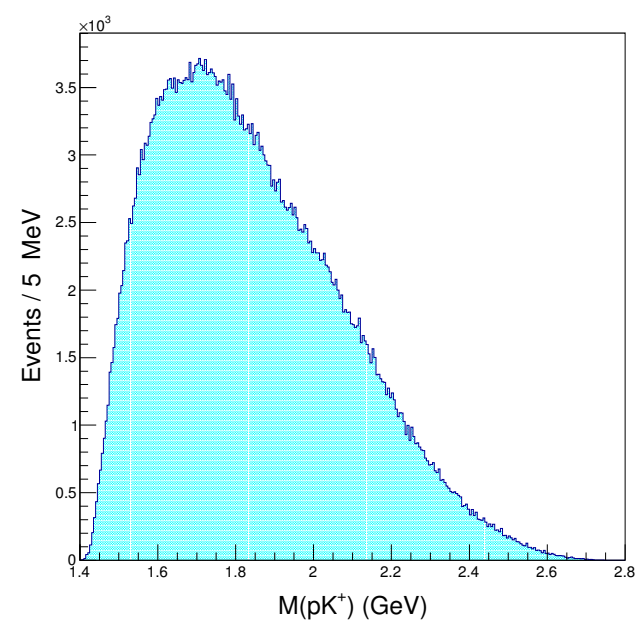

(f) Invariant mass of $p K^{+}$

Figure 3.14: Invariant mass plots after all cuts and corrections applied. 


\section{CHAPTER 4}

\section{Beam-Helicity Asymmetry}

As mentioned in Chapter 1, one of the main problems in nuclear physics is the understanding of the nucleon's structure. The constituent quark model (CQM) is a QCD-inspired model that attempts to describe the spectra of hadrons. The properties of the excited states reflect the structure and the relevant degrees of freedom within them. However, many of the excited states predicted by the CQM have not been confirmed experimentally. The difficulty in determining the relevant degrees of freedom is further exacerbated by the complex underlying production mechanisms.

The understanding of the production mechanisms of hadrons currently relies on an effective Lagrangian approach. The parameters of these models are either taken to be free or are constrained by experimental data. Polarization observables are sensitive probes of hadronic processes and are essential to constrain the parameters of these models. They are also needed in the interpretation of a given reaction in terms of the various resonances that contribute; cross section measurements along with polarization observables are used to extract amplitudes for each contributing process, which are interpreted as arising from a number of resonant and nonresonant contributions [17].

Polarization observables arise from the different spin orientations from the incident beam, target, or recoil particle in a reaction. They give rise to asymmetries in the cross section in certain kinematic regions, which can be measured. Reference 17] contains the general reaction model for three-body final states with the definitions of each polarization observable arising from the different spin orientation combinations of the reaction. Polarization observables are notoriously difficult to model, as demonstrated by discrepancies between model predictions and experimental observations for double pion production 49 , 50]. One reason for these discrepancies is the neglect of certain resonance contributions in the models. For example, reference [51] notes deficiencies in theoretical models that neglect the $\rho$ contribution in the second resonance region. Including the $\rho$ improved the agreement between these models and experimental results. However, neglecting or considering 
certain contributions is an oversimplification of the problem. Reference [50] studies the beam-helicity asymmetry for $\gamma p \rightarrow p \pi^{+} \pi^{-}$and shows that even at low energies, where few resonances contribute, experimental data disagree with model predictions. This is possibly due to the various non-resonant interactions that can occur between the final state particles.

In addition, the photoproduction of two pseudoscalar mesons off a nucleon target involves eight complex amplitudes, all of which are functions of five kinematic variables 17 and cannot be obtained from cross section measurements alone. In order to get a complete model of the reaction, 15 independent measurements are needed to obtain the contributing amplitudes and their phases 52. In addition, models like the ones in references 53 55], whose cross section predictions are accurate, fail at modeling polarization observables. This is due to a lack of understanding of the interactions involved so a better understanding of the reaction models is needed. One of the main theoretical difficulties in the modeling of reactions is that several subprocesses may contribute that, although small, interfere in nontrivial ways and have noticeable effects that can be measured. A better understanding of the reaction models along with polarization and cross section measurements may help in extracting and separating the individual contributing processes.

The lack of understanding of these reaction models has motivated many recent experiments and theory developments. A polarization observable that has recently drawn attention is the beam-helicity asymmetry. The beam-helicity asymmetry, $I^{\odot}$, arising from a circularly polarized photon beam, can be chosen to be an independent quantity yielding to a component of the eight complex amplitudes mentioned. This particular observable has previously been studied for double pion photoproduction in references $[49,50,52,56$ up to center-of-mass energy $W<2.3 \mathrm{GeV}$. The region with $W>2.3 \mathrm{GeV}$ was previously unexplored, but beam-helicity asymmetry measurements in this region are shown in this chapter. In addition, the first-time measurement for $I^{\odot}$ in the photoproduction of two charged kaons is also shown. Whereas the photoproduction of pions has been the subject of intense theoretical and experimental work, the kaon channel has until recently been largely neglected when compared to its nonstrange counterpart. The modeling of the kaon reaction also has a possible advantage over the pion reaction: because of the lack of evidence 
for experimentally confirmed $p K^{+}$resonances [18], there are fewer interfering production mechanisms for the kaon channel, and thus fewer expected parameters. This may prove fruitful for theorists working on reaction models as it is expected that the reduced number of final state interactions for the kaon channel will make it easier to analyze. However, the availability of kaon reaction models is limited. Currently, the only reaction model to consider two kaon production is in reference [57].

As shown in Fig. 4.1, many of the missing resonances lie in the $>2.0 \mathrm{GeV}$ region. However, these massive resonances are typically short-lived yielding broad, overlapping signals. Extracting these resonances from cross section measurements alone is an unrealistic approach. Also, while invariant mass distributions convey important information on the reaction mechanisms, they are not the most sensitive of observables and do not provide a complete test of the quark models provided. This work explores the beam-helicity asymmetry for the photoproduction of two charged kaons, which had not been measured before. This work also explores the sensitivity of $I^{\odot}$ to $W$ up to $3.3 \mathrm{GeV}$ for both the $\gamma p \rightarrow p \pi^{+} \pi^{-}$ and $\gamma p \rightarrow p K^{+} K^{-}$reactions. In addition, $I^{\odot}$ for $\gamma p \rightarrow p K^{+} K^{-}$is studied as a function of several kinematic variables that are key in modeling the reaction dynamics. These variables include the momentum transfers from the photon to the $K^{+}$and the $K^{+} K^{-}$system, and invariant masses of the $K^{+} K^{-}, p K^{-}$, and $p K^{+}$systems. 

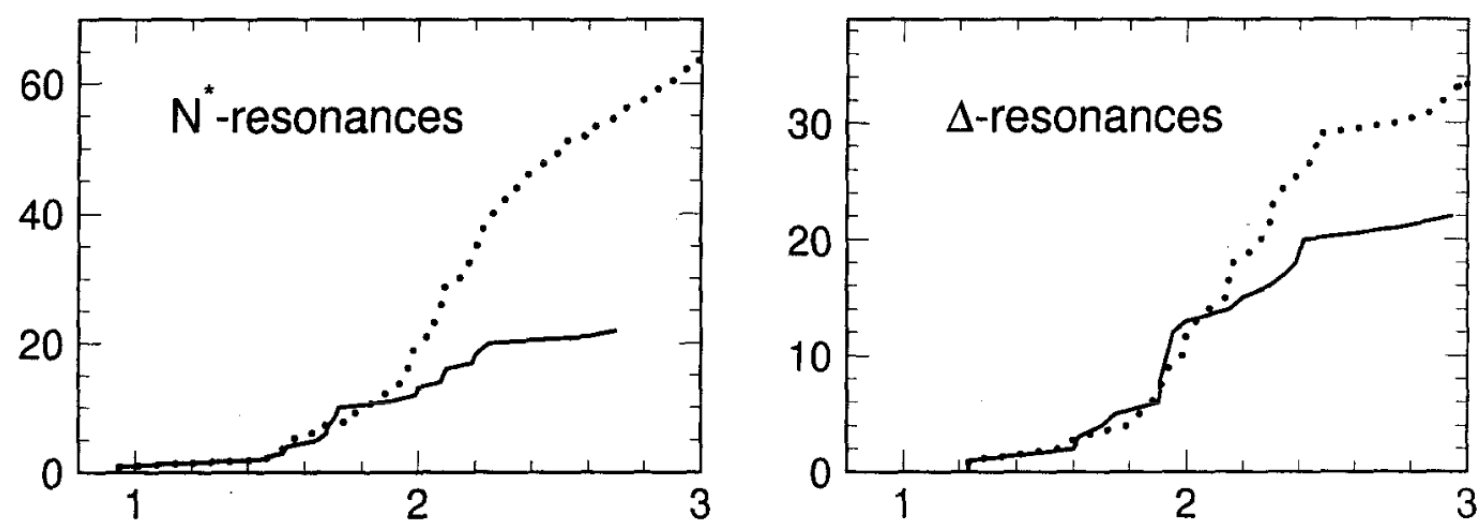

$\mathrm{W}[\mathrm{GeV}]$

Figure 4.1: Comparison of number of predicted and observed states as a function of resonance mass. Dotted line is the number of predicted states. Solid line is the number of observed states. Source: 11

\subsection{Preliminaries}

In a given kinematic bin, $\tau$, the beam-helicity asymmetry is defined as

$$
I^{\odot}(\tau)=\frac{\sigma^{+}(\tau)-\sigma^{-}(\tau)}{\sigma^{+}(\tau)+\sigma^{-}(\tau)},
$$

where $\sigma$ is the total cross section, the superscripts \pm refer to an event in which the photon was in a \pm helicity state, and $P_{\gamma}$ is the polarization of the photon. The asymmetry arises from the different spin orientations of the photon beam and interference of different production processes. It is measured experimentally as

$$
I_{\exp }^{\odot}(\tau)=\frac{\frac{Y^{+}(\tau)}{\alpha^{+}}-\frac{Y^{-}(\tau)}{\alpha^{-}}}{\frac{N^{+}(\tau)}{\alpha^{+}}+\frac{N^{-}(\tau)}{\alpha^{-}}} .
$$


The factors $\alpha^{ \pm}$take into account the primary electron beam charge asymmetry between the two helicity states and are given by

$$
\begin{aligned}
\alpha^{ \pm} & =\frac{1}{2}\left(1 \pm \overline{a_{c}}\right), \text { where } \\
\bar{a}_{c} & =\frac{N_{\pi}^{+}-N_{\pi}^{-}}{N_{\pi}^{+}+N_{\pi}^{-}}=0.0028 \pm 0.0008
\end{aligned}
$$

The beam charge asymmetry was measured by analyzing the reactions $\gamma p \rightarrow p \pi^{0}$ and $\gamma p \rightarrow n \pi^{+}$and is discussed in Section 4.2. The photon polarization is transferred from the electron beam according to the Maximon-Olsen equation [28]:

$$
P_{\gamma}=\frac{E_{\gamma}\left(E_{e}+\frac{E_{e}-E_{\gamma}}{3}\right)}{E_{e}^{2}+\left(E_{e}-E_{\gamma}\right)^{2}-\frac{2}{3} E_{e}\left(E_{e}-E_{\gamma}\right)} P_{e}
$$

where $E_{\gamma}$ and $E_{e}$ are the photon and electron beam energies, respectively, and $P_{e}$ is the electron beam polarization. Its graph is shown in Fig. 4.2. The electron beam polarization was measured using a Møller polarimeter several times over the course of the experiment and its measurements are shown in Table 4.1. The yields $Y^{ \pm}(\tau)$ are the sums of the polarization-weighted events:

$$
Y^{ \pm}(\tau)=\sum_{i=1}^{N^{ \pm}(\tau)} \frac{1}{P_{\gamma, i}^{ \pm}}
$$

where \pm superscripts denotes a \pm helicity state for an event, $P_{\gamma, i}^{ \pm}$is the photon polarization for the $i^{\text {th }}$ event in $\tau$, and $N^{ \pm}(\tau)$ denotes the number of events in $\tau$ coming from a \pm helicity state.

The beam-helicity asymmetry can be studied as a function of many variables. In this study, the beam-helicity asymmetry's angular dependence on center-of-mass energy, invariant masses, and momentum transfers is studied. The angle of interest is defined as the azimuthal angle using the following configurations: given a five-body system in the centerof-mass frame (net momentum is zero), there are three different plane-angle configurations that can be chosen. The configurations are defined in the following way: 


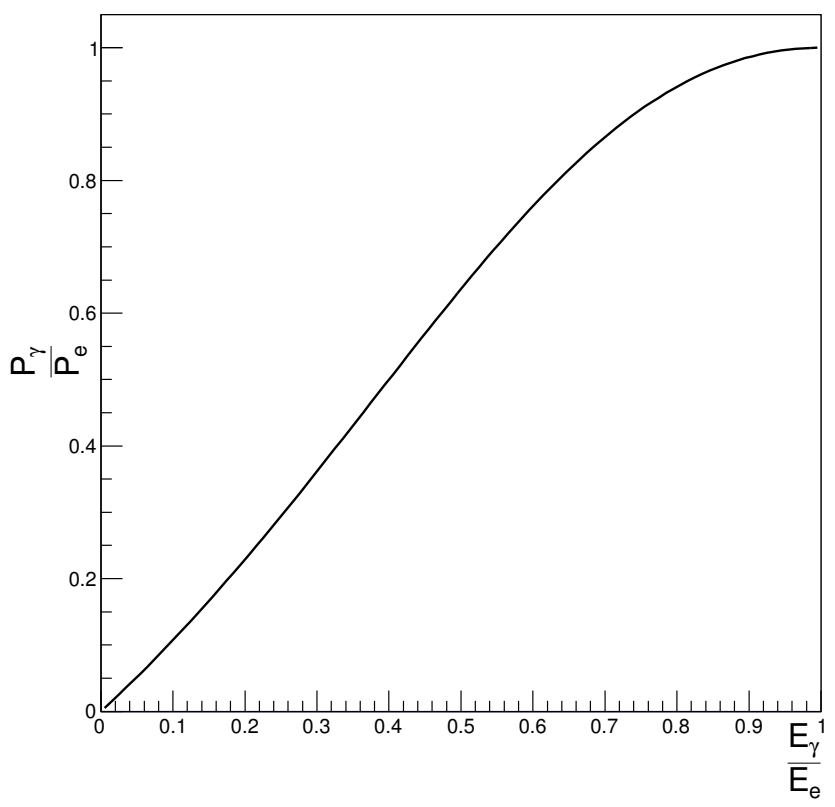

Figure 4.2: Maximon-Olsen equation. $P_{e}$ is the polarization of the incident electron beam, $P_{\gamma}$ is the polarization of the outgoing photon beam, $E_{e}$ is the energy of the incident electron beam, and $E_{\gamma}$ is the energy of the outgoing photon beam.

- Meson-Meson Plane Configuration: The meson-meson configuration (Section 4.3 is defined so that the $z$ axis is parallel to the meson-meson system, $\hat{y}=\hat{\gamma} \times \hat{z}$, and $\hat{x}=\hat{y} \times \hat{z}$, as shown in Fig. $4.3 \mathrm{a}$.

- Neutral Baryon Plane Configuration: The neutral baryon configuration (Section 4.4 is defined so that the $z$ axis is parallel to the proton-negative meson system with $\hat{y}$ and $\hat{x}$ defined as above, as shown in Fig. $4.3 \mathrm{~b}$

- Positive Baryon Plane Configuration: The positive baryon configuration (Section 4.5 is defined so that the $z$ axis is parallel to the proton-positive meson system with $\hat{y}$ and $\hat{x}$ defined as above, as shown in Fig. $4.3 \mathrm{c}$

Since the beam-helicity asymmetry is sensitive to interfering production mechanisms and the angle between two predefined planes, it must also depend on the different plane and angle definitions. The differing features in the beam-helicity asymmetry with respect to the different plane definitions may be due to production mechanisms interfering differently. Studying the beam-helicity asymmetry with different plane definitions can then be used to 
probe the contribution of different production mechanisms. The beam-helicity asymmetry for each configuration is shown in Sections 4.3 to 4.5 .

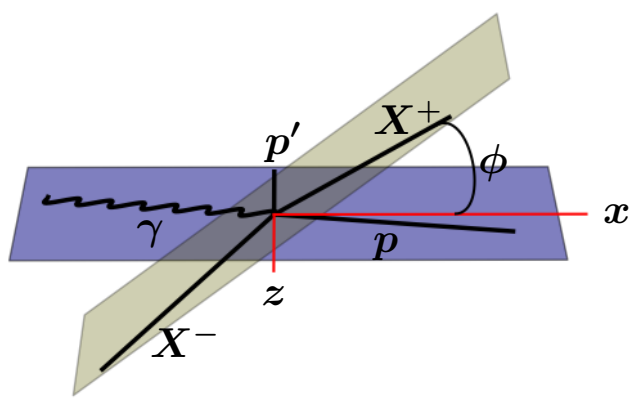

(a) Meson-meson configuration

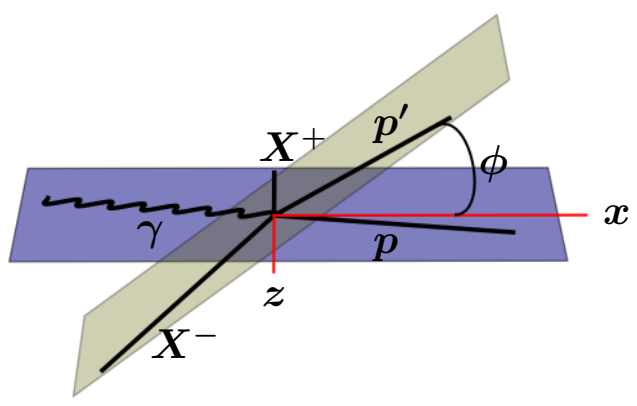

(b) Neutral baryon configuration

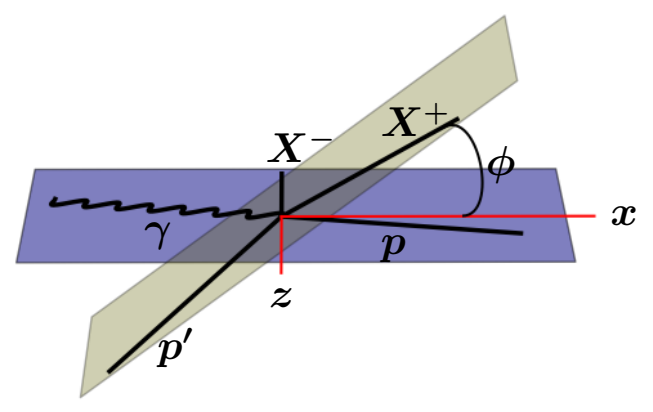

(c) Positive baryon configuration

Figure 4.3: Plane-angle configurations. 


\begin{tabular}{|l|c|}
\hline Run Range & Møller Readout $\left(P_{e}\right)$ \\
\hline $56355-56475$ & $(81.221 \pm 1.48) \%$ \\
$56476-56643$ & $(67.166 \pm 1.21) \%$ \\
$56644-56732$ & $(59.294 \pm 1.47) \%$ \\
$56733-56743$ & $(62.071 \pm 1.46) \%$ \\
$56744-56849$ & $(62.780 \pm 1.25) \%$ \\
$56850-56929$ & $(46.490 \pm 1.47) \%$ \\
$56930-57028$ & $(45.450 \pm 1.45) \%$ \\
$57029-57177$ & $(68.741 \pm 1.38) \%$ \\
$57178-57249$ & $(70.504 \pm 1.46) \%$ \\
$57250-57282$ & $(75.691 \pm 1.46) \%$ \\
$57283-57316$ & $(68.535 \pm 1.44) \%$ \\
\hline
\end{tabular}

Table 4.1: The degree of longitudinal electron polarization $\left(P_{e}\right)$ for each Møller run. The uncertainties shown are statistical uncertainties. The systematic uncertainty is estimated to be a relative $5 \%$. 


\subsection{Beam Charge Asymmetry}

The incident electron beam from CEBAF was longitudinally polarized and transferred its polarization to the photon beam. The relation between the electron and photon polarization is given by Eq. 4.5. For the g12 experiment, the electron-beam helicity was flipped at a rate of $30 \mathrm{~Hz}$. Certain instrumental asymmetries may propagate into the beam-helicity asymmetry results and must be corrected.

The beam-charge asymmetry, shown in Eqs. 4.3 and 4.4 , was measured by analyzing the reactions $\gamma p \rightarrow p \pi^{0}$ and $\gamma p \rightarrow n \pi^{+}$obtained from g12's single sector runs. As the beam-helicity asymmetry for two-body parity conserving reactions is identically zero, the asymmetry measured from these reactions is purely instrumental. As the neutral particles were not required to be detected, they were reconstructed from missing mass and momentum using kinematic fitting. The beam-charge asymmetry was then defined by Eq. 4.4 where $N_{\pi}^{ \pm}$is the total number of pions detected from a \pm beam-helicity state. The beam-charge asymmetry was measured to be 0.0028 with statistical uncertainty 0.0008 ( $c f$. Section 4.6).

\subsection{Meson-Meson Plane Configuration}

Figure 4.4 shows the angular dependence of the beam-helicity asymmetry for two charged kaon and two charged pion photoproduction in the meson-meson plane configuration shown in Fig. 4.3a. In this coordinate system, the azimuthal angle $\phi$ measures the angle between the planes defined by the two-meson system and the production plane. Due to the sinusoidal nature of the asymmetry, fitting the asymmetry to a truncated Fourier sine series,

$$
I^{\odot}(\phi ; \tau)=\sum_{n=1}^{3} c_{n}(\tau) \sin (n \phi),
$$

is beneficial to study its dependence on other kinematic variables, say $\tau$, by analyzing the behavior of the coefficients, $c_{n}(\tau)$. This fit is applied to both the pion and kaon asymmetries, and is shown in Fig. 4.4. The Fourier series was determined to be truncated after three 
coefficients after several significance tests, such as hypothesis testing, resulted that the other coefficients were statistically consistent with zero for the fits shown in Fig. 4.4.

The pion and kaon channels have two significant differences: First, the kaon asymmetry is dominated by $\sin (\phi)$ while the pion channel is dominated by $\sin (2 \phi)$. Second, the overall amplitude for the kaon asymmetry is significantly larger than the pion asymmetry. This suggests different production mechanisms between the strange and nonstrange channels. The results shown in Fig. 4.4 are binned only in $\phi$ and summed over all other kinematics.

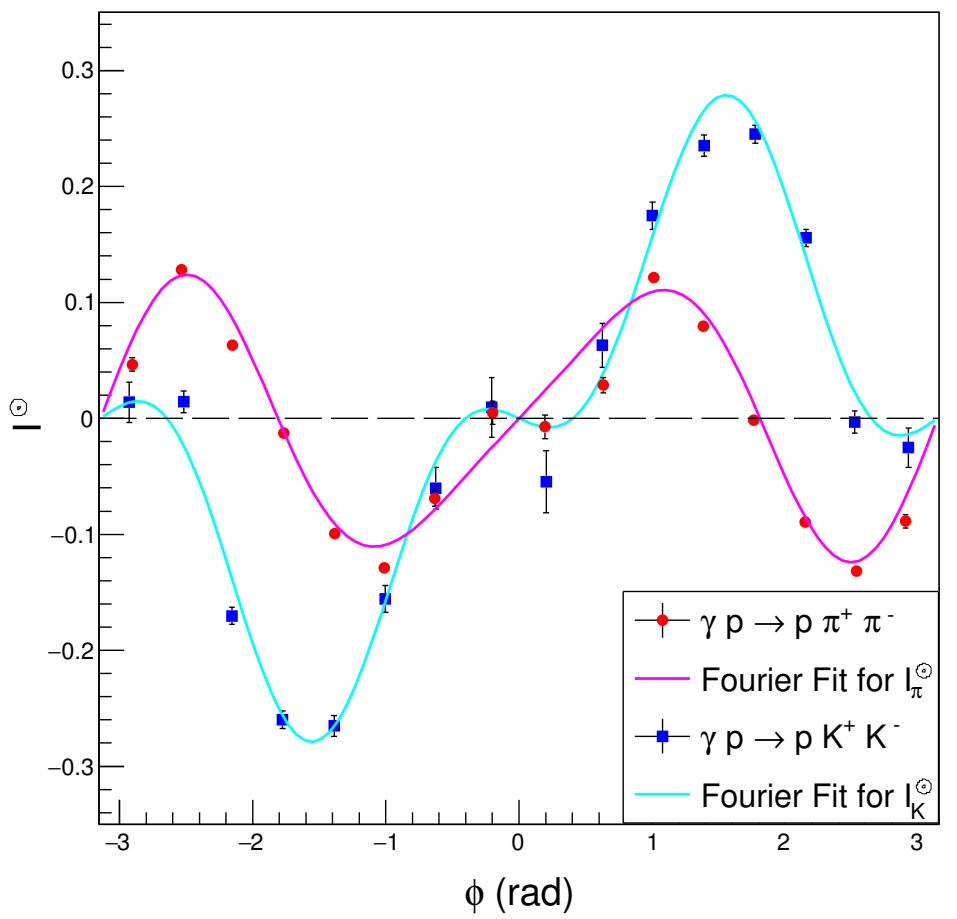

Figure 4.4: The angular dependence in the meson-meson configuration of the beam-helicity asymmetry for double-charged-pion and kaon photoproduction summed over $E_{\gamma}>1.1 \mathrm{GeV}$, momentum transfers, and invariant masses.

The angular dependence of the beam-helicity asymmetry was measured while binning with respect to several kinematic variables, and fitted to a third order Fourier sine series. One kinematic variable of importance is the overall center-of-mass energy, $W$. Figure 4.5 shows the dependence of the Fourier coefficients as a function of $W$. The fits for the kaon 
reaction shows that it is $\sin (\phi)$ dominated $\left(\left|c_{1}\right|>\left|c_{2}\right|,\left|c_{3}\right|\right)$ while the fits for the pion reaction shows $\sin (2 \phi)$ dominance $\left(\left|c_{2}\right|>\left|c_{1}\right|,\left|c_{3}\right|\right)$ for most of the energy range.

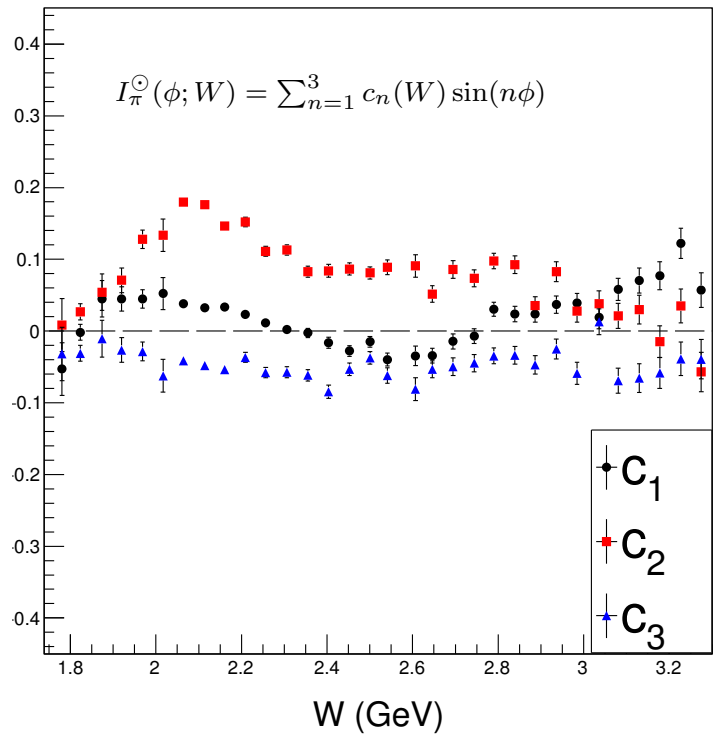

(a) Pion $I^{\odot}$ Fourier coefficients as function of $W$

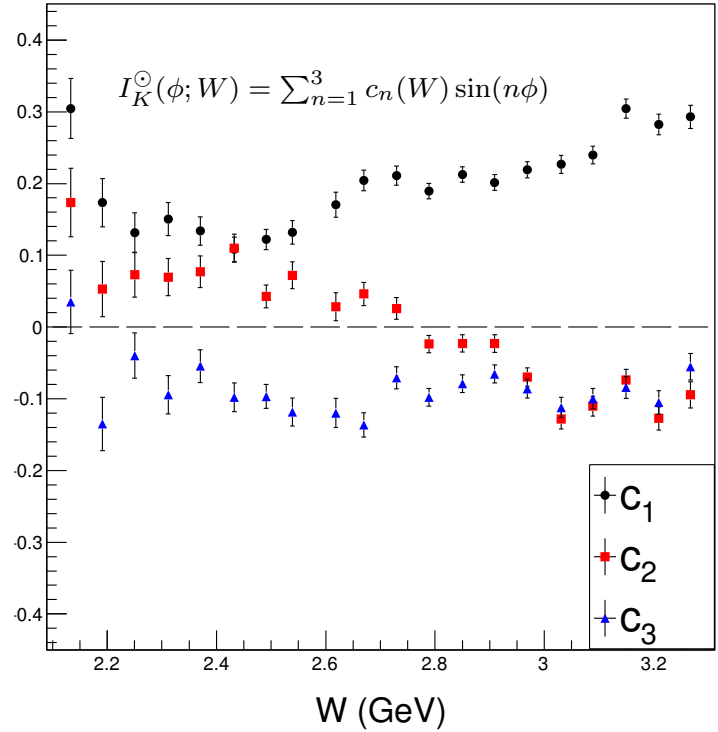

(b) Kaon $I^{\odot}$ Fourier coefficients as function of $W$

Figure 4.5: Fourier coefficients as function of center-of-mass energy in the meson-meson configuration, summed over invariant masses and momentum transfers.

$I^{\odot}$ for the kaon reaction was also measured as a function of the $K^{+} K^{-}, p K^{-}$, and $p K^{+}$invariant masses as shown in Fig. 4.6. A feature of these data is that the dominant term $c_{1}$ of the asymmetry diminishes as the invariant mass of $K^{+} K^{-}$increases, whereas it increases as the invariant mass of either $p K^{-}$or $p K^{+}$increases. In addition, as a function of $M\left(K^{+} K^{-}\right), I^{\odot}$ has a local maximum at $M\left(K^{+} K^{-}\right) \approx 1.1 \mathrm{GeV}$. A similar behavior occurs as a function of $M\left(p K^{+}\right)$: a local maximum occurs at $M\left(p K^{+}\right) \approx 1.5 \mathrm{GeV}$.

Another feature of the asymmetry is its behavior with respect to the momentum transfers to the $K^{+}$and $K^{+} K^{-}$systems as shown in Fig. 4.7. The asymmetry as a function of $t_{\gamma \rightarrow K^{+}}$shows a smoothly increasing dominant term $c_{1}$ reaching a maximum, followed by a smooth decrease towards zero at large momentum transfers. The asymmetry is also shown to be dominated by the $\sin (\phi)$ term throughout the $t_{\gamma \rightarrow K^{+}}$range. The asymmetry as function of $t_{\gamma \rightarrow K^{+} K^{-}}$shows a more complicated behavior, with the coefficients achieving 


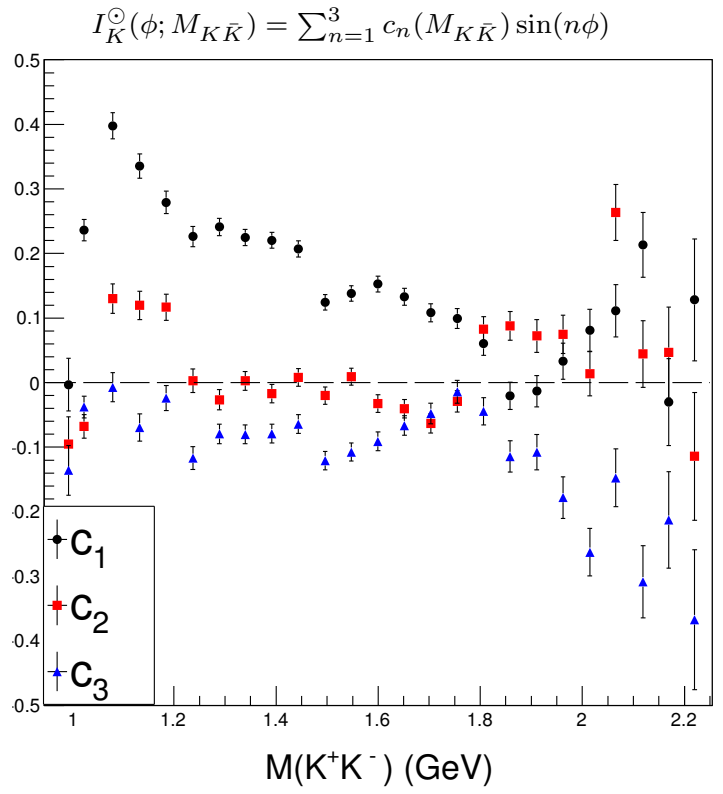

(a) Kaon $I^{\odot}$ Fourier coefficients as function of $M\left(K^{+} K^{-}\right)$

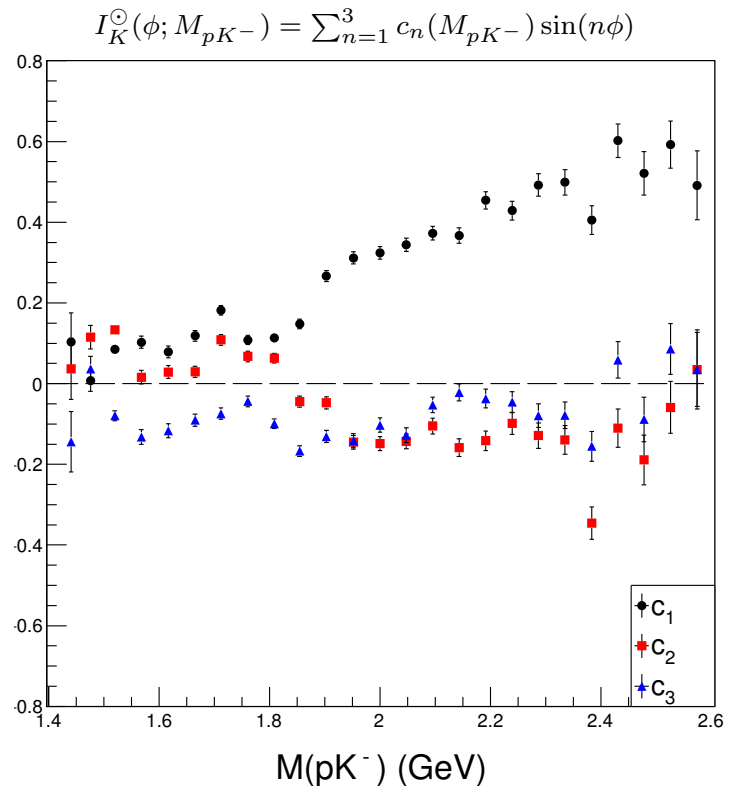

(b) Kaon $I^{\odot}$ Fourier coefficients as function of $M\left(p K^{-}\right)$

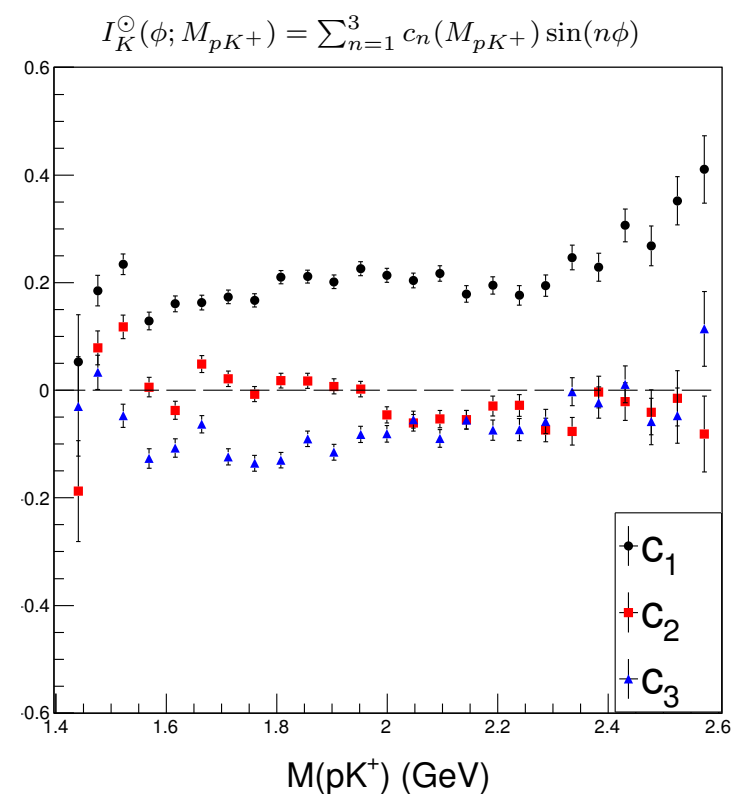

(c) Kaon $I^{\odot}$ Fourier coefficients as function of $M\left(p K^{+}\right)$

Figure 4.6: Fourier coefficients as function of invariant masses, summed over $E_{\gamma}>1.1 \mathrm{GeV}$ and momentum tranfers. 
a local maximum or minimum at different $t_{\gamma \rightarrow K^{+} K^{-}}$. However, it maintains roughly the same behavior as a function of $t_{\gamma \rightarrow K^{+}}$: the coefficients reach a local maximum/minimum and then vanish at large momentum transfers.

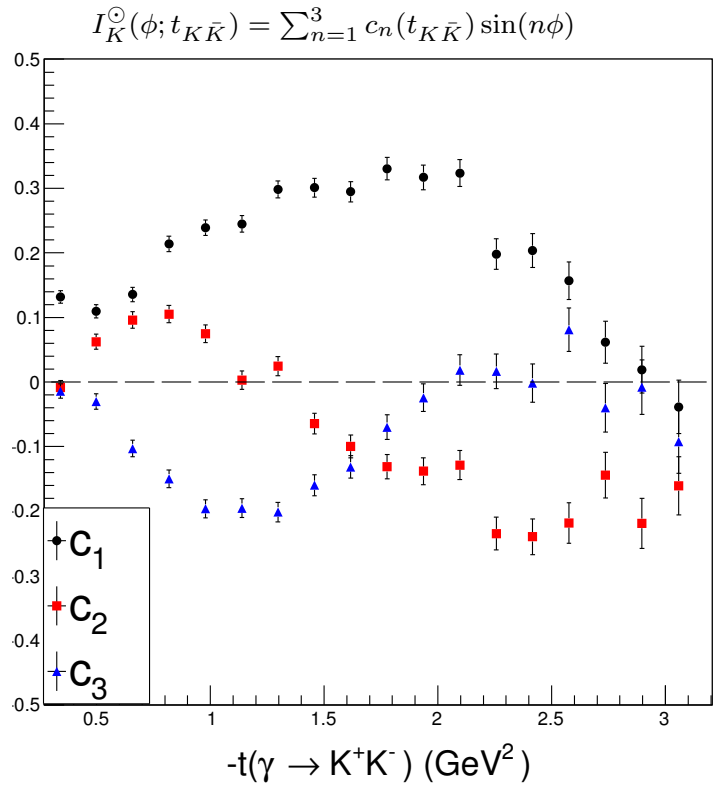

(a) Kaon $I^{\odot}$ Fourier coefficients as function of $t_{\gamma \rightarrow K^{+} K^{-}}$

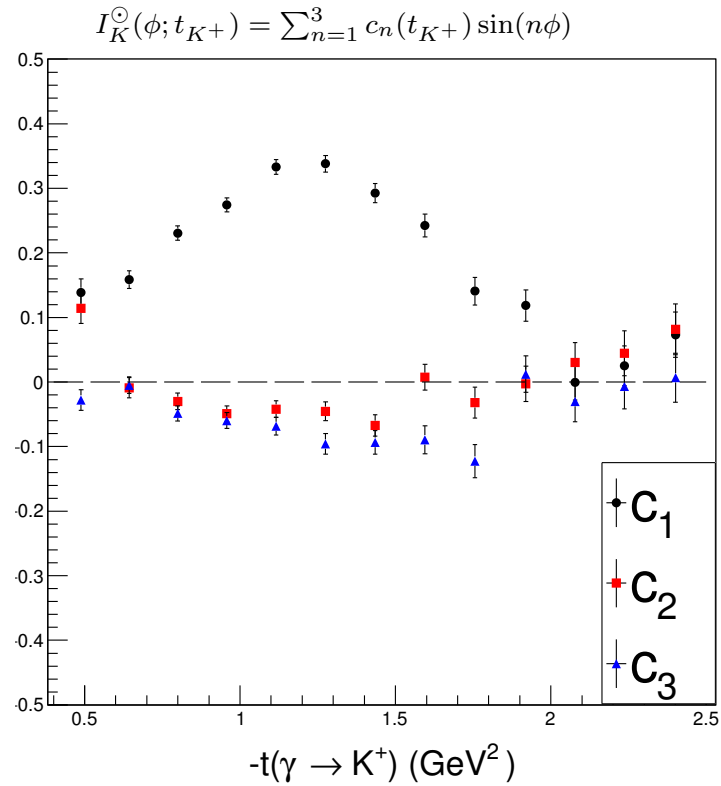

(b) Kaon $I^{\odot}$ Fourier coefficients as function of $t_{\gamma \rightarrow K^{+}}$

Figure 4.7: Fourier coefficients as function of momentum transfer.

\subsection{Neutral Baryon Configuration}

Figure 4.8 shows the angular dependence of the kaon asymmetry along with its fit to Eq. 4.7 when the $z$ axis is chosen to be parallel to the $p K^{-}$system, designated the neutral baryon configuration and shown in Fig. 4.3b. In this configuration, the asymmetry is also dominated by the $\sin (\phi)$ term. The Fourier coefficients as a function of $W$, shown in Fig. 4.9, show that main contribution is coming from the $\sin (\phi)$ term, which increases as the energy increases. The $\sin (2 \phi)$ and $\sin (3 \phi)$ contributions are roughly constant throughout the energy range.

As a function of the invariant masses (Fig. 4.11), the asymmetry in this plane-angle configuration shows roughly the same behavior as in the meson-meson configuration, but 


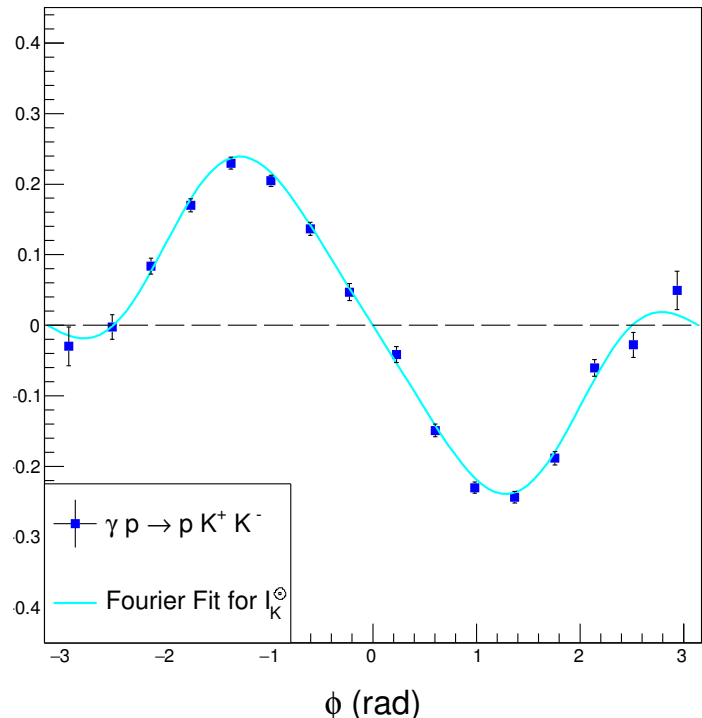

Figure 4.8: The angular dependence in the neutral baryon configuration of the beamhelicity asymmetry for double-charged-kaon photoproduction summed over $E_{\gamma}>1.1$ $\mathrm{GeV}, \cos \left(\theta_{\mathrm{cm}}\right)$, and invariant masses.

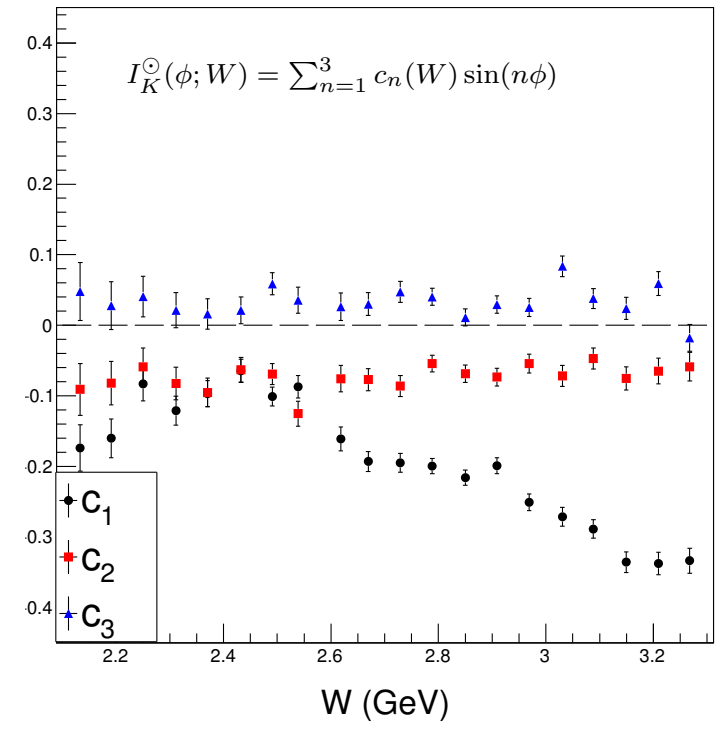

Figure 4.9: Fourier coefficients as function of center-of-mass energy. 
with an overall multiplicative factor of -1 : As a function of $M\left(K^{+} K^{-}\right)$the dominant term, $c_{1}$ reaches a local minimum at $M\left(K^{+} K^{-}\right) \approx 1.1 \mathrm{GeV}$ followed by a trend towards zero as $M\left(K^{+} K^{-}\right)$increases. As functions of the $p K^{-}$or $p K^{+}$invariant masses, the $c_{1}$ term increases in magnitude as the invariant masses increase.

Finally, as a function of momentum transfers (Fig. 4.10), the asymmetry in this configuration demonstrates qualitatively the same behavior as the meson-meson configuration. As a function of $t_{\gamma \rightarrow K^{+}}$, the overall amplitude increases as the momentum transfer increases until it reaches a maximum and decreases to zero at large momentum transfers. As a function of $t_{\gamma \rightarrow K^{+} K^{-}}$, the asymmetry follows a more complicated behavior. An interesting feature of the asymmetry as a function $t_{\gamma \rightarrow K^{+} K^{-}}$is that its coefficients appear to change their overall signs at large $-t_{\gamma \rightarrow K^{+} K^{-}}$.

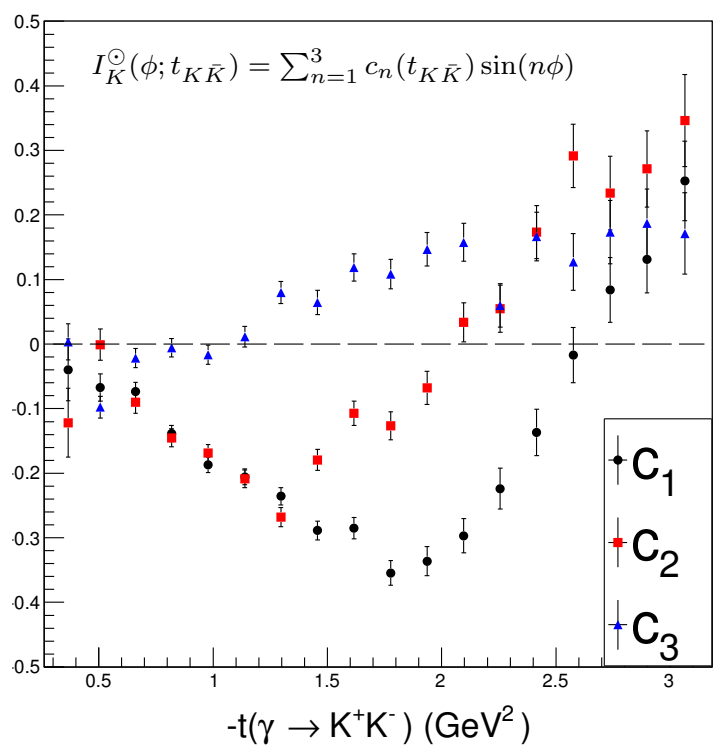

(a) Kaon $I^{\odot}$ Fourier coefficients as function of $t_{\gamma \rightarrow K^{+} K^{-}}$

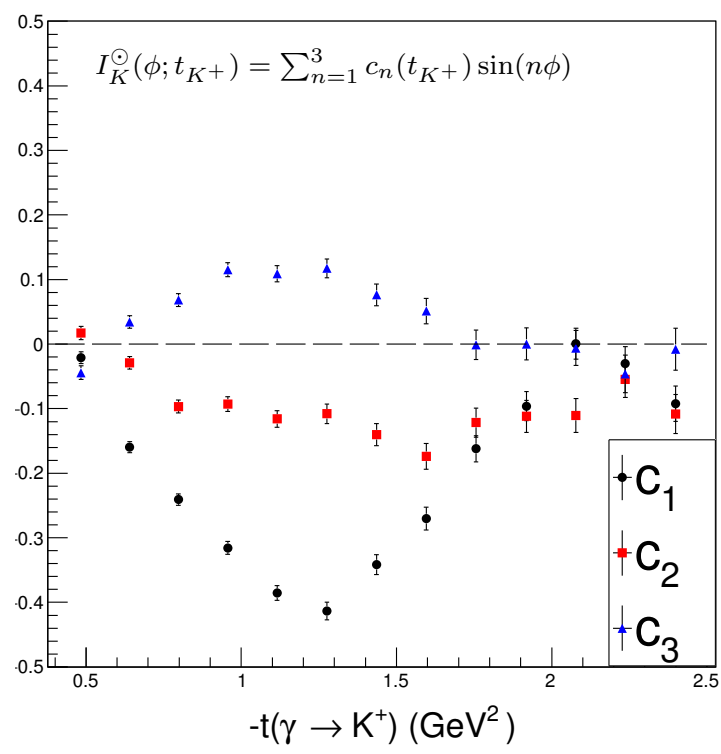

(b) Kaon $I^{\odot}$ Fourier coefficients as function of $t_{\gamma \rightarrow K^{+}}$

Figure 4.10: Fourier coefficients as function of momentum transfer. 


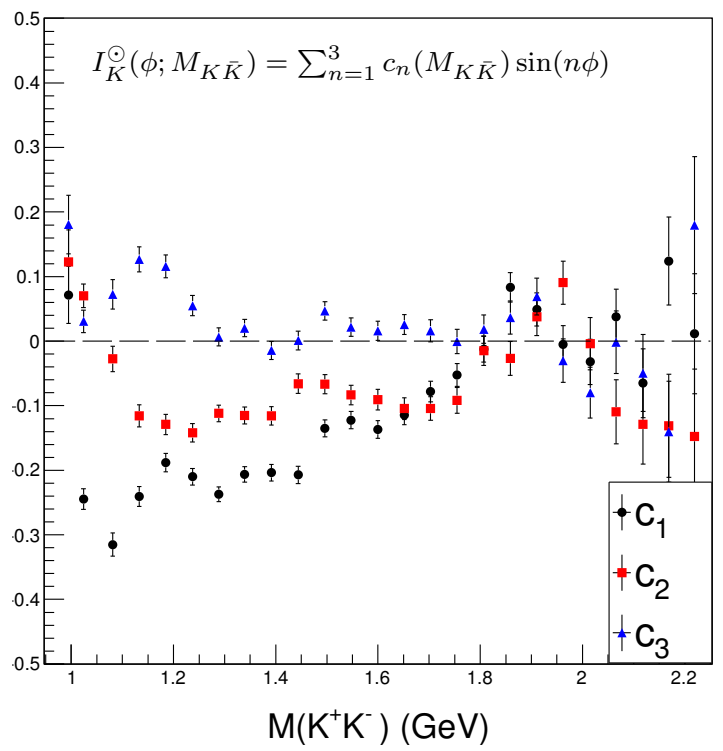

(a) Kaon $I^{\odot}$ Fourier coefficients as function of $M\left(K^{+} K^{-}\right)$

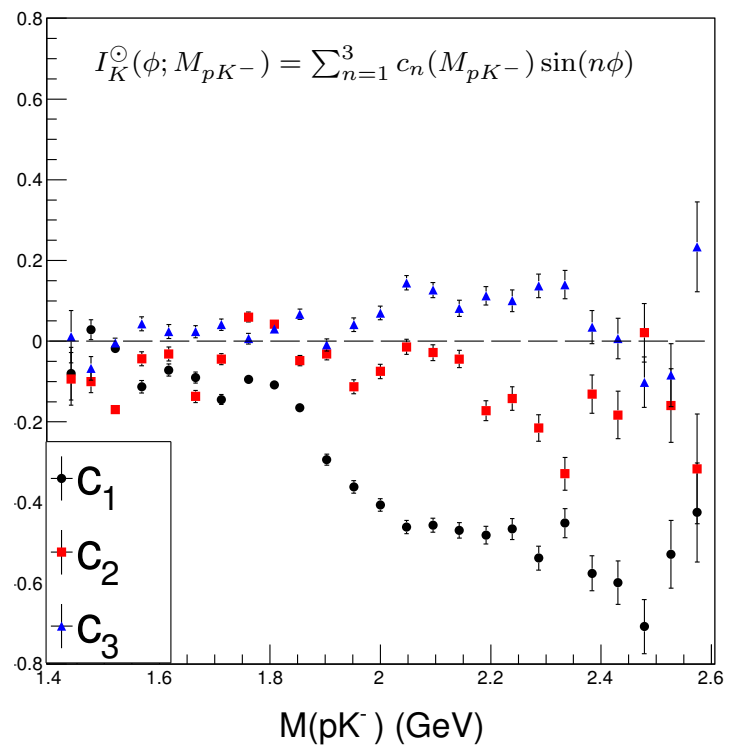

(b) Kaon $I^{\odot}$ Fourier coefficients as function of $M\left(p K^{-}\right)$

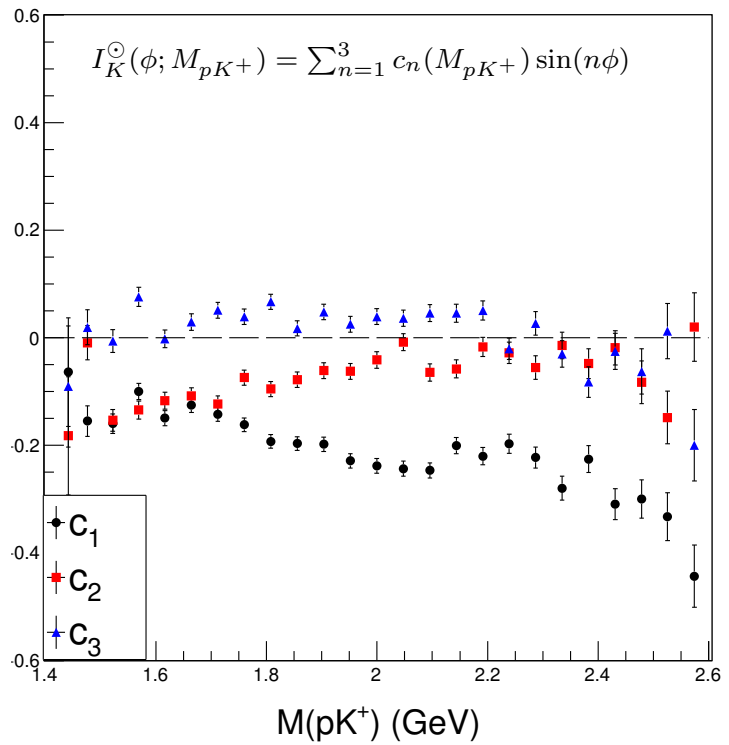

(c) Kaon $I^{\odot}$ Fourier coefficients as function of $M\left(p K^{+}\right)$

Figure 4.11: Fourier coefficients as function of invariant masses. 


\subsection{Positive Baryon Configuration}

The other possible configuration is with the $z$ axis is parallel to the $p K^{+}$system and shown in Fig. 4.3b Figure 4.12 shows the asymmetry in this configuration. Like the other two plane-angle configurations, it dominated by the $\sin (\phi)$ term. However, unlike the other two configurations, the asymmetry in this configuration has a significant $\sin (2 \phi)$ contribution as shown by decomposing into its Fourier coefficients. Figure 4.13 shows a large $\sin (2 \phi)$ contribution, which gets larger as $W$ increases. The $\sin (3 \phi)$ term also has a region in $W$ for which it contributes significantly.

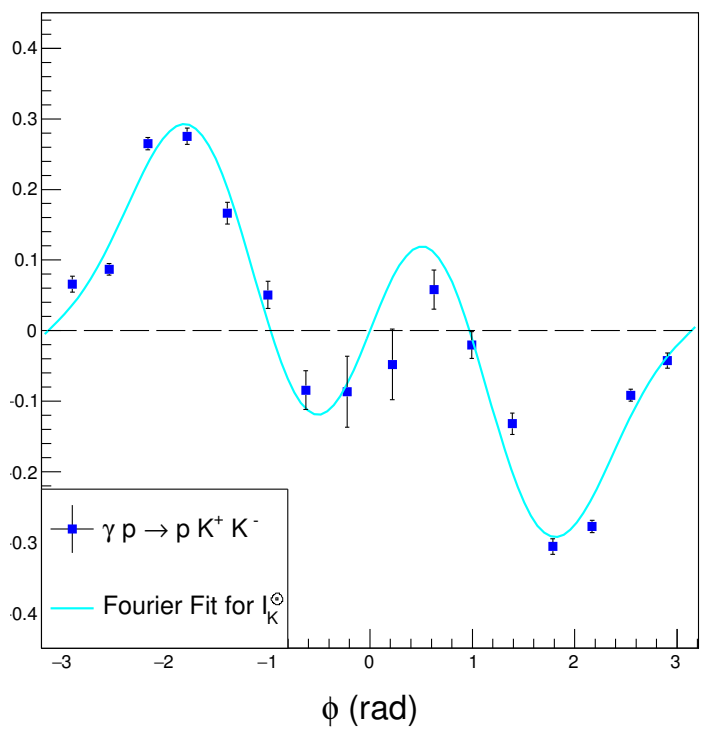

Figure 4.12: The angular dependence in the neutral baryon configuration of the beamhelicity asymmetry for double-charged-kaon photoproduction summed over $E_{\gamma}>1.1$ $\mathrm{GeV}, \cos \left(\theta_{\mathrm{cm}}\right)$, and invariant masses.

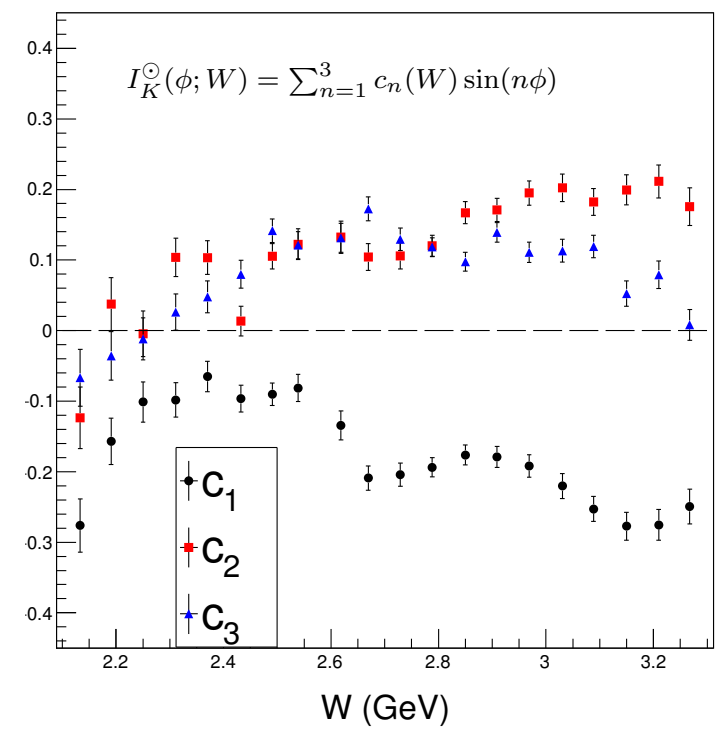

Figure 4.13: Fourier coefficients as function of center-of-mass energy.

As a function of the invariant masses (Fig. 4.15), the asymmetry appears to follow the same qualitative pattern as the other two configurations: the asymmetry decreases in magnitude as the invariant mass of $K^{+} K^{-}$increases. It also increases as the $p K^{-}$and $p K^{+}$ 
invariant masses increase as shown in Fig. 4.15. A point of interest is once again the local minimum at $M\left(K^{+} K^{-}\right) \approx 1.1 \mathrm{GeV}$ that is seen in the neutral baryon and meson-meson configuration $s^{1}$

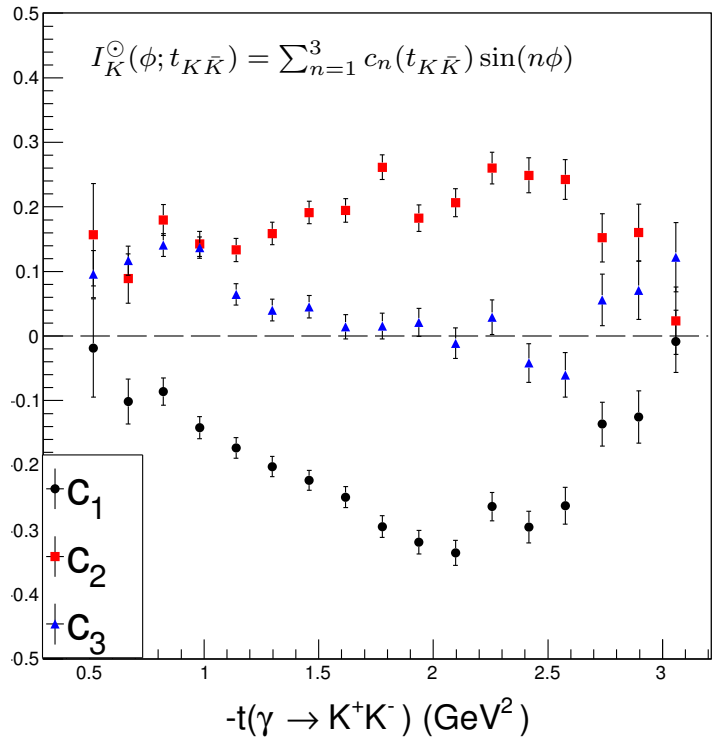

(a) Kaon $I^{\odot}$ Fourier coefficients as function of $t_{\gamma \rightarrow K^{+} K^{-}}$

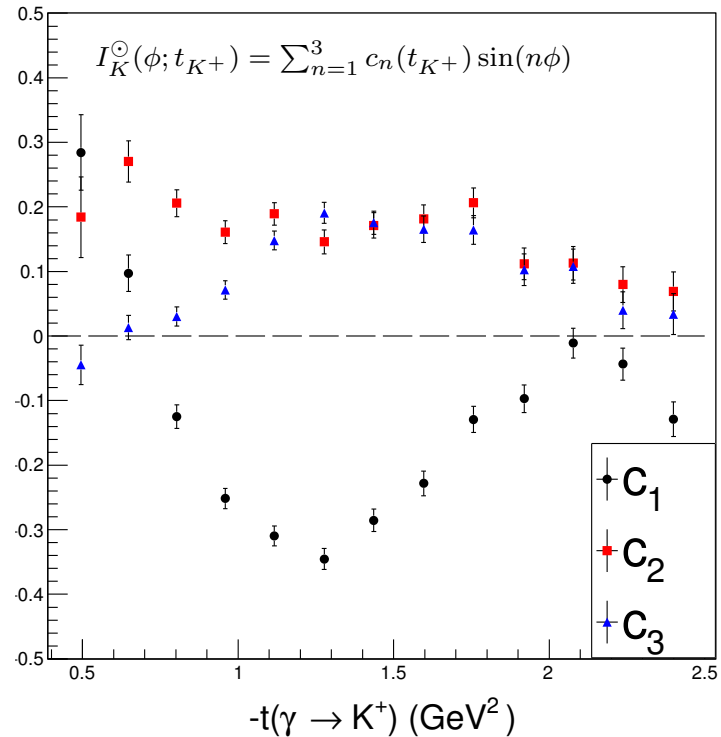

(b) Kaon $I^{\odot}$ Fourier coefficients as function of $t_{\gamma \rightarrow K^{+}}$

Figure 4.14: Fourier coefficients as function of momentum transfer.

Finally, the asymmetry's coefficients as a function of $t_{\gamma \rightarrow K^{+}}$and $t_{\gamma \rightarrow K^{+} K^{-}}$is shown for this plane-angle configuration in Fig. 4.14. As a function of $t_{\gamma \rightarrow K^{+}}$, the asymmetry's $c_{1}$ coefficient decreases until it reaches a local minimum and appears to vanish at large momentum transfers. Unlike the previous two configurations, the positive baryon configuration for kaons shows a significant $\sin (2 \phi)$ contribution throughout a large kinematic region. As a function of $t_{\gamma \rightarrow K^{+} K^{-}}$, a significant $\sin (2 \phi)$ contribution is also shown. At large $t_{\gamma \rightarrow K^{+}}$and $t_{\gamma \rightarrow K^{+} K^{-}}$, the asymmetry's magnitude appears to decrease to zero.

\footnotetext{
${ }^{1}$ Local maximum in the meson-meson configuration
} 


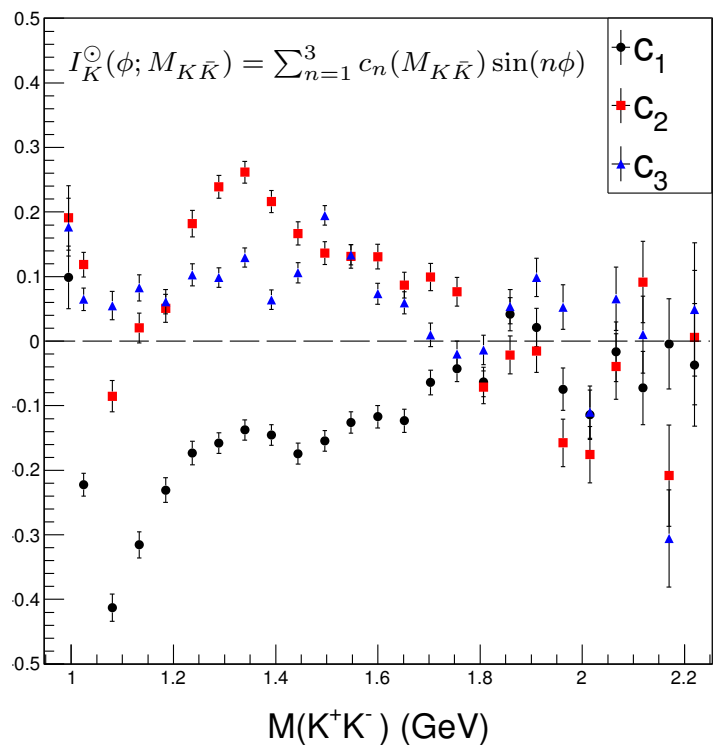

(a) Kaon $I^{\odot}$ Fourier coefficients as function of $M\left(K^{+} K^{-}\right)$

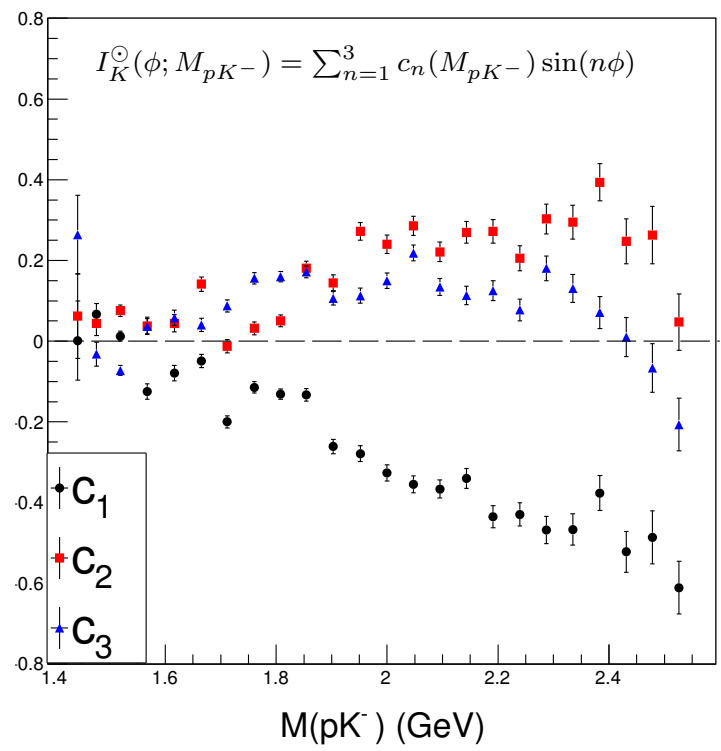

(b) Kaon $I^{\odot}$ Fourier coefficients as function of $M\left(p K^{-}\right)$

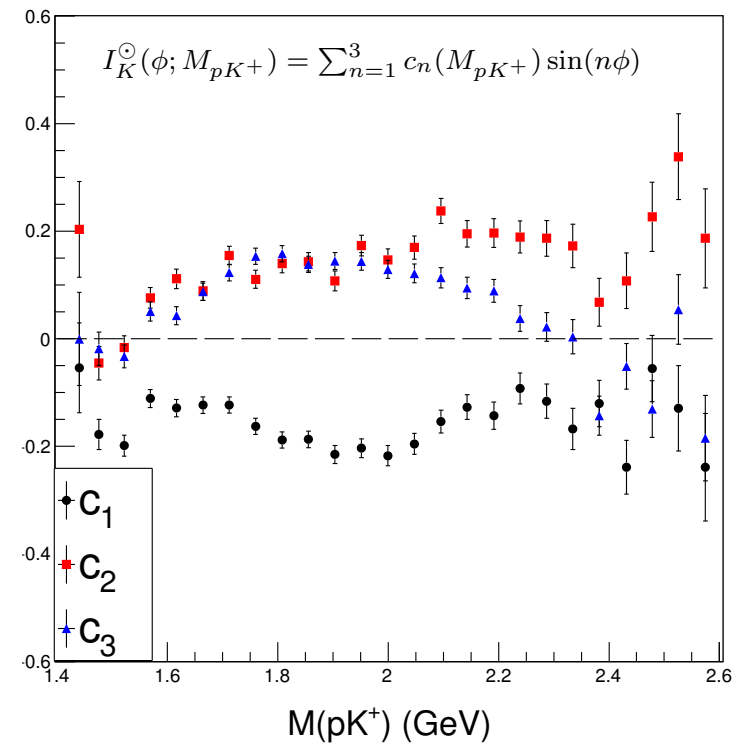

(c) Kaon $I^{\odot}$ Fourier coefficients as function of $M\left(p K^{+}\right)$

Figure 4.15: Fourier coefficients as function of invariant masses. 


\subsection{Statistical Uncertainties}

The error bars shown in Figs. 4.4, 4.8 and 4.12 are the statistical uncertainties, pri-

marily from "counting." The error bars shown for the Fourier coefficients shown in Figs. 4.5 to 4.7 . 4.9 to 4.11 and 4.13 to 4.15 are parameter uncertainties from performing a least chi-squared fit to Eq. 4.7.

Let $\tau$ be a given kinematic bin. The beam-helicity asymmetry in $\tau$ is measured following Eq. 4.2. Note that for every event in $\tau$, an event can only contribute \pm 1 depending on its helicity state before being weighted by the photon polarization. Consider the Bernoulli-type distribution

$$
Z \sim\left\{\begin{array}{c}
f(-1)=q \\
f(1)=p
\end{array}\right.
$$

where $p, q>0$ and $p+q=1$. Define the random variable $S$

$$
S(p ; q)=\frac{1}{N} \sum_{i=1}^{N} \frac{Z_{i}}{P_{i}}
$$

where the $P_{i}$ are given. Denote $\mathbb{E}(S)$ to be the expectation value of $S$ :

$$
\mathbb{E}(S)=\mathbb{E}\left(\frac{1}{N} \sum_{i=1}^{N} \frac{Z_{i}}{P_{i}}\right)
$$

Using the linearity property of $\mathbb{E}$, it follows that

$$
\mathbb{E}(S)=\frac{1}{N} \mathbb{E}\left(\sum_{i=1}^{N} \frac{Z_{i}}{P_{i}}\right) .
$$

The expectation value of the sum can be computed: since $Z_{i}$ can only attain values \pm 1 , the expectation value of the sum is the difference between the expected number of times a value of +1 is drawn and the expected number of times a value of -1 is drawn, and weighted by 
$1 / P_{i}$ for the $i^{\text {th }}$ instance, i.e.

$$
\mathbb{E}(S)=\frac{1}{N}\left(\sum_{i_{+}=1}^{\mathbb{E}\left(\# Z_{i}=1\right)} \frac{1}{P_{i_{+}}}-\sum_{i_{-}=1}^{\mathbb{E}\left(\# Z_{i}=-1\right)} \frac{1}{P_{i_{-}}}\right)
$$

The expected number of times a value of +1 is drawn is $N p$. Likewise, the expected number of times a value of -1 is drawn is $N q$. Hence,

$$
\mathbb{E}(S)=\frac{1}{N}\left(\sum_{i_{+}=1}^{N p} \frac{1}{P_{i_{+}}}-\sum_{i_{-}=1}^{N q} \frac{1}{P_{i_{-}}}\right)
$$

The variance of $S$, denoted $\operatorname{Var}(S)$, is also computed. To start, the variance of $S$ is given by

$$
\operatorname{Var}(S)=\operatorname{Var}\left(\frac{1}{N} \sum_{i=1}^{N} \frac{Z_{i}}{P_{i}}\right)
$$

Following the property of variances under linear transformations,

$$
\begin{aligned}
\operatorname{Var}(S) & =\frac{1}{N^{2}} \operatorname{Var}\left(\sum_{i=1}^{N} \frac{Z_{i}}{P_{i}}\right), \\
& =\frac{1}{N^{2}} \sum_{i=1}^{N} \frac{1}{P_{i}^{2}} \operatorname{Var}\left(Z_{i}\right) .
\end{aligned}
$$

The variance of the Bernoulli-type random variable $Z$ is given by $4 p q$. Hence,

$$
\operatorname{Var}(S)=\frac{4 p q}{N^{2}} \sum_{i=1}^{N} \frac{1}{P_{i}^{2}}
$$

Using $p$ and $q$ to represent the proportion of events in a +1 and -1 helicity state, respectively, $p$ and $q$ are then estimated by

$$
\begin{aligned}
& p=\frac{\frac{N^{+}}{\alpha^{+}}}{\frac{N^{+}}{\alpha^{+}}+\frac{N^{-}}{\alpha^{-}}} \equiv \frac{\bar{N}^{+}}{\bar{N}}, \\
& q=\frac{\frac{N^{-}}{\alpha^{-}}}{\frac{N^{+}}{\alpha^{+}}+\frac{N^{-}}{\alpha^{-}}} \equiv \frac{\bar{N}^{-}}{\bar{N}},
\end{aligned}
$$


where $\alpha^{ \pm}$are the factors obtained from Eq. 4.3. Hence,

$$
\begin{aligned}
\mathbb{E}(\hat{S}) & =\frac{1}{\bar{N}}\left(\frac{1}{\alpha^{+}} \sum_{i_{+}=1}^{N^{+}} \frac{1}{P_{i_{+}}}-\frac{1}{\alpha^{-}} \sum_{i_{-}=1}^{N^{-}} \frac{1}{P_{i_{-}}}\right), \\
& =\frac{1}{\bar{N}}\left(\frac{Y^{+}}{\alpha^{+}}-\frac{Y^{-}}{\alpha^{-}}\right)
\end{aligned}
$$

where $Y^{ \pm}$are as given in Eq. 4.6. This shows that $S(p, q)$ with $p, q$ given by Eqs. 4.18 and 4.19 is analogous with Eq. 4.2. The variance for this quantity is then given by

$$
\begin{aligned}
\operatorname{Var}(\hat{S}) & =\frac{4 \bar{N}^{+} \bar{N}^{-}}{\bar{N}^{4}} \sum_{i=1}^{\bar{N}} \frac{1}{P_{i}^{2}} \\
& =\frac{4 \bar{N}^{+} \bar{N}^{-}}{\bar{N}^{3}}\left\langle\frac{1}{P^{2}}\right\rangle
\end{aligned}
$$

where $\left\langle\frac{1}{P^{2}}\right\rangle$ denotes the average value of the squares of the photon polarization over all events in $\tau$. It then follows that the standard error on $S$ (and by analogy, $I^{\odot}$ ) in $\tau$ is given by

$$
\sigma_{\text {stat }}\left(I^{\odot}\right)=\frac{2 \sqrt{\bar{N}^{+} \bar{N}^{-}}}{\bar{N}^{3 / 2}}\left\langle\frac{1}{P^{2}}\right\rangle^{1 / 2} .
$$

As Eq. 4.4 is also an instance of Eq. 4.9 with $P_{i}=1$ the uncertainty on the beam-charge asymmetry is given by

$$
\sigma_{\text {stat }}\left(a_{c}\right)=\frac{2 \sqrt{N_{\pi}^{+} N_{\pi}^{-}}}{N_{\pi}^{3 / 2}} .
$$

\subsection{Systematic Uncertainties}

Systematic uncertainties arise from possible biases introduced by the methods used to analyze the data. The systematic uncertainty on the beam-helicity asymmetry from an arbitrary source of uncertainty is estimated by

$$
\delta_{\mathrm{sys}}=\sqrt{\frac{\sum_{i}\left(\frac{I_{\mathrm{nom}}^{\odot}\left(\phi_{i}\right)-I_{\mathrm{alt}}^{\odot}\left(\phi_{i}\right)}{\delta I_{\mathrm{nom}}^{\odot}\left(\phi_{i}\right)}\right)^{2}}{\sum_{i}\left(\frac{1}{\delta I_{\mathrm{nom}}^{\odot}\left(\phi_{i}\right)}\right)^{2}}}
$$


where the sum ranges over all $\phi$ bins, $I_{\text {nom }}^{\odot}$ is the nominal value, $I_{\text {alt }}^{\odot}$ is the asymmetry of a slight variation of the source, and $\delta I_{\text {nom }}^{\odot}\left(\phi_{i}\right)$ is the statistical uncertainty of the nominal $I^{\odot}$ at the $i^{\text {th }} \phi$ bin. The sources of systematic uncertainty considered were the vertex position cuts, timing cuts, multiple photon cut, confidence level cut, $\cos (\theta)$ cut, and number of bins. Each one of these cuts were varied slightly.

The nominal vertex position and timing cuts used were discussed in Section 3.1. The alternate cuts considered were to expand and contract the nominal cuts by $10 \%$. That is, the radial cut was changed from $r<2.0 \mathrm{~cm}$ to $r<2.2 \mathrm{~cm}$ and $r<1.8 \mathrm{~cm}$. The longitudinal cut was changed from $|z-90|<20 \mathrm{~cm}$ to $|z-90|<22 \mathrm{~cm}$ and $|z-90|<18 \mathrm{~cm}$. The timing cuts were changed from $|\Delta t|<1.0$ ns to $|\Delta t|<1.1$ ns and $|\Delta t|<0.9$ ns.

The multiple photon cut was discussed in Section 3.2. The alternate used was without a multiple photon cut.

The nominal confidence level cut from kinematic fitting used was $5 \%$ and is discussed in Section 3.8. The alternate cuts used were passing a $0 \%$ confidence level cut (no confidence level cut) and a 10\% confidence level cut.

The nominal $\cos \left(\theta_{x_{i} x_{j}}\right)$ used was $\left|\cos \left(\theta_{x_{i} x_{j}}\right)\right|<0.99$. The alternate cuts used were $\left|\cos \left(\theta_{x_{i} x_{j}}\right)\right|<1.0\left(\right.$ no $\cos \left(\theta_{x_{i} x_{j}}\right)$ cut $)$ and $\left|\cos \left(\theta_{x_{i} x_{j}}\right)\right|<0.98$.

As Eq. 4.26 is not well defined for different numbers of bins, a jackknife approach was taken. That is, if the nominal number of $\phi$ bins selected is $n$ and the alternate number of bins is $m$, then $|n-m|$ bins were removed from the sample with the larger number of bins. All possible combinations of removing $|n-m|$ bins were considered and averaged. In this study, the nominal number of bins considered was 16 and alternates of 15 and 17 bins were considered. The total systematic uncertainty was taken by adding the uncertainties from the different sources in quadrature as shown by

$$
\delta_{\mathrm{sys}, \mathrm{tot}}=\sqrt{\sum_{\mathrm{src}} \delta_{\mathrm{sys}, \mathrm{src}}^{2}} .
$$


Tables 4.2 to 4.5 summarize the systematic uncertainties from these sources for each configuration. Compared to $I_{\mathrm{rms}}^{\odot}$,

$$
I_{\mathrm{rms}}^{\odot}=\sqrt{\frac{\sum_{i}\left(\frac{I^{\odot}\left(\phi_{i}\right)}{\delta I^{\odot}\left(\phi_{i}\right)}\right)^{2}}{\sum_{i}\left(\frac{1}{\delta I^{\odot}\left(\phi_{i}\right)}\right)^{2}}},
$$

the relative systematic uncertainty throughout the study is $\approx 10 \%$, i.e.,

$$
\frac{\delta_{\text {sys,tot }}}{I_{\text {rms }}^{\odot}} \approx 0.1
$$

\begin{tabular}{|l|l|}
\hline Source & $\delta I^{\odot}$ \\
\hline Vertex Position & $1.57 \times 10^{-3}$ \\
Timing Cuts & $1.89 \times 10^{-3}$ \\
Multiple Photon & $2.92 \times 10^{-3}$ \\
Confidence Level & $3.06 \times 10^{-3}$ \\
$\cos \left(\theta_{\pi^{+}} \pi^{-}\right)$ & $6.09 \times 10^{-4}$ \\
Number of Bins & $7.35 \times 10^{-3}$ \\
\hline Total Systematic & $8.85 \times 10^{-3}$ \\
\hline
\end{tabular}

\begin{tabular}{|l|l|}
\hline Source & $\delta I^{\odot}$ \\
\hline Vertex Position & $2.19 \times 10^{-3}$ \\
Timing Cuts & $3.82 \times 10^{-3}$ \\
Multiple Photon & $7.10 \times 10^{-3}$ \\
Confidence Level & $7.94 \times 10^{-3}$ \\
cos $\left(\theta_{K^{+} K^{-}}\right)$ & $2.18 \times 10^{-3}$ \\
Number of Bins & $1.06 \times 10^{-2}$ \\
\hline Total Systematic & $1.58 \times 10^{-2}$ \\
\hline
\end{tabular}

Table 4.2: Systematic uncertainties for pion Table 4.3: Systematic uncertainties for kaon $I^{\odot}$ in the meson-meson configuration. $I^{\odot}$ in the meson-meson configuration. 


\begin{tabular}{|l|l|}
\hline Source & $\delta I^{\odot}$ \\
\hline Vertex Position & $2.73 \times 10^{-3}$ \\
Timing Cuts & $2.49 \times 10^{-3}$ \\
Multiple Photon & $6.75 \times 10^{-3}$ \\
Confidence Level & $6.36 \times 10^{-3}$ \\
$\cos \left(\theta_{p K^{-}}\right)$ & $3.46 \times 10^{-3}$ \\
Number of Bins & $9.07 \times 10^{-3}$ \\
\hline Total Systematic & $1.39 \times 10^{-2}$ \\
\hline
\end{tabular}

\begin{tabular}{|l|l|}
\hline Source & $\delta I^{\odot}$ \\
\hline Vertex Position & $1.53 \times 10^{-3}$ \\
Timing Cuts & $3.07 \times 10^{-3}$ \\
Multiple Photon & $8.51 \times 10^{-3}$ \\
Confidence Level & $8.13 \times 10^{-3}$ \\
$\cos \left(\theta_{p K^{+}}\right)$ & $1.84 \times 10^{-3}$ \\
Number of Bins & $1.19 \times 10^{-2}$ \\
\hline Total Systematic & $1.71 \times 10^{-2}$ \\
\hline
\end{tabular}

Table 4.4: Systematic uncertainties for kaon Table 4.5: Systematic uncertainties for kaon $I^{\odot}$ in the neutral baryon configuration. $\quad I^{\odot}$ in the positive baryon configuration.

\subsection{Conclusions}

The angular dependence of the beam-helicity asymmetry for two charged kaons in photoproduction was shown for the first time. It was also compared to the beam-helicity asymmetry for two charged pions in photoproduction, which was also a first-time measurement for energies $W>2.3 \mathrm{GeV}$. The asymmetry was also studied as functions of key kinematic variables: $W$, invariant masses, and momentum transfers. It was also studied with respect to various different plane and angle definitions. The most obvious property shown is the odd symmetry with respect to $\phi$, i.e., $I^{\odot}(-\phi)=-I^{\odot}(\phi)$. This is due to parity conservation in the reaction. As a consequence of this symmetry, $I^{\odot}$ was fitted to a Fourier sine series (Eq. 4.7) and its coefficients were studied with respect to the aforementioned kinematic variables.

Figures 4.4 , 4.8 and 4.12 show the angular dependence of the beam-helicity asymmetry in the three different configurations, summed over all other kinematics. In the meson-meson and neutral baryon configuration, the kaon asymmetry is dominated almost exclusively by 
the $\sin (\phi)$ term. This changes for the kaon asymmetry in the positive baryon configuration in which the $\sin (2 \phi)$ has a larger contribution. The differing features in the asymmetry with respect to the different plane definitions may be due to production mechanisms interfering differently. If this is so, studying the beam-helicity asymmetry with different plane definitions may be used to probe the contributions of different production mechanisms.

The asymmetry also shows certain similarities between the different configurations: (1) the magnitude of the asymmetry decreases as the invariant mass of the $K^{+} K^{-}$system increases and has a local maximum at $M\left(K^{+} K^{-}\right) \approx 1.1 \mathrm{GeV} ;(2)$ the magnitude of the asymmetry increases with the invariant mass of the $p K^{-}$; (3a) the magnitude of the asymmetry increases as the invariant mass of $p K^{+}$increases though to a lesser extent than as a function of $M\left(p K^{-}\right)$; (3b) the magnitude of the asymmetry as a function of $M\left(p K^{+}\right)$has a local maximum at $M\left(p K^{+}\right) \approx 1.5 \mathrm{GeV} ;(4)$ the magnitude of the asymmetry as a function of $t_{\gamma \rightarrow K^{+}}$reaches a maximum at $\approx 1.3 \mathrm{GeV}^{2}$ and appears to vanish at large momentum transfer.

Perhaps the most striking feature of the asymmetry is the apparent agreement of $c_{1}$ among the three different plane-angle configurations, up to a sign. This observation can be summarized by the relation

$$
c_{1}^{n b}(\tau) \approx c_{1}^{p b}(\tau) \approx-c_{1}^{m m}(\tau)
$$

where $c_{1}^{x}(\tau)$ is the leading Fourier coefficient for a given kinematic bin $\tau$ in the configuration labeled by the superscript $x$. The notation $c_{j}^{n b}$ refers to the $j^{\text {th }}$ Fourier coefficient obtained in the neutral baryon plane configuration and follows similarly for the other configurations. This property for $c_{1}$ does not occur for the other coefficients and was also observed for double-pion production in reference [52]. Figures 4.16 to 4.18 show a comparison of the Fourier coefficients for the different configurations.

In order to fully make sense of the results shown, a better theoretical understanding of the reactions is needed. While the pion reactions have been studied extensively at energies $W<2.3 \mathrm{GeV}$ and the model in reference 58 describes those reactions well, kaon reaction 
models remain sparse. Further studies to expand on these results could be done by using a different target. For instance, a deuterium target allows for the comparison for quasi-free protons and neutrons in the reaction. Also, since a helium-4 target has no spin or isospin, it would be an ideal target to measure the asymmetry coming purely from the photon's polarization as opposed to averaging out over all target's spin configurations [59]. 


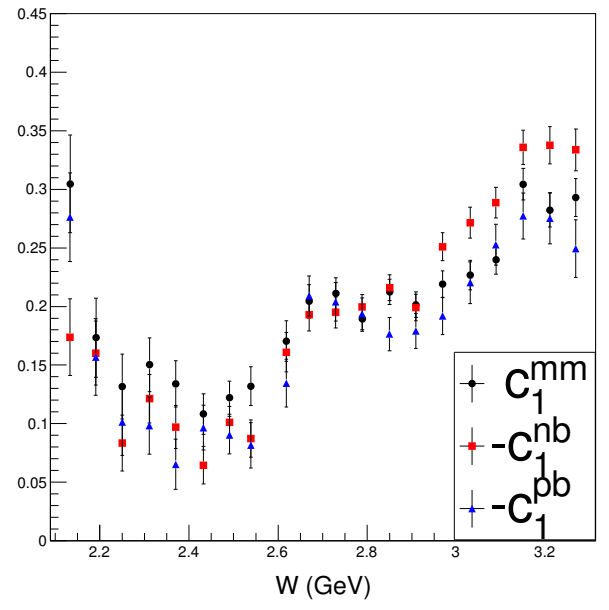

(a) $c_{1}^{x}(W)$

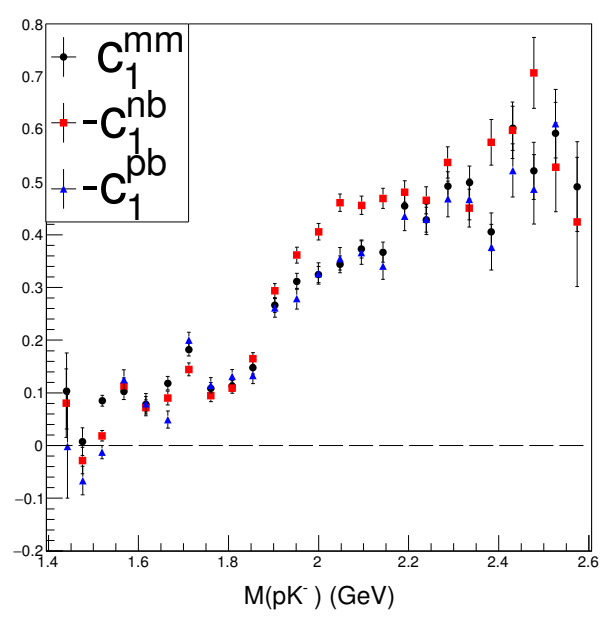

(c) $c_{1}^{x}\left(M\left(p K^{-}\right)\right)$

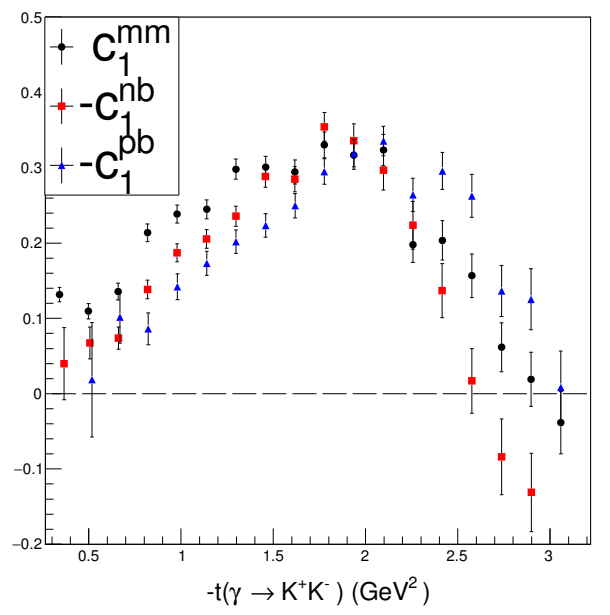

(e) $c_{1}^{x}\left(t_{\gamma \rightarrow K^{+} K^{-}}\right)$

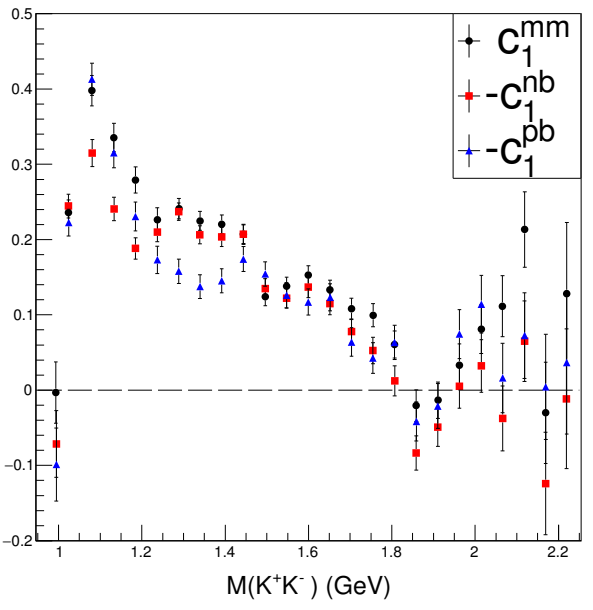

(b) $c_{1}^{x}\left(M\left(K^{+} K^{-}\right)\right)$

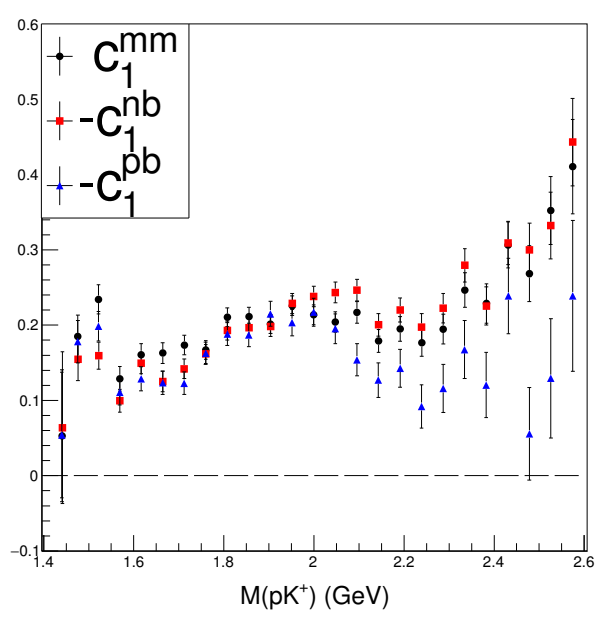

(d) $c_{1}^{x}\left(M\left(p K^{+}\right)\right)$

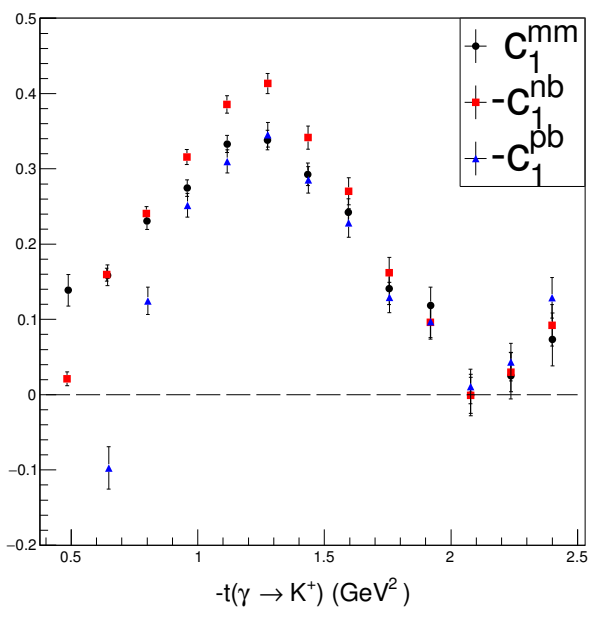

(f) $c_{1}^{x}\left(t_{\gamma \rightarrow K^{+}}\right)$

Figure 4.16: Comparison of $c_{1}$ for the three different configurations. 


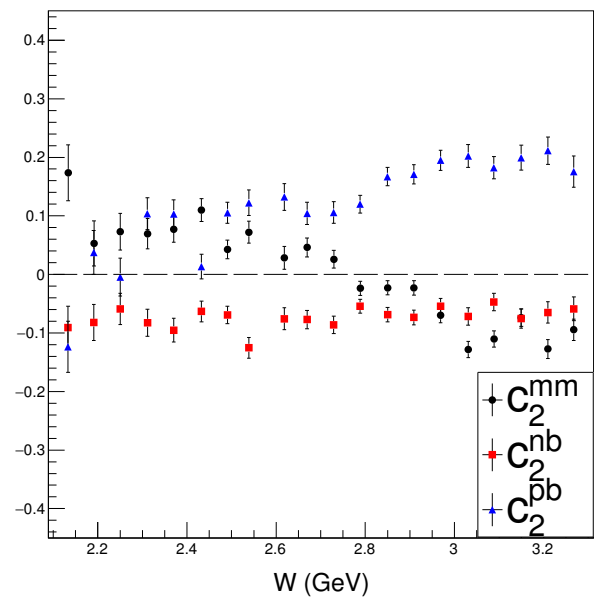

(a) $c_{2}^{x}(W)$

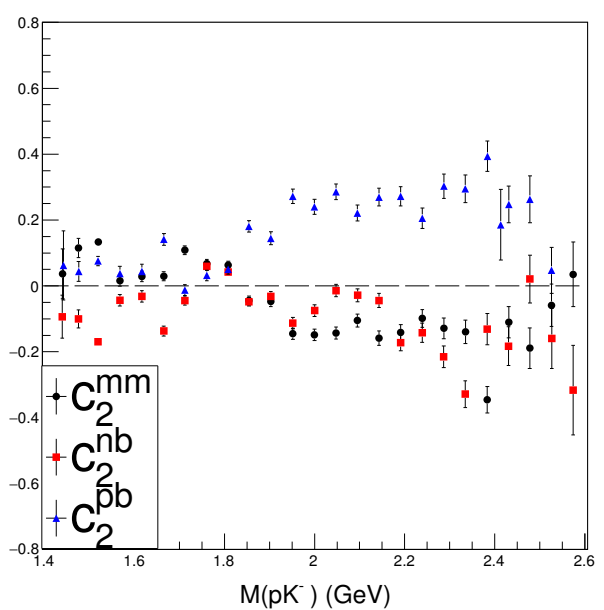

(c) $c_{2}^{x}\left(M\left(p K^{-}\right)\right)$

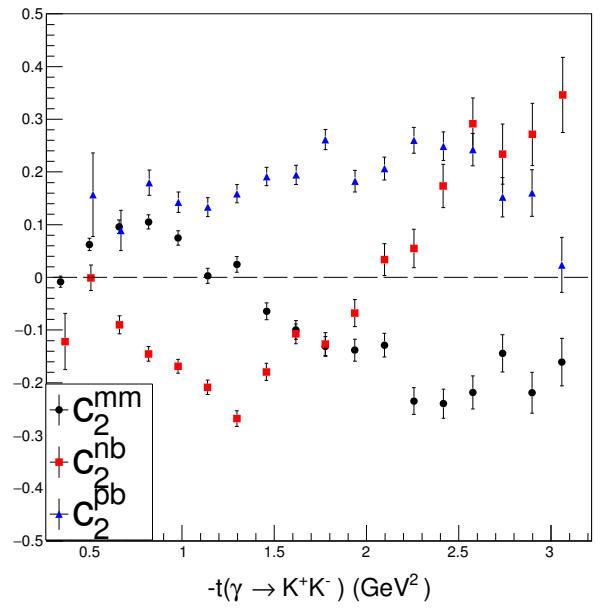

(e) $c_{2}^{x}\left(t_{\gamma \rightarrow K^{+} K^{-}}\right)$

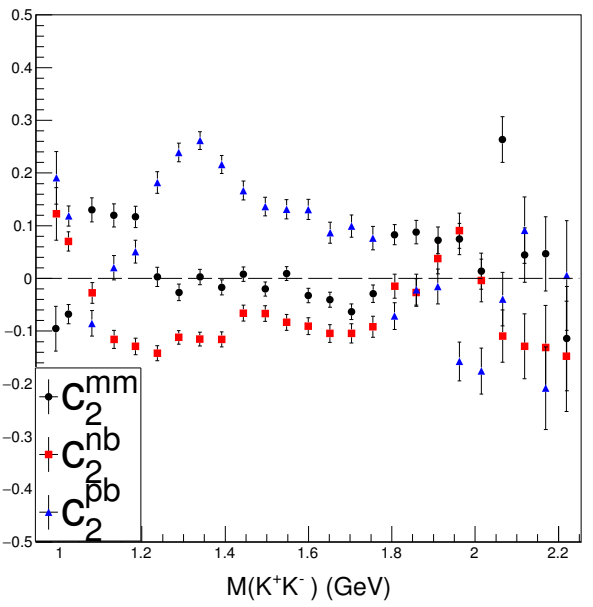

(b) $c_{2}^{x}\left(M\left(K^{+} K^{-}\right)\right)$

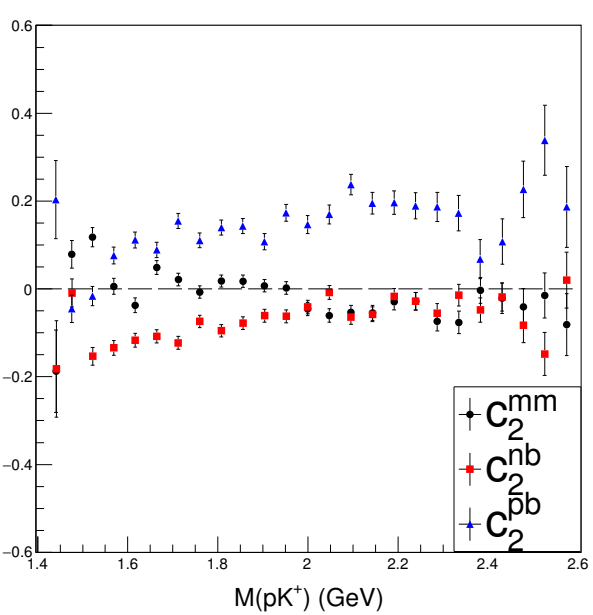

(d) $c_{2}^{x}\left(M\left(p K^{+}\right)\right)$

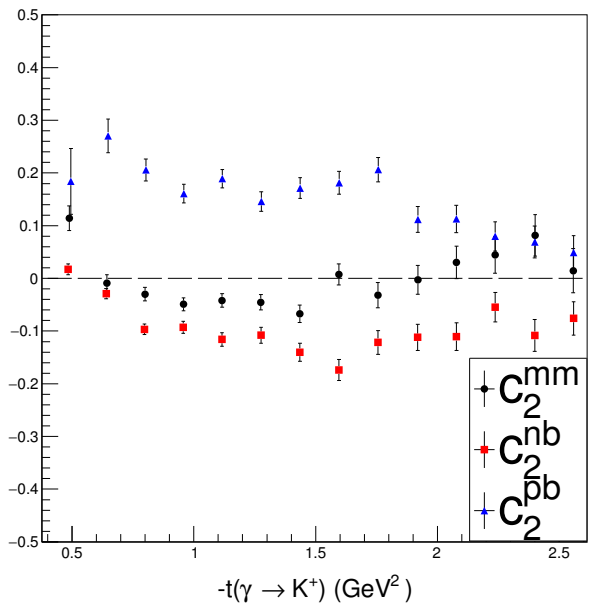

(f) $c_{2}^{x}\left(t_{\gamma \rightarrow K^{+}}\right)$

Figure 4.17: Comparison of $c_{2}$ for the three different configurations. 


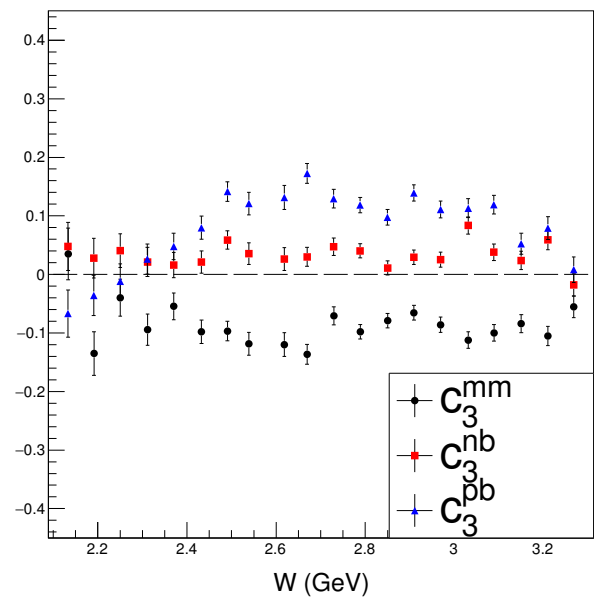

(a) $c_{3}^{x}(W)$

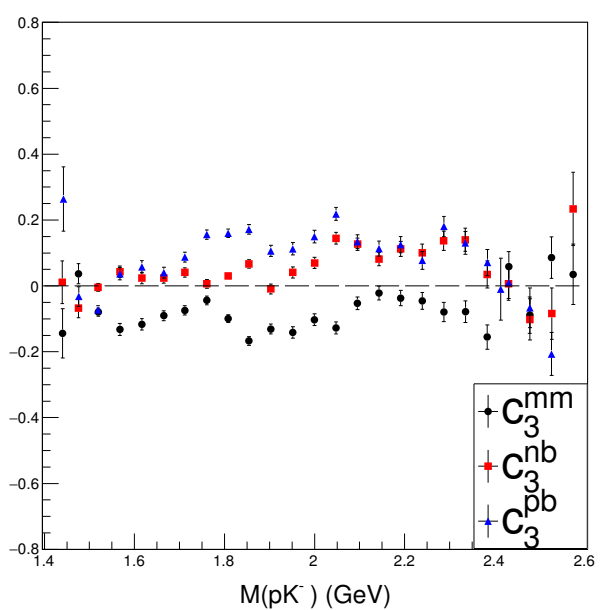

(c) $c_{3}^{x}\left(M\left(p K^{-}\right)\right)$

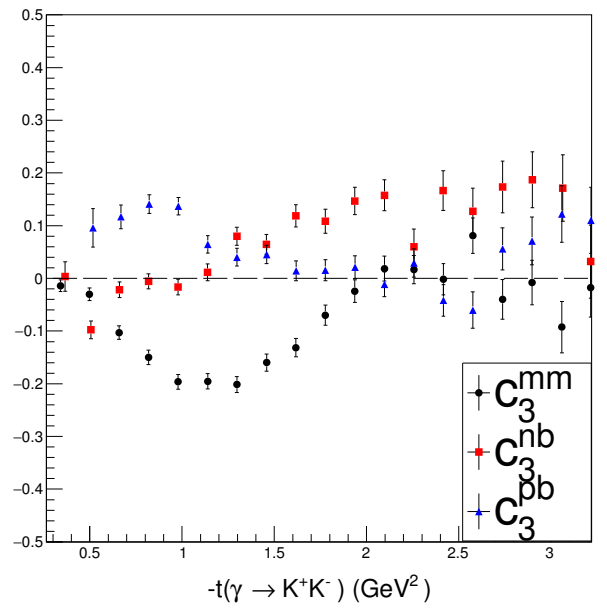

(e) $c_{3}^{x}\left(t_{\gamma \rightarrow K^{+} K^{-}}\right)$

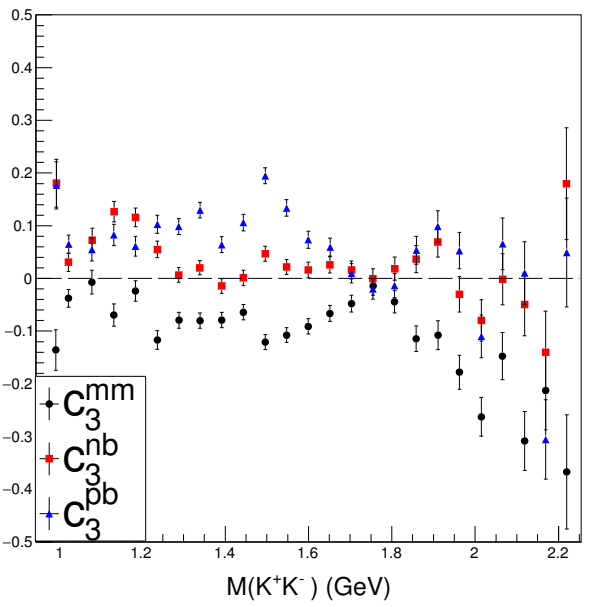

(b) $c_{3}^{x}\left(M\left(K^{+} K^{-}\right)\right)$

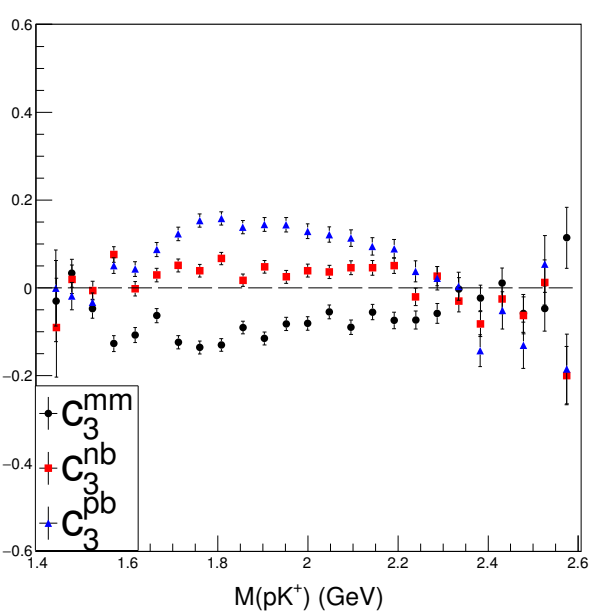

(d) $c_{3}^{x}\left(M\left(p K^{+}\right)\right)$

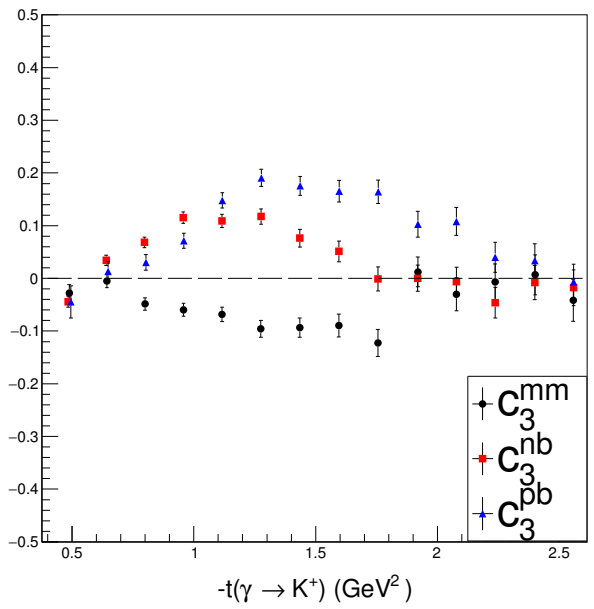

(f) $c_{3}^{x}\left(t_{\gamma \rightarrow K^{+}}\right)$

Figure 4.18: Comparison of $c_{3}$ for the three different configurations. 


\section{CHAPTER 5}

\section{Partial Wave Analysis}

Partial wave analysis (PWA) is a tool used to extract information about the intermediate states of a scattering process in terms of the measured quantities of the initial and final states. The final state particles are observed in a certain angular distribution, which is the culmination of the decays of all intermediate resonances. The PWA takes as input the four-vector momentum of the final states and outputs the amplitudes of waves with angular quantum numbers contributed by the intermediate states in a reaction.

The amplitudes are obtained by fitting to a reaction model, which is discussed in Section 5.2. The amplitudes are taken to be the parameters of a reaction model and are obtained by the maximization of the likelihood function. The derivation of the likelihood function is discussed in Section 5.3. As the likelihood function depends on the measured angular distribution of particles in the final state, the amplitudes themselves depend on the detector acceptance. In order to accurately obtain the amplitudes of the intermediate states, the detector acceptance effects must be corrected for. Acceptance corrections and simulation procedures are discussed in Section 5.4

\subsection{Preliminaries}

The PWA model (Section 5.2) relies on the canonical and helicity descriptions of states and the transformations between them. The helicity formalism for the scattering of relativistic particles with spin is based on the work in reference [60]. Consider a single massive particle with momentum $\mathbf{p}$ and total angular momentum $\mathbf{J}$. Its state in the canonical basis is given by $|j m ; p \theta \phi\rangle$, or $|j m ; \mathbf{p}\rangle$ for short, where $j$ is the angular momentum quantum number and $m$ is the angular momentum along the $z$ axis. This state can be obtained by boosting from its rest frame state along $\mathbf{p}$, i.e.

$$
|j m ; \mathbf{p}\rangle=L(\mathbf{p})|j m ; \mathbf{0}\rangle,
$$


where

$$
L(\mathbf{p})=R(\phi, \theta, 0) L_{z}(p) R^{-1}(\phi, \theta, 0) \sqrt{1}
$$

and $R(\alpha, \beta, \gamma)$ is the Euler parametrization of rotations in $\mathbb{R}^{3}$. The operation given by $L(\mathbf{p})$ first sets $\mathbf{p}$ to be along the $z$ axis, applies a boost along the $z$ axis with momentum $p$, then rotates the system back to its original coordinate system.

The helicity of the particle, $\lambda$, is defined to be the component of the particle's spin along its direction of motion, i.e.

$$
\lambda=\mathbf{J} \cdot \hat{p}
$$

In the helicity basis, the $z$ direction is taken to be along the particle's direction of motion. In this basis, since $\hat{z}=\hat{p}$,

$$
\lambda=\mathbf{J} \cdot \hat{z}=m .
$$

The particle's state can then be given as $|j \lambda ; \mathbf{p}\rangle$. Obtaining this state by boosting from its rest frame,

$$
|j \lambda ; \mathbf{p}\rangle=R(\phi, \theta, 0) L_{z}(p)|j \lambda ; \mathbf{0}\rangle \text {. }
$$

The difference between the canonical and helicity bases is that the canonical basis takes a predefined, arbitrary $z$ axis whereas the helicity basis defines its $z$ axis based on the particle's momentum. The transformation between the two bases can be obtained by inserting an identity operator, $R^{-1}(\phi, \theta, 0) R(\phi, \theta, 0)$, into Eq. 5.5, giving

$$
|j \lambda ; \mathbf{p}\rangle=R(\phi, \theta, 0) L_{z}(p) R^{-1}(\phi, \theta, 0) R(\phi, \theta, 0)|j \lambda ; \mathbf{0}\rangle .
$$

\footnotetext{
${ }^{1} L(\mathbf{p})$ is really a unitary representation of the Lorentz group acting on the Hilbert space of one-particle states. The same applies for the rotations $R(\alpha, \beta, \gamma)$.
} 
Inserting another identity operator on the space of states with angular momentum $j$ given by $\sum_{m^{\prime}}\left|j m^{\prime} ; \mathbf{0}\right\rangle\left\langle j m^{\prime} ; \mathbf{0}\right|$, it follows that

$$
\begin{aligned}
|j \lambda ; \mathbf{p}\rangle & =\sum_{m^{\prime}} R(\phi, \theta, 0) L_{z}(p) R^{-1}(\phi, \theta, 0)\left|j m^{\prime} ; \mathbf{0}\right\rangle\left\langle j m^{\prime} ; \mathbf{0}|R(\phi, \theta, 0)| j \lambda ; \mathbf{0}\right\rangle, \\
& =\sum_{m^{\prime}} D_{m^{\prime} \lambda}^{j}(\phi, \theta, 0)\left|j m^{\prime} ; \mathbf{p}\right\rangle,
\end{aligned}
$$

where

$$
D_{m \lambda}^{j}(\alpha, \beta, \gamma) \equiv\langle j m|R(\alpha, \beta, \gamma)| j \lambda\rangle
$$

are the Wigner $D$ matrices. Explicit expressions of the Wigner $D$ matrices can be found in references [61, 62. Essential properties and identities of the Wigner $D$ matrices with their derivations can also be found there. The relevant formulae for this work are shown in appendix 6 .

Consider a system of two massive particles with helicities $\lambda_{1}$ and $\lambda_{2}$ and total angular momentum $j$. Let $\mathbf{p}$ be the momentum of particle 1 in the two-particle rest frame so that particle 2 has momentum $-\mathbf{p}$. The two-particle state can be described in the helicity basis by $\left|j \lambda ; \mathbf{p} \lambda_{1} \lambda_{2}\right\rangle$ where $\lambda=\lambda_{1}-\lambda_{2}$. From Eq. 5.8, the two-particle state can be expressed in the canonical basis as

$$
\left|j \lambda ; \mathbf{p} \lambda_{1} \lambda_{2}\right\rangle=\sum_{m^{\prime}} D_{m^{\prime} \lambda}^{j}(\phi, \theta, 0)\left|j m ; \mathbf{p} \lambda_{1} \lambda_{2}\right\rangle
$$

which can be inverted using identity Eq. 6 to obtain

$$
\left|j m ; \mathbf{p} \lambda_{1} \lambda_{2}\right\rangle=\frac{2 j+1}{4 \pi} \int_{S^{2}} \mathrm{~d} \Omega D_{m \lambda}^{j *}(\phi, \theta, 0)|j \lambda ; \mathbf{p}\rangle .
$$


On the other hand, the state $|j \lambda ; \mathbf{p}\rangle$ is obtained from boosting the two particles from their respective rest frames and rotating to the desired angle, i.e.,

$$
\begin{aligned}
|j \lambda ; \mathbf{p}\rangle & =R(\phi, \theta, 0) L_{z}(p)\left|s_{1} \lambda_{1} ; \mathbf{0}\right\rangle R(\phi, \theta, 0) L_{-z}(p)\left|s_{2} \lambda_{2} ; \mathbf{0}\right\rangle, \\
& =\left|s_{1} \lambda_{1} ; \mathbf{p}\right\rangle\left|s_{2} \lambda_{2} ;-\mathbf{p}\right\rangle, \\
& =\sum_{m_{1} m_{2}} D_{m_{1} \lambda_{1}}^{s_{1}}(\phi, \theta, 0) D_{m_{2}-\lambda_{2}}^{s_{2}}(\phi, \theta, 0)\left|s_{1} m_{1} ; \mathbf{p} \lambda_{1}\right\rangle\left|s_{2} m_{2} ;-\mathbf{p} \lambda_{2}\right\rangle,
\end{aligned}
$$

where $s_{i}$ is the spin quantum number of the $i^{\text {th }}$ particle. Substituting Eq. 5.14 into Eq. 5.11,

$$
\begin{gathered}
\left|j m ; \mathbf{p} \lambda_{1} \lambda_{2}\right\rangle=\frac{2 j+1}{4 \pi} \sum_{m_{1} m_{2}} \int_{S^{2}} \mathrm{~d} \Omega D_{m \lambda}^{j *}(\phi, \theta, 0) D_{m_{1} \lambda_{1}}^{s_{1}}(\phi, \theta, 0) D_{m_{2}-\lambda_{2}}^{s_{2}}(\phi, \theta, 0) \\
\times\left|s_{1} m_{1} ; \mathbf{p} \lambda_{1}\right\rangle\left|s_{2} m_{2} ;-\mathbf{p} \lambda_{2}\right\rangle .
\end{gathered}
$$

Applying Eqs. 8 and 9, it follows that

$$
\left|j m ; \mathbf{p} \lambda_{1} \lambda_{2}\right\rangle=\sum_{l s}\left(\frac{2 l+1}{2 j+1}\right)^{1 / 2}(l 0 s \lambda \mid j \lambda)\left(s_{1} \lambda_{1} s_{2}-\lambda_{2} \mid s \lambda\right)|j m l s\rangle .
$$

\subsection{The Model}

The formalism on which this PWA is based follows from the work in references [19 21]. Consider the reaction $\gamma p \rightarrow Y^{*} K^{+} \rightarrow\left(p K^{-}\right) K^{+}$, where an excited hyperon is produced and then decays. Starting from Fermi's Golden Rule, the differential cross section is given by

$$
\frac{\mathrm{d} \sigma}{\mathrm{d} t \mathrm{~d} s \mathrm{~d} M} \propto \sum_{\text {ext. spins }} \int|\mathcal{M}|^{2} \mathrm{~d} \Omega
$$

where

$$
\begin{aligned}
t & =\left(\gamma-K^{+}\right)^{2}, \\
s & =\left(\gamma+p_{\text {target }}\right)^{2}, \\
M & =\sqrt{\left(p_{\text {scat }}+K^{-}\right)^{2}} .
\end{aligned}
$$


The quantity $\mathcal{M}$ is the Lorentz-invariant transition amplitude and $\mathrm{d} \Omega$ is a differential area element of the unit sphere in the Gottfried-Jackson fram $2^{2}$ of the $Y^{*}$. The intensity is defined as

$$
I=\sum_{\text {ext. spins }}|\mathcal{M}|^{2}
$$

where the sum is over all external spin states. Writing $\mathcal{M}$ in terms of the measurable incoming and outgoing states,

$$
\mathcal{M}=\langle\text { out }|\hat{T}| \text { in }\rangle
$$

where $\hat{T}$ is the transition operator, Eq. 5.21 becomes

$$
\begin{aligned}
I & =\sum_{\text {ext. spins }}\langle\text { out }|\hat{T}| \text { in }\rangle\langle\text { out }|\hat{T}| \text { in }\rangle^{*}, \\
& =\sum_{\text {ext. spins }}\langle\text { out }|\hat{T}| \text { in }\rangle\left\langle\text { in }\left|\hat{T}^{\dagger}\right| \text { out }\right\rangle .
\end{aligned}
$$

The transition operator can be decomposed into a part that produces a resonance and then decays it. That is,

$$
\hat{T}=\hat{T}_{\text {decay }} \hat{T}_{\text {production }}
$$

The intensity can then be expressed as

$$
I=\sum_{\text {ext. spins }}\left\langle\text { out }\left|\hat{T}_{\mathrm{d}} \hat{T}_{\mathrm{p}}\right| \text { in }\right\rangle\left\langle\operatorname{in}\left|\hat{T}_{\mathrm{p}}^{\dagger} \hat{T}_{\mathrm{d}}^{\dagger}\right| \text { out }\right\rangle
$$

Figure 5.1 shows a schematic of the transition operator and its composition in relation to the reaction $\gamma p \rightarrow p K^{+} K^{-}$producing an intermediate hyperon resonance.

\footnotetext{
${ }^{2}$ The Gottfried-Jackson frame is defined to be the frame in which the produced resonance, $Y^{*}$, is at rest with the $z$ axis defined to be parallel to the beam. The $y$ axis is chosen to be parallel to $\gamma \times \mathbf{p}_{Y^{*}}$ in the center-of-mass frame. Boosting to the rest frame of the $Y^{*}$ does not change this vector as it is perpendicular to the boost. The $x$ axis is chosen to form a right-handed coordinate system.
} 


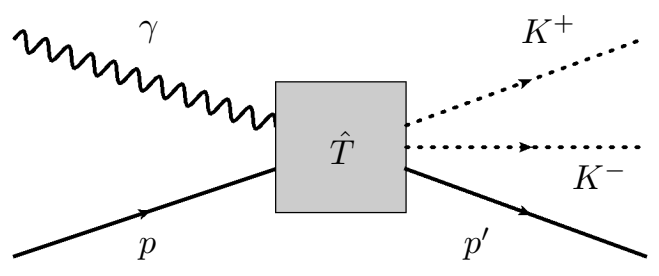

(a) Transition operator between in and out states

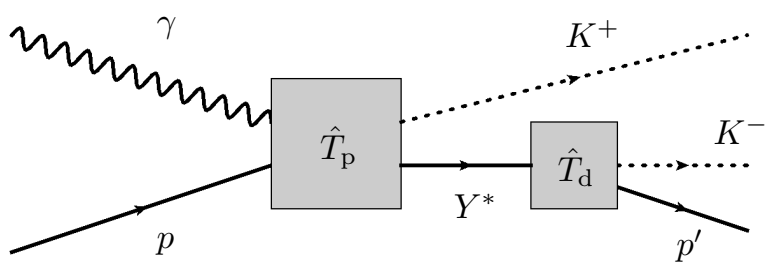

(b) Decomposition of transition operator into production and decay operators

Figure 5.1: Schematic representation of the transition operator.

Given a set of orthogonal states $|X\rangle$ with $\sum_{X}|X\rangle\langle X|=I$, Eq. 5.26 can be expanded in terms of these states. That is,

$$
\left.I=\sum_{\text {ext. spins } X, X^{\prime}} \sum_{\text {out } \mid}\left|\hat{T}_{\mathrm{d}}\right| X\right\rangle\left\langle X\left|\hat{T}_{\mathrm{p}}\right| \text { in }\right\rangle\left\langle\text { in }\left|\hat{T}_{\mathrm{p}}^{\dagger}\right| X^{\prime}\right\rangle\left\langle X^{\prime}\left|\hat{T}_{\mathrm{d}}^{\dagger}\right| \text { out }\right\rangle
$$

The orthogonal states $|X\rangle$ are called partial waves. Each of these states can, in turn, be described by a set of quantum numbers. The term $\left\langle\right.$ out $\left.\left|\hat{T}_{\mathrm{d}}\right| X\right\rangle$ is called the decay amplitude for the wave $X$ while $\left\langle X\left|\hat{T}_{\mathrm{p}}\right|\right.$ in $\rangle$ is the production amplitude for the wave $X$.

\subsubsection{Decay Amplitudes}

As mentioned previously, $\mathrm{d} \Omega$ is a differential area element of the unit sphere in the Gottfried-Jackson frame. The Gottfried-Jackson frame is the frame in which the produced resonance is at rest and the $z$ axis is chosen to be parallel to the beam. Since the produced resonance is at rest in this frame, the decay products scatter antiparallel to one another. Hence, the angles for one of the decay products is sufficient to describe the angular distribution of the decay. Let $\theta$ be the angle with respect to the $z$ axis and $\phi$ be the azimuthal angle of one of the decay products. Writing the "out" state in the helicity basis and labeling the partial waves by their quantum numbers, the decay amplitude becomes

$$
\left\langle\text { out }\left|\hat{T}_{\mathrm{d}}\right| X\right\rangle=\sum_{j^{\prime} \lambda_{1}^{\prime} \lambda_{2}^{\prime}} c_{\lambda_{1}^{\prime} \lambda_{2}^{\prime}}^{j^{\prime}}\left\langle j^{\prime} \lambda^{\prime} ; \mathbf{p} \lambda_{1}^{\prime} \lambda_{2}^{\prime}\left|\hat{T}_{\mathrm{d}}\right| j m\right\rangle,
$$


where $\lambda^{\prime}=\lambda_{1}^{\prime}-\lambda_{2}^{\prime}$. Applying Eq. 5.10,

$$
\left\langle\text { out }\left|\hat{T}_{\mathrm{d}}\right| X\right\rangle=\sum_{j^{\prime} m^{\prime} \lambda_{1}^{\prime} \lambda_{2}^{\prime}} c_{\lambda_{1}^{\prime} \lambda_{2}^{\prime}}^{j^{\prime}} D_{m^{\prime} \lambda^{\prime}}^{j^{\prime} *}(\phi, \theta, 0)\left\langle j^{\prime} m^{\prime} ; \mathbf{p} \lambda_{1}^{\prime} \lambda_{2}^{\prime}\left|\hat{T}_{\mathrm{d}}\right| j m\right\rangle
$$

Since $\hat{T}_{\mathrm{d}}$ preserves $j$ and $m$, the sums over $j^{\prime}$ and $m^{\prime}$ can be eliminated, as the only contribution will be when $j^{\prime}=j$ and $m^{\prime}=m$. Inserting the identity operator $\sum_{l^{\prime} s^{\prime}}\left|j m l^{\prime} s^{\prime}\right\rangle\left\langle j m l^{\prime} s^{\prime}\right|$ to consider the outgoing angular momentum contributions,

$$
\left\langle\text { out }\left|\hat{T}_{\mathrm{d}}\right| X\right\rangle=\sum_{\lambda_{1}^{\prime} \lambda_{2}^{\prime} l^{\prime} s^{\prime}} c_{\lambda_{1}^{\prime} \lambda_{2}^{\prime}}^{j} D_{m \lambda^{\prime}}^{j *}(\phi, \theta, 0)\left\langle j m ; \mathbf{p} \lambda_{1}^{\prime} \lambda_{2}^{\prime} \mid j m l^{\prime} s^{\prime}\right\rangle\left\langle j m l^{\prime} s^{\prime}\left|\hat{T}_{\mathrm{d}}\right| j m\right\rangle
$$

The term $\left\langle j m l s\left|\hat{T}_{\mathrm{d}}\right| j m\right\rangle$ is known as the transition amplitude and will be labeled as $a_{l s}^{j m}$. Applying Eq. 5.16 .

$$
\left\langle\text { out }\left|\hat{T}_{\mathrm{d}}\right| X\right\rangle=\sum_{\lambda_{1}^{\prime} \lambda_{2}^{\prime} l^{\prime} s^{\prime}} c_{\lambda_{1}^{\prime} \lambda_{2}^{\prime}}^{j}\left(\frac{2 l^{\prime}+1}{2 j+1}\right)^{1 / 2} D_{m \lambda^{\prime}}^{j *}(\phi, \theta, 0)(l 0 s \lambda \mid j \lambda)\left(s_{1} \lambda_{1} s_{2}-\lambda_{2} \mid s \lambda\right) a_{l s}^{j m}
$$

The $c_{\lambda_{1}^{\prime} \lambda_{2}^{\prime}}^{j}$ are fixed by normalization. From Eq. 6 , it follows that

$$
c_{\lambda_{1}^{\prime} \lambda_{2}^{\prime}}^{j}=\left(\frac{2 j+1}{4 \pi}\right)^{1 / 2} .
$$

Finally, the decay amplitude can be written as

$$
\left\langle\text { out }\left|\hat{T}_{\mathrm{d}}\right| X\right\rangle=\sum_{\lambda_{1}^{\prime} \lambda_{2}^{\prime} l^{\prime} s^{\prime}}\left(\frac{2 l^{\prime}+1}{4 \pi}\right)^{1 / 2} D_{m \lambda^{\prime}}^{j *}(\phi, \theta, 0)(l 0 s \lambda \mid j \lambda)\left(s_{1} \lambda_{1} s_{2}-\lambda_{2} \mid s \lambda\right) a_{l s}^{j m} .
$$

\subsubsection{Reflectivity Basis}

As the strong interactions conserve parity, this imposes a constraint on the $p K^{-}$decay. However, the helicity states defined in Section 5.1 are not eigenstates of the parity operator and do not satisfy this constraint. The basis that demonstrates this symmetry is known as the reflectivity basis. 
The parity operator, $\hat{\Pi}$, is the operator that takes $\mathbf{r}$ to $-\mathbf{r}$. Consequently, it does not change the total angular momentum but $\mathbf{p} \rightarrow-\mathbf{p}$. It then follows that $\lambda \rightarrow-\lambda$ under a parity transformation. Consider a state with spin $j$ and momentum $p$ in the helicity basis. Then,

$$
\begin{aligned}
\hat{\Pi}|j \lambda ; p\rangle & =P|j-\lambda ;-p\rangle, \\
& =P R(0, \pi, 0)|j \lambda ; p\rangle .
\end{aligned}
$$

Since $\hat{\Pi}^{2}=I$, it follows that $P= \pm 1$. Introducing the operator

$$
\hat{\Pi}_{y}=\hat{\Pi} R(0, \pi, 0)
$$

it follows that $|j \lambda ; p\rangle$ is an eigenstate of $\hat{\Pi}_{y}$. This operator is called the reflectivity operator. Physically, it is a reflection through the production plane. Applying the reflectivity operator on the canonical states,

$$
\hat{\Pi}_{y}|j m\rangle=\hat{\Pi} R(0, \pi, 0)|j m\rangle
$$

and inserting the identity operator $\sum_{m^{\prime}}\left|j m^{\prime}\right\rangle\left\langle j m^{\prime}\right|$, it follows that

$$
\begin{aligned}
\hat{\Pi}_{y}|j m\rangle & =\sum_{m^{\prime}} \hat{\Pi}\left|j m^{\prime}\right\rangle\left\langle j m^{\prime}|R(0, \pi, 0)| j m\right\rangle \\
& =\sum_{m^{\prime}} P_{m^{\prime}}\left|j m^{\prime}\right\rangle d_{m-m^{\prime}}^{j}(-\pi)
\end{aligned}
$$

Using the identity in Eq. 11 .

$$
\hat{\Pi}_{y}|j m\rangle=P(-1)^{j-m}|j-m\rangle .
$$


This suggests that states of the form $c_{1}|j m\rangle+c_{2}|j-m\rangle$ are eigenstates of the reflectivity operator. Solving the eigenvalue problem,

$$
\begin{aligned}
\hat{\Pi}_{y}\left[c_{1}|j m\rangle+c_{2}|j-m\rangle\right] & =\epsilon\left[c_{1}|j m\rangle+c_{2}|j-m\rangle\right], \\
\hat{\Pi}_{y}\left[c_{1}|j m\rangle+c_{2}|j-m\rangle\right] & =P\left[c_{1}(-1)^{j-m}|j-m\rangle+c_{2}(-1)^{j+m}|j m\rangle\right] .
\end{aligned}
$$

Hence,

$$
\left\{\begin{array}{l}
\epsilon c_{1}=c_{2}(-1)^{j+m} \\
\epsilon c_{2}=c_{1}(-1)^{j-m}
\end{array}\right.
$$

If $\epsilon=0$, then $c_{1}=c_{2}=0$, which would yield the trivial solution. Also, if either $c_{1}$ or $c_{2}=0$, then that too will yield the trivial solution. It then follows that

$$
\epsilon=(-1)^{j}
$$

The eigenvalue, $\epsilon$, is called the reflectivity. The constant is obtained by normalization and is given by

$$
\left\{\begin{array}{l}
c_{1}=\frac{1}{\sqrt{2}} \text { if } m>0 \\
c_{1}=\frac{1}{2} \text { if } m=0 \\
c_{1}=0 \text { if } m<0
\end{array}\right.
$$

The eigenstates of the reflectivity operator in terms of the canonical basis states are expressed as

$$
|\epsilon j m\rangle \equiv\left[|j m\rangle+\frac{P}{\epsilon}(-1)^{j-m}|j-m\rangle\right] \Theta(m),
$$

where $\Theta(m)$ is the normalization constant from Eq. 5.45. The inverse of Eq. 5.46 is given by

$$
|j m\rangle=\sum_{\epsilon}|\epsilon j m\rangle \Theta(m)+\epsilon^{*} P(-1)^{j+m}|\epsilon j m\rangle \Theta(-m)
$$

Expressing the production and decay amplitude in the reflectivity basis, the intensity function is expressed as

$$
I(\theta, \phi ; \mathbf{V})=\sum_{\substack{\epsilon \epsilon^{\prime} \\ b b^{\prime}}} A_{\epsilon b} V_{\epsilon b} \rho_{\epsilon \epsilon^{\prime}} V_{\epsilon^{\prime} b^{\prime}}^{*} A_{b^{\prime}}^{*}
$$


where $b$ is the set of quantum numbers $\left\{\lambda_{p}, \lambda_{K^{-}}, j, m, l, s\right\}, A_{\epsilon b}$ is the decay amplitude from Eq. 5.33 without the transition amplitude term, in the reflectivity basis, and $V_{\epsilon b} \rho_{\epsilon \epsilon^{\prime}} V_{\epsilon^{\prime} b^{\prime}}^{*}$ is the production amplitude with the transition amplitude absorbed into the $V_{\epsilon b}$. The information needed for $A$ and $\rho$ is obtained from the data. The $V$ terms are unknown and are estimated using the maximum likelihood estimation method described in Section 5.3 .

\subsection{Extended Likelihood Function}

The maximum likelihood method is a method of parameter estimation for a given model with a set of parameters and set of observations. Let a be the parameters for a class of probability distributions and let $\mathbf{x}$ be a set of independent measurements. From Bayes' theorem,

$$
p(\mathbf{a} \mid \mathbf{x}) \propto p(\mathbf{x} \mid \mathbf{a}) p(\mathbf{a})
$$

The likelihood function is defined to be

$$
\mathcal{L}(\mathbf{a} ; \mathbf{x}) \equiv p(\mathbf{x} \mid \mathbf{a})
$$

Relaxing the condition that $\mathcal{L}$ be normalized yields the extended likelihood function,

$$
\mathcal{L}(\mathbf{a}, \mathcal{N} ; \mathbf{x}) \equiv \tilde{p}(N ; \mathcal{N}) p(\mathbf{x} \mid \mathbf{a})
$$

where $\mathcal{N}$ is the expected number of events to be observed in phase space and $\tilde{p}(N ; \mathcal{N})$ is the probability distribution for the number of events observed. $\tilde{p}(N ; \mathcal{N})$ is assumed to follow a Poisson distribution. Using the fact that the observed events were independent, the likelihood function becomes

$$
\mathcal{L}(\mathbf{a}, \mathcal{N} ; \mathbf{x}, N)=\frac{\mathcal{N}^{N}}{N !} e^{-\mathcal{N}} \prod_{i=1}^{N} p\left(x_{i} \mid \mathbf{a}\right)
$$

Using the intensity function given in Eq. 5.48 as the assumed probability distribution for the hyperon decay angular distribution and $\mathbf{V}$ as the parameters for the model, it follows 
that

$$
\begin{aligned}
\mathcal{L}(\mathbf{V}, \mathcal{N} ; N) & =\frac{\mathcal{N}^{N}}{N !} e^{-\mathcal{N}} \prod_{i=1}^{N} \frac{I\left(\theta_{i}, \phi_{i} ; \mathbf{V}\right)}{\mathcal{N}} \\
& =\frac{e^{-\mathcal{N}}}{N !} \prod_{i=1}^{N} I\left(\theta_{i}, \phi_{i} ; \mathbf{V}\right)
\end{aligned}
$$

where $N$ is the number of measurements. Reference [63] asserts that the parameter estimation by maximizing the likelihood function is at least asymptotically unbiased and efficient. That is, if $\mathbf{a}_{N}$ is the parameter estimate for a with $N$ measurements obtained by maximizing $\mathcal{L}$, then

$$
\begin{array}{r}
\lim _{N \rightarrow \infty} \mathbb{E}\left(\mathbf{a}_{N}\right)=\mathbf{a} \\
\text { and } \\
\lim _{N \rightarrow \infty} \frac{\mathrm{MVB}}{\operatorname{Var}\left(\mathbf{a}_{N}\right)}=1,
\end{array}
$$

where MVB is the minimum variance bound as given in reference [64]. The minimum variance bound is the smallest variance an estimator can have for a deterministic parameter. An estimator being efficient means that it attains this minimum variance and is a desired property.

The measured values can be taken to be either the proton or $K^{-}$angle in the GottfriedJackson frame, which determine the parameters. To make the computation more efficient, the logarithm of the likelihood function is maximized instead. As the logarithm function is monotonically increasing, maximization of the likelihood and log-likelihood yield the same result. Equivalently, maximizing the log-likelihood is the same as minimizing the negative. The minimization of the negative log-likelihood function is done using MINUIT [65, 66].

\subsection{Acceptance Corrections and Normalization}

As measurements of physical observables depend on the apparatus used, the effects the apparatus has on these observables should be corrected for. One such effect is the 
detector acceptance. Every detector component has a certain efficiency in detecting particles passing through it. As every component is made up of many subcomponents, this efficiency is not expected to be uniform. For example, the TOF paddles detection efficiency and timing resolution was studied in Section 3.4. Consequently, several events corresponding to particles passing through an inefficient detector had a high probability of not being detected and then ignored in the analysis. Furthermore, even the most reliable detector components are not perfect.

The acceptance is the probability that the CLAS detector will detect and reconstruct an event. The acceptance is a function principally of momentum, charge, and scattering angle. In order to estimate the acceptance, $\gamma p \rightarrow p K^{+} K^{-}$events were generated pseudorandomly using a program called genr8. genr8 takes as input the $t$ slope of the reaction (obtained from pwa:eq:tslope), the mass of either an intermediate baryon or meson resonance, and its width under the assumption that the $p K^{-}$came from a $Y^{*}$ that was produced diffractively. For this study, events were generated to approximate the beam energy distribution and the reaction's $t$ slope within that energy range. To obtain the input parameters for genr8, the data were binned into 77 equal-sized $E_{\gamma}$ bins. The $t$ slope for each bin is obtained from the assumption that

$$
\frac{\mathrm{d} \sigma}{\mathrm{d} t} \propto e^{-b t}
$$

where $\frac{\mathrm{d} \sigma}{\mathrm{d} t}$ is the differential cross section with respect to $t$ and $b$ is the $t$ slope. The generation of events was an iterative process and the parameters were obtained as follows: the $t$ slope for the generated events for the $j+1$ iteration in bin $\tau$ was computed as

$$
\begin{aligned}
N_{j+1}^{\text {gen }}(\tau) & =\left(\frac{N_{\text {data }}^{\text {dat }}}{N_{j}^{\text {rec }}(\tau)} N_{j}^{\text {gen }}(\tau)\right)\left(\frac{N_{j}^{\text {rec, total }}}{N^{\text {data, total }}}\right), \text { and } \\
b_{j+1}^{\text {gen }}(\tau) & =\frac{b^{\text {data }}(\tau)}{b_{j}^{\text {rec }}(\tau)} b_{j}^{\text {gen }}(\tau) .
\end{aligned}
$$

For each bin, the events were generated with an intermediate hyperon decaying into a proton and $K^{-}$having a large width so that the $M\left(p K^{-}\right)$distribution in a given $E_{\gamma}$ bin 
is approximately uniform. After generation, the events were then converted by gamp2part to a suitable format to be read by the next program called gsim. gsim is a GEANT ${ }^{3}$-based simulation program used to simulate the detector response of the generated events. For each generated event, it sets ADC and TDC values for each detector hit. gpp (gsim postprocessor) smears the simulated signals and simulates the response of the DC wires. The simulated events are then passed into a1c to be reconstructed. Finally, the reconstructed events are analyzed identically to how the real data were analyzed. The acceptance for a given bin, $\tau$, is then defined to be

$$
\eta(\tau)=\frac{N^{\mathrm{rec}}(\tau)}{N^{\operatorname{gen}}(\tau)}
$$

where $N^{\text {rec }}(\tau)$ is the number of reconstructed events and $N^{\text {gen }}(\tau)$ is the number of generated events in $\tau$. The value, $\mathcal{N}$, in Eq. 5.54 is readily obtained from

$$
\begin{aligned}
\mathcal{N}(\tau) & =\int_{\tau} \mathrm{d} \tau^{\prime} I\left(\tau^{\prime}\right) \eta\left(\tau^{\prime}\right), \\
& \approx \frac{1}{N^{\text {gen }}} \sum_{i=1}^{N^{\text {gen }}} I\left(\tau_{i}\right) \eta\left(\tau_{i}\right),
\end{aligned}
$$

where

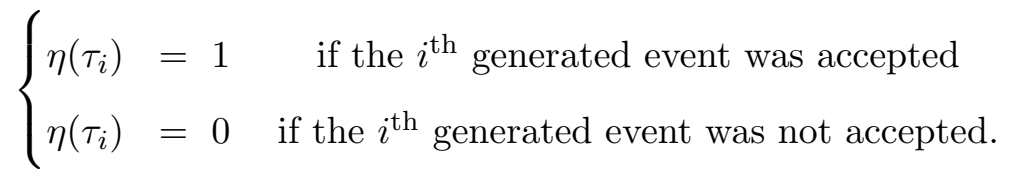

\subsection{Performing the Fit}

The software framework used to perform the partial wave analysis was the pyPWA framework developed by the group in references [67, 68]. The software takes as input the experimental, generated Monte-Carlo, and reconstructed Monte-Carlo events in gamp format 69 . It also takes as input a collection of waves to be included in the minimization of the negative log-likelihood function as keyfiles [69]. Figures 5.2 and 5.3 show an example of a gamp-formatted event and a keyfile, respectively. The keyfile shown is for a $J=\frac{3}{2} P$

\footnotetext{
${ }^{3}$ GEometry ANd Tracking
} 
wave with reflectivity $\epsilon=+1$. While the keyfile also takes the parity quantum number into consideration, in the decay of an excited hyperon into a proton and $K^{-}$, the parity of the hyperon is ambiguous as the decay amplitudes are invariant under parity transformations. This ambiguity is known as the Minami ambiguity [70, 71]. As baryons are not their own antiparticle, they do not have a $\mathrm{C}$ parity quantum number.

4

10003.237183 .23718

$141-0.1231150 .03007840 .4935061 .06388$

$1110.181896-0.2148862 .425182 .49141$

$12-1-0.05878070 .1848070 .3178840 .619553$

Figure 5.2: Example of a gamp-formatted event. The first number at the top is the number of particles in the event. The subsequent rows contain the particle ID followed by the four-vector information of that particle.

$$
\begin{aligned}
& \text { channel }=\mathrm{t} \text {; } \\
& \text { mode=binary; } \\
& .707 *( \\
& \mathrm{J}=3 \mathrm{P}=-1 \mathrm{M}=3\{ \\
& \mathrm{p}+[1] \\
& \mathrm{K}-[1] \\
& l=2 \\
& \text { \} } \\
& + \\
& \mathrm{J}=3 \mathrm{P}=-1 \mathrm{M}=-3\{ \\
& \mathrm{p}+[1] \\
& \mathrm{K}-[1] \\
& l=2
\end{aligned}
$$

Figure 5.3: Example of a keyfile. The wave shown is for a $J=M=3 / 2$ $P$-wave with reflectivity $\epsilon=+1$. Note the angular momentum quantum numbers are doubled in the keyfile.

\subsubsection{Additional Cuts for PWA}

While many hyperons are produced through the process $\gamma p \rightarrow Y^{*} K^{+} \rightarrow p K^{-} K^{+}$, there is a background process leading to the same final state: $\gamma p \rightarrow p X \rightarrow p K^{+} K^{-}$, the production of a meson resonance, $X$. The $\phi(1020)$ can be clearly seen in the $K^{+} K^{-}$ invariant mass shown in Fig. 5.4. As this resonance is fairly narrow, the vast majority of these events can be removed quite easily. Removing all events with $K^{+} K^{-}$invariant mass 
less than $1.1 \mathrm{GeV}$ removes the $\phi(1020)$ peak. This cut is shown in Fig. 5.4. However, wider and more massive mesonic resonances can still contribute and cannot be removed simply by making a mass cut. Instead, these mesonic contributions were suppressed by selecting events with small momentum transfers from the photon to the $K^{+}$. Given a kinematic bin, $\tau$, a small momentum transfer is defined to be a standard deviation from $t^{\prime}$ where

$$
t^{\prime}=t-t_{\min }
$$

and

$$
\left|t_{\min }\right|=m_{Y^{*}}^{2}-2 E_{\gamma} E_{Y^{*}}+2 E_{\gamma}\left|p_{Y^{*}}\right|
$$

A standard deviation is determined by the reciprocal of the $t$ slope: $\sigma=\frac{1}{b}$, where $\sigma$ here denotes the standard deviation. The $t$ slope was obtained for each $p K^{-}$mass bin by fitting the $t^{\prime}$ distribution to the function $A e^{-b t^{\prime}}$, where $A$ and $b$ are constants to be determined by the fit. As the $t$ slope was obtained independently for each $M\left(p K^{-}\right)$, the momentum transfer cut consequently depended on $M\left(p K^{-}\right)$. Figure 5.5 shows an example of this fit for $\left|M\left(p K^{-}\right)-1520\right|<5 \mathrm{MeV}$, which is the bin with the most events.

In addition to this small $t$ cut, a Van Hove 72 sector cut was utilized as well. Figure 5.6 shows the Van Hove plot for $\gamma p \rightarrow p K^{+} K^{-}$. The Van Hove plot considers the final state particles' longitudinal momentum, i.e., the component of the momentum along the direction of the beam, in the overall rest frame. As the net momentum is zero, the net longitudinal momentum of the final state particles is also zero. Accordingly, two of the three final state particles' longitudinal momenta must have the same sign. The Van Hove plot utilizes this property by showing which two particles have the same longitudinal momentum direction and whether it is along the direction of the beam or against it. This splits the plot into six sectors. The sectors of the Van Hove plot are labeled with the following convention: the sector directly above the traditional positive $x$ axis is Sector I. The subsequent sectors in the counterclockwise direction are labeled Sectors II, III, etc. Figure 5.6 shows the sectors of the Van Hove plot with labels for which particles have longitudinal components along or 


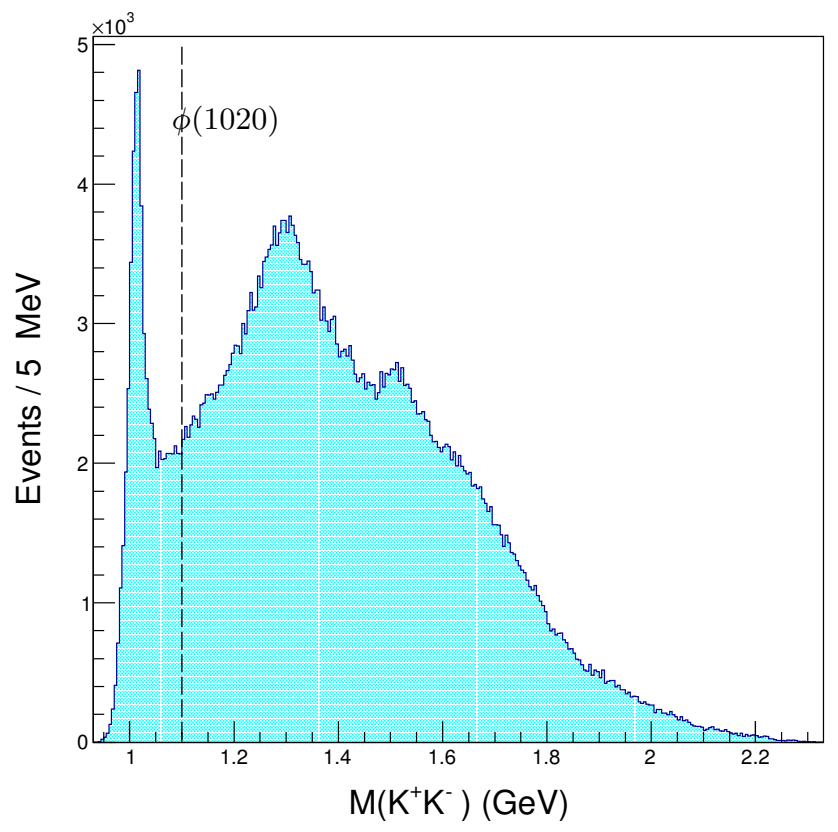

Figure 5.4: Invariant mass of $K^{+} K^{-}$

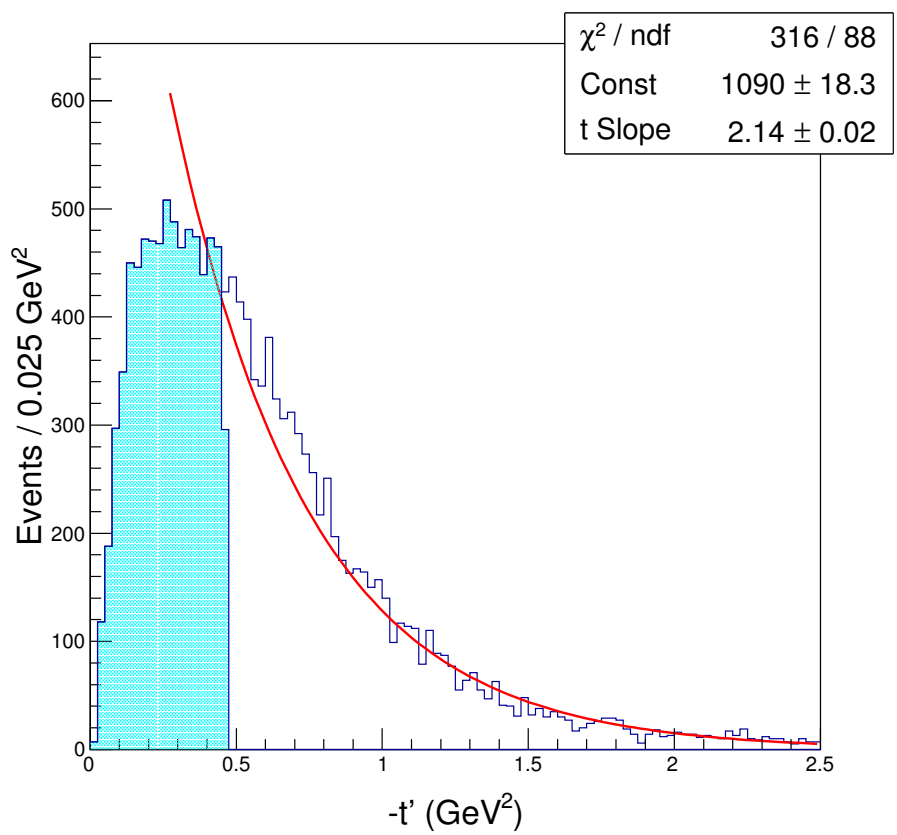

Figure 5.5: $t^{\prime}$ distribution with its fit. The shaded region shows which events survive the cut. 


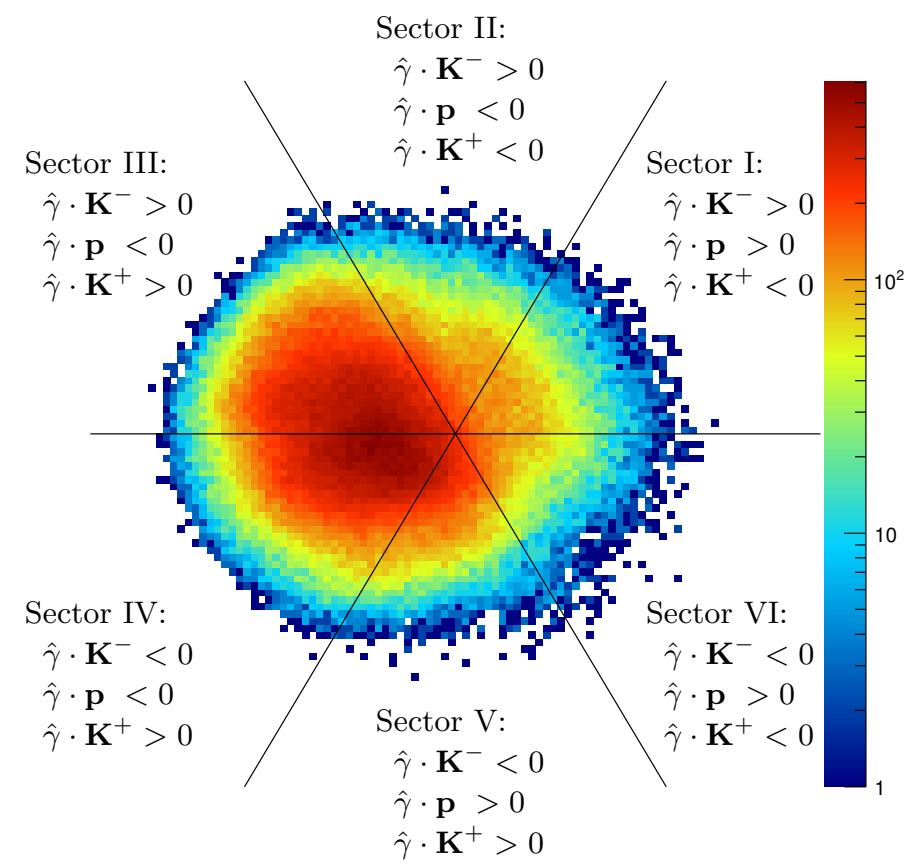

Figure 5.6: Van Hove plot for $\gamma p \rightarrow p K^{+} K^{-}$.

against the beam. The Van Hove sector cut used was

$$
(\hat{\gamma} \cdot \mathbf{p})\left(\hat{\gamma} \cdot \mathbf{K}^{-}\right)>0
$$

corresponding to events where the scattered proton and $K^{-}$have the same longitudinal direction. This is given by Sectors I and IV in Fig. 5.6. Figure 5.7 shows the effects of the Van Hove sector cut on the invariant mass spectrum of the $p K^{-}$and $K^{+} K^{-}$systems. With this cut, the signal-to-background for the $\Lambda(1520)$ is significantly enhanced, but also reduces the signal in the higher mass regions. At the same time, the invariant mass spectrum of the $K^{+} K^{-}$is slightly smeared but meson resonances still appear to be contributing. As these cuts do not completely remove the background meson production, a noninteracting background term was added to the intensity function in an attempt to absorb any remaining 


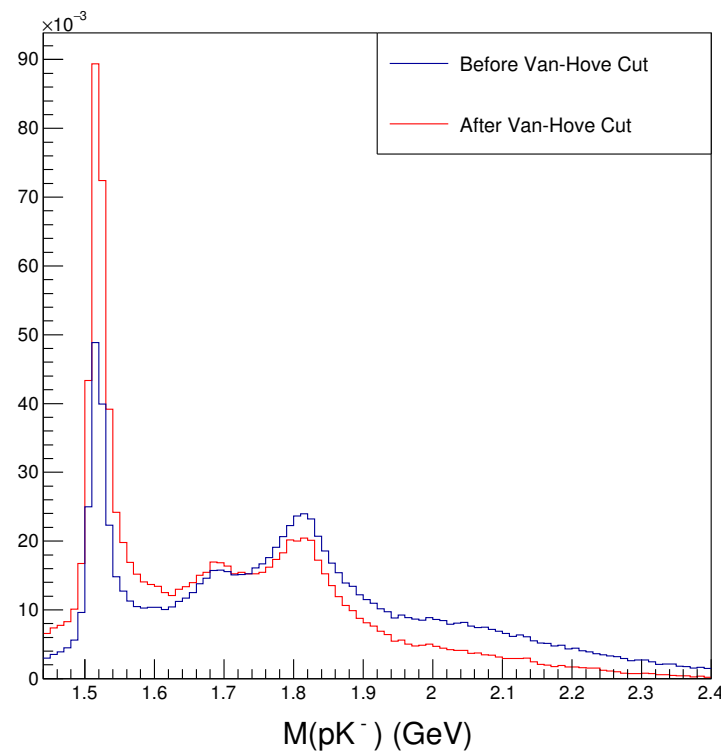

(a) $M\left(p K^{-}\right)$comparison before and after Van Hove sector cut.

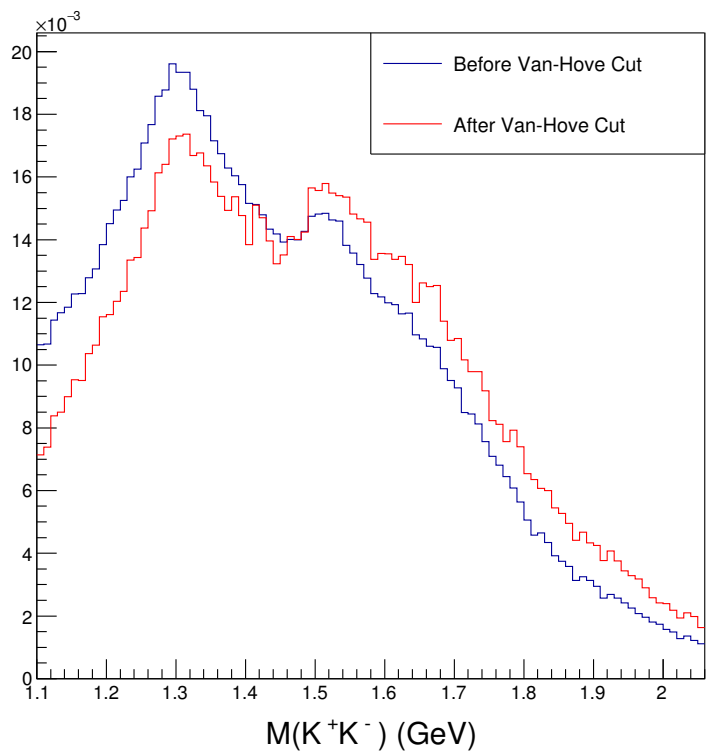

(b) $M\left(K^{+} K^{-}\right)$comparison before and after Van Hove sector cut.

Figure 5.7: Effects of Van Hove sector cut on $M\left(p K^{-}\right)$and $M\left(K^{+} K^{-}\right)$. Plots are normalized so that the sum over all bins is 1 .

nonbaryonic contributions. The intensity function with this added term is

$$
I(\theta, \phi ; \mathbf{V}, B)=\sum_{\substack{\epsilon \epsilon^{\prime} \\ b b^{\prime}}} A_{\epsilon b} V_{\epsilon b} \rho_{\epsilon \epsilon^{\prime}} V_{\epsilon^{\prime} b^{\prime}}^{*} A_{b^{\prime}}^{*}+B^{2} .
$$

\subsubsection{Wave Selection}

In theory, an infinite set of waves are required to form a complete basis. However, with finite statistics, only a finite set of waves can be chosen, and consequently, the series in Eq. 5.66 is truncated. Many fits with various combinations of waves were conducted: first, waves with $J$ up to $11 / 2$ were included. Many of these waves, particularly those with large $J$, had negligible contribution. In addition, as more waves were included, more computation time is required for a fit and it was less likely a successful fit would be obtained. The mass region with $1.47 \mathrm{GeV}<M\left(p K^{-}\right)<1.60 \mathrm{GeV}$ was binned into $10 \mathrm{MeV}$ mass bins. The set of waves used for this region were all the $J=1 / 2$ waves and $J=3 / 2 P$ waves. The mass 
region with $1.60 \mathrm{GeV}<M\left(p K^{-}\right)<1.95 \mathrm{GeV}$ was also binned into $10 \mathrm{MeV}$ mass bins. The set of waves used for this region included all the $J=1 / 2, J=3 / 2$, and $J=5 / 2 D$ waves. Finally, the mass region with $1.95 \mathrm{GeV}<M\left(p K^{-}\right)<2.19 \mathrm{GeV}$ was binned into $15 \mathrm{MeV}$ bins and included the same set of waves in the previous region. For all three regions, the flat, isotropic background term was included. Table 5.1 shows the waves that were used in each mass region.

\subsubsection{Partial Wave Yields}

After the maximization of the likelihood function and the parameters $\mathbf{V}$ and $\mathcal{N}$ from Eq. 5.54 are obtained, the yields for each wave were also obtained. The value $\mathcal{N}$ is the acceptance-corrected total yield obtained from Eq. 5.62. To obtain the yield from a particular wave, first define

$$
\Psi_{b, b^{\prime}}^{\epsilon, \epsilon^{\prime}}=\frac{1}{N^{\text {gen }}} \sum_{i=1}^{N^{\text {gen }}} A_{\epsilon b} \rho_{\epsilon \epsilon^{\prime}} A_{\epsilon^{\prime} b^{\prime}}^{*} .
$$

$\Psi$ is called the raw normalization integral. Substituting this quantity into Eq. 5.62, it follows that

$$
\mathcal{N}=\sum_{\substack{\epsilon \epsilon^{\prime} \\ b b^{\prime}}} V_{\epsilon b} V_{\epsilon^{\prime} b^{\prime}}^{*} \Psi_{b, b^{\prime}}^{\epsilon, \epsilon^{\prime}}+B^{2} .
$$

The yield for a partial wave with quantum numbers $b$ and reflectivity $\epsilon$ is defined to be

$$
\begin{aligned}
\mathcal{N}_{\epsilon b} & =V_{\epsilon b} V_{\epsilon b}^{*} \Psi_{b, b}^{\epsilon, \epsilon}, \\
& =\left|V_{\epsilon b}\right|^{2} \Psi_{b, b}^{\epsilon, \epsilon} .
\end{aligned}
$$

\subsubsection{Statistical Uncertainties}

After the likelihood function is maximized and the parameters are obtained, MINUIT also produces a covariance matrix consisting of the covariances between the real and imaginary components of $\mathbf{V}$. This covariance matrix is used to propagate to the uncertainties in the yields given by Eqs. 5.68 and 5.70. For simplicity, consolidate all the wave numbers $b$ and $\epsilon$ into one index, $\alpha$. Then rewrite $V_{\alpha}$ as $v_{\alpha, R}+i v_{\alpha, I}$, where $v_{\alpha, R}$ denotes the real part of 


\begin{tabular}{|l|l|}
\hline Wave $(J, M, \epsilon, L)$ & $M\left(p K^{-}\right)$Region \\
\hline$(1 / 2,1 / 2,+1, S)$ & $1.47-2.19 \mathrm{GeV}$ \\
$(1 / 2,1 / 2,-1, S)$ & \\
$(1 / 2,1 / 2,+1, P)$ & \\
$(1 / 2,1 / 2,-1, P)$ & \\
$(3 / 2,1 / 2,+1, P)$ & \\
$(3 / 2,1 / 2,-1, P)$ & \\
$(3 / 2,3 / 2,+1, P)$ & \\
$(3 / 2,3 / 2,-1, P)$ & \\
\hline$(3 / 2,1 / 2,+1, D)$ & $1.60-2.19 \mathrm{GeV}$ \\
$(3 / 2,1 / 2,-1, D)$ & \\
$(3 / 2,3 / 2,+1, D)$ & \\
$(3 / 2,3 / 2,-1, D)$ & \\
$(5 / 2,1 / 2,+1, D)$ & \\
$(5 / 2,1 / 2,-1, D)$ & \\
$(5 / 2,3 / 2,+1, D)$ & \\
$(5 / 2,3 / 2,-1, D)$ & \\
\hline Background & \\
\hline
\end{tabular}

Table 5.1: List of waves used in PWA. 
$V_{\alpha}$ and $v_{\alpha, I}$ denotes its imaginary part. It follows that Eqs. 5.68 and 5.70 can be rewritten as

$$
\begin{aligned}
\mathcal{N} & =\sum_{\alpha, \alpha^{\prime}}^{n}\left(v_{\alpha, R}+i v_{\alpha, I}\right)\left(v_{\alpha^{\prime}, R}-i v_{\alpha^{\prime}, I}\right) \Psi_{\alpha, \alpha^{\prime}}, \\
\mathcal{N}_{\alpha} & =\left(v_{\alpha, R}^{2}+v_{\alpha, I}^{2}\right) \Psi_{\alpha, \alpha},
\end{aligned}
$$

respectively, where $n$ is the total number of partial waves used in the fit. The uncertainty for $\mathcal{N}_{\alpha}$ in Eq. 5.72 is given by

$$
\begin{aligned}
\sigma_{\text {stat }}^{2}\left(N_{\alpha}\right)= & \left(\frac{\partial \mathcal{N}_{\alpha}}{\partial v_{\alpha, R}}\right)^{2} \sigma_{\text {stat }}^{2}\left(v_{\alpha, R}\right)+\left(\frac{\partial \mathcal{N}_{\alpha}}{\partial v_{\alpha, I}}\right)^{2} \sigma_{\text {stat }}^{2}\left(v_{\alpha, I}\right) \\
& +2 \frac{\partial \mathcal{N}_{\alpha}}{\partial v_{\alpha, R}} \frac{\partial \mathcal{N}_{\alpha}}{\partial v_{\alpha, I}} \sigma_{\text {stat }}\left(v_{\alpha, R}, v_{\alpha, I}\right) \\
=4 & \Psi_{\alpha, \alpha}\left(v_{\alpha, R}^{2} \sigma_{\text {stat }}^{2}\left(v_{\alpha, R}\right)+v_{\alpha, I}^{2} \sigma_{\text {stat }}^{2}\left(v_{\alpha, I}\right)\right. \\
& \left.+2 v_{\alpha, R} v_{\alpha, I} \sigma_{\text {stat }}\left(v_{\alpha, R}, v_{\alpha, I}\right)\right)
\end{aligned}
$$

The uncertainty for $\mathcal{N}$ in Eq. 5.71 is more complicated. Taking the partial derivatives with respect to $v_{\beta, R}$ of $\mathcal{N}$ in Eq. 5.71 yields

$$
\begin{aligned}
& \frac{\partial \mathcal{N}}{\partial v_{\beta, R}}=\sum_{\alpha, \alpha^{\prime}} {\left[\left(v_{\alpha^{\prime}, R}-i v_{\alpha^{\prime}, I}\right) \Psi_{\alpha, \alpha^{\prime}} \frac{\partial}{\partial v_{\beta, R}}\left(v_{\alpha, R}+i v_{\alpha, I}\right)\right.} \\
&\left.+\left(v_{\alpha, R}+i v_{\alpha, I}\right) \Psi_{\alpha, \alpha^{\prime}} \frac{\partial}{\partial v_{\beta, R}}\left(v_{\alpha^{\prime}, R}-i v_{\alpha^{\prime}, I}\right)\right] .
\end{aligned}
$$

Since

$$
\frac{\partial}{\partial v_{\beta, R}}\left(v_{\alpha, R}+i v_{\alpha, I}\right)=\frac{\partial v_{\alpha, R}}{\partial v_{\beta, R}}=\delta_{\alpha \beta}
$$

where $\delta$ is the Kronecker delta, it follows that

$$
\begin{aligned}
\frac{\partial \mathcal{N}}{\partial v_{\beta, R}} & =\sum_{\alpha, \alpha^{\prime}}\left[\left(v_{\alpha^{\prime}, R}-i v_{\alpha^{\prime}, I}\right) \Psi_{\alpha, \alpha^{\prime}} \delta_{\alpha \beta}+\left(v_{\alpha, R}+i v_{\alpha, I}\right) \Psi_{\alpha, \alpha^{\prime}} \delta_{\alpha^{\prime} \beta}\right] \\
& =\sum_{\alpha^{\prime}}\left(v_{\alpha^{\prime}, R}-i v_{\alpha^{\prime}, I}\right) \Psi_{\beta, \alpha^{\prime}}+\sum_{\alpha}\left(v_{\alpha, R}+i v_{\alpha, I}\right) \Psi_{\alpha, \beta} .
\end{aligned}
$$


Also, since $\rho$ in Eq. 5.67 is symmetric with respect to exchanges in the primed and unprimed indices, it follows that $\Psi$ also has this property. Utilizing this property and relabeling indices, it follows that

$$
\frac{\partial \mathcal{N}}{\partial v_{\beta, R}}=\sum_{\alpha} 2 v_{\alpha, R} \Psi_{\alpha, \beta}
$$

Following a similar computation,

$$
\frac{\partial \mathcal{N}}{\partial v_{\beta, I}}=\sum_{\alpha} 2 v_{\alpha, I} \Psi_{\alpha, \beta} .
$$

Defining

$$
\mathcal{J}=\left[\begin{array}{lllll}
\frac{\partial \mathcal{N}}{\partial v_{1, R}} & \frac{\partial \mathcal{N}}{\partial v_{1, I}} & \cdots & \frac{\partial \mathcal{N}}{\partial v_{n, R}} & \frac{\partial \mathcal{N}}{\partial v_{n, I}}
\end{array}\right]
$$

the uncertainty in $\mathcal{N}$ is given by

$$
\sigma_{\text {stat }}^{2}(\mathcal{N})=\mathcal{J} \mathcal{C} \mathcal{J}^{T}
$$

where $\mathcal{C}$ is the covariance matrix given by MINUIT.

\subsection{Fit Results}

Many fits were conducted to try to obtain the best wave combination. However, the fitting procedure is computationally expensive and not every combination can be tried. The results shown are for the procedures described in Sections 5.4 and 5.5 , though many other combinations were attempted (c.f. Section 5.8). Figures 5.8 to 5.11 show the acceptance corrected yields for each wave used in the fit as a function of the $p K^{-}$invariant mass. Figures 5.12 and 5.13 show the total acceptance corrected yield $(\mathcal{N})$ and the background yield, respectively. 


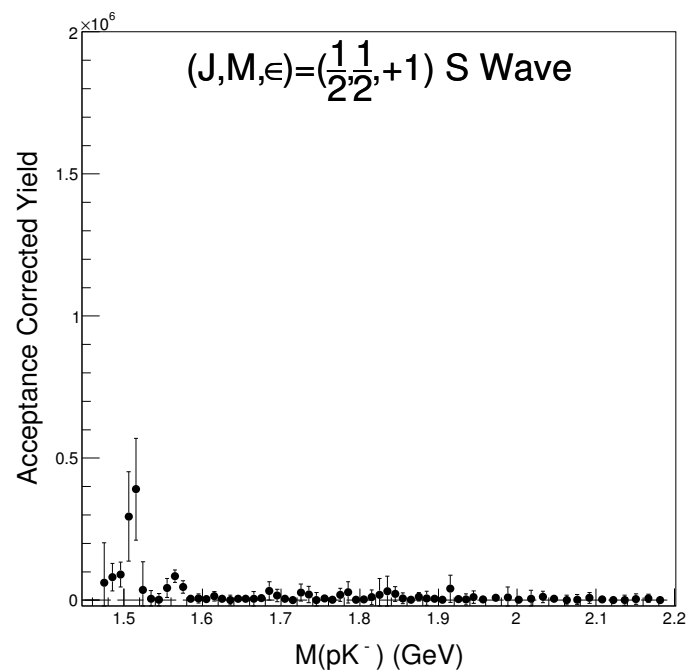

(a) $(J, M, \epsilon)=(1 / 2,1 / 2,+1) S$ wave acceptance corrected yield.

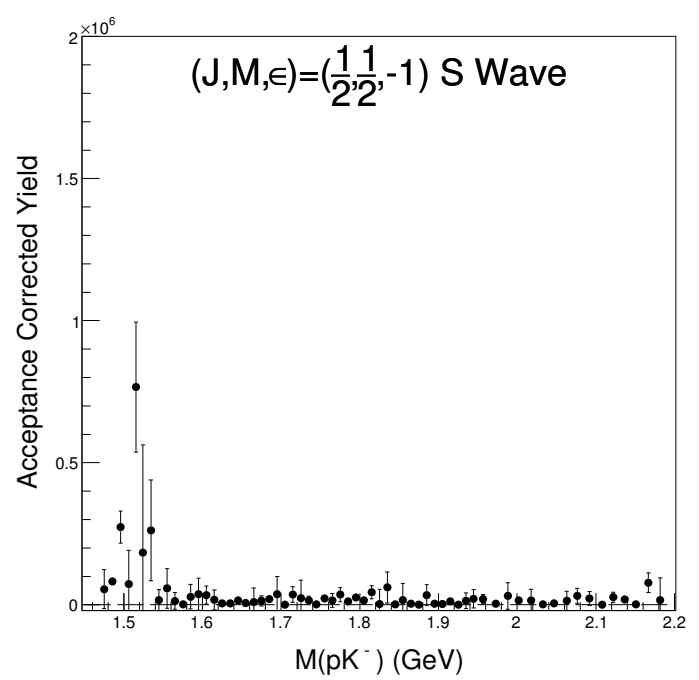

(c) $(J, M, \epsilon)=(1 / 2,1 / 2,-1) S$ wave acceptance corrected yield.

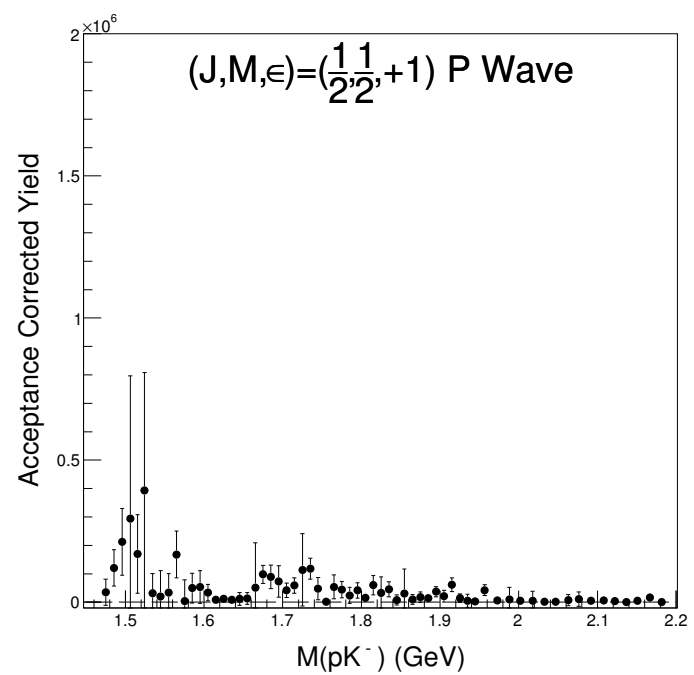

(b) $(J, M, \epsilon)=(1 / 2,1 / 2,+1) P$ wave acceptance corrected yield.

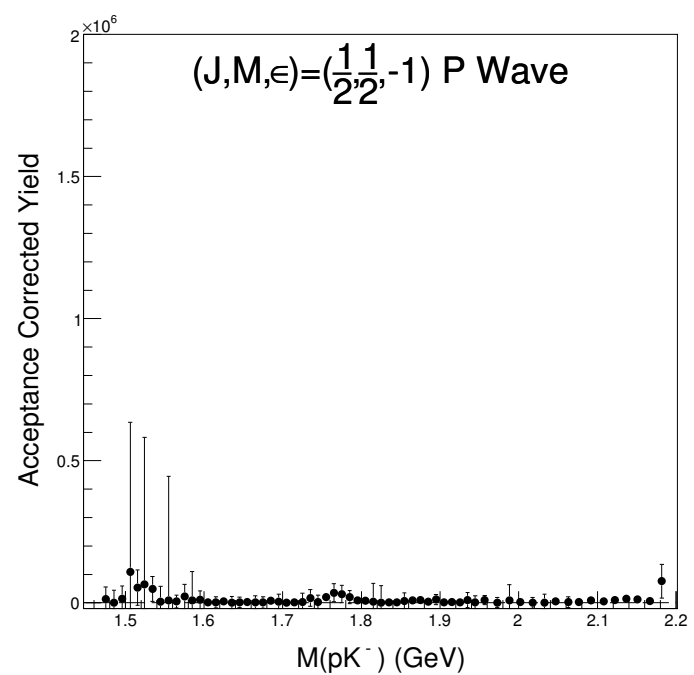

(d) $(J, M, \epsilon)=(1 / 2,1 / 2,-1) P$ wave acceptance corrected yield.

Figure 5.8: $J=1 / 2$ waves acceptance corrected yields. 


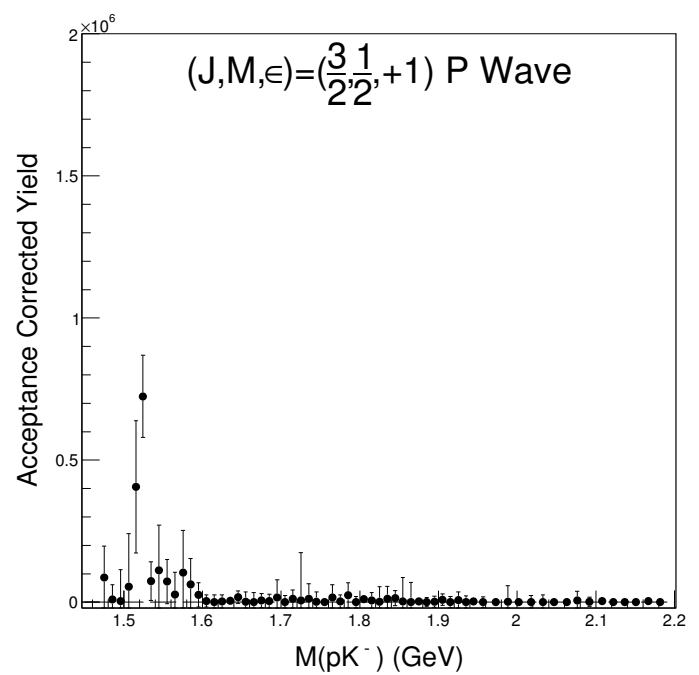

(a) $(J, M, \epsilon)=(3 / 2,1 / 2,+1) P$ wave acceptance corrected yield.

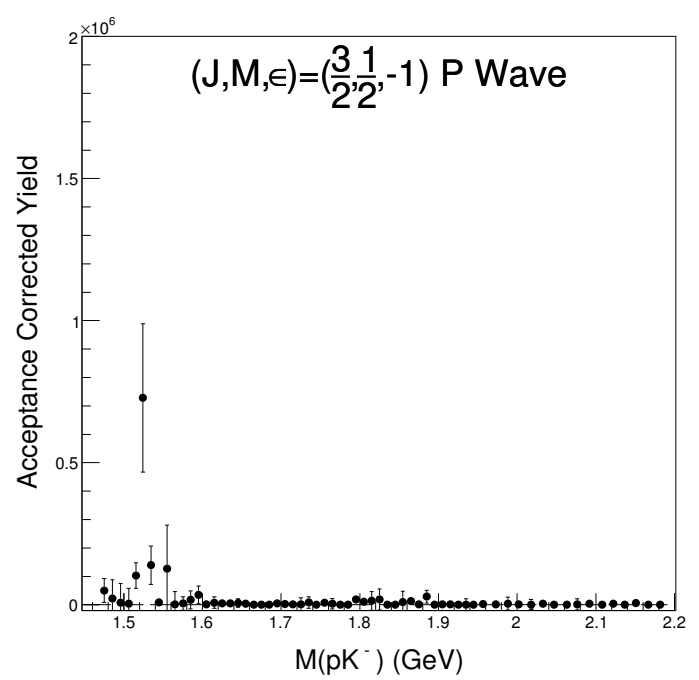

(c) $(J, M, \epsilon)=(3 / 2,1 / 2,-1) P$ wave acceptance corrected yield.

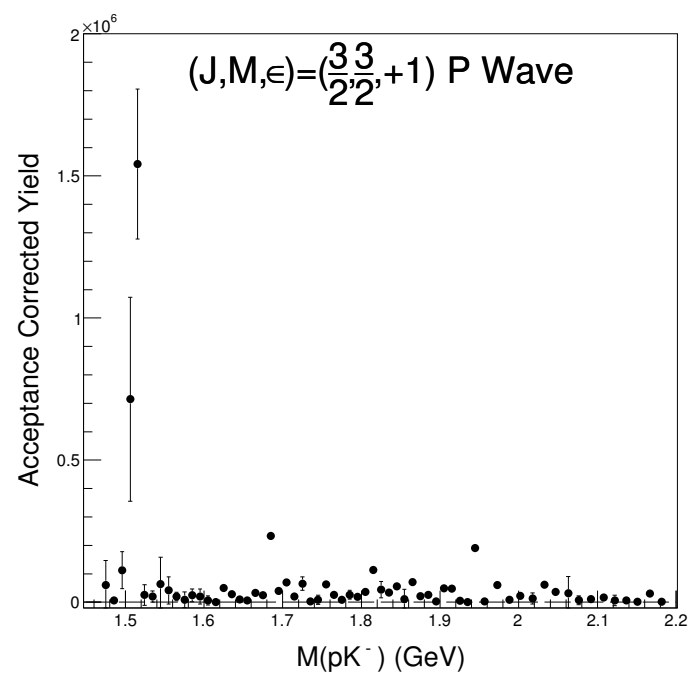

(b) $(J, M, \epsilon)=(3 / 2,3 / 2,+1) P$ wave acceptance corrected yield.

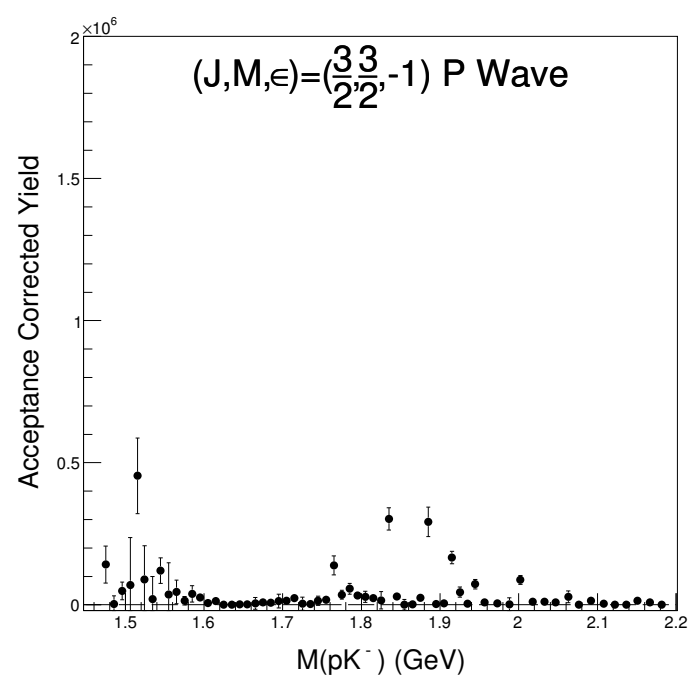

(d) $(J, M, \epsilon)=(3 / 2,3 / 2,-1) P$ wave acceptance corrected yield.

Figure 5.9: $\quad J=3 / 2 P$ waves acceptance corrected yields. 


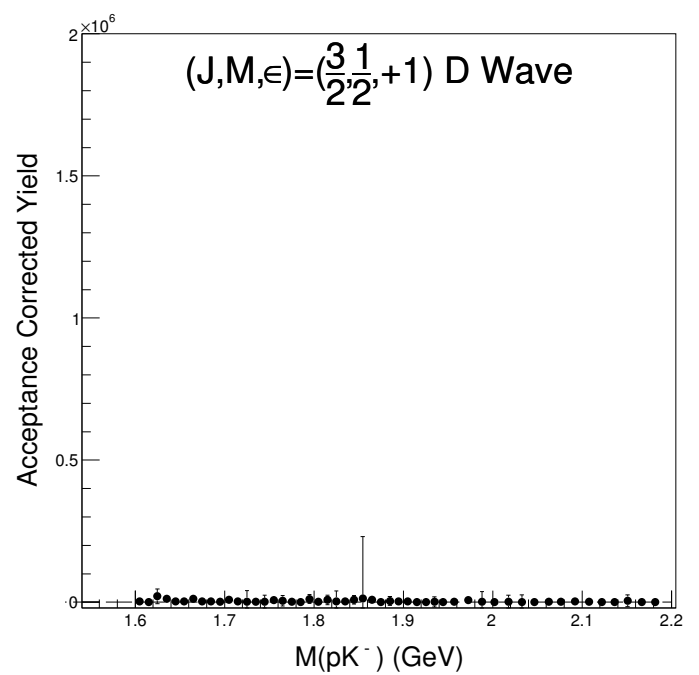

(a) $(J, M, \epsilon)=(3 / 2,1 / 2,+1) D$ wave acceptance corrected yield.

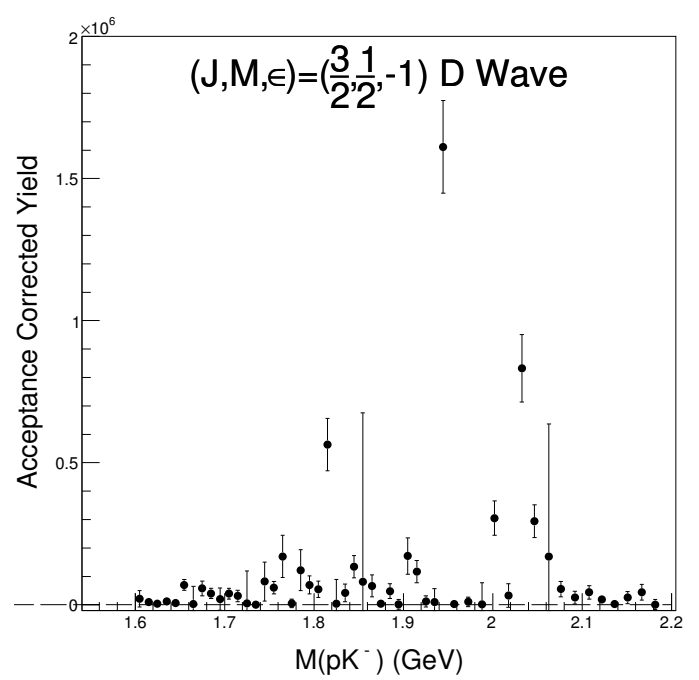

(c) $(J, M, \epsilon)=(3 / 2,1 / 2,-1) D$ wave acceptance corrected yield.

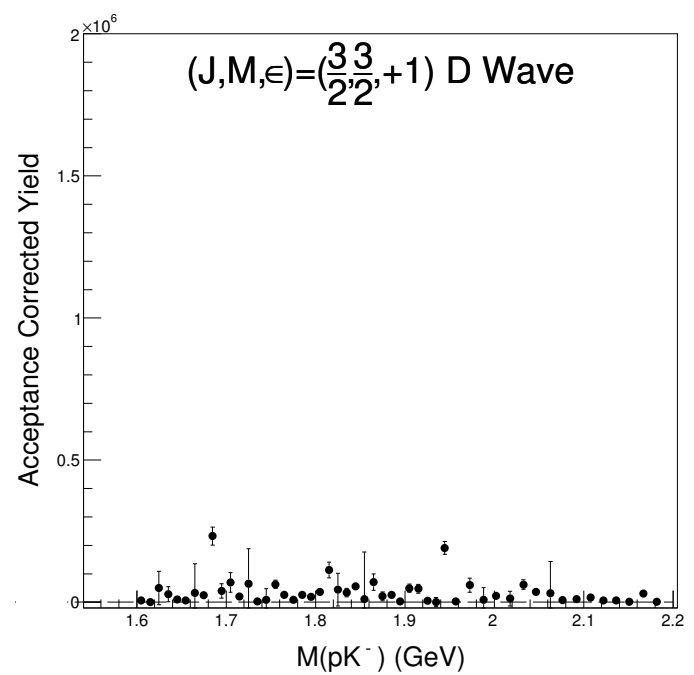

(b) $(J, M, \epsilon)=(3 / 2,3 / 2,+1) D$ wave acceptance corrected yield.

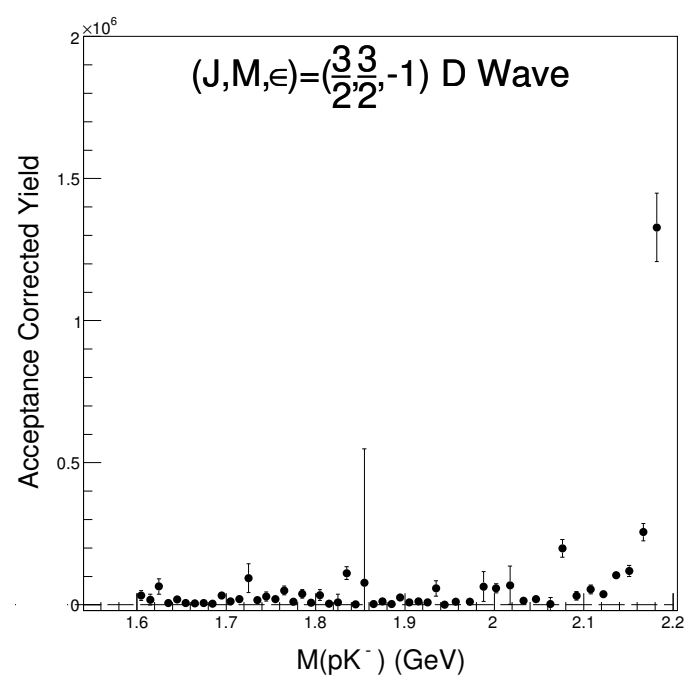

(d) $(J, M, \epsilon)=(3 / 2,3 / 2,-1) D$ wave acceptance corrected yield.

Figure 5.10: $J=3 / 2 D$ waves acceptance corrected yields. 


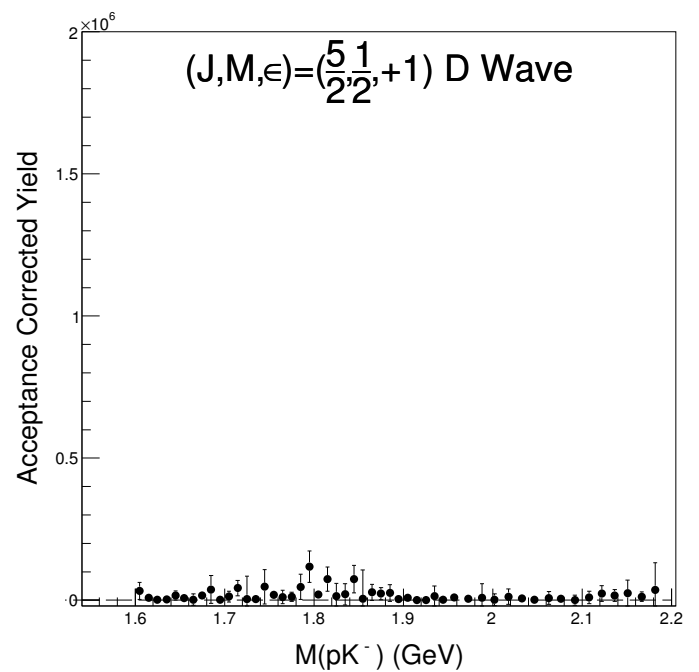

(a) $(J, M, \epsilon)=(5 / 2,1 / 2,+1) D$ wave acceptance corrected yield.

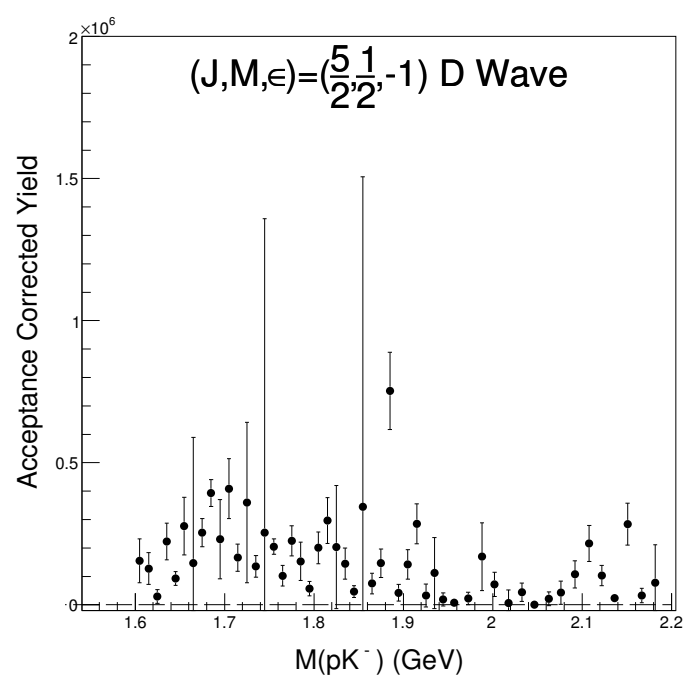

(c) $(J, M, \epsilon)=(5 / 2,1 / 2,-1) D$ wave acceptance corrected yield.

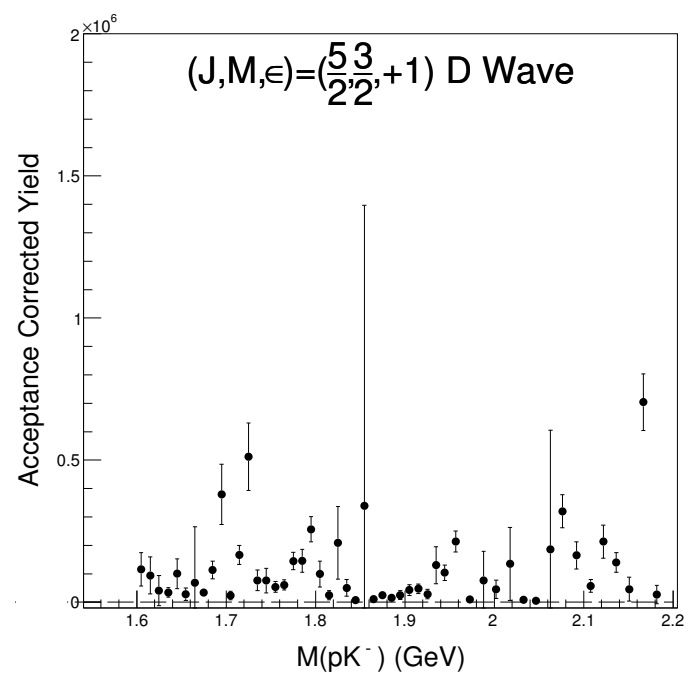

(b) $(J, M, \epsilon)=(5 / 2,3 / 2,+1) D$ wave acceptance corrected yield.

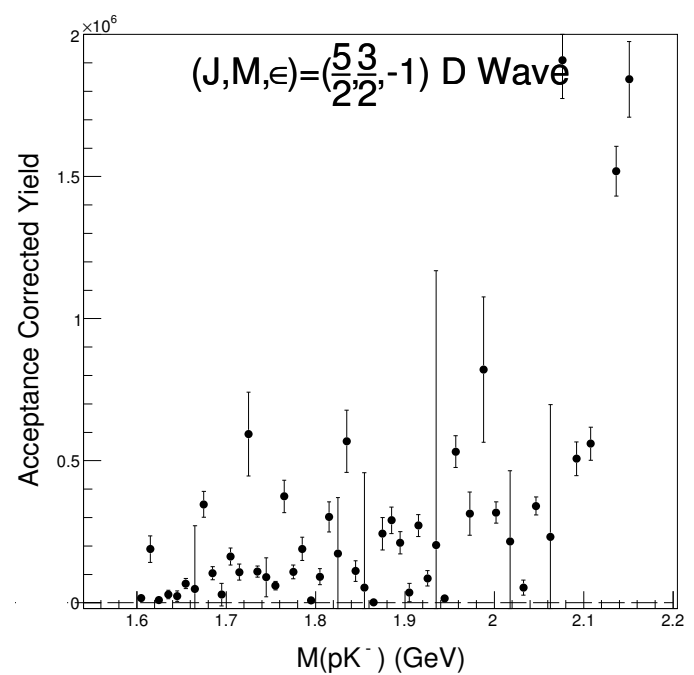

(d) $(J, M, \epsilon)=(5 / 2,3 / 2,-1) D$ wave acceptance corrected yield.

Figure 5.11: $J=5 / 2 D$ waves acceptance corrected yields. 


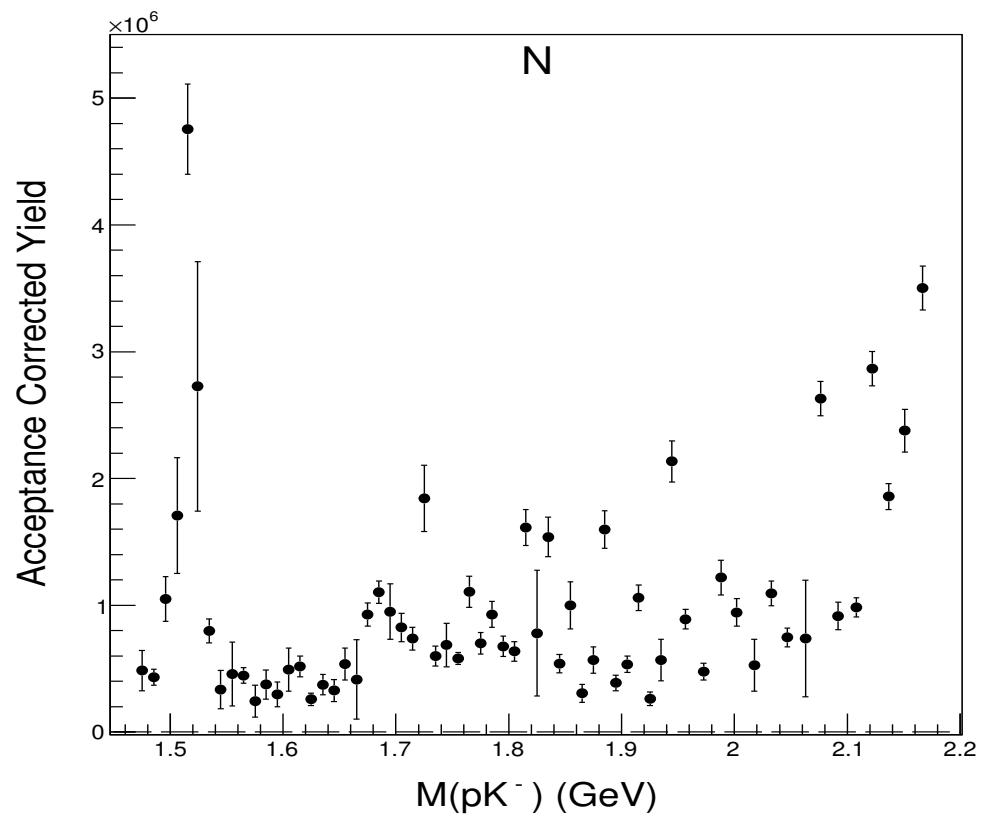

Figure 5.12: $\mathcal{N}$ acceptance corrected yield.

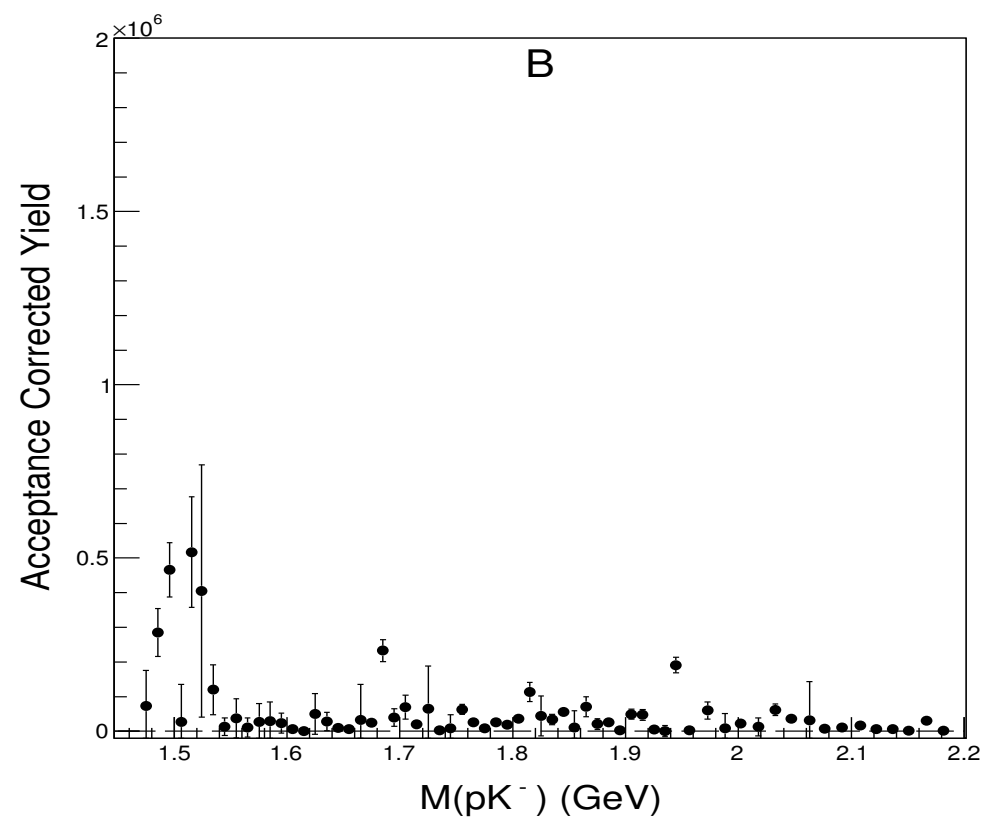

Figure 5.13: Background wave acceptance corrected yield. 


\subsection{Fit Quality}

After the amplitudes were attained, the generated events were weighted according to the intensity using the fitted parameters. The distributions generated after weighting are the fit-predicted distributions and these were compared to the actual data. As shown in Fig. 5.14, the fits for the $1.47<M\left(p K^{-}\right)<1.6 \mathrm{GeV}$ region were in acceptable agreement. However, the fits in the mass region with $M\left(p K^{-}\right)>1.6 \mathrm{GeV}$ were not successful. Many fits were conducted in order to obtain an acceptable fit in this region. Different combinations of waves, binning schemes, and event selections were attempted, but no method yielded a successful fit in that region. This was most likely due to meson contributions still being significant, contrary to the model's assumption that only hyperons are produced in the reaction. 


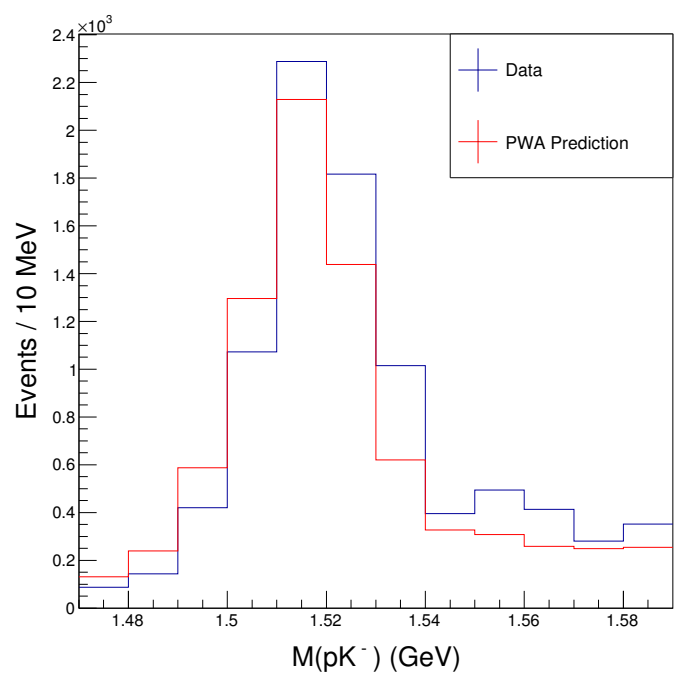

(a) Comparison of $M\left(p K^{-}\right)$distribution between PWA prediction and data.

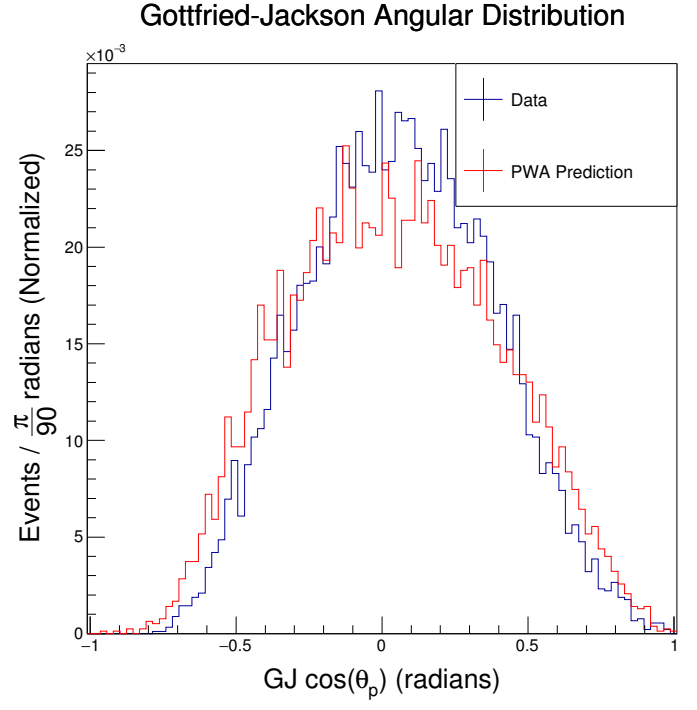

(b) Comparison of proton $\cos (\theta)$ distribution in the GJ frame between PWA prediction and data.

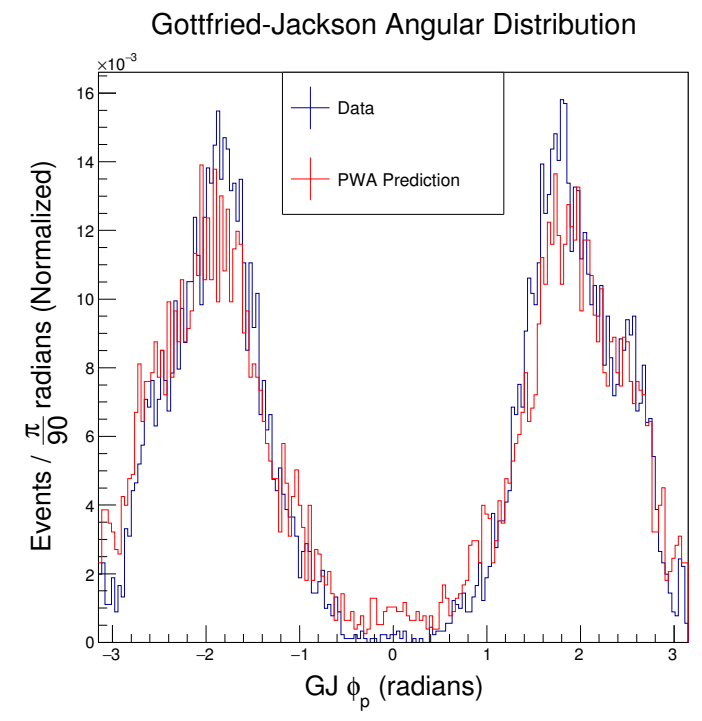

(c) Comparison of proton $\phi$ distribution in the GJ frame between PWA prediction and data.

Figure 5.14: Comparison between PWA predicted distributions and data in the $1.47<$ $M\left(p K^{-}\right)<1.6 \mathrm{GeV}$ mass region. 


\subsection{Conclusions}

As shown in Sections 5.6 and 5.7, the best fit results were in the $\Lambda(1520)$ region, where there are the most statistics. Not surprisingly, the dominant wave in that region is the $J=3 / 2$, which is in agreement with previous measurements [7]. A result of this fit is that among the $M_{j}$ quantum numbers, the $M_{j}=3 / 2$ wave contributions dominate over the $M_{j}=1 / 2$ wave. This can perhaps provide information on the exchange particle that produces the $\Lambda(1520)$. However, this should be taken with some degree of skepticism. One of the possible negative effects of a partial wave fit is that the solution is not unique: different combinations of waves can yield the same result. In addition, while performing the fits, it was found that the results depend heavily on the choice of waves included. This is not surprising as removing one wave is equivalent to setting its amplitude to 0. If this wave were significant in one fit, another wave (or waves) must take its place.

Many different combinations of waves and simulation schemes were attempted for this work. Fits using waves with $J$ up to $11 / 2$ were attempted. Also, an isotropic simulation ( $t$ slope is 0$)$ was attempted. In addition, the $t^{\prime}$ cut was varied and the smallest value attempted was half a standard deviation. However, with this cut, only $16 \%$ of the data remain and most of the signal in the higher mass regions was lost. While the Van Hove sector cut improved the signal for the $\Lambda(1520)$, it also removed signal in the higher mass regions. The results for each combination in the $M\left(p K^{-}\right)>1.6 \mathrm{GeV}$ region were the same however: the fits did not accurately describe the data.

Furthermore, the model used had a key simplifying assumption: it only assumes the production of an excited hyperon. In reality, the production of a meson decaying into $K^{+} K^{-}$is a significant background. This background was suppressed by applying the small momentum transfer and Van Hove sector cuts, but at the cost of losing signal in the $M\left(p K^{-}\right)>1.6 \mathrm{GeV}$ region. Even so, the background persisted even after the cuts. The fit quality in the $M\left(p K^{-}\right)>1.6 \mathrm{GeV}$ region is poor and may be due to the inadequate model used. An improvement of the model would be to consider the production of both the mesons and baryons in the reaction. A model such as the one described has been the 
topic of recent theoretical work in, for example, reference [73]. This information is also of importance for those studying meson spectroscopy. As shown in Fig. $3.14 \mathrm{~d}$, there are a lot of baryon contributions that would be difficult to remove, in analogy to trying to remove the meson contributions. With a complete model that considers both meson and baryon contributions, a full partial wave fit can be conducted. 


\section{CHAPTER 6}

\section{Conclusion}

The photoproduction of mesons is perpetually being used for the investigation of intermediate resonant states. This is fundamental in the understanding of the nonperturbative region of QCD. This work focused on the reactions $\gamma p \rightarrow p K^{+} K^{-}$and $\gamma p \rightarrow p \pi^{+} \pi^{-}$utilizing the g12 data set collected with the CLAS detector that is housed in Hall B at Jefferson Lab.

For $\gamma p \rightarrow p K^{+} K^{-}$, a partial wave analysis was performed for the $p K^{-}$system as a function of $M\left(p K^{-}\right)$in an exploratory search for excited hyperons. The model used, its formalism, and the fit procedures were described in Chapter 5. The results of the PWA concluded that the fit was acceptable in the region containing the $\Lambda(1520)$. However, for the mass region $M\left(p K^{-}\right)>1.6 \mathrm{GeV}$, the fits were not successful. This was most likely due to meson contributions not being suppressed enough and a model that does not adequately describe hyperon production in the presence of background mesonic contributions. Going forward, a reaction model that considers both possible processes is needed. By analogy, this should also hold true for meson spectroscopy in $\gamma p \rightarrow p X, X \rightarrow K^{+} K^{-}$. Although the PWA attempt for the high mass $Y^{*}$ did not yield reliable results, it demonstrated the necessity of understanding and modeling of the background process in this type of PWA. When both meson and hyperon processes contribute, it is clear that the amplitudes of both processes is needed and both channels should be fit simultaneously.

In addition, for both of the reactions, a measurement of the polarization observable $I^{\odot}$ was presented. The measurement of $I^{\odot}$ was conducted for the first time for $\gamma p \rightarrow$ $p K^{+} K^{-}$and for $\gamma p \rightarrow p \pi^{+} \pi^{-}$in the $W>2.3 \mathrm{GeV}$ region. Polarization observables, such as $I^{\odot}$, are necessary to constrain the parameters of the reaction models and achieve a better understanding of the reaction mechanisms. Several features of the asymmetry were described in Chapter 4 . Perhaps the most striking feature of the asymmetry was the apparent agreement (up to a sign) of the leading coefficient of a Fourier sine fit among three different plane and angle definitions. Also, a comparison of the asymmetry for $\gamma p \rightarrow$ 
$p \pi^{+} \pi^{-}$and $\gamma p \rightarrow p K^{+} K^{-}$was conducted. It was concluded that the asymmetry for $\gamma p \rightarrow$ $p \pi^{+} \pi^{-}$as a function of the angle $\phi$ defined in Chapter 4 is dominated by $\sin (2 \phi)$, whereas for $\gamma p \rightarrow p K^{+} K^{-}$it is dominated by $\sin (\phi)$ when it is decomposed into its Fourier sine series. The two-pion results at $W>2.3 \mathrm{GeV}$ will also become important in extracting the properties of intermediate nucleon resonances [58]. In order to fully make sense of the physics behind these results, a better theoretical understanding of the reactions is needed. These results generated a lot of interest from the theoretical community and will certainly aid in the development of theoretical models for double kaon photoproduction. 
Bibliography

[1] W. Heisenberg. "On the structure of atomic nuclei". Z. Phys. 77 (1932), pp. 1-11. DOI: $10.1007 / \mathrm{BF} 01342433$.

[2] A. Pais. "Some Remarks on the V-Particles". Phys. Rev. 86 (1952), pp. 663-672. DoI: $10.1103 /$ PhysRev.86.663.

[3] M. Gell-Mann. "A Schematic Model Of Baryons and Mesons". Phys.Lett. 8 (1964), pp. 214-215.

[4] T. Nakano and K. Nishijima. "Charge Independence for V-particles". Prog. Theor. Phys. 10 (1953), pp. 581-582. DOI: 10.1143/PTP.10.581.

[5] David J. Gross and Frank Wilczek. "Ultraviolet Behavior of Non-Abelian Gauge Theories". Phys. Rev. Lett. 30 (26 1973), pp. 1343-1346. Dor: 10.1103 / PhysRevLett. 30.1343, URL: http:// link.aps.org/doi/10.1103/PhysRevLett. 30 . 1343 .

[6] H. David Politzer. "Reliable Perturbative Results for Strong Interactions?" Phys. Rev. Lett. 30 (26 1973), pp. 1346-1349. DOI: 10.1103/PhysRevLett. 30.1346 . URL: http://link.aps.org/doi/10.1103/PhysRevLett.30.1346.

[7] K. A. Olive, Particle Data Group, et al. "Review of Particle Physics". Chin. Phys. C38 (2014), p. 090001. DOI: 10.1088/1674-1137/38/9/090001.

[8] N. Beaudoin, G. Landry, and R. Sandapen. "Generalized isospin, generalized mass groups, and generalized Gell-Mann-Okubo formalism" (2013). arXiv: 1309.0517 [hep-ph].

[9] Simon Capstick and W. Roberts. "Strange decays of nonstrange baryons". Phys. Rev. D 58 (7 1998), p. 074011. DOI: 10.1103 / PhysRevD . 58.074011 . URL: http: //link.aps.org/doi/10.1103/PhysRevD.58.074011.

[10] Simon Capstick and Nathan Isgur. "Baryons in a relativized quark model with chromodynamics". Phys. Rev. D 34 (9 1986), pp. 2809-2835. DoI: 10.1103 /PhysRevD. 34.2809, URL: http://link.aps.org/doi/10.1103/PhysRevD.34.2809.

[11] B. Krusche and S. Schadmand. "Study of nonstrange baryon resonances with meson photoproduction". Prog. Part. Nucl. Phys. 51 (2003), pp. 399-485. DOI: $10.1016 /$ S0146-6410(03)90005-6, arXiv: nucl-ex/0306023 [nucl-ex].

[12] D. B. Lichtenberg et al. "Baryon Masses in a Relativistic Quark-Diquark Model". Phys. Rev. Lett. 48 (24 1982), pp. 1653-1656. Dor: 10.1103 / PhysRevLett . 48 . 1653. URL: http://link.aps.org/doi/10.1103/PhysRevLett.48.1653.

[13] J. Ferretti, A. Vassallo, and E. Santopinto. "Relativistic quark-diquark model of baryons". Phys. Rev. C 83 (6 2011), p. 065204. DoI: 10.1103 / PhysRevC. 83 . 065204. URL: http://link.aps.org/doi/10.1103/PhysRevC.83.065204. 
[14] E. Santopinto and J. Ferretti. "Strange and nonstrange baryon spectra in the relativistic interacting quark-diquark model with a Gürsey and Radicati-inspired exchange interaction". Phys. Rev. C 92 (2 2015), p. 025202. DoI: 10.1103/PhysRevC.92. 025202, URL: http://link.aps.org/doi/10.1103/PhysRevC.92.025202,

[15] Robert G. Edwards et al. "Flavor structure of the excited baryon spectra from lattice QCD". Phys. Rev. D 87 (5 2013), p. 054506. DoI:10.1103/PhysRevD . 87.054506. URL: http://link.aps.org/doi/10.1103/PhysRevD.87.054506.

[16] R. P. Feynman. "Space-Time Approach to Quantum Electrodynamics". Phys. Rev. 76 (6 1949), pp. 769-789. DOI: 10.1103 /PhysRev.76.769, URL: http:// link . aps.org/doi/10.1103/PhysRev.76.769.

[17] W. Roberts and T. Oed. "Polarization observables for two-pion production off the nucleon". Phys. Rev. C71 (2005), p. 055201. Dor: 10.1103 /PhysRevC. 71.055201. arXiv: nucl-th/0410012 [nucl-th].

[18] C. Amsler et al. "Review of Particle Physics". Physics Letters B 667.1?5 (2008). Review of Particle Physics, pp. 1 -6. ISSN: 0370-2693. DOI: http://dx.doi.org/ $10.1016 / j \cdot$ physletb.2008.07.018. URL: http://wWw.sciencedirect. com/science/article/pii/s0370269308008435.

[19] Suh Urk Chung. "SPIN FORMALISMS". CERN-71-08 (1971).

[20] Suh Urk Chung. "Formulas for Partial Wave Analysis". BNL-QGS-93-05 (1995).

[21] Carlos W. Salgado and Dennis P. Weygand. "On the Partial-Wave Analysis of Mesonic Resonances Decaying to Multiparticle Final States Produced by Polarized Photons". Phys.Rept. 537 (2014), pp. 1-58. Dor: $10.1016 /$ j.physrep.2013.11.005, arXiv: 1310.7498 [nucl-ex].

[22] J. Hamilton and B. Tromberg. Partial Wave Amplitudes and Resonance Poles. Oxford University Press, Ely House, 1972.

[23] V.N. Gribov. The Theory of Complex Angular Momenta. Cambridge University Press, 2003.

[24] Christoph W. Leemann, David R. Douglas, and Geoffrey A. Krafft. "THE CONTINUOUS ELECTRON BEAM ACCELERATOR FACILITY: CEBAF at the Jefferson Laboratory". Annu. Rev. Nucl. Part. Sci. 51 (2001), pp. 413-450. uRL: http: / / www . annualreviews . org / doi / pdf / 10 . 1146/annurev . nucl . 51 . 101701.132327.

[25] Y. G. Sharabian et al. "A new highly segmented start counter for the CLAS detector". Nucl. Instrum. Meth. A556 (2006), pp. 246-258. DOI: 10.1016/j.nima.2005.10. 031 .

[26] Steve Gagnon. Jefferson Lab Site Tour. http : / / education · jlab . org / sitetour/guidedtour05.1.alt.html. Accessed: 2015-10-19. 
[27] M. C. Kunkel. "Photoproduction of $\pi^{0}$ on hydrogen with CLAS from $1.1 \mathrm{GeV}-5.45$ GeV Using $e^{+} e^{-} \gamma$ Decay". PhD thesis. Old Dominion University, 2014.

[28] Haakon Olsen and L. C. Maximon. "Photon and Electron Polarization in HighEnergy Bremsstrahlung and Pair Production with Screening". Phys. Rev. 114 (3 1959), pp. 887-904. DOI: 10 .1103/PhysRev.114 . 887. URL: http: / / link . aps.org/doi/10.1103/PhysRev.114.887.

[29] D. I. Sober et al. "The bremsstrahlung tagged photon beam in Hall B at JLab". Nucl. Instrum. Meth. A440 (2000), pp. 263-284. DOI: 10.1016/S0168-9002 (99) $00784-6$.

[30] B. A. Mecking et al. "The CEBAF Large Acceptance Spectrometer (CLAS)". Nucl. Instrum. Meth. A503 (2003), pp. 513-553. DOI: 10.1016/S0168-9002 (03)010015.

[31] Jefferson Lab. Jefferson Lab Picture Exchange. https://www.jlab.org/photos. Accessed: 2015-10-19.

[32] Steve Gagnon. Jefferson Lab Site Tour. http : / / education - jlab . org / sitetour/hallbin02.l.html, Accessed: 2015-10-19.

[33] M. D. Mestayer et al. "The CLAS drift chamber system". Nucl. Instrum. Meth. A449 (2000), pp. 81-111. DOI: 10.1016/S0168-9002(00)00151-0.

[34] D. S. Carman et al. "The region one drift chamber for the CLAS spectrometer". Nucl. Instrum. Meth. A419 (1998), pp. 315-319. DOI: 10.1016/S0168-9002 (98) 00805-5.

[35] L. M. Qin et al. "Prototype studies and design considerations for the CLAS region 2 drift chambers". Nucl. Instrum. Meth. A411 (1998), pp. 265-274. DOI: $10.1016 /$ S0168-9002(98)00302-7.

[36] D. Lawrence and M.D. Mestayer. "CLAS Drift Chambers Calibrations: Software and Procedures". CLAS-Note 1999-018 (1999).

[37] J. T. Goetz. " $\Xi$ Hyperon Photoproduction from Threshold to $5.4 \mathrm{GeV}$ with the CEBAF Large Acceptance Spectrometer". PhD thesis. University of California, Los Angeles, 2011.

[38] G. Adams et al. "The CLAS Cherenkov detector". Nucl. Instrum. Meth. A465 (2001), pp. 414-427. DOI: $10.1016 /$ S0168-9002(00) 01313-9.

[39] E. S. Smith et al. "The time-of-flight system for CLAS". Nucl. Instrum. Meth. A432 (1999), pp. 265-298. DOI: 10.1016/S0168-9002 (99)00484-2.

[40] M. Amarian et al. "The CLAS forward electromagnetic calorimeter". Nucl. Instrum. Meth. A460 (2001), pp. 239-265. DOI: 10.1016/S0168-9002(00)00996-7. 
[41] M. Anghinolfi et al. "The CLAS electromagnetic calorimeter at large angles". Nucl. Instrum. Meth. A537 (2005), pp. 562-570. DOI: $10.1016 /$ j.nima.2004.08.070. arXiv: nucl-ex/0403041 [nucl-ex].

[42] Jason Bono. "First Time Measurements of Polarization Observables for the Charged Cascade Hyperon in Photoproduction". PhD thesis. Florida International University, 2014.

[43] E. Pasyuk. "Energy loss corrections for charged particles in CLAS". CLAS-Note 200\%016 (2007).

[44] J. D. Jackson. Classical Electrodynamics. New York, NY: Wiley, 1999.

[45] M. Williams, D. Applegate, and C.A. Meyer. "Determining Momentum and Energy Corrections for g1c Using Kinematic Fitting". CLAS-Note 2004-017 (2004).

[46] L. Demortier and L. Lyons. "Everything you always wanted to know about pulls". $C D F / A N A L / P U B L I C / 5776$ (2002).

[47] A. G. Frodesen and O. Skjeggestad. Probability and Statistics in Particle Physics. 136 South Broadway, Irvington-on-Hudson, New York 10533: Columbia University Press, 1979 .

[48] M. Williams and C.A. Meyer. "Kinematic Fitting in CLAS". CLAS-Note 2003-017 (2003).

[49] S. Strauch et al. "Beam-helicity asymmetries in double-charged-pion photoproduction on the proton". Phys.Rev.Lett. 95 (2005), p. 162003. Dor: 10.1103/PhysRevLett. 95.162003, arXiv: hep-ex/0508002 [hep-ex].

[50] D. Krambrich et al. "Beam-Helicity Asymmetries in Double Pion Photoproduction off the Proton". Phys.Rev.Lett. 103 (2009), p. 052002. DoI: 10.1103 / PhysRevLett. 103.052002, arXiv: 0907.0358 [nucl-ex].

[51] W. Langgartner et al. "Direct observation of a rho decay of the $\mathrm{D}(13)(1520)$ baryon resonance". Phys. Rev. Lett. 87 (2001), p. 052001. Dor: $10.1103 /$ PhysRevLett. 87.052001 .

[52] M. Oberle et al. "Measurement of the beam-helicity asymmetry $I^{\odot}$ in the photoproduction of $\pi^{0} \pi^{ \pm}$pairs off protons and off neutrons". Eur. Phys. J. A50 (2014), p. 54. Dor: $10.1140 /$ epja/i2014-14054-5, arXiv: 1403.1989 [nucl-ex].

[53] J. C. Nacher and E. Oset. "Study of polarization observables in double pion photoproduction on the proton". Nucl. Phys. A697 (2002), pp. 372-387. DOI: 10.1016/ S0375-9474(01)01251-9, arXiv: nucl-th/0106005 [nucl-th].

[54] L. Roca. "Helicity asymmetries in double pion photoproduction on the proton". Nucl. Phys. A748 (2005), pp. 192-205. DOI: 10.1016/j.nuclphysa.2004.10.028. arXiv: nucl-th/0407049 [nucl-th]. 
[55] A. Fix and H. Arenhovel. "Double pion photoproduction on nucleon and deuteron". Eur. Phys. J. A25 (2005), pp. 115-135. DOI: $10.1140 /$ epja/i2005-10067-5. arXiv: nucl-th/0503042 [nucl-th].

[56] M. Oberle et al. "Measurement of the beam-helicity asymmetry $I^{\odot}$ in the photoproduction of $\pi^{0}$-pairs off the proton and off the neutron". Phys. Lett. B721 (2013), pp. 237-243. DOI: $10.1016 / j \cdot$ physletb.2013.03.021. arXiv: 1304.1919 [nucl-ex].

[57] W. Roberts. "Polarization observables in $\gamma N \rightarrow K \bar{K} N$ ". Phys.Rev. C73 (2006), p. 035215. DOI: 10.1103 / PhysRevC.73.035215, arXiv: nucl-th/0412041 [nucl-th].

[58] A. V. Anisovich et al. "Properties of baryon resonances from a multichannel partial wave analysis". The European Physical Journal A 48.2 (2012), pp. 1-13. ISSN: 1434601X. DOI: $10.1140 /$ epja/i2012-12015-8. uRL: http://dx.doi.org/10 . $1140 /$ epja/i2012-12015-8.

[59] A. Freese. Private communications.

[60] M. Jacob and G. C. Wick. "On the general theory of collisions for particles with spin". Annals Phys. 7 (1959). [Annals Phys.281,774(2000)], pp. 404-428. DOI: $10.1016 /$ 0003-4916(59) 90051-X.

[61] Michael A. Morrison and Gregory A. Parker. "A Guide to Rotations in Quantum Mechanics". Aust. J. Phys. 40.4 (July 1987), pp. 465-497. ISSN: 0004-9506. DOI: 10. 1071/PH870465. uRL: http://dx.doi.org/10.1071/PH870465.

[62] M. E. Rose. Elementary Theory of Angular Momentum. Dover Pub., 1995.

[63] A.G. Frodesen, O. Skjeggestad, and H Tøfte. Probability and Statistics in Particle Physics. Columbia University Press, 1979.

[64] Radhakrishna C. Rao. "Information and the accuracy attainable in the estimation of statistical parameters". Bull. Calcutta Math. Soc. 37 (1945), pp. 81-91. uRL: http: //www.ams.org/mathscinet-getitem?mr=0015748.

[65] F. James and M. Roos. "Minuit: A System for Function Minimization and Analysis of the Parameter Errors and Correlations". Comput. Phys. Commun. 10 (1975), pp. 343367. DOI: $10.1016 / 0010-4655$ (75) 90039-9.

[66] F. James. "MINUIT Function Minimization and Error Analysis: Reference Manual Version 94.1" (1994).

[67] J. Pond et al. "A software framework used to perform Partial Wave and Amplitude Analysis (PWA) with the goal of extracting resonance information from multiparticle final states". Presented at the CLAS Collaboration meeting in Newport News, VA., 2015 .

[68] C.W. Salgado et al. "PyPWA: A Partial-Wave/Amplitude Analysis Software Framework". Presented at the Hadron 2015 conference in Newport News, VA., 2015. 
[69] J P Cummings and D P Weygand. An Object-Oriented Approach to Partial Wave Analysis. Tech. rep. physics/0309052. 2003. uRL: http://cds.cern.ch/record/ 641847.

[70] Shigeo Minami. "An Invariance Theorem for Cross Sections of Meson-Nucleon Scattering". Progress of Theoretical Physics 11.2 (1954), pp. 213-218. DOI: 10.1143/ PTP.11.213, eprint: http://ptp.oxfordjournals.org/content/11/2/ 213.full.pdf+html. URL: http://ptp.oxfordjournals.org/content/ $11 / 2 / 213$. abstract.

[71] W. S. C. Williams. An Introduction to Elementary Particles. 111 Fifth Avenue, New York, New York 10003: Academic Press, 1971.

[72] L. Van Hove. "Final state classification and new phase space plot for many body hadron collisions". Phys. Lett. B28 (1968), pp. 429-431. DOI: 10 . 1016/0370$2693(69) 90343-8$.

[73] M. Shi et al. "Double-Regge exchange limit for the $\gamma p \rightarrow K^{+} K^{-} p$ reaction". Phys. Rev. D 91 (3 2015), p. 034007. Dor: 10.1103/PhysRevD.91.034007. uRL: http: //link.aps.org/doi/10.1103/PhysRevD.91.034007.

[74] V. Devanathan. Angular Momentum Techniques in Quantum Mechanics. Kluwer Academic Publishers, 2002. 
Appendices 


\section{Wigner $D$ and $d$ Matrices}

These are relevant formulas involving Wigner $D$ and $d$ matrices that are used in the formalism of the PWA (Chapter 5) obtained from references [7, 19 21, 61, 62, 74. The notation for the Clebsch-Gordan coefficients used is

$$
\left(j_{1} m_{1} j_{2} m_{2} \mid j m\right)
$$

where

$$
\begin{aligned}
\left|j_{1} m_{1}\right\rangle\left|j_{2} m_{2}\right\rangle & =\sum_{j}\left(j_{1} m_{1} j_{2} m_{2} \mid j m\right)|j m\rangle, \\
|j m\rangle & =\sum_{m_{1} m_{2}}\left(j_{1} m_{1} j_{2} m_{2} \mid j m\right)\left|j_{1} m_{1}\right\rangle\left|j_{2} m_{2}\right\rangle,
\end{aligned}
$$

$$
\text { with } m_{1}+m_{2}=m \text {. }
$$

A table of Clebsch-Gordan coefficients can be found in reference [7]. 


$$
\begin{aligned}
& D_{m s}^{j}(\alpha, \beta, \gamma) \equiv\langle j m|R(\alpha, \beta, \gamma)| j s\rangle \\
& D_{m s}^{j}(\alpha, \beta, \gamma)=e^{-i m \alpha} d_{m s}^{j}(\beta) e^{-i s \gamma} \\
& \int_{S^{3}} \mathrm{~d} \Omega D_{m s}^{j *}(\alpha, \beta, \gamma) D_{m^{\prime} s^{\prime}}^{j^{\prime}}(\alpha, \beta, \gamma)=\frac{8 \pi^{2}}{2 j+1} \delta_{j j^{\prime}} \delta_{m m^{\prime}} \delta_{s s^{\prime}} \\
& \int_{S^{2}} \mathrm{~d} \Omega D_{m s}^{j *}(\alpha, \beta, 0) D_{m^{\prime} s}^{j^{\prime}}(\alpha, \beta, 0)=\frac{4 \pi}{2 j+1} \delta_{j j^{\prime}} \delta_{m m^{\prime}} \\
& \int_{0}^{\pi} \mathrm{d} \beta \sin \beta d_{m s}^{j}(\beta) d_{m s}^{j^{\prime}}(\beta)=\frac{2}{2 j+1} \delta_{j j^{\prime}} \\
& D_{m_{1} s_{1}}^{j_{1}}(\alpha, \beta, \gamma) D_{m_{2} s_{2}}^{j_{2}}(\alpha, \beta, \gamma)= \\
& \sum_{j_{3}}\left(j_{1} m_{1} j_{2} m_{2} \mid j_{3} m_{1}+m_{2}\right)\left(j_{1} s_{1} j_{2} s_{2} \mid j_{3} s_{1}+s_{2}\right) D_{m_{1}+m_{2} s_{1}+s_{2}}^{j_{3}}(\alpha, \beta, \gamma) \\
& D_{m_{1} s_{1}}^{j_{1}}(\alpha, \beta, \gamma) D_{m_{3} s_{3}}^{j_{3 *}}(\alpha, \beta, \gamma)= \\
& \sum_{j_{2}}\left(\frac{2 j_{2}+1}{2 j_{3}+1}\right)\left(j_{1} m_{1} j_{2} m_{1}-m_{3} \mid j_{3} m_{3}\right)\left(j_{1} s_{1} j_{2} s_{1}-s_{3} \mid j_{3} s_{3}\right) D_{m_{1}-m_{3} s_{1}-s_{3}}^{j_{2} *}(\alpha, \beta, \gamma) \\
& D_{m 0}^{l}(\alpha, \beta, 0)=\sqrt{\frac{4 \pi}{2 l+1}} Y_{m}^{l *}(\beta, \alpha) \\
& d_{m m^{\prime}}^{j}(\pi)=(-1)^{j-m} \delta_{m-m^{\prime}}
\end{aligned}
$$




\section{Beam-Helicity Asymmetry Data Tables}

\begin{tabular}{l|l|l}
\hline$\phi(\mathrm{rad})$ & $I^{\odot}$ & $\sigma_{\text {stat }}\left(I^{\odot}\right)$ \\
\hline-2.905 & 0.0399141 & 0.00549746 \\
-2.53302 & 0.128643 & 0.00328471 \\
-2.15023 & 0.0628638 & 0.00269081 \\
-1.76423 & -0.00914769 & 0.00243327 \\
-1.38458 & -0.0955621 & 0.0025775 \\
-1.01194 & -0.129561 & 0.00351956 \\
-0.632692 & -0.0703597 & 0.00634946 \\
-0.197412 & 0.00659814 & 0.00946058 \\
0.195121 & 0.000547486 & 0.00965746 \\
0.633444 & 0.0251967 & 0.00633783 \\
1.01227 & 0.124833 & 0.00351233 \\
1.38455 & 0.0793987 & 0.00256984 \\
1.76414 & -0.00123999 & 0.00241866 \\
2.15006 & -0.0906038 & 0.00267398 \\
2.53318 & -0.130086 & 0.00328837 \\
2.90405 & -0.0792164 & 0.00553264
\end{tabular}

Table A1: Pion Data Points for Fig. 4.4.

\begin{tabular}{l|l|l}
\hline$\phi(\mathrm{rad})$ & $I^{\odot}$ & $\sigma_{\text {stat }}\left(I^{\odot}\right)$ \\
\hline-2.93746 & -0.0118352 & 0.0151409 \\
-2.5179 & 0.0140214 & 0.00887212 \\
-2.155 & -0.169374 & 0.00684003 \\
-1.77571 & -0.26273 & 0.00701817 \\
-1.38766 & -0.253313 & 0.00831093 \\
-1.00229 & -0.144872 & 0.0106896 \\
-0.623236 & -0.0469769 & 0.0164551 \\
-0.19884 & 0.018303 & 0.0226071 \\
0.198136 & -0.0390006 & 0.0232467 \\
0.623999 & 0.0592048 & 0.0172081 \\
1.00209 & 0.158464 & 0.0109512 \\
1.38947 & 0.253 & 0.00847152 \\
1.774 & 0.241614 & 0.00717302 \\
2.15523 & 0.184004 & 0.00697577 \\
2.51961 & -0.00780698 & 0.00886164 \\
2.93583 & -0.0465425 & 0.0149465
\end{tabular}

Table A2: Kaon Data Points for Fig. 4.4.

\begin{tabular}{l|l|l|l|l|l|l}
\hline$W(\mathrm{GeV})$ & $c_{1}$ & $\sigma_{\text {stat }}\left(c_{1}\right)$ & $c_{2}$ & $\sigma_{\text {stat }}\left(c_{2}\right)$ & $c_{3}$ & $\sigma_{\text {stat }}\left(c_{3}\right)$ \\
\hline 1.7805 & -0.053003 & 0.0366963 & 0.00830303 & 0.0370443 & -0.0320014 & 0.0370228 \\
1.82321 & -0.00172469 & 0.0109846 & 0.0267856 & 0.0110888 & -0.0310717 & 0.0111137 \\
\hline
\end{tabular}


Continued from previous page

\begin{tabular}{|c|c|c|c|c|c|c|}
\hline 1.87398 & 0.0449405 & 0.0253239 & 0.0542271 & 0.0255514 & -0.010869 & 0.0256339 \\
\hline 1.9198 & 0.0448022 & 0.0169042 & 0.0706845 & 0.0170297 & -0.0264768 & 0.0170885 \\
\hline 1.96882 & 0.0446212 & 0.0127732 & 0.127895 & 0.012861 & -0.0284799 & 0.012921 \\
\hline 2.01652 & 0.0522394 & 0.0224078 & 0.133546 & 0.0225719 & -0.0623813 & 0.0226923 \\
\hline 2.06453 & 0.0382675 & 0.00497087 & 0.179577 & 0.00573854 & -0.0417519 & 0.00592728 \\
\hline 2.11405 & 0.0322943 & 0.00524324 & 0.176191 & 0.00605304 & -0.0483717 & 0.00629119 \\
\hline 2.16102 & 0.0336572 & 0.00529454 & 0.146512 & 0.00611457 & -0.0538234 & 0.00636285 \\
\hline 2.20914 & 0.0233448 & 0.00574616 & 0.151922 & 0.0066282 & -0.0367166 & 0.00690874 \\
\hline 2.25657 & 0.011507 & 0.00593462 & 0.11115 & 0.00684211 & -0.0577347 & 0.00712524 \\
\hline 2.30609 & 0.0021907 & 0.00625522 & 0.112953 & 0.00720955 & -0.0573683 & 0.00750009 \\
\hline 2.35527 & -0.00272769 & 0.006617 & 0.082577 & 0.00761469 & -0.0616457 & 0.00791919 \\
\hline 2.40354 & -0.0165154 & 0.00776896 & 0.0839484 & 0.0089529 & -0.0845576 & 0.009231 \\
\hline 2.45241 & -0.0278888 & 0.0073879 & 0.0864257 & 0.00848382 & -0.0532507 & 0.00875764 \\
\hline 2.50044 & -0.0149099 & 0.00734358 & 0.0810425 & 0.00843828 & -0.0374129 & 0.00864856 \\
\hline 2.54104 & -0.0397746 & 0.00912905 & 0.0889266 & 0.0104693 & -0.0618859 & 0.0107107 \\
\hline 2.6069 & -0.0345995 & 0.013678 & 0.0907428 & 0.015731 & -0.0811243 & 0.016022 \\
\hline 2.64604 & -0.0343632 & 0.0101079 & 0.0515605 & 0.0115729 & -0.0534663 & 0.0117207 \\
\hline 2.69475 & -0.0145134 & 0.0107049 & 0.0858956 & 0.0122626 & -0.0495159 & 0.012365 \\
\hline 2.74394 & -0.00713492 & 0.010485 & 0.0734345 & 0.0119958 & -0.0448202 & 0.0120332 \\
\hline 2.79001 & 0.0303981 & 0.00958747 & 0.0975896 & 0.0109588 & -0.0347238 & 0.0110006 \\
\hline 2.83915 & 0.0235629 & 0.0106982 & 0.0923843 & 0.0122126 & -0.0337375 & 0.0122018 \\
\hline 2.88654 & 0.0234419 & 0.011103 & 0.0353271 & 0.0126523 & -0.0472993 & 0.0127509 \\
\hline 2.93601 & 0.0369615 & 0.0121123 & 0.0828468 & 0.0138424 & -0.0250327 & 0.0137468 \\
\hline 2.9843 & 0.0389724 & 0.0136316 & 0.0276759 & 0.0155243 & -0.0588736 & 0.0154153 \\
\hline 3.03583 & 0.0192387 & 0.0158643 & 0.0379267 & 0.018064 & 0.0128096 & 0.0180433 \\
\hline 3.08067 & 0.057979 & 0.0152839 & 0.0209742 & 0.0174312 & -0.0691353 & 0.0173912 \\
\hline 3.12984 & 0.0704504 & 0.0176237 & 0.0299129 & 0.0200234 & -0.0656594 & 0.0200416 \\
\hline 3.17773 & 0.077053 & 0.0194075 & -0.0148145 & 0.0220555 & -0.0584359 & 0.0217725 \\
\hline 3.2271 & 0.122339 & 0.0209053 & 0.0349276 & 0.0237418 & -0.038877 & 0.0233688 \\
\hline 3.2749 & 0.057025 & 0.0241202 & -0.0571087 & 0.0272635 & -0.0391977 & 0.0273305 \\
\hline
\end{tabular}

Table A3: Data Points for Fig. 4.5a.

\begin{tabular}{l|l|l|l|l|l|l}
\hline$W(\mathrm{GeV})$ & $c_{1}$ & $\sigma_{\text {stat }}\left(c_{1}\right)$ & $c_{2}$ & $\sigma_{\text {stat }}\left(c_{2}\right)$ & $c_{3}$ & $\sigma_{\text {stat }}\left(c_{3}\right)$ \\
\hline 2.1335 & 0.304683 & 0.0417645 & 0.173721 & 0.0477987 & 0.0350634 & 0.0441816 \\
2.19142 & 0.173421 & 0.0337312 & 0.0529572 & 0.0383793 & -0.135053 & 0.0371558 \\
2.25031 & 0.131643 & 0.0276692 & 0.0728267 & 0.0313312 & -0.0398813 & 0.0315137 \\
2.31162 & 0.150341 & 0.0229804 & 0.0694745 & 0.025877 & -0.0943244 & 0.0266029 \\
2.37024 & 0.133858 & 0.0197214 & 0.077208 & 0.0221815 & -0.0544982 & 0.0229384 \\
2.43254 & 0.108163 & 0.0172455 & 0.109749 & 0.019523 & -0.0980525 & 0.0200075 \\
2.49085 & 0.122129 & 0.0140691 & 0.0428448 & 0.0158984 & -0.0967163 & 0.0165745 \\
2.53882 & 0.131952 & 0.0165569 & 0.0720078 & 0.0187667 & -0.118512 & 0.0195411 \\
2.61844 & 0.170484 & 0.017356 & 0.0282977 & 0.0196374 & -0.119788 & 0.0204168 \\
2.6694 & 0.204479 & 0.0142474 & 0.0461814 & 0.0160806 & -0.13633 & 0.0168348 \\
2.72929 & 0.211162 & 0.01326 & 0.0259386 & 0.0149556 & -0.0707303 & 0.015477 \\
2.78868 & 0.189461 & 0.0106932 & -0.0237354 & 0.012006 & -0.0978814 & 0.0123404 \\
2.85006 & 0.212568 & 0.0107591 & -0.0229689 & 0.012031 & -0.0791294 & 0.0123729 \\
2.90906 & 0.201487 & 0.0108994 & -0.0230122 & 0.012165 & -0.0654844 & 0.0124343 \\
2.96893 & 0.219153 & 0.0114012 & -0.0696873 & 0.0127245 & -0.0859753 & 0.0130402 \\
3.03089 & 0.226797 & 0.0125707 & -0.12836 & 0.0140031 & -0.112136 & 0.0142188 \\
3.08872 & 0.239911 & 0.0122535 & -0.110226 & 0.0136709 & -0.0998197 & 0.0139266 \\
3.14968 & 0.304466 & 0.0135042 & -0.0737566 & 0.0150554 & -0.0841553 & 0.0152874 \\
\hline
\end{tabular}

Continued on next page 
Continued from previous page

\begin{tabular}{l|l|l|l|l|l|l}
3.20945 & 0.282349 & 0.0145097 & -0.127427 & 0.0162028 & -0.105117 & 0.0164644 \\
3.26755 & 0.293117 & 0.0161955 & -0.0944868 & 0.0181414 & -0.0552052 & 0.0183787
\end{tabular}

Table A4: Data Points for Fig. 4.5b.

\begin{tabular}{l|l|l|l|l|l|l}
\hline$M\left(K^{+} K^{-}\right)(\mathrm{GeV})$ & $c_{1}$ & $\sigma_{\text {stat }}\left(c_{1}\right)$ & $c_{2}$ & $\sigma_{\text {stat }}\left(c_{2}\right)$ & $c_{3}$ & $\sigma_{\text {stat }}\left(c_{3}\right)$ \\
\hline 0.993495 & -0.00313223 & 0.0407732 & -0.0953019 & 0.042363 & -0.135838 & 0.0386297 \\
1.02389 & 0.236022 & 0.0164587 & -0.0678719 & 0.018314 & -0.0376849 & 0.0168312 \\
1.08009 & 0.397844 & 0.0201434 & 0.130166 & 0.0229722 & -0.00706929 & 0.0225908 \\
1.13285 & 0.335383 & 0.0189769 & 0.119612 & 0.0217495 & -0.0696385 & 0.0212021 \\
1.18473 & 0.27919 & 0.0175098 & 0.11675 & 0.0201104 & -0.0238489 & 0.0195562 \\
1.237 & 0.226257 & 0.0159181 & 0.0030644 & 0.0183126 & -0.116975 & 0.0177743 \\
1.28886 & 0.241177 & 0.01352 & -0.0264492 & 0.0154795 & -0.0792888 & 0.015173 \\
1.33952 & 0.224895 & 0.0127013 & 0.00272025 & 0.0145225 & -0.0801001 & 0.0146045 \\
1.3918 & 0.220481 & 0.0123858 & -0.0170178 & 0.0140549 & -0.0790517 & 0.0146204 \\
1.44389 & 0.207021 & 0.0123851 & 0.00807184 & 0.0139303 & -0.0641417 & 0.0146535 \\
1.49626 & 0.124274 & 0.0119465 & -0.0201139 & 0.0133375 & -0.12074 & 0.0141025 \\
1.54755 & 0.138145 & 0.0119703 & 0.00920219 & 0.0132544 & -0.107444 & 0.0140615 \\
1.59972 & 0.152682 & 0.0124689 & -0.0325488 & 0.0136736 & -0.0911127 & 0.0145112 \\
1.65165 & 0.133114 & 0.0129883 & -0.0401993 & 0.0141839 & -0.0664507 & 0.0151873 \\
1.70313 & 0.108172 & 0.013781 & -0.0630891 & 0.0148712 & -0.0477777 & 0.0161601 \\
1.75493 & 0.0993941 & 0.0153435 & -0.0290659 & 0.0165058 & -0.0141447 & 0.0178283 \\
1.80675 & 0.0606142 & 0.0180789 & 0.0829734 & 0.0191592 & -0.0442613 & 0.0209726 \\
1.85857 & -0.0205097 & 0.0210402 & 0.0878503 & 0.0221899 & -0.1143 & 0.024153 \\
1.91073 & -0.0132811 & 0.0241731 & 0.0722949 & 0.0253412 & -0.107585 & 0.0273742 \\
1.96237 & 0.0331891 & 0.0280715 & 0.074893 & 0.0295484 & -0.178094 & 0.0322108 \\
2.01464 & 0.0809452 & 0.0323634 & 0.0137441 & 0.0343546 & -0.262702 & 0.0369016 \\
2.06574 & 0.111449 & 0.0404713 & 0.263467 & 0.0431942 & -0.147425 & 0.0447615 \\
2.11911 & 0.213415 & 0.0502053 & 0.0443096 & 0.0515617 & -0.308454 & 0.0557667 \\
2.1693 & -0.0301787 & 0.0672098 & 0.0467826 & 0.0705045 & -0.21276 & 0.074807 \\
2.22003 & 0.128162 & 0.094412 & -0.114164 & 0.0989451 & -0.367228 & 0.10833
\end{tabular}

Table A5: Data Points for Fig. 4.6a.

\begin{tabular}{l|l|l|l|l|l|l}
\hline$M\left(p K^{-}\right)(\mathrm{GeV})$ & $c_{1}$ & $\sigma_{\text {stat }}\left(c_{1}\right)$ & $c_{2}$ & $\sigma_{\text {stat }}\left(c_{2}\right)$ & $c_{3}$ & $\sigma_{\text {stat }}\left(c_{3}\right)$ \\
\hline 1.44051 & 0.103514 & 0.0721041 & 0.0368708 & 0.0756546 & -0.143954 & 0.0745331 \\
1.47564 & 0.00768151 & 0.0262979 & 0.114968 & 0.0292099 & 0.0370268 & 0.0309166 \\
1.51966 & 0.0852753 & 0.0102767 & 0.133096 & 0.0115147 & -0.0796605 & 0.0122502 \\
1.5676 & 0.102576 & 0.0149968 & 0.0158495 & 0.0166836 & -0.132067 & 0.018181 \\
1.61645 & 0.0784109 & 0.0145573 & 0.0287417 & 0.0159242 & -0.116506 & 0.0174546 \\
1.66503 & 0.11836 & 0.0128356 & 0.0293172 & 0.0140617 & -0.0905326 & 0.0153581 \\
1.71177 & 0.181917 & 0.0120891 & 0.108429 & 0.0134007 & -0.0745159 & 0.014182 \\
1.76064 & 0.108211 & 0.0115797 & 0.0672736 & 0.0130262 & -0.0440805 & 0.0133381 \\
1.80835 & 0.113203 & 0.0103138 & 0.0629987 & 0.0116788 & -0.0994217 & 0.011746 \\
1.85434 & 0.147995 & 0.0118864 & -0.0444449 & 0.0134501 & -0.167167 & 0.013519 \\
1.90285 & 0.266376 & 0.0135919 & -0.0476223 & 0.0152951 & -0.130833 & 0.0154751 \\
1.95166 & 0.311682 & 0.0150958 & -0.14534 & 0.0170443 & -0.14165 & 0.0173191 \\
1.99988 & 0.324506 & 0.0155068 & -0.148786 & 0.0176099 & -0.102589 & 0.0176479 \\
\hline
\end{tabular}


Continued from previous page

\begin{tabular}{l|l|l|l|l|l|l}
\hline 2.04758 & 0.343979 & 0.0161923 & -0.143057 & 0.0184017 & -0.127915 & 0.018332 \\
2.09526 & 0.372881 & 0.0171716 & -0.105077 & 0.0194669 & -0.0527759 & 0.0192764 \\
2.14305 & 0.367046 & 0.0191592 & -0.158988 & 0.0218266 & -0.0215855 & 0.0208604 \\
2.19132 & 0.454404 & 0.0213479 & -0.141583 & 0.0241702 & -0.0370387 & 0.0234149 \\
2.23918 & 0.428682 & 0.0235815 & -0.0986076 & 0.0268787 & -0.0451404 & 0.0255344 \\
2.28708 & 0.492244 & 0.0276057 & -0.128699 & 0.0314574 & -0.079326 & 0.0294607 \\
2.33505 & 0.499102 & 0.031448 & -0.139651 & 0.0355831 & -0.0782798 & 0.0331397 \\
2.38346 & 0.405522 & 0.0358143 & -0.346067 & 0.0402409 & -0.155003 & 0.0369607 \\
2.43108 & 0.602077 & 0.041699 & -0.110465 & 0.047302 & 0.0588995 & 0.0448141 \\
2.47792 & 0.521262 & 0.0540676 & -0.188934 & 0.0616334 & -0.0887515 & 0.0552792 \\
2.52622 & 0.59246 & 0.0586979 & -0.058735 & 0.0647263 & 0.0857722 & 0.062696 \\
2.5744 & 0.49133 & 0.0853182 & 0.0352232 & 0.0977562 & 0.0349774 & 0.0915506
\end{tabular}

Table A6: Data Points for Fig. 4.6b.

\begin{tabular}{l|l|l|l|l|l|l}
\hline$M\left(p K^{+}\right)(\mathrm{GeV})$ & $c_{1}$ & $\sigma_{\text {stat }}\left(c_{1}\right)$ & $c_{2}$ & $\sigma_{\text {stat }}\left(c_{2}\right)$ & $c_{3}$ & $\sigma_{\text {stat }}\left(c_{3}\right)$ \\
\hline 1.44068 & 0.0529904 & 0.0877234 & -0.187684 & 0.0938252 & -0.0299811 & 0.0922394 \\
1.47582 & 0.185083 & 0.0283502 & 0.0786347 & 0.0314127 & 0.0333106 & 0.0318412 \\
1.52174 & 0.234197 & 0.019363 & 0.117862 & 0.021767 & -0.0473678 & 0.021737 \\
1.56894 & 0.128629 & 0.016238 & 0.00585167 & 0.0183141 & -0.126623 & 0.0181462 \\
1.61632 & 0.160769 & 0.0147948 & -0.0372823 & 0.0166454 & -0.10741 & 0.016918 \\
1.66422 & 0.162873 & 0.013849 & 0.0488221 & 0.0155996 & -0.0630241 & 0.0160354 \\
1.71225 & 0.17355 & 0.0128778 & 0.0213203 & 0.0144729 & -0.123523 & 0.0149727 \\
1.75984 & 0.166936 & 0.0124145 & -0.00719592 & 0.0139328 & -0.135697 & 0.0144825 \\
1.80771 & 0.210439 & 0.0123106 & 0.0178589 & 0.0138192 & -0.129971 & 0.0143763 \\
1.85575 & 0.211257 & 0.0122969 & 0.0175361 & 0.01381 & -0.0900179 & 0.0143352 \\
1.90359 & 0.201474 & 0.0126345 & 0.00708297 & 0.0141376 & -0.114926 & 0.014704 \\
1.95178 & 0.225737 & 0.0129452 & 0.00205714 & 0.0145273 & -0.0820595 & 0.0149223 \\
1.99937 & 0.21367 & 0.0132931 & -0.0458206 & 0.0149045 & -0.0809346 & 0.0153075 \\
2.04739 & 0.204155 & 0.0135853 & -0.0606264 & 0.0152406 & -0.0545555 & 0.0156697 \\
2.09557 & 0.216917 & 0.0143554 & -0.0535616 & 0.0161341 & -0.0895496 & 0.0164481 \\
2.14349 & 0.179029 & 0.0151138 & -0.0545572 & 0.0170599 & -0.0551485 & 0.0173766 \\
2.19133 & 0.194913 & 0.0162759 & -0.0294233 & 0.0183269 & -0.074146 & 0.0187556 \\
2.23921 & 0.176521 & 0.0179999 & -0.0280808 & 0.020206 & -0.0730928 & 0.0205842 \\
2.28704 & 0.194661 & 0.0197134 & -0.0738765 & 0.022169 & -0.0580349 & 0.0227128 \\
2.33504 & 0.246585 & 0.0229277 & -0.0764071 & 0.0257992 & -0.00274081 & 0.0261224 \\
2.383 & 0.228719 & 0.0261246 & -0.00322405 & 0.0293833 & -0.0228369 & 0.0294155 \\
2.43103 & 0.30649 & 0.0306612 & -0.0212093 & 0.0347289 & 0.0110734 & 0.0344281 \\
2.47823 & 0.268392 & 0.0371335 & -0.0410901 & 0.0417326 & -0.0578377 & 0.0432012 \\
2.52551 & 0.352224 & 0.0450495 & -0.0150458 & 0.0512864 & -0.0469609 & 0.0511798 \\
2.57447 & 0.410503 & 0.0625825 & -0.0814262 & 0.0706031 & 0.114156 & 0.0692821 \\
& & & & &
\end{tabular}

Table A7: Data Points for Fig. 4.6c.

\begin{tabular}{l|l|l|l|l|l|l}
\hline$-t_{\gamma \rightarrow K^{+} K^{-}(\mathrm{GeV})^{2}}$ & $c_{1}$ & $\sigma_{\text {stat }}\left(c_{1}\right)$ & $c_{2}$ & $\sigma_{\text {stat }}\left(c_{2}\right)$ & $c_{3}$ & $\sigma_{\text {stat }}\left(c_{3}\right)$ \\
\hline 0.208489 & 0.146762 & 0.0132051 & -0.0154266 & 0.0142824 & 0.00688461 & 0.0150011 \\
0.341423 & 0.131743 & 0.00950452 & -0.00839036 & 0.010609 & -0.0139708 & 0.0110076 \\
0.497906 & 0.109592 & 0.0101982 & 0.0624243 & 0.0115691 & -0.0301166 & 0.0118134 \\
\hline \multicolumn{4}{|r|}{ Continued on next page }
\end{tabular}


Continued from previous page

\begin{tabular}{l|l|l|l|l|l|l}
\hline 0.658106 & 0.135711 & 0.0111495 & 0.09607 & 0.0127421 & -0.102892 & 0.0129456 \\
0.817854 & 0.213849 & 0.0118212 & 0.105252 & 0.0135593 & -0.149668 & 0.0136682 \\
0.978718 & 0.238819 & 0.0119763 & 0.0748508 & 0.0136495 & -0.196259 & 0.013905 \\
1.13867 & 0.245014 & 0.0126163 & 0.00302493 & 0.0143023 & -0.195412 & 0.0144975 \\
1.29693 & 0.298336 & 0.0133111 & 0.0247016 & 0.014949 & -0.201337 & 0.0151913 \\
1.45784 & 0.300963 & 0.0143197 & -0.0641307 & 0.0159169 & -0.159875 & 0.0161371 \\
1.61696 & 0.294682 & 0.0159252 & -0.099656 & 0.0175795 & -0.131378 & 0.0176779 \\
1.77688 & 0.330456 & 0.0172712 & -0.131039 & 0.0188801 & -0.069862 & 0.0191729 \\
1.93643 & 0.317116 & 0.0190354 & -0.138166 & 0.0207081 & -0.0242717 & 0.0212398 \\
2.09656 & 0.323671 & 0.0206974 & -0.128598 & 0.0223285 & 0.0185012 & 0.0235858 \\
2.25638 & 0.198068 & 0.0237121 & -0.234687 & 0.0254508 & 0.0166261 & 0.0268594 \\
2.41649 & 0.203606 & 0.0261364 & -0.239732 & 0.0276835 & -0.00168794 & 0.0296736 \\
2.57588 & 0.156684 & 0.0290534 & -0.218096 & 0.0313827 & 0.0808142 & 0.0336254 \\
2.73683 & 0.0617276 & 0.0326089 & -0.144353 & 0.0352948 & -0.039982 & 0.0377543 \\
2.89718 & 0.0191718 & 0.0360364 & -0.218884 & 0.0389267 & -0.00769374 & 0.0422569 \\
3.05874 & -0.038551 & 0.041331 & -0.160724 & 0.0449711 & -0.0924482 & 0.0486363 \\
3.21517 & -0.0822544 & 0.0461218 & -0.209314 & 0.0502798 & -0.0178489 & 0.0555284 \\
3.37928 & -0.114257 & 0.051 & -0.103485 & 0.0563176 & -0.221239 & 0.0590235 \\
3.53525 & -0.107053 & 0.0566033 & -0.0931968 & 0.0620525 & -0.151485 & 0.0679019 \\
3.69913 & -0.0885922 & 0.0622739 & 0.139312 & 0.0691881 & -0.0594696 & 0.0745721 \\
3.85547 & -0.0606241 & 0.0672771 & -0.221668 & 0.0725611 & -0.0359962 & 0.0802449 \\
4.0214 & -0.16526 & 0.0761286 & -0.295557 & 0.0850294 & -0.110422 & 0.0901838
\end{tabular}

Table A8: Data Points for Fig. 4.7a.

\begin{tabular}{l|l|l|l|l|l|l}
\hline$-t_{\gamma \rightarrow K^{+}}(\mathrm{GeV})^{2}$ & $c_{1}$ & $\sigma_{\text {stat }}\left(c_{1}\right)$ & $c_{2}$ & $\sigma_{\text {stat }}\left(c_{2}\right)$ & $c_{3}$ & $\sigma_{\text {stat }}\left(c_{3}\right)$ \\
\hline 0.488619 & 0.138796 & 0.0208963 & 0.114267 & 0.0234184 & -0.027849 & 0.0159592 \\
0.643362 & 0.158597 & 0.0138556 & -0.00890431 & 0.0158176 & -0.00486254 & 0.0127638 \\
0.79951 & 0.230669 & 0.0112723 & -0.0299224 & 0.0127175 & -0.0486087 & 0.0118823 \\
0.957809 & 0.274512 & 0.0108741 & -0.0490856 & 0.0121852 & -0.0595733 & 0.0123823 \\
1.11584 & 0.332984 & 0.0114264 & -0.041876 & 0.0127485 & -0.0685579 & 0.0137268 \\
1.27516 & 0.338181 & 0.0129262 & -0.0453426 & 0.0144127 & -0.0958505 & 0.0158853 \\
1.43473 & 0.292652 & 0.0149385 & -0.0671557 & 0.0167101 & -0.0934682 & 0.0184081 \\
1.59493 & 0.24233 & 0.0177588 & 0.00727888 & 0.0199229 & -0.0894014 & 0.021728 \\
1.75548 & 0.140853 & 0.0212846 & -0.0319264 & 0.0238518 & -0.122568 & 0.0256689 \\
1.91938 & 0.118604 & 0.0243327 & -0.00283479 & 0.027419 & 0.0122465 & 0.028281 \\
2.07787 & -0.000491832 & 0.0274203 & 0.0301768 & 0.0307576 & -0.0302618 & 0.031315 \\
2.23635 & 0.0252694 & 0.0308322 & 0.0446396 & 0.0345904 & -0.00689238 & 0.0344888 \\
2.40068 & 0.0732663 & 0.0350897 & 0.0814829 & 0.0393009 & 0.00682288 & 0.037862 \\
2.55707 & 0.101408 & 0.0375723 & 0.01453 & 0.0418241 & -0.0413891 & 0.0398447 \\
2.71719 & 0.0882978 & 0.0415754 & 0.0154432 & 0.0460492 & 0.0448518 & 0.043795 \\
2.87829 & -0.0192501 & 0.0490822 & 0.0681121 & 0.0544937 & 0.018164 & 0.0492655 \\
3.03662 & 0.256462 & 0.0510954 & -0.0743478 & 0.0562191 & -0.0546445 & 0.0519874 \\
3.1979 & 0.0925215 & 0.0547268 & -0.0371469 & 0.0595547 & 0.0240124 & 0.0561424 \\
3.35541 & 0.0025618 & 0.0620833 & 0.173156 & 0.0680309 & -0.0674297 & 0.0608911 \\
3.51897 & -0.0239993 & 0.0669333 & 0.0901597 & 0.0740769 & -0.0428655 & 0.068118 \\
3.67862 & 0.123441 & 0.0692082 & -0.0555199 & 0.0757902 & 0.15117 & 0.071995 \\
3.83825 & 0.251592 & 0.0715084 & -0.2724 & 0.0755379 & 0.160244 & 0.0716008 \\
3.99831 & 0.144664 & 0.100064 & -0.275805 & 0.11087 & 0.127776 & 0.0981338 \\
4.15837 & -0.15375 & 0.102562 & -0.0501502 & 0.111311 & 0.116402 & 0.0982805 \\
4.31899 & 0.366783 & 0.113346 & -0.406919 & 0.13077 & 0.10607 & 0.123591 \\
& & & & &
\end{tabular}

Table A9: Data Points for Fig. 4.7b. 


\begin{tabular}{l|l|l}
\hline$\phi(\mathrm{rad})$ & $I^{\odot}$ & $\sigma_{\text {stat }}\left(I^{\odot}\right)$ \\
\hline-2.94536 & -0.046866 & 0.0230102 \\
-2.51537 & 0.0168797 & 0.0161016 \\
-2.14222 & 0.0711579 & 0.0105274 \\
-1.75809 & 0.171811 & 0.0087046 \\
-1.37011 & 0.236847 & 0.00787604 \\
-0.983422 & 0.212959 & 0.00754649 \\
-0.604252 & 0.154042 & 0.00854066 \\
-0.21737 & 0.056629 & 0.0107009 \\
0.222059 & -0.0515227 & 0.0101272 \\
0.602788 & -0.146177 & 0.00846349 \\
0.984341 & -0.222828 & 0.00739433 \\
1.36992 & -0.242008 & 0.00771868 \\
1.75813 & -0.191107 & 0.00856316 \\
2.14079 & -0.0546141 & 0.0105836 \\
2.51714 & -0.0381842 & 0.0159583 \\
2.94662 & 0.0026795 & 0.0227606
\end{tabular}

Table A10: Data Points for Fig. 4.8.

\begin{tabular}{l|l|l|l|l|l|l}
\hline$W(\mathrm{GeV})$ & $c_{1}$ & $\sigma_{\text {stat }}\left(c_{1}\right)$ & $c_{2}$ & $\sigma_{\text {stat }}\left(c_{2}\right)$ & $c_{3}$ & $\sigma_{\text {stat }}\left(c_{3}\right)$ \\
\hline 2.13332 & -0.173791 & 0.032778 & -0.0908573 & 0.0366321 & 0.0476796 & 0.0409505 \\
2.19104 & -0.160182 & 0.0273813 & -0.0821406 & 0.0307983 & 0.0277748 & 0.0338361 \\
2.25026 & -0.0833264 & 0.0237883 & -0.058831 & 0.0268218 & 0.0405591 & 0.0287388 \\
2.31138 & -0.121243 & 0.0206031 & -0.082649 & 0.0230875 & 0.0213117 & 0.0247051 \\
2.37034 & -0.0970004 & 0.0183313 & -0.0951546 & 0.020391 & 0.0158424 & 0.021613 \\
2.43256 & -0.0644231 & 0.0160584 & -0.0631851 & 0.0178036 & 0.0211165 & 0.0189662 \\
2.49081 & -0.10117 & 0.0134242 & -0.0691903 & 0.014913 & 0.0587723 & 0.0156268 \\
2.53886 & -0.0871964 & 0.0158649 & -0.125232 & 0.017688 & 0.0353108 & 0.0185476 \\
2.61835 & -0.160953 & 0.0168461 & -0.0757897 & 0.0187311 & 0.0260497 & 0.0194569 \\
2.66926 & -0.193061 & 0.0140803 & -0.0770313 & 0.0156544 & 0.0299512 & 0.0161997 \\
2.72916 & -0.194963 & 0.0132035 & -0.0861508 & 0.0147489 & 0.0473801 & 0.0149623 \\
2.78854 & -0.199538 & 0.0107235 & -0.0543908 & 0.0119073 & 0.0403228 & 0.0120956 \\
2.84995 & -0.216041 & 0.0110109 & -0.0686655 & 0.0123221 & 0.0110639 & 0.0121621 \\
2.90877 & -0.199221 & 0.0113139 & -0.0734648 & 0.0126904 & 0.0295253 & 0.0123245 \\
2.96891 & -0.251056 & 0.0118577 & -0.0541383 & 0.0133432 & 0.0252375 & 0.0129081 \\
3.03076 & -0.271544 & 0.0132352 & -0.0716323 & 0.0149634 & 0.0835096 & 0.01441 \\
3.08848 & -0.288657 & 0.0131862 & -0.0469154 & 0.0148656 & 0.037823 & 0.0140251 \\
3.14962 & -0.335873 & 0.0145997 & -0.0753155 & 0.0164248 & 0.0238627 & 0.0157634 \\
3.20942 & -0.337699 & 0.0158679 & -0.0648856 & 0.0180028 & 0.0591054 & 0.016972 \\
3.26768 & -0.333794 & 0.0178091 & -0.0587308 & 0.0201508 & -0.0178215 & 0.0188259
\end{tabular}

Table A11: Data Points for Fig. 4.9.

\begin{tabular}{l|l|l|l|l|l|l}
\hline$M\left(K^{+} K^{-}\right)(\mathrm{GeV})$ & $c_{1}$ & $\sigma_{\text {stat }}\left(c_{1}\right)$ & $c_{2}$ & $\sigma_{\text {stat }}\left(c_{2}\right)$ & $c_{3}$ & $\sigma_{\text {stat }}\left(c_{3}\right)$ \\
\hline 0.994734 & 0.0714684 & 0.0442488 & 0.122437 & 0.0500809 & 0.180676 & 0.0453874 \\
1.02385 & -0.244577 & 0.0157292 & 0.0703473 & 0.018326 & 0.0307839 & 0.0174838 \\
1.08101 & -0.315025 & 0.0178759 & -0.0275667 & 0.0198905 & 0.0725471 & 0.0227777 \\
\hline
\end{tabular}


Continued from previous page

\begin{tabular}{l|l|l|l|l|l|l}
\hline 1.13306 & -0.240679 & 0.0154493 & -0.115557 & 0.0171509 & 0.126722 & 0.0195028 \\
1.18483 & -0.188187 & 0.0142301 & -0.128938 & 0.015783 & 0.115702 & 0.0175681 \\
1.23707 & -0.209904 & 0.0128988 & -0.142072 & 0.0142434 & 0.0550039 & 0.0155804 \\
1.28847 & -0.23718 & 0.0116755 & -0.111782 & 0.012878 & 0.00660516 & 0.0137283 \\
1.3393 & -0.206366 & 0.0118169 & -0.114973 & 0.0130552 & 0.020197 & 0.0134562 \\
1.3916 & -0.2037 & 0.0127446 & -0.115666 & 0.0141072 & -0.0143501 & 0.0140242 \\
1.44379 & -0.207105 & 0.0133572 & -0.0659429 & 0.0150322 & 0.000989716 & 0.0144013 \\
1.4961 & -0.135086 & 0.0130906 & -0.0664615 & 0.0147371 & 0.0468924 & 0.0140981 \\
1.54736 & -0.122293 & 0.0131621 & -0.0834331 & 0.014985 & 0.0215897 & 0.0142325 \\
1.59959 & -0.136882 & 0.0138177 & -0.0905607 & 0.015787 & 0.0160402 & 0.0148322 \\
1.65145 & -0.114761 & 0.0145294 & -0.10441 & 0.0167384 & 0.0256141 & 0.0154029 \\
1.70297 & -0.0779735 & 0.0158367 & -0.104214 & 0.0182585 & 0.0160928 & 0.0169592 \\
1.75481 & -0.0524973 & 0.017556 & -0.0917542 & 0.0200981 & -0.000590234 & 0.0187903 \\
1.80681 & -0.0124688 & 0.020016 & -0.014765 & 0.0229242 & 0.0185632 & 0.0218883 \\
1.85862 & 0.0833888 & 0.022829 & -0.0268269 & 0.0262083 & 0.0367607 & 0.0256137 \\
1.91056 & 0.0490103 & 0.0258116 & 0.0379574 & 0.0294477 & 0.0688521 & 0.0285115 \\
1.96229 & -0.00511379 & 0.029144 & 0.0905281 & 0.0334571 & -0.0299662 & 0.0336208 \\
2.01495 & -0.0321192 & 0.035005 & -0.00394887 & 0.0405113 & -0.0798108 & 0.0393415 \\
2.06596 & 0.0375398 & 0.0432417 & -0.109345 & 0.0495397 & -0.00162458 & 0.0485457 \\
2.11854 & -0.0650438 & 0.0534164 & -0.128913 & 0.0616928 & -0.0493627 & 0.0594956 \\
2.16899 & 0.124064 & 0.0679718 & -0.131074 & 0.0796449 & -0.140167 & 0.0782123 \\
2.21932 & 0.011451 & 0.092996 & -0.147799 & 0.104704 & 0.179583 & 0.106105
\end{tabular}

Table A12: Data Points for Fig. 4.11a.

\begin{tabular}{l|l|l|l|l|l|l}
\hline$M\left(p K^{-}\right)(\mathrm{GeV})$ & $c_{1}$ & $\sigma_{\text {stat }}\left(c_{1}\right)$ & $c_{2}$ & $\sigma_{\text {stat }}\left(c_{2}\right)$ & $c_{3}$ & $\sigma_{\text {stat }}\left(c_{3}\right)$ \\
\hline 1.4397 & -0.0804 & 0.0651127 & -0.093692 & 0.0652399 & 0.011113 & 0.0645597 \\
1.47562 & 0.0284343 & 0.0250727 & -0.100445 & 0.0276214 & -0.0672262 & 0.0289859 \\
1.51937 & -0.0185535 & 0.00986005 & -0.16989 & 0.0112137 & -0.00438776 & 0.0116317 \\
1.56756 & -0.113058 & 0.0151797 & -0.0436379 & 0.0173729 & 0.0431224 & 0.0175613 \\
1.61658 & -0.071842 & 0.0151513 & -0.0320228 & 0.0172199 & 0.0236773 & 0.0171932 \\
1.66507 & -0.0902278 & 0.0133334 & -0.136857 & 0.0150705 & 0.0236242 & 0.0152318 \\
1.7119 & -0.144527 & 0.0122623 & -0.0443419 & 0.0136893 & 0.0408968 & 0.0138764 \\
1.76091 & -0.0946784 & 0.0113049 & 0.0598325 & 0.0125313 & 0.00609321 & 0.0128336 \\
1.80816 & -0.108901 & 0.00983462 & 0.0418363 & 0.0109697 & 0.0302603 & 0.0112772 \\
1.85428 & -0.164655 & 0.0114001 & -0.0479499 & 0.0126901 & 0.0664385 & 0.0128777 \\
1.90279 & -0.293914 & 0.0134855 & -0.0316544 & 0.0148916 & -0.00931956 & 0.0151754 \\
1.95174 & -0.361431 & 0.0153308 & -0.112986 & 0.0171063 & 0.040979 & 0.0168363 \\
1.99994 & -0.405866 & 0.0157806 & -0.0751328 & 0.0176607 & 0.0695088 & 0.0169006 \\
2.04752 & -0.460841 & 0.0163893 & -0.0140295 & 0.0185372 & 0.144586 & 0.0175277 \\
2.09534 & -0.455991 & 0.0173387 & -0.0285825 & 0.0195086 & 0.126735 & 0.018486 \\
2.14305 & -0.469174 & 0.0191645 & -0.0442944 & 0.0215382 & 0.0811701 & 0.0202091 \\
2.19129 & -0.480686 & 0.0218031 & -0.17243 & 0.0244244 & 0.11217 & 0.0226699 \\
2.23884 & -0.465528 & 0.0258745 & -0.142505 & 0.0292097 & 0.100254 & 0.0267528 \\
2.28679 & -0.537467 & 0.0296279 & -0.215597 & 0.032817 & 0.137388 & 0.0291827 \\
2.33467 & -0.4507 & 0.0360687 & -0.328778 & 0.0402614 & 0.139903 & 0.0351854 \\
2.38351 & -0.575401 & 0.0432309 & -0.130906 & 0.0475175 & 0.0349739 & 0.0410379 \\
2.43062 & -0.598335 & 0.053843 & -0.182899 & 0.0586167 & 0.00639465 & 0.0503029 \\
2.47834 & -0.707414 & 0.0673847 & 0.0208086 & 0.0723948 & -0.101914 & 0.0623611 \\
2.52671 & -0.528256 & 0.084292 & -0.159368 & 0.0913814 & -0.0840025 & 0.0780815 \\
2.57409 & -0.424433 & 0.122673 & -0.3166 & 0.136105 & 0.233606 & 0.111256
\end{tabular}

Table A13: Data Points for Fig. 4.11b. 


\begin{tabular}{l|l|l|l|l|l|l}
\hline$M\left(p K^{+}\right)(\mathrm{GeV})$ & $c_{1}$ & $\sigma_{\text {stat }}\left(c_{1}\right)$ & $c_{2}$ & $\sigma_{\text {stat }}\left(c_{2}\right)$ & $c_{3}$ & $\sigma_{\text {stat }}\left(c_{3}\right)$ \\
\hline 1.44169 & -0.063719 & 0.100817 & -0.182259 & 0.10982 & -0.0902982 & 0.112549 \\
1.47618 & -0.154838 & 0.0285033 & -0.00913932 & 0.0319506 & 0.0194965 & 0.0327118 \\
1.52211 & -0.159516 & 0.0180161 & -0.153336 & 0.0203099 & -0.00606533 & 0.0212847 \\
1.56912 & -0.0995315 & 0.0150321 & -0.134291 & 0.016943 & 0.0761067 & 0.0175715 \\
1.61645 & -0.149367 & 0.0140533 & -0.116625 & 0.0157208 & -0.00190352 & 0.0163469 \\
1.66432 & -0.125229 & 0.0135616 & -0.107966 & 0.0151632 & 0.0292778 & 0.015569 \\
1.71208 & -0.141945 & 0.0130464 & -0.122786 & 0.0145933 & 0.051319 & 0.0146531 \\
1.75988 & -0.161663 & 0.0126659 & -0.0740919 & 0.0142255 & 0.0393444 & 0.0143302 \\
1.80775 & -0.192794 & 0.0126178 & -0.095203 & 0.014171 & 0.0668368 & 0.0141622 \\
1.85567 & -0.196636 & 0.012699 & -0.07817 & 0.014292 & 0.0174345 & 0.0140941 \\
1.90362 & -0.197974 & 0.0130903 & -0.06094 & 0.0147592 & 0.0479585 & 0.0144457 \\
1.95184 & -0.228782 & 0.0132606 & -0.0624854 & 0.01497 & 0.0251212 & 0.0145628 \\
1.99958 & -0.238039 & 0.013484 & -0.0412336 & 0.015245 & 0.0393838 & 0.0147819 \\
2.04761 & -0.243327 & 0.0138284 & -0.00803802 & 0.015594 & 0.0364916 & 0.0151379 \\
2.09563 & -0.246485 & 0.0142147 & -0.0644306 & 0.0159028 & 0.0460143 & 0.0157266 \\
2.14333 & -0.200445 & 0.014992 & -0.0578639 & 0.0167179 & 0.0457293 & 0.0166155 \\
2.19126 & -0.220161 & 0.0161224 & -0.0168388 & 0.0179604 & 0.0504345 & 0.0178002 \\
2.23914 & -0.197287 & 0.0178489 & -0.0278451 & 0.019938 & -0.0202528 & 0.0198739 \\
2.28691 & -0.22257 & 0.0196343 & -0.0554422 & 0.021748 & 0.0266961 & 0.0216883 \\
2.33488 & -0.27948 & 0.0222112 & -0.0144204 & 0.024498 & -0.0298177 & 0.0247762 \\
2.38274 & -0.225485 & 0.0252027 & -0.0478992 & 0.0276621 & -0.0822374 & 0.0282037 \\
2.43098 & -0.309124 & 0.0288902 & -0.0184706 & 0.0317915 & -0.0253158 & 0.0334049 \\
2.4786 & -0.299831 & 0.0355418 & -0.0824041 & 0.039707 & -0.0625214 & 0.0419305 \\
2.52546 & -0.332431 & 0.0442839 & -0.148191 & 0.0488418 & 0.0122495 & 0.0512633 \\
2.57411 & -0.443115 & 0.0579647 & 0.0197497 & 0.0635367 & -0.199555 & 0.0663907
\end{tabular}

Table A14: Data Points for Fig. 4.11c.

\begin{tabular}{l|l|l|l|l|l|l}
\hline$-t_{\gamma \rightarrow K^{+} K^{-}(\mathrm{GeV})^{2}}$ & $c_{1}$ & $\sigma_{\text {stat }}\left(c_{1}\right)$ & $c_{2}$ & $\sigma_{\text {stat }}\left(c_{2}\right)$ & $c_{3}$ & $\sigma_{\text {stat }}\left(c_{3}\right)$ \\
\hline 0.112549 & 1.91356 & 1.03882 & -1.79459 & 0.968318 & 0.422039 & 0.315545 \\
0.366227 & -0.0397572 & 0.0478246 & -0.121949 & 0.0533386 & 0.00340076 & 0.0278737 \\
0.506534 & -0.0673742 & 0.0210729 & -0.00077348 & 0.0242381 & -0.0975207 & 0.0167567 \\
0.660837 & -0.0737254 & 0.0147422 & -0.0900256 & 0.0169297 & -0.0217469 & 0.0147496 \\
0.818018 & -0.138497 & 0.0123445 & -0.145067 & 0.0137116 & -0.00560625 & 0.0144013 \\
0.978145 & -0.187133 & 0.0119759 & -0.168687 & 0.0130924 & -0.0165107 & 0.0149813 \\
1.1385 & -0.205547 & 0.0124781 & -0.20881 & 0.0136536 & 0.0116991 & 0.0159523 \\
1.29686 & -0.235739 & 0.0133009 & -0.267969 & 0.014631 & 0.0801528 & 0.0170829 \\
1.45712 & -0.288711 & 0.0146482 & -0.179342 & 0.0162223 & 0.064444 & 0.0186532 \\
1.61677 & -0.285029 & 0.0167228 & -0.107103 & 0.0186542 & 0.118643 & 0.0208886 \\
1.77694 & -0.354575 & 0.019156 & -0.12654 & 0.0216577 & 0.108414 & 0.0230418 \\
1.93633 & -0.336182 & 0.022435 & -0.0680313 & 0.025635 & 0.146721 & 0.0260017 \\
2.09653 & -0.297017 & 0.0265309 & 0.0336148 & 0.0303296 & 0.157678 & 0.0295322 \\
2.25605 & -0.223828 & 0.0317275 & 0.0546998 & 0.0365446 & 0.0600541 & 0.0337186 \\
2.41542 & -0.13678 & 0.03588 & 0.173388 & 0.0411132 & 0.166524 & 0.0377881 \\
2.57581 & -0.0170028 & 0.0428959 & 0.291487 & 0.0492451 & 0.127397 & 0.0439027 \\
2.73714 & 0.0839596 & 0.0502599 & 0.233927 & 0.0571894 & 0.173378 & 0.0491511 \\
2.89854 & 0.131179 & 0.0519984 & 0.271357 & 0.0591294 & 0.187162 & 0.0530869 \\
3.0635 & 0.252526 & 0.0616124 & 0.346126 & 0.071269 & 0.171436 & 0.0631309 \\
3.2152 & 0.16248 & 0.0692342 & 0.113068 & 0.0789442 & 0.0318883 & 0.0692361 \\
3.38046 & 0.110035 & 0.0728034 & 0.00901776 & 0.0829674 & -0.142905 & 0.07048 \\
3.53682 & 0.176635 & 0.0864407 & 0.0886421 & 0.0996458 & 0.221807 & 0.081942 \\
\hline
\end{tabular}


Continued from previous page

\begin{tabular}{l|l|l|l|l|l|l}
\hline 3.69801 & 0.1247 & 0.0910328 & 0.0252769 & 0.103486 & 0.170052 & 0.0878949 \\
3.85679 & 0.15163 & 0.0975794 & 0.149851 & 0.113367 & -0.0382132 & 0.101338 \\
4.01937 & 0.20076 & 0.1054 & 0.0918695 & 0.117027 & -0.0964455 & 0.10267
\end{tabular}

Table A15: Data Points for Fig. 4.10a.

\begin{tabular}{l|l|l|l|l|l|l}
\hline$-t_{\gamma \rightarrow K^{+}}(\mathrm{GeV})^{2}$ & $c_{1}$ & $\sigma_{\text {stat }}\left(c_{1}\right)$ & $c_{2}$ & $\sigma_{\text {stat }}\left(c_{2}\right)$ & $c_{3}$ & $\sigma_{\text {stat }}\left(c_{3}\right)$ \\
\hline 0.483961 & -0.0210999 & 0.00926596 & 0.0172577 & 0.0103906 & -0.0443142 & 0.0105178 \\
0.640366 & -0.159541 & 0.00856004 & -0.0289412 & 0.0096147 & 0.0343112 & 0.00961337 \\
0.797425 & -0.240781 & 0.00897732 & -0.0966893 & 0.010159 & 0.0683074 & 0.0100267 \\
0.956626 & -0.315758 & 0.0100508 & -0.092908 & 0.0113799 & 0.11532 & 0.0110132 \\
1.11549 & -0.385623 & 0.0114412 & -0.115876 & 0.0128873 & 0.108821 & 0.012493 \\
1.27581 & -0.413399 & 0.0134424 & -0.107907 & 0.015147 & 0.117431 & 0.0146434 \\
1.43546 & -0.341539 & 0.0154896 & -0.140306 & 0.0172912 & 0.0762306 & 0.0168977 \\
1.59591 & -0.270302 & 0.0178936 & -0.173881 & 0.0198263 & 0.0513212 & 0.0197101 \\
1.75627 & -0.161962 & 0.0202434 & -0.121461 & 0.0225758 & -0.000912182 & 0.0226875 \\
1.91878 & -0.0960217 & 0.0222713 & -0.111958 & 0.0245893 & 0.000315787 & 0.0248599 \\
2.07791 & 0.000807737 & 0.0240003 & -0.110739 & 0.0262563 & -0.0060071 & 0.0271208 \\
2.23697 & -0.0298589 & 0.0257869 & -0.0547436 & 0.0279845 & -0.0461827 & 0.0291132 \\
2.39931 & -0.092296 & 0.0275052 & -0.108185 & 0.0301933 & -0.00792 & 0.0323352 \\
2.55853 & -0.100518 & 0.0287882 & -0.0759331 & 0.0314916 & -0.0178409 & 0.0338014 \\
2.71732 & -0.0758975 & 0.0313661 & -0.0990138 & 0.0342702 & -0.0666466 & 0.03757 \\
2.87683 & -0.0159974 & 0.0340745 & -0.133512 & 0.0378944 & -0.0191898 & 0.0412827 \\
3.03678 & -0.206105 & 0.0364204 & -0.0246809 & 0.0400865 & -0.0692592 & 0.0444496 \\
3.19789 & -0.0229865 & 0.0396761 & -0.173585 & 0.0440348 & 0.0867193 & 0.0481135 \\
3.35628 & -0.0964615 & 0.0431695 & -0.210655 & 0.0478391 & -0.0346393 & 0.0538059 \\
3.51907 & -0.0476021 & 0.0467988 & -0.16354 & 0.0521546 & -0.0273303 & 0.0587636 \\
3.67808 & -0.126758 & 0.0526788 & -0.0928521 & 0.0589787 & -0.111451 & 0.0656298 \\
3.83531 & -0.0701525 & 0.0555733 & 0.129644 & 0.0626499 & -0.0643557 & 0.0672652 \\
3.99816 & 0.167074 & 0.0633581 & 0.16851 & 0.071259 & 0.254422 & 0.0779046 \\
4.15897 & 0.126113 & 0.0712661 & -0.00115688 & 0.0810496 & -0.044392 & 0.085528 \\
4.31955 & -0.0497181 & 0.081739 & 0.144789 & 0.0908052 & -0.139001 & 0.10474
\end{tabular}

Table A16: Data Points for Fig. 4.10b.

\begin{tabular}{l|l|l}
\hline$\phi(\mathrm{rad})$ & $I^{\odot}$ & $\sigma_{\text {stat }}\left(I^{\odot}\right)$ \\
\hline-2.91608 & 0.060312 & 0.00868513 \\
-2.54637 & 0.0926191 & 0.00655846 \\
-2.16908 & 0.262277 & 0.00687661 \\
-1.78571 & 0.276479 & 0.00898659 \\
-1.39151 & 0.170198 & 0.0119302 \\
-0.996912 & 0.00934449 & 0.0150932 \\
-0.624245 & -0.0447849 & 0.0213438 \\
-0.208159 & -0.0673789 & 0.0341775 \\
0.206368 & -0.0574193 & 0.0348914 \\
0.626452 & 0.040166 & 0.021455 \\
0.995444 & -0.0257776 & 0.01481 \\
1.39061 & -0.127554 & 0.0117534 \\
\hline \multicolumn{2}{|c}{ Continued on next page }
\end{tabular}


Continued from previous page

\begin{tabular}{l|l|l}
\hline 1.78571 & -0.288414 & 0.00867712 \\
2.16998 & -0.276787 & 0.00673848 \\
2.54639 & -0.0901412 & 0.00656679 \\
2.91534 & -0.0544345 & 0.00836543
\end{tabular}

Table A17: Data Points for Fig. 4.12.

\begin{tabular}{l|l|l|l|l|l|l}
\hline$W(\mathrm{GeV})$ & $c_{1}$ & $\sigma_{\text {stat }}\left(c_{1}\right)$ & $c_{2}$ & $\sigma_{\text {stat }}\left(c_{2}\right)$ & $c_{3}$ & $\sigma_{\text {stat }}\left(c_{3}\right)$ \\
\hline 2.13333 & -0.276297 & 0.0378904 & -0.123437 & 0.0436916 & -0.066823 & 0.0403319 \\
2.19081 & -0.156853 & 0.0327627 & 0.0374236 & 0.0374073 & -0.0356639 & 0.0344389 \\
2.25027 & -0.101219 & 0.0284383 & -0.00438089 & 0.0323145 & -0.0119467 & 0.0297821 \\
2.31131 & -0.0983163 & 0.0243462 & 0.103824 & 0.0273759 & 0.0261894 & 0.0255795 \\
2.37007 & -0.0651771 & 0.0214114 & 0.103282 & 0.0238642 & 0.0477314 & 0.0224293 \\
2.43258 & -0.0964859 & 0.019145 & 0.0133848 & 0.0212637 & 0.079726 & 0.019767 \\
2.49072 & -0.0902232 & 0.016017 & 0.105216 & 0.0179072 & 0.141495 & 0.0164912 \\
2.53882 & -0.081466 & 0.0193208 & 0.122441 & 0.0217125 & 0.120999 & 0.0194619 \\
2.61841 & -0.134481 & 0.0203926 & 0.132438 & 0.02286 & 0.13145 & 0.0206334 \\
2.66936 & -0.209043 & 0.0171302 & 0.104086 & 0.0190411 & 0.172615 & 0.0170136 \\
2.72914 & -0.204056 & 0.0164511 & 0.105876 & 0.0183995 & 0.129417 & 0.0161271 \\
2.78841 & -0.193768 & 0.0135726 & 0.119962 & 0.0150617 & 0.118576 & 0.0130531 \\
2.84987 & -0.176431 & 0.0141783 & 0.16704 & 0.0156315 & 0.0977969 & 0.0133345 \\
2.90898 & -0.179031 & 0.0148422 & 0.170974 & 0.0162097 & 0.1392 & 0.0137041 \\
2.96883 & -0.191817 & 0.0158306 & 0.194987 & 0.0172483 & 0.110747 & 0.0143753 \\
3.03056 & -0.22032 & 0.0177998 & 0.202199 & 0.0194 & 0.113145 & 0.0162263 \\
3.08846 & -0.252732 & 0.0175262 & 0.182239 & 0.0189308 & 0.119388 & 0.0159169 \\
3.14971 & -0.277313 & 0.0195593 & 0.199448 & 0.0211243 & 0.0524716 & 0.0177996 \\
3.20969 & -0.275395 & 0.0217913 & 0.21131 & 0.023454 & 0.0792082 & 0.0193369 \\
3.2675 & -0.249348 & 0.0246619 & 0.17577 & 0.0266999 & 0.00809605 & 0.0218077
\end{tabular}

Table A18: Data Points for Fig. 4.13.

\begin{tabular}{l|l|l|l|l|l|l}
\hline$M\left(K^{+} K^{-}\right)(\mathrm{GeV})$ & $c_{1}$ & $\sigma_{\text {stat }}\left(c_{1}\right)$ & $c_{2}$ & $\sigma_{\text {stat }}\left(c_{2}\right)$ & $c_{3}$ & $\sigma_{\text {stat }}\left(c_{3}\right)$ \\
\hline 0.994698 & 0.0988123 & 0.048472 & 0.190868 & 0.0498269 & 0.176627 & 0.04487 \\
1.02414 & -0.222582 & 0.0177231 & 0.118433 & 0.0193472 & 0.0649063 & 0.0173743 \\
1.08025 & -0.412962 & 0.0212358 & -0.0853199 & 0.0241587 & 0.0550701 & 0.0220039 \\
1.13296 & -0.315517 & 0.020065 & 0.0203945 & 0.0229323 & 0.0826399 & 0.0201841 \\
1.18458 & -0.23069 & 0.0191495 & 0.0510965 & 0.0216607 & 0.0611696 & 0.0186544 \\
1.23709 & -0.173115 & 0.0181591 & 0.181995 & 0.0203315 & 0.102972 & 0.0170767 \\
1.28877 & -0.157937 & 0.0161413 & 0.239113 & 0.017719 & 0.0985236 & 0.0150513 \\
1.33946 & -0.137563 & 0.0156602 & 0.261636 & 0.0169828 & 0.129639 & 0.0146955 \\
1.39171 & -0.145258 & 0.0159531 & 0.216167 & 0.0172476 & 0.0640865 & 0.0151534 \\
1.44383 & -0.174173 & 0.0164807 & 0.166827 & 0.0180817 & 0.105942 & 0.0155599 \\
1.49604 & -0.154385 & 0.016127 & 0.136242 & 0.0177668 & 0.194782 & 0.0151801 \\
1.5473 & -0.126103 & 0.0166015 & 0.131321 & 0.0183271 & 0.133793 & 0.0156072 \\
1.59962 & -0.117062 & 0.0174572 & 0.130843 & 0.0190894 & 0.073387 & 0.0163465 \\
1.65171 & -0.123379 & 0.0180923 & 0.0866523 & 0.0199929 & 0.0592254 & 0.0170189 \\
1.70323 & -0.0639139 & 0.0189906 & 0.0991561 & 0.0212193 & 0.00965761 & 0.0181391 \\
1.75477 & -0.0426654 & 0.0201624 & 0.0762895 & 0.0222364 & -0.0198055 & 0.0196927 \\
\hline
\end{tabular}


Continued from previous page

\begin{tabular}{l|l|l|l|l|l|l}
\hline 1.8067 & -0.0634041 & 0.0227865 & -0.0714118 & 0.0253661 & -0.0134834 & 0.0229536 \\
1.85875 & 0.0417307 & 0.0259253 & -0.0213339 & 0.0291977 & 0.053566 & 0.0263933 \\
1.91055 & 0.0210822 & 0.0296562 & -0.0155579 & 0.0330429 & 0.0989385 & 0.0296441 \\
1.96229 & -0.0744323 & 0.0326447 & -0.157409 & 0.0367271 & 0.052795 & 0.0343051 \\
2.01499 & -0.113951 & 0.0383464 & -0.175802 & 0.0434198 & -0.11007 & 0.0405483 \\
2.06595 & -0.016216 & 0.0461523 & -0.0392022 & 0.0509575 & 0.0657403 & 0.0491103 \\
2.1186 & -0.0725329 & 0.056868 & 0.0914875 & 0.0629945 & 0.0100891 & 0.0594562 \\
2.16947 & -0.00447073 & 0.0698696 & -0.208234 & 0.0785402 & -0.305387 & 0.075262 \\
2.21978 & -0.0367354 & 0.0950245 & 0.00565622 & 0.104171 & 0.0493063 & 0.103226
\end{tabular}

Table A19: Data Points for Fig. 4.15a.

\begin{tabular}{l|l|l|l|l|l|l}
\hline$M\left(p K^{-}\right)(\mathrm{GeV})$ & $c_{1}$ & $\sigma_{\text {stat }}\left(c_{1}\right)$ & $c_{2}$ & $\sigma_{\text {stat }}\left(c_{2}\right)$ & $c_{3}$ & $\sigma_{\text {stat }}\left(c_{3}\right)$ \\
\hline 1.44229 & 0.00135215 & 0.0981267 & 0.0619599 & 0.104785 & 0.263957 & 0.0976638 \\
1.47617 & 0.0664353 & 0.0270645 & 0.043891 & 0.029772 & -0.0321577 & 0.0295162 \\
1.51901 & 0.0123612 & 0.012653 & 0.0757372 & 0.0142139 & -0.072707 & 0.0124384 \\
1.56756 & -0.124786 & 0.0193085 & 0.0379583 & 0.0214897 & 0.0369894 & 0.0189928 \\
1.61639 & -0.0794842 & 0.0192738 & 0.0441728 & 0.0212481 & 0.0580381 & 0.0188497 \\
1.66534 & -0.0493158 & 0.0162941 & 0.141285 & 0.0179688 & 0.040459 & 0.0163563 \\
1.71187 & -0.200116 & 0.015141 & -0.012787 & 0.0166995 & 0.0876011 & 0.0149909 \\
1.76065 & -0.114403 & 0.0144659 & 0.0319205 & 0.0160926 & 0.156013 & 0.0140688 \\
1.80801 & -0.131306 & 0.0130523 & 0.0505381 & 0.0146453 & 0.159978 & 0.0124969 \\
1.85423 & -0.133216 & 0.015515 & 0.180702 & 0.0173794 & 0.171606 & 0.0145582 \\
1.90306 & -0.261215 & 0.0177678 & 0.144743 & 0.0197232 & 0.106374 & 0.0166943 \\
1.95172 & -0.278921 & 0.0196381 & 0.272161 & 0.0219641 & 0.112748 & 0.0185836 \\
1.9998 & -0.326572 & 0.0204292 & 0.239818 & 0.0226834 & 0.149888 & 0.0189779 \\
2.04758 & -0.354805 & 0.0213735 & 0.285984 & 0.0237602 & 0.218487 & 0.0196732 \\
2.09516 & -0.366367 & 0.0222288 & 0.221275 & 0.02447 & 0.134299 & 0.0206425 \\
2.14308 & -0.340489 & 0.0247843 & 0.269621 & 0.0271326 & 0.113269 & 0.0224183 \\
2.1912 & -0.435067 & 0.027087 & 0.272212 & 0.0293766 & 0.12495 & 0.0245967 \\
2.23929 & -0.429265 & 0.0287946 & 0.205413 & 0.0309586 & 0.0773404 & 0.0272022 \\
2.28713 & -0.468096 & 0.0337005 & 0.302642 & 0.0366355 & 0.180515 & 0.0307297 \\
2.33483 & -0.466991 & 0.0387552 & 0.294738 & 0.0419098 & 0.130789 & 0.0349594 \\
2.38365 & -0.376551 & 0.0433397 & 0.393859 & 0.0464522 & 0.0711475 & 0.0397859 \\
2.43112 & -0.522032 & 0.0504538 & 0.247446 & 0.0557277 & 0.0102513 & 0.0483683 \\
2.47833 & -0.486369 & 0.0657607 & 0.262862 & 0.0709172 & -0.0663625 & 0.0600987 \\
2.52638 & -0.610988 & 0.0652215 & 0.0475466 & 0.0697935 & -0.206594 & 0.0653684
\end{tabular}

Table A20: Data Points for Fig. 4.15b.

\begin{tabular}{l|l|l|l|l|l|l}
\hline$M\left(p K^{+}\right)(\mathrm{GeV})$ & $c_{1}$ & $\sigma_{\text {stat }}\left(c_{1}\right)$ & $c_{2}$ & $\sigma_{\text {stat }}\left(c_{2}\right)$ & $c_{3}$ & $\sigma_{\text {stat }}\left(c_{3}\right)$ \\
\hline 1.44033 & -0.0540838 & 0.0834681 & 0.203111 & 0.0890178 & -0.000474474 & 0.0865014 \\
1.47575 & -0.178061 & 0.0281114 & -0.0452807 & 0.0310839 & -0.0186648 & 0.0312125 \\
1.52174 & -0.198614 & 0.0195612 & -0.0164084 & 0.0220408 & -0.0326576 & 0.021166 \\
1.5689 & -0.110884 & 0.0168537 & 0.0760975 & 0.0189464 & 0.0506255 & 0.0178235 \\
1.61617 & -0.128733 & 0.0161185 & 0.111361 & 0.018138 & 0.0428818 & 0.0167905 \\
1.66426 & -0.123345 & 0.0155923 & 0.0887478 & 0.0175679 & 0.0874276 & 0.0161429 \\
1.71214 & -0.122799 & 0.0150574 & 0.154655 & 0.0169353 & 0.122994 & 0.0153872 \\
\hline
\end{tabular}


Continued from previous page

\begin{tabular}{l|l|l|l|l|l|l}
\hline 1.75969 & -0.162667 & 0.0148976 & 0.110402 & 0.0167706 & 0.153182 & 0.015081 \\
1.80759 & -0.1883 & 0.0151175 & 0.139552 & 0.0169886 & 0.158287 & 0.0150876 \\
1.85552 & -0.186932 & 0.0154222 & 0.143188 & 0.0171025 & 0.138401 & 0.0151709 \\
1.90341 & -0.215048 & 0.0165488 & 0.107571 & 0.0182537 & 0.144329 & 0.0156933 \\
1.95162 & -0.203323 & 0.0174434 & 0.173197 & 0.0192368 & 0.143904 & 0.0162395 \\
1.9993 & -0.217214 & 0.01865 & 0.146448 & 0.0204668 & 0.128999 & 0.0168144 \\
2.04728 & -0.195308 & 0.0197423 & 0.169549 & 0.0214449 & 0.121405 & 0.0174204 \\
2.09553 & -0.153992 & 0.0213531 & 0.237763 & 0.0232521 & 0.113501 & 0.0187838 \\
2.14299 & -0.126946 & 0.0230607 & 0.195039 & 0.0247591 & 0.0945772 & 0.0198493 \\
2.19129 & -0.142764 & 0.0252255 & 0.196646 & 0.0266525 & 0.0889826 & 0.0214031 \\
2.23886 & -0.0920265 & 0.0287987 & 0.189301 & 0.0300363 & 0.0378122 & 0.0241679 \\
2.28707 & -0.115906 & 0.0320603 & 0.186728 & 0.0330345 & 0.0222153 & 0.0266887 \\
2.33437 & -0.167606 & 0.0384094 & 0.172737 & 0.0404001 & 0.0037625 & 0.0318803 \\
2.38225 & -0.120364 & 0.0432909 & 0.0678113 & 0.0444222 & -0.143166 & 0.0363204 \\
2.4317 & -0.238725 & 0.05003 & 0.107758 & 0.0517731 & -0.0514778 & 0.042506 \\
2.47818 & -0.0555327 & 0.0614915 & 0.226739 & 0.0643458 & -0.130528 & 0.052804 \\
2.52586 & -0.129344 & 0.0793911 & 0.338346 & 0.0797487 & 0.0543927 & 0.0647783 \\
2.57466 & -0.238882 & 0.100045 & 0.186879 & 0.0920721 & -0.184554 & 0.07957
\end{tabular}

Table A21: Data Points for Fig. 4.15c.

\begin{tabular}{l|l|l|l|l|l|l}
\hline$-t_{\gamma \rightarrow K^{+K^{-}}(\mathrm{GeV})^{2}}$ & $c_{1}$ & $\sigma_{\text {stat }}\left(c_{1}\right)$ & $c_{2}$ & $\sigma_{\text {stat }}\left(c_{2}\right)$ & $c_{3}$ & $\sigma_{\text {stat }}\left(c_{3}\right)$ \\
\hline 0.0706861 & 1.47533 & 0.442231 & -0.0971415 & 0.307762 & -0.793863 & 0.139874 \\
0.2591 & -1.57601 & 0.611086 & -1.43613 & 0.574102 & -0.577912 & 0.192812 \\
0.517035 & -0.018555 & 0.076034 & 0.156779 & 0.0792182 & 0.0958701 & 0.0367017 \\
0.668868 & -0.101568 & 0.034697 & 0.0892141 & 0.0380989 & 0.116738 & 0.0224924 \\
0.822299 & -0.0861943 & 0.0211613 & 0.179996 & 0.023936 & 0.141201 & 0.017687 \\
0.980261 & -0.142073 & 0.0170878 & 0.142715 & 0.0193268 & 0.137031 & 0.0164494 \\
1.1394 & -0.173291 & 0.0159836 & 0.133478 & 0.0179385 & 0.0645784 & 0.0165274 \\
1.29737 & -0.202122 & 0.0155767 & 0.15879 & 0.0174381 & 0.0401101 & 0.0168859 \\
1.45828 & -0.223507 & 0.0155852 & 0.191364 & 0.0174029 & 0.0452474 & 0.0174379 \\
1.61756 & -0.249671 & 0.0162243 & 0.194691 & 0.0180906 & 0.0143883 & 0.0186307 \\
1.77707 & -0.295118 & 0.0170375 & 0.261544 & 0.0190225 & 0.0154706 & 0.0199296 \\
1.93715 & -0.319408 & 0.0181935 & 0.182677 & 0.0203716 & 0.0209688 & 0.0217336 \\
2.09663 & -0.33566 & 0.0194327 & 0.20674 & 0.0216304 & -0.0111566 & 0.0237143 \\
2.25626 & -0.264119 & 0.0220731 & 0.260063 & 0.0245088 & 0.0291873 & 0.0267463 \\
2.41651 & -0.295868 & 0.0246168 & 0.248822 & 0.0270974 & -0.0416488 & 0.0299494 \\
2.57541 & -0.262809 & 0.0283834 & 0.242445 & 0.0310349 & -0.0601902 & 0.0344083 \\
2.73706 & -0.136309 & 0.0339285 & 0.15218 & 0.0371802 & 0.0561719 & 0.03984 \\
2.89625 & -0.125446 & 0.0404779 & 0.160149 & 0.0443129 & 0.0710508 & 0.0454918 \\
3.05846 & -0.00837449 & 0.048108 & 0.0237453 & 0.0520957 & 0.122146 & 0.0536711 \\
3.2162 & 0.00599677 & 0.0617288 & 0.0676592 & 0.0672147 & 0.110279 & 0.0625858 \\
3.37657 & 0.255194 & 0.0710028 & -0.291611 & 0.0768046 & 0.281297 & 0.0720764 \\
3.53306 & 0.129554 & 0.0816978 & -0.0360767 & 0.088405 & 0.0842794 & 0.0776437 \\
3.70101 & 0.0969659 & 0.0875748 & 0.00417046 & 0.0903624 & 0.0633697 & 0.0845487 \\
3.8554 & 0.207411 & 0.113086 & -0.261385 & 0.117855 & 0.216731 & 0.100849 \\
3.76915 & 0.045445 & 0.153063 & 0.00637958 & 0.178472 & 0.300721 & 0.13973
\end{tabular}

Table A22: Data Points for Fig. 4.14a. 


\begin{tabular}{l|l|l|l|l|l|l}
\hline$-t_{\gamma \rightarrow K^{+}}(\mathrm{GeV})^{2}$ & $c_{1}$ & $\sigma_{\text {stat }}\left(c_{1}\right)$ & $c_{2}$ & $\sigma_{\text {stat }}\left(c_{2}\right)$ & $c_{3}$ & $\sigma_{\text {stat }}\left(c_{3}\right)$ \\
\hline 0.49547 & 0.28433 & 0.0585914 & 0.184116 & 0.0624478 & -0.0446188 & 0.0303098 \\
0.648221 & 0.0973076 & 0.0282316 & 0.270661 & 0.0319757 & 0.0132045 & 0.0188668 \\
0.802995 & -0.124627 & 0.0182284 & 0.205732 & 0.0208933 & 0.030162 & 0.0149145 \\
0.95898 & -0.251617 & 0.015629 & 0.160775 & 0.0176395 & 0.0714335 & 0.0140769 \\
1.11676 & -0.309856 & 0.0154251 & 0.189184 & 0.0173424 & 0.148064 & 0.0146237 \\
1.27671 & -0.345274 & 0.016365 & 0.145831 & 0.0185376 & 0.190649 & 0.0162259 \\
1.43611 & -0.285412 & 0.0174201 & 0.171259 & 0.01972 & 0.175564 & 0.0179277 \\
1.59616 & -0.228209 & 0.0189642 & 0.18136 & 0.0216598 & 0.165495 & 0.0204013 \\
1.75673 & -0.129158 & 0.0201962 & 0.206359 & 0.0231405 & 0.164408 & 0.0223538 \\
1.91928 & -0.0971327 & 0.02148 & 0.112079 & 0.0244562 & 0.10258 & 0.0245511 \\
2.07709 & -0.0108745 & 0.023035 & 0.112747 & 0.0260528 & 0.108022 & 0.0266008 \\
2.23668 & -0.0434146 & 0.0249148 & 0.0796889 & 0.0277461 & 0.0401945 & 0.0285552 \\
2.39922 & -0.128737 & 0.0270155 & 0.0690933 & 0.0300781 & 0.0339674 & 0.0314236 \\
2.55844 & -0.125764 & 0.0288341 & 0.0491639 & 0.0320783 & -0.00676459 & 0.0333901 \\
2.71726 & -0.124145 & 0.0336302 & 0.0851674 & 0.0372811 & -0.029443 & 0.0374037 \\
2.87654 & -0.0708425 & 0.0367648 & 0.0977297 & 0.0410458 & 0.047067 & 0.0400135 \\
3.03625 & -0.254647 & 0.0402342 & 0.0959877 & 0.0443214 & -0.0514927 & 0.0443018 \\
3.19859 & -0.118766 & 0.0460935 & 0.0901625 & 0.050829 & 0.130225 & 0.0480998 \\
3.35559 & -0.190476 & 0.0490882 & 0.155081 & 0.0545357 & 0.129586 & 0.053647 \\
3.51851 & -0.085222 & 0.0600388 & 0.0629213 & 0.0664514 & 0.159687 & 0.0604618 \\
3.67706 & -0.197467 & 0.0698714 & 0.134973 & 0.0769202 & 0.0362505 & 0.0698934 \\
3.83186 & -0.0306248 & 0.078536 & -0.0231074 & 0.0866182 & -0.0480207 & 0.0760237 \\
3.99912 & 0.0122343 & 0.0884553 & 0.0241726 & 0.097479 & 0.0637003 & 0.0871737 \\
4.16004 & 0.183701 & 0.0883843 & -0.0654679 & 0.0932977 & 0.0688181 & 0.0891457 \\
4.322 & -0.00432009 & 0.117562 & -0.0630483 & 0.133225 & 0.185121 & 0.113607
\end{tabular}

Table A23: Data Points for Fig. 4.14b. 
VITA

RAFAEL A. BADUI-CRUZ

$2006-2010$

B.S., Physics

B.S., Mathematics

Florida International University

Miami, Florida

$2010-2016$

Ph.D., Physics

Florida International University

Miami, Florida

\section{SELECTED PUBLICATIONS AND PRESENTATIONS}

Rafael A. Badui, Jason S. Bono, Lei Guo, Brian A. Raue

"The Beam-Helicity Asymmetry for $\gamma p \rightarrow p K^{+} K^{-}$and $\gamma p \rightarrow p \pi^{+} \pi^{-}$" AIP Conf.Proc. $(2016)$

Lei Guo, Rafael A. Badui, Jason S. Bono, Brian A. Raue, "Hyperon Spectroscopy at Hall B, Jefferson Lab” JPS Conf. Proc. (2016)

Rafael A. Badui, Jason S. Bono, Lei Guo, Brian A. Raue, The Beam-Helicity Asymmetry for $\gamma p \rightarrow p K^{+} K^{-}$and $\gamma p \rightarrow p \pi^{+} \pi^{-}$, XVI International Conference on Hadron Spectroscopy

Rafael A. Badui, Jason S. Bono, Lei Guo, Brian A. Raue, Beam-Helicity Asymmetry for $\gamma p \rightarrow p K^{+} K^{-}$, Photonuclear Reactions - 2014 Gordon Research Conference 